ARGONNE NATIONAL LAEORATORY

9700 South. Cass Avenue

Argonne, Illinois 60439

ANL/MCS-TM-- 174

DE 93008116

ANL/MCS-TM- 174

\title{
A Summary of Genomic Data Relating to E. coli \\ Organized by Metabolic Pathways: \\ An Initial Version
}

by

Morgan Price, Muralidhara Raju, and Ronald Taylor*

Mathematics and Computer Science Division

Technical Memorandum No. 174

January 1993

\section{MASTER}

*Division of Computer Research and Technology, National Institutes of Health, Bethesda, MD 20892

This work was supported by the Office of Scientific Computing, U.S. Department of Energy, under Contract W-31-109-Eng-38 


\section{Preface}

We have encoded a summary of the reactions that occur in some of the main metabolic pathways of $E$. coli, and we have integrated these encodings as objects in the GenoBase database being developed by researchers at Argonne National Laboratory in collaboration with groups at the National Institutes of Health and at Ilarvard. This report summarizes the encoded pathways. We have used GenoBase to access objects converted from a number of other existing databases, and we present the integrated form of the data to facilitate detection of inconsistencies and crrors.

In preparing this report, it became clear how useful it was to have access to carefully curated databases that have been made widely available. Most of the data we present in this report was taken directly from entries in these other databases, and so most of this report reflects substantial amounts of effort contributed to the community by a number of curators. We are particularly endebted to the creators of the Swiss Protein Data Bank, The Protein Information Resource, the Enzyme Data Bank, the EcoSeq Database, CompoundKB, and the Database of Metabolic Reactions.

The report is structured by pathway, and each pathway that has been encoded has an entry in the table of contents. For each pathway, the report begins with a summary of the substrates, products, enzymes and cofactors for the pathway as a whole. Then, each reaction in the pathway is described. For each reaction, we show the substrates, products, enzymes, and cofactors.

In addition, for each enzyme, we display a description and activity as listed in the Enzyme Data Bank, followed by the Swiss Protein Data Bank entries that correspond to that enzyme. We consider this information interesting, since it summarizes the set of peptides that relate to the enzyme; when the actual structure of a peptide is known, we underline the identifier. Unfortunately, at this time we have not yet developed a database that allows us to effectively summarize enzymes made up of multiple peptides. That is, the notion of an "abstract peptide" is missing. We plan on adding this category of object to GenoBase, allowing us to relate a number of abstract peptides to a single enzyme, and then to relate each actual peptide to the appropriatc abstract peptide.

For each enzyme, we include separate summary lines for each of the $E$. coli genes that are associated with the enzyme. For each such gene, we list

- the Swiss Protein Data Bank identifier for the peptide corresponding to the gene,

- the gene name,

- where the gene occurs in the genetic map prepared by Barbara Bachmann,

- where the gene occurs on the chromosome in coordinates given by Kenn Rudd, and 
- a set of peptides that have been aligned to the given E.coli peptide and distributed by the PIR.

When data is missing, we indicate the condition with question marks.

We have indexed the occurrences of peptides, enzymes, and genes.

We view this report as just an initial effort. Our main effort will move to integrating data from other genomes, since this summarization from a metabolic perspective seems useful to us. If others wish to extend the set of included pathways or to add information to the existing objects in the database, we will be happy to help with their efforts (by offering access to our data, use of GenoBase, etc.).

We made extensive use of the following reference works:

Bachmann, B. J., Linkage map of Escherichia coli K-I: edition 8, Microbiol. Rev. 54: 130-97 (1990)

James, D. C.; Matthews, G. S., Understanding the Biochemistry of Respiration, Cambridge University Press (1991)

Karp, Peter D., A Kinowledge Base of Chemical Compounds of Intermediary Metabolism, distributed over internet (November 1991)

Kornberg, Arthur, DNA Replication, W. H. Freeman and Co. (1992)

Neidhardt, Frederick C., ed., Escherichia coli and Salmonella typhimurium, American Society for Microbiology (1987)

Ochs, R. S.; Conrow, K., A computerized metabolic map, J. Chem. Inf. Comput. Sci. 31: 132-137 (1991)

Rudd, K. E.; Miller, W.; Ostell, J.; Benson, D. A., Alignment of Escherichia coli K12 DNA sequences to a genomic restriction map, Nucleic Acids Res. 18: 313-21 (1990)

Smith C.A.; Wood E.J. Eneryy in Biological Systems Chapman \& Iall (1991)

Zubay, Geoffrey, Biochemistry, Macmillan Publishing Co. (1988) 


\section{Disclaimer}

This paper is not a final draft. As such, we make no claims as to the accuracy of the information presented. In fact, we go further: it is clear that there are probably numerous minor errors. Our hope in circulating this initial version of the data is that other researchers will both benefit from it and help us arrive at a more accurate version. Please notify us of any errors found, by sending e-mail to price@mcs.anl.gov. 


\section{Contents}

Abstract $\quad$ ix

ATP-Synthesis_1 1

Arginine-Synthesis_1 4

$\begin{array}{ll}\text { Chorismate-Synthesis_1 } & 10\end{array}$

$\begin{array}{ll}\text { CoA-Synthesis } 11 & 16\end{array}$

$\begin{array}{lr}\text { Creatine-Synthesis_1 } & 19\end{array}$

$\begin{array}{ll}\text { Cysteine-Synthesis-1 } & 20\end{array}$

Cysteine-Synthesis $2 \quad 22$

ETC_1 24

ETC_2 31

FAD-Synthesis_1 39

Fat-Degradation_1 40

GTP-Synthesis 1

$\begin{array}{ll}\text { Gluconeogenesis_ } 1 & 47\end{array}$

$\begin{array}{ll}\text { Glutamate-Synthesis_1 } & 57\end{array}$

$\begin{array}{ll}\text { Glycolysis } 1 & 60\end{array}$

Histidine-Synthesis 1 68

$\begin{array}{ll}\text { Homoserine-Synthesis } 1 & 74\end{array}$

$\begin{array}{ll}\text { IMP-Synthesis_1 } & 77\end{array}$

Isoleucine-Synthesis_1

Lysine-Synthesis_1

$\begin{array}{ll}\text { Methionine-Synthesis_1 } & 97\end{array}$

$\begin{array}{ll}\text { Methionine-Synthesis_2 } & 100\end{array}$

$\begin{array}{ll}\text { NAD-Synthesis } 1 & 103\end{array}$ 
NAD-Synthesis 2

Palmitoyl-ACP-Synthesis_1 106

$\begin{array}{ll}\text { Pentose-Shunt } 1 & 110\end{array}$

Phenylalanine-Synthesis 1

Proline-Synthesis 1118

$\begin{array}{ll}\text { Purixe-Synthesis_1 } & 121\end{array}$

Pyrimidine-Synthesis 1

$\begin{array}{ll}\text { Serine_1 } & 139\end{array}$

$\begin{array}{ll}\text { TCA_1 } & 141\end{array}$

THF-Synthesis 1

Threonine-Synthesis_1 152

Tryptophan-Synthesis_1 154

$\begin{array}{ll}\text { Tyrosine-Synthesis } & 159\end{array}$

Valine-Synthesis_1 162

$\begin{array}{ll}\text { Valine-Synthesis_2 } & 167\end{array}$

Index 182

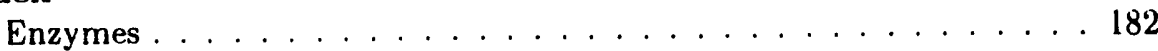

Genes . . . . . . . . . . . . . . . . . . 193

Peptides . . . . . . . . . . . . . . . . . 198

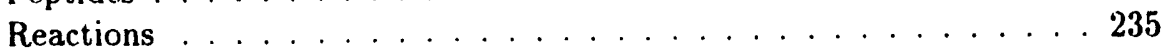




\title{
A Summary of Genomic Data Relating to E. coli Organized by Metabolic Pathways: An Initial Version
}

\author{
by \\ Morgan Price \\ Muralidhara Raju \\ Ronald Taylor
}

\begin{abstract}
This report summarizes the reactions that occur in some of the principal metabolic pathways of $E$. coli. These pathways have been encoded as objects in GenoBase, an integrated database under development at Argonne National Laboratory in collaboration with researchers at the National Institutes of Health and at Harvard University. The report lists the substrates, products, enzymes, and cofactors for each pathway as a whole, followed by a detailed description of each reaction in the pathway. In addition, for each enzyme, the report displays a description and activity as listed in the Enzyme Data Bank, frliowed by the corresponding Swiss Protein Data Bank entries. Separate summary ines are included for each of the $E$. coli genes associated with each enzyme.
\end{abstract}




\section{Summary of the Pathway: ATP-Synthesis_1}

\begin{tabular}{|rcl|}
\hline & $\begin{array}{l}2.7 .4 .3 \\
2.7 .4 .6\end{array}$ & \\
ATP & $\mathbf{6 . 3 . 2 . 2}$ & 2.00 ADP \\
GTP & ATP & Fumarate \\
IMP & $\rightleftharpoons$ & GDP \\
L-Aspartate & & $P_{i}$ \\
\hline
\end{tabular}

Reaction: IMP_Adenine_1

\begin{tabular}{|c|c|c|}
\hline $\begin{array}{l}\text { GTP } \\
\text { IMP }\end{array}$ & $\stackrel{6.3 .4 .4}{\rightleftharpoons}$ & $\begin{array}{l}\text { Adenylosuccinate } \\
\text { GDP }\end{array}$ \\
\hline L-Aspartate & & $P_{i}$ \\
\hline
\end{tabular}

\subsubsection{4: Adenylosuccinate Synthase}

P21900 P12283

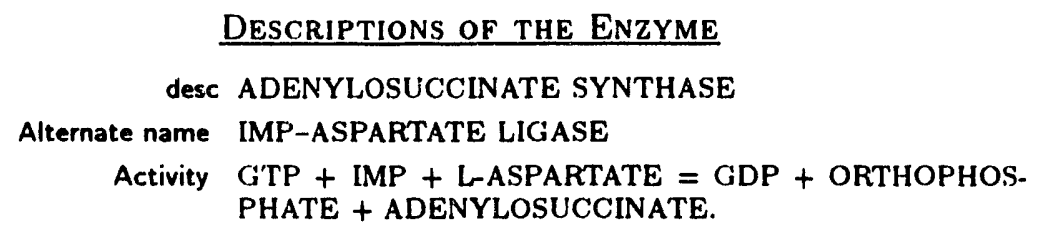

DESCRIPTIONS OF THE ENZYME

desc ADENYLOSUCCINATE SYNTHASE

Alternate name IMP-ASPARTATE LIGASE

Activity G'TP + IMP + L-ASPARTATE = GDP + ORTHOPHOSPHATE + ADENYLOSUCCINATE.

Pl2283 purA 95.004435934 to 4437232

\section{DEscriptions OF THE PEPTIDE}

desc ADENYLOSUCCINATE SYNTHETASE (EC 6.3.4.4) (IMPASPARTATE LIGASE)

E.coli gene PURA

\section{DESCRIPTIONS OF THE CDS}

desc adenylosuccinate synthetase

Reaction: Adenylosucc $\_$Adenine_1

Adenylosuccinate $\stackrel{4.3 .2 .2}{=}$ AMP

4.3.2.2: Adenylosuccinate Lyase 


\section{DESCRIPTIONS OF THE ENZYME}

desc ADENYLOSUCCINATE LYASE

Alternate name ADENYLOSUCCINASE

Activity ADENYLOSUCCINATE = FUMARATE + AMP.

Pep??? purB 25.42 Loc???

\section{DESCRIPTIONS OF THE CDS}

desc adenylosuccinate lyase

Reaction: AMP_Adenine_1

$$
\underset{A T P}{\operatorname{AMP}} \stackrel{2.7 .4 .3}{=} 2.00 * A D P
$$

2.7.4.3: Ader:ylaie K'inase

$\begin{array}{lllll}\text { P00570 } & \text { P } 05081 & \text { P00568 } & \text { P00571 } & \text { P00569 } \\ \text { P07170 } & P 08166 & \text { P16304 } & \text { P12115 } & \text { P05082 } \\ \text { P24323 } & \text { P } 10251 & \text { P10772 } & & \end{array}$

DESCRIPTIONS OF THE ENZYME

desc ADENYLATE KINASE

Alternate name MYOKINASE

Activity ATP + AMP = ADP + ADP.

P05082 adk 10.89 Loc???

Descriptions OF THE PEPTIDE

desc ADENYLATE KINASE (EC 2.7.4.3) (ATP-AMP TRANSPHOSPHORYLASE)

E.coli gene ADK

\section{DESCRIPTIONS OF THE CDS}

desc adenylate kinase activity; pleiotropic effects on glycerol-3phosphate acyltranserase activity 


\section{Reaction: ADP_Adenine_1}

$$
\begin{array}{lll}
\text { ADP } & 2.7 .4 .6 & \text { ADP } \\
\text { ATP } & & \text { ATP }
\end{array}
$$

\subsubsection{6: Nucleoside-Diphosphate Kinase}

DESCRIPTIONS OF THE ENZYME

desc NUCLEOSIDE-DIPHOSPHATE KINASE

Alternate name NUCLEOSIDE 5'-DIPHOSPHATE PHOSPHOTRANSFERASE

Alternate name NDK

Activity ATP + NUCLEOSIDE DIPHOSPHATE = ADP + NUCLE OSIDE TRIPHOSPHATE.

Pep??? cds??? Pos??? Loc??? 


\section{Summary of the Pathway: Arginine-Synthesis_1}

\begin{tabular}{|c|c|c|}
\hline $\begin{array}{r}\text { Acetyl-(:oA } \\
2.00 * A T P \\
\text { (arbamoyl-Phosphate } \\
2.00 * \text { Gilutamate } \\
\text { L- Aspartate } \\
\mathrm{NADPH}_{2} \\
\mathrm{H}_{2} \mathrm{O}\end{array}$ & $\begin{array}{c}1.2 .1 .38 \\
2.1 .3 .3 \\
2.3 .1 .1 \\
2.6 .1 .11 \\
2.7 .2 .8 \\
3.3 .1 .16 \\
4.3 .2 .1 \\
5.3 .1 .5 \\
\text { Pyridoxal Phosphate }\end{array}$ & $\begin{array}{l}\text { 2-Ketoglutarate } \\
\text { Acetate } \\
\text { ADP } \\
\text { AMP } \\
\text { Arginine } \\
\text { CoA } \\
\text { Fumarate } \\
\text { NADP } \\
2.00 \text { P }_{\mathbf{i}} \\
\text { PPi }\end{array}$ \\
\hline
\end{tabular}

Reaction: GLT_3

$$
\begin{gathered}
\text { Acetyl-CoA } \stackrel{2.3 .1 .1}{=} \text { f.ceiyl-Glutamate } \\
\text { Glutamate }
\end{gathered}
$$

2.3.1.1: Amino-Acid Acetyltransferase

P08205 P22567

DESCRIPTIONS OF THE ENZYME

desc AMINO-ACID ACETYLTRANSFERASE

Activity ACETYL-COA + L-GLUTAMATE $=$ COA + N-AC:ETYL L-GLUTAMATE.

P08205 argA 60.522967443 to 2968774

\section{Descriptions of THE PEPTIDE}

desc AMINO-ACID ACETYLTRANSFERASE (N-ACETYLGLUTAMATE SYNTHASE) (EC 2.3.1.1).

E.coli gene ARGA

DESCRIPTIONS OF THE CDS

desc amino acid acetyltransferase; $\mathrm{N}$-acetylglucosanine synthase 
Reaction: Acetyl-GLU_1

$$
\begin{aligned}
& \text { Acetyl-Glutamate } \stackrel{2.7 .2 .8}{\rightleftharpoons} \mathrm{ADr} \\
& \text { ATP } N \text {-Acetyl-r-Glutamyl-P }
\end{aligned}
$$

2.7.2.8: Acetylglutamale Kinase

P11445

$$
\begin{aligned}
& \text { DESCRIPTIONS OF THE ENZYME } \\
& \text { desc ACETYLGLUTAMATE KINASE } \\
& \text { Activity ATP + N-ACETYL-L-GLUTAMATE = ADP + N-ACETYL } \\
& \text { L-GLUTAMATE 5-PHOSPHATE. }
\end{aligned}
$$

P11445 argB 89.494185120 to 4185896

\section{DESCRIPTIONS OF THE PEPTIDE}

des. ACETYLGLUTAMATE KINASE (EC 2.7.2.8) (NAG KINASE)

E.coli gene ARGB

DESCRIPTIONS OF THE CDS

desc acetylglutamate kinase

\section{Reaction: N-Acetyl-Glutamyl-P_1}

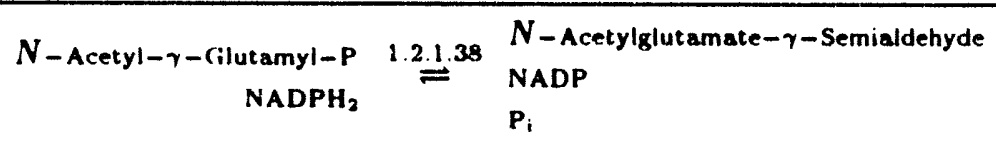

1.2.1.38: N-Acetyl-y-Glatamyl-Phosphate Reductase

P23715 P11446

DESCRIP'TIONS OF TAE ENZYME

desc N-ACETYLGAMMA-GLUTAMYL-PHOSPHATE REDUCTASE

Alternate name N-ACETYL-GLUTAMATE SEMIALDEHYDE DEHYDROGENASE

Alteriate name NAGSA DEHYDROGENASE 


$$
\begin{array}{ll}
\text { Activity } & \text { N-ACETYL-L-GLUTAMATE 5-SEMIALDEHYDE + NADP(+) } \\
& + \text { ORTHOPHOSPHATE = N-ACETYL-5-GLUTAMYL PHOS- } \\
& \text { PHATE + NADPH. }
\end{array}
$$

Pl1446 argC 89.494184108 to 4185112

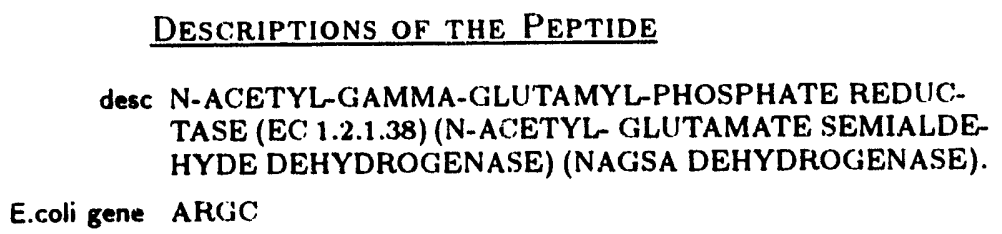

Reaction: N-AcetylGlutanate_Semialdehyde 1

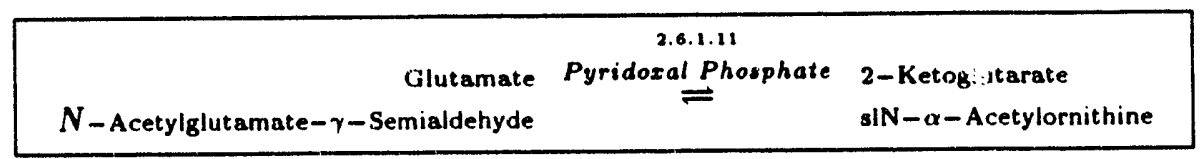

2.6.1.11: Acetylornithine Aminoti unsferase

P18335 P18544

DESCRIPTIONS OF THE ENZYME

desc AC:ETYLORNITHINE AMINOTRANSFERASE

Activity N2-ACETYL-LORNITHINE + 2-OXOGLUTARATE $=$ NACETYL-L-GLUTAMATE 5-SEMIALDEHYDE + L-GLUTAMATE.

P18x35 argD 73.803509746 from 3510966 complement

Descriptions of the Peptide

desc ACETYLORNITHINE AMINOTRANSFERASE (EC 2.6.1.11) (ACOAT)

E.coli gene ARGD

DESCRIPTIONS OF THE CDS 
desc acetylonithine delta-aminotransferase

Reartion: N-Alpha-Acetyl-Ornithine_1

$$
\begin{aligned}
& \text { sIN- } \alpha \text {-Acetylornithine 3.5.1.16 Acetate } \\
& \mathrm{H}_{2} \mathrm{O} \rightleftharpoons \text { L-Ornithine }
\end{aligned}
$$

3.5.1.16: Acetylornithine Deacetylase

\section{DESCRIPTIONS OF THE ENZYME}

desc ACETYLORNITHINE DEACETYLASE

Alternate name ACETYLORNITHINASE

Activity N2-ACETYL-L-ORNITHINE + H(2)O = ACETATE + LORNITHINE.

Pep??? argE 89.494183908 from 4183954 complement

DESCRIPTIONS OF THE CDS

desc acetylornithine deacetylase

Reaction: L-Oruithine_1

$$
\begin{aligned}
\text { Carbamoyl-Phosphate } & \stackrel{2.1 .3 .3}{=} \text { Citrulline } \\
\text { ᄂ-Ornithir. } & \mathrm{P}_{\mathrm{i}}
\end{aligned}
$$

2.1.3.3: Ornithine Carbamoyltransferase

$\begin{array}{lllll}\text { P04391 } & \text { P18186 } & \text { P06960 } & \text { P11724 } & \text { P11726 } \\ \text { P21302 } & \text { P08308 } & \text { P11727 } & \text { P23752 } & \text { P16964 } \\ \text { P11066 } & \text { P11803 } & \text { P00480 } & \text { P11725 } & \text { P14995 } \\ \text { P00481 } & \text { P05150 } & & & \end{array}$

DESCRIPTIONS OF THE ENZYME

desc ORNITHINE CARBAMOYLTRANSFERASE

Alternate name ORNITHINE TRANSCARBAMYLASE

Alternate name CITRULLINE PHOSPHORYLASE

Alternate name OTC

Activity CARBAMOYL PHOSPHATE + L-ORNITHINE $=$ ORTHOPHOSPHATE + L-CITRULLINE. 
P04391 argl 96.574506986 from 4507990 complement

\section{DEscriptions of THE PEPTIDE}

desc ORNITHINE CARBAMOYLTRANSFERASE (EC: 2.1.3.3), CHAIN I (OTCASE-1)

E.coli gene ARGI

\section{DESCRIPTIONS OF THE CDS}

desc Omithine carbamoyltransferase (duplicate gene)

P06960 argF 6.48297009 from 298013 complement

DESCRIPTIONS OF THE PEPTIDE

desc ORNITHINE CARBAMOYLTRANSFERASE (EC 2.1.3.3), CHAIN F (OTCASE-2)

E.coli gene ARGF

DESCRIPTIONS OF THE CDS

desc ornithine carbamoyltransferase (duplicate gene)

\section{Reaction: Citrulline_1}

\begin{tabular}{rll|} 
ATP & 6.3.4.5 & AMP \\
Citrulline & Argininosuccinate \\
L-Aspartate & & $\mathrm{PP}_{\mathrm{i}}$ \\
\hline
\end{tabular}

\subsubsection{5: Argininosuccinate Synthase}

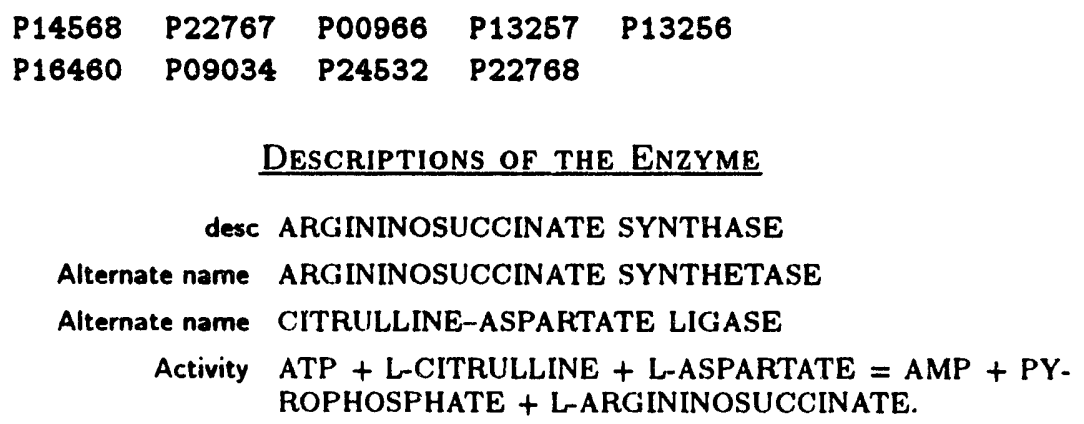

DESCRIPTIONS OF THE ENZYME

desc ARGININOSUCCINATE SYNTHASE

Alternate name ARGININOSUCCINATE SYNTHETASE

Alternate name CITRULLINE-ASPARTATE LIGASE

Activity ATP + L-CITRULLINE + L-ASPARTATE = AMP + PYROPHOSPHATE + L-ARGININOSUCCINATE. 
P22767 arg(i 68.993337708 to 3339051

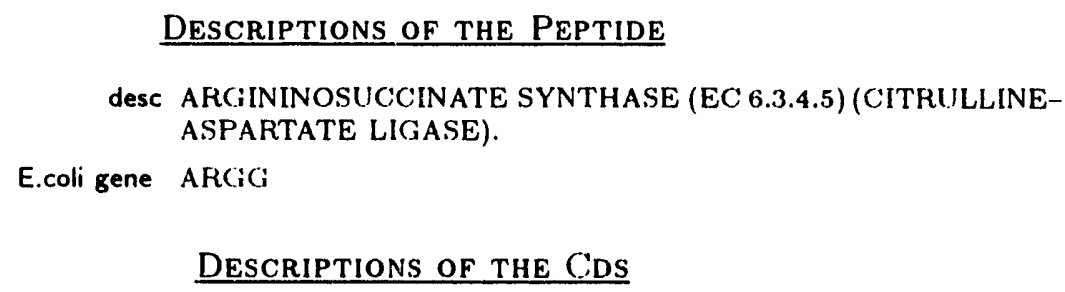

DESCRIPTIONS OF THE CDS

desc argininosuccinate synthetase

Reaction: Argininosucc_1

Argininosuccinate $\stackrel{4.3 .2 .1}{\rightleftharpoons}$ Arginine

4.3.2.1: Argininosuccinate Lyase

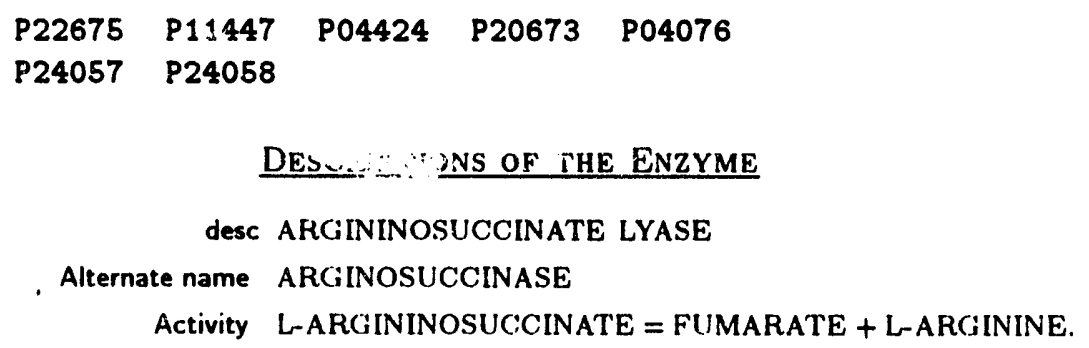

P11447 argH 89.504185957 to 4186108

\section{DESCRIPTIONS OF THE PEPTIDE}

desC ARGININOSUCCINATE LYASE (EC 4.3.2.1) (ARGINOSUCCINASE) (FRAGMENT)

E.coli gene ARGH

\section{DESCRIPTIONS OF THE CDS}

desc argininosuccinate lyase 


\section{Summary of the Pathway: Chorismate-Synthesis_1}

\begin{tabular}{|c|c|c|}
\hline $\begin{array}{r}\text { ATP } \\
\text { Erythrose-4-P } \\
\text { NADPH }_{2} \\
\text { 2.00* Phosphoenolpyruvate } \\
\mathrm{H}^{+}\end{array}$ & $\begin{array}{l}1.1 .1 .23 \\
2.3 .1 .19 \\
2.7 .1 .71 \\
1.1 .2 .15 \\
4.2 .1 .10 \\
1.6 .1 .3 \\
1.6 .1 .4 \\
\mathrm{NAD} \\
\mathrm{H}_{2} \mathrm{O}\end{array}$ & $\begin{array}{l}\text { ADP } \\
\text { Chorismate } \\
\text { NADP } \\
4.00 * P_{i}\end{array}$ \\
\hline
\end{tabular}

Reaction: PEP_1

$$
\begin{array}{rrl}
\text { Erythrose-4-P } & \text { 4.1.2.15 } & 3-\text { Deoxy-D-Arabino-Heptulosonate-7-P } \\
\text { Phosphoenolpyruvate } & \stackrel{P_{i}}{=} \mathrm{H}_{2} \mathrm{O} &
\end{array}
$$

4.1.2.15: Phospho-2-Dehydro-3-Deoxyheptonate Aldolase

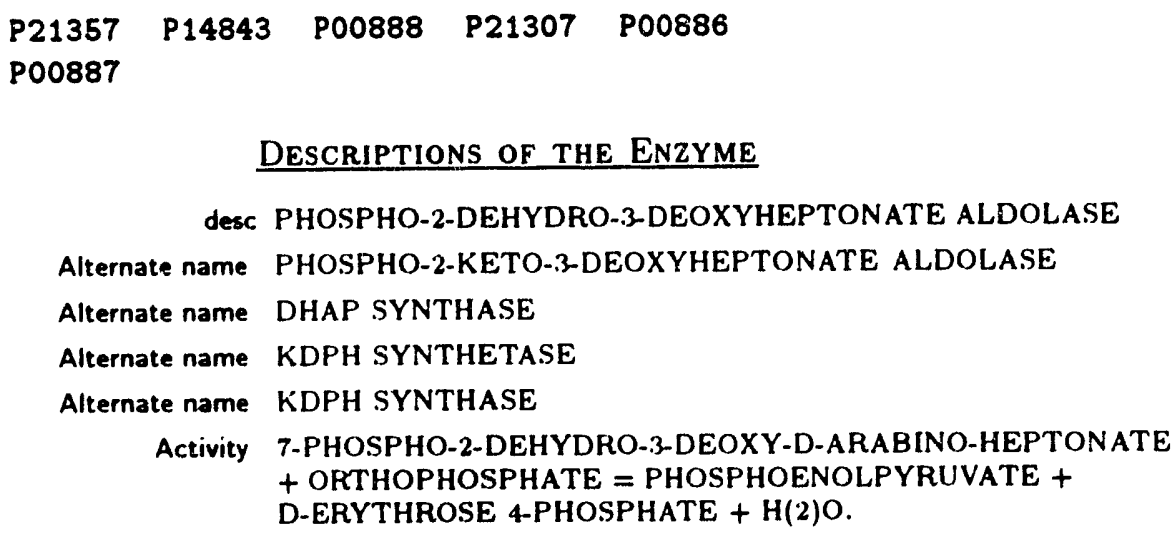

DESCRIPTIONS OF THE ENZYME

desc PHOSPHO-2-DEHYDRO-3-DEOXYHEPTONATE ALDOLASE

Alternate name PHOSPHO-2-KETO-3-DEOXYHEPTONATE ALDOLASE

Alternate name DHAP SYNTHASE

Alternate name KDPH SYNTHETASE

Alternate name KDPH SYNTHASE

Activity 7-PHOSPHO-2-DEHYDRO-3-DEOXY-D-ARABINO-HEPTONATE + ORTHOPHOSPHATE $=$ PHOSPHOENOLPYRUVATE + D-ERYTHROSE 4-PHOSPHATE + H(2)O.

P00886 aroG 16.80794933 to 795985

\section{Descriptions of the PEPTIDE}

desc PHOSPHO-2-DEHYDRO-3-DEOXYHEPTONATE ALDOLASE, PHE-SENSITIVE (EC 4.1.2.15) (PHOSPHO-2-KETO-3-DEOXYHEPTONATE ALDOLASE) (DAHP SYNTHETASE).

E.coli gene AROG

DESCRIPTIONS OF THE CDS 
desc DAHP synthetase (phenylalanine repressible)

P00887 aroH 37.201796839 to 1797885

Descriptions of the Peptide

desc PHOSPHO-2-DEHYDRO-3-DEOXYHEPTONATE ALDOLASE,

TRP-SENSITIVE (EC 4.1.2.15) (PHOSPHO-2-KETO-3-DEOXYHEPTONATE ALDOLASE) (DAHP SYNTHETASE).

E.coli gene AROH

DESCRIPTIONS OF THE CDS

desc DAHP synthetase (tryptophan repressible)

P00888 aroF 56.642753198 from 2754268 complement

\section{DESCRIPTIONS OF THE PEPTIDE}

desc PHOSPHO-2-DEHYDRO-3-DEOXYHEPTONATE ALDOLASE, TYR-SENSITIVE (EC 4.1.2.15) (PHOSPHO-2-KETO-3-DEOXYHEPTONATE ALDOLASE) (DAHP SYNTHETASE).

E.coli gene AROF

DESCRIPTIONS OF THE CDS

desc DAHP synthetase (tyrosine repressible)

\section{Reaction: ?-denxy-D-Arabino-Heptulosonate-7-P 1}

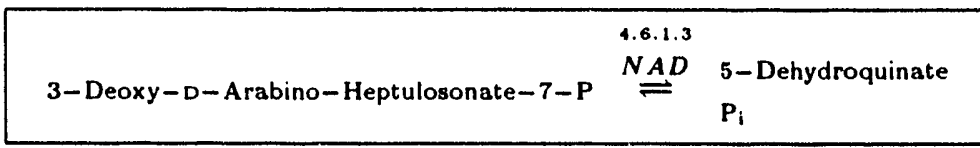

4.6.1.3: 3-Dehydroquinate Synthase

P07547 P08566 P07639

DESCRIPTIONS OF THE ENZYME

desc 3-DEHYDROQUINATE SYNTHASE

Activity 7-PHOSPHO-3-DEOXY-ARABINO-HEPTULOSONATE $=3$ DEHYDROQUINATE + ORTHOPHOSPHATE. 
P07639 aroB 74.603538821 from 3539909 complement

DESCRIPTIONS OF THE PEPTIDE

desc 3-DEHYDROQUINATE SYNTHASE (EC: 4.6.1.3)

E.coli gene AROB

DESCRIPTIONS OF THE CDS

desc Dehydroquinate synthase

Reaction: 5-dehydro-Quinate 1

$$
\text { 5-Dehydroquinate } \stackrel{4.2 .1 .10}{\rightleftharpoons} \begin{aligned}
& 5-\text { Dehydro-Shikimate } \\
& \mathrm{H}_{2} \mathrm{O}
\end{aligned}
$$

4.2.1.10: 3-Dehydroquinate. Dehydralase

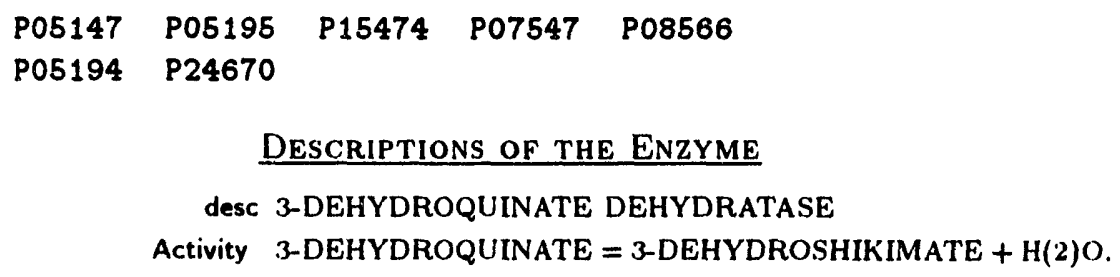

DESCRIPTIONS OF THE ENZYME

desc 3-DEHYDROQUINATE DEHYDRATASE

Activity 3-DEHYDROQUINATE = 3-DEHYDROSHIKIMATE + H(2)O.

P05194 aroD 37.141784352 to 1785074

Descriptions of THE PEPTIDE

desc 3-DEHYDROQUINATE DEHYDRATASE (EC 4.2.1.10) (3DEHYDROQUINASE)

E.coli gene AROD

DESCRIPTIONS OF THE CDS

desc 3-Dehydroquinate dehydratase

Reaction: 5-dehydro-Shikimate 1

5-Dehydro-Shikimate 1.1.1.25 NADP $\mathrm{NADPH}_{2}$ Shikimate

1.1.1.25: Shikimate Dehydrogenase

P07547 P08566 P15770 


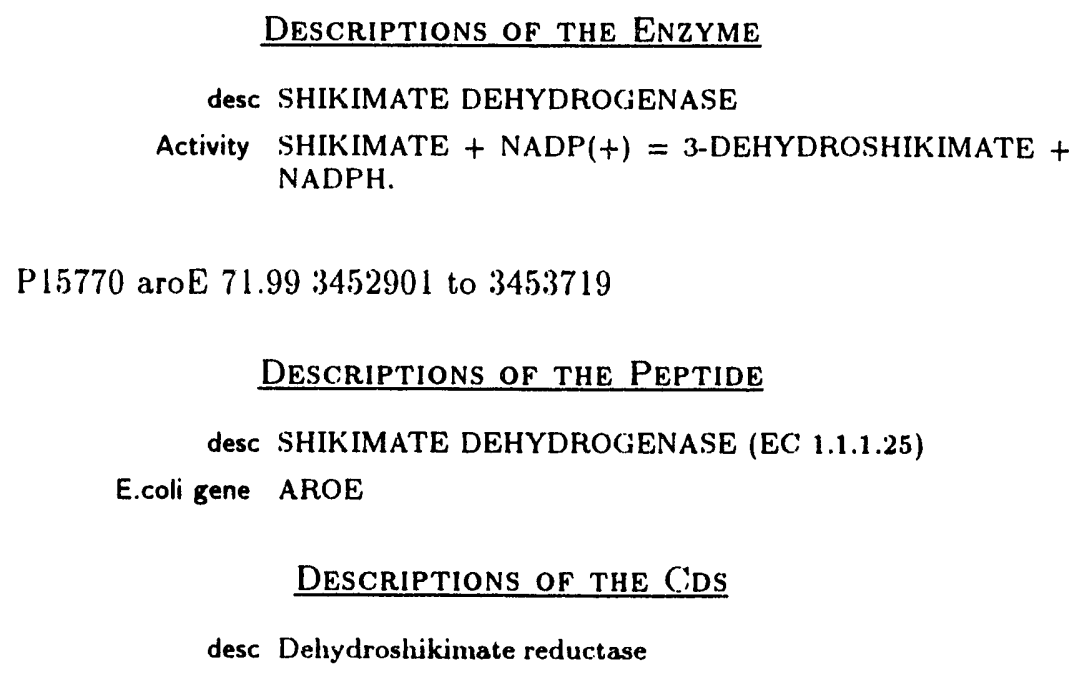

Reaction: Shikimate 1

\begin{tabular}{rll|}
\hline ATP & 2.7.1.71 & ADP \\
$\mathrm{H}^{+}$ & $\rightleftharpoons$ & Shikimate-5-P \\
Shikimate & & \\
\hline
\end{tabular}

\subsubsection{1: Shikimate Kinase

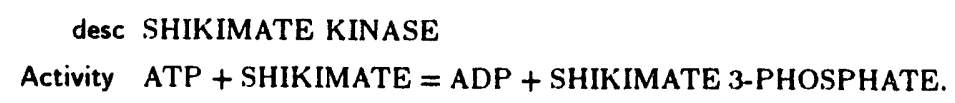

\section{DESCRIPTIONS OF THE CDS}

desc Shikimate kinase II 
Reaction: Shikimate-5P_1

Phosphoenolpyruvate $\stackrel{2.5 .1 .19}{=} 3$-Enolpyruvyl-Shikimate-5-Phosphate

Shikimate-5-P $P_{i}$

2.5.1.19: 3-Phosphoshikimate 1-Carboxyvinyltransferase

$\begin{array}{lllll}\text { P07547 } & \text { P23981 } & \text { P08566 } & \text { P23281 } & \text { P05466 } \\ \text { P20691 } & \text { P12421 } & \text { P17688 } & \text { P07638 } & \text { P24497 } \\ \text { P10748 } & \text { P22487 } & \text { P11043 } & \text { P22299 } & \text { P } 19786 \\ \text { P07637 } & \text { P19688 } & & & \end{array}$

DESCRIPTIONS OF THE ENZYME

desc 3-PHOSPHOSHIKIMATE 1-CARBOXYVINYLTRANSFERASE

Alternate name 5-ENOLPYRUVYLSHIKIMATE-3-PHOSPHATE SYNTHASE

Alternate name EPSP SYNTHASE

Alternate name 3-ENOL-PYRUVOYLSHIKIMATE-5-PHOSPHATE SYNTHASE

Activity PHOSPHOENOLPYRUVATE + 3-PHOSPHOSHIKIMATE

= ORTHOPHOSPHATE + O5- (1-CARBOXYVINYL)-3-PHOSPHOSHIKIMATE.

P076.38 aroA 20.31967114 to 968397

\begin{tabular}{|llll} 
P07637 & P12421 & P19688 & P22299
\end{tabular}

DESCRIPTIONS OF THE PEPTIDE

desc 3-PHOSPHOSHIKIMATE 1-CARBOXYVINYLTRANSFERASE

(EC 2.5.1.19) (5- ENOLPYRUVYLSHIKIMATE-3-PHOSPHATE SYNTHASE) (EPSP SYNTHASE).

E.coli gene AROA

\section{DESCRIPTIONS OF THE CDS}

desc 3-enol-pyruvoylshikimate-5-phosphate synthase

Reaction: 3-Enolpyruvyl-Shikimate-5P 1

3-Enolpyruvyl-Shikimate-5-Phosphate

4.6.1.4 Chorismate

$P_{i}$

4.6.1.4: Chorismate Synthase

P12008 P16280 P23353 


\author{
DESCRIPTIONS OF THE ENZYME \\ desc CHORISMATE SYNTHASE \\ Activity O5-(1-CARBOXYVINYL)-3-PHOSPHOSHIKIMATE = CHO- \\ RISMATE + ORTHOPHOSPHATE. \\ P12008 aroC 50.492464517 from 2465587 complement \\ Descriptions of the Peptide \\ desc CHORISMATE SYNTHASE (EC 4.6.1.4) \\ E.coli gene AROC

\section{DESCRIPTIONS OF THE CDS} \\ desc Chorismate synthase
}


Summary of the Pathway: CoA-Synthesis_1

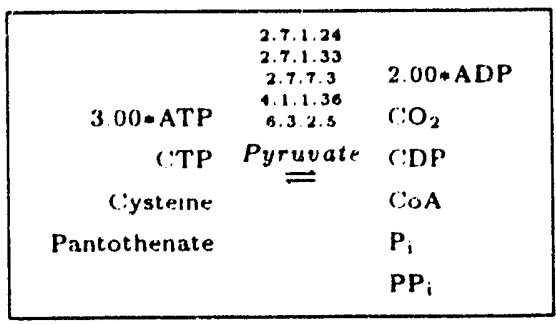

Reaction: Pantothenate_CoA_1

$$
\begin{aligned}
\text { ATP } 2.7 .1 .33 & 4^{\prime}-P \text {-Pantothenate } \\
\text { Pantothenate } & \text { ADP }
\end{aligned}
$$

2.7.1.33: Pantolhenate Kinase

P15044

DESCRIPTIONS OF THE ENZYME

desc PANTOTHENATE KINASE

Activity ATP + PANTOTHENATE $=$ ADP + D-4'-PHOSPHOPANTOTHENATE.

P15044 rts 89.794203293 from 4204303 complement

Descriptions of the Peptide

desc PANTOTHENATE KINASE (EC 2.7.1.33) (RTS PROTEIN)

E.coli gene COAA

\section{DESCRIPTIONS OF THE CDS}

desc uncharacterized growth defect

Reaction: 4'-p-pantothenate_CoA_1

\begin{tabular}{|c|c|c|}
\hline $\begin{array}{r}4^{\prime}-P-P a n t o t h e n a t e \\
C T P\end{array}$ & $\stackrel{6.3 .2 .5}{=}$ & $\begin{array}{l}4^{\prime}-P-N-P a n t o t h e n o y l c y s t e i n e \\
\text { CDP }\end{array}$ \\
\hline Cysteine & & $P_{i}$ \\
\hline
\end{tabular}

6.3.2.5: Phosphopantolhenate-Cysteine Ligase 


\section{DESCRIPTIONS OF THE ENZYME}

desc PHOSPHOPANTOTHENATE-CYSTEINE LIGASE

Alternate name PHOSPHOPANTOTHENOYLCYSTEINE SYNTHETASE

Activity CTP + (R)-4'-PHOSPHOPANTOTHENATE + L-CYYSTEINE $=$ UNIDENTIFIED PRODUCTS OF CTP BREAKDOWN + (R)-4"-PHOSPHOPANTOTHENOYL-L-CYSTEINE.

Pep??? cds??? Pos??? Loc???

Reaction: 4'-p-n-pantothenoylcysteine_CoA_1

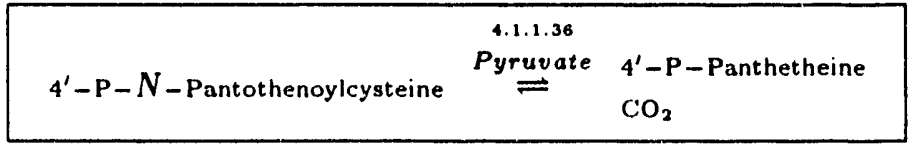

4.1.1.36: Phosphopantothenoylcysteine Decarboxylase

\section{DESCRIPTIONS OF THE ENZYME}

desc PHOSPHOPANTOTHENOYLCYSTEINE DECARBOXYLASE

Activity $\mathrm{N}-((\mathrm{R})-4-\mathrm{PHOSPHOPANTOTHENOYL)-L-CYSTEINE}=\mathrm{PAN}$ TOTHEINE 4'-PHOSPHATE + CO(2).

Pep??? cds??? Pos??? Loc???

Reaction: 4'-p-panthetheine_CoA_1

$$
\begin{aligned}
& 4^{\prime}-\mathrm{P}-\mathrm{Panthetheine} \stackrel{2.7 .7 .3}{\rightleftharpoons} \text { Dephospho-CoA } \\
& \text { ATP } \mathrm{PP}_{\mathrm{i}}
\end{aligned}
$$

\subsubsection{3: Pantetheine-Phosphate Adenylyltransferase}

DESCRIPTIONS OF THE ENZYME

desc PANTETHEINE-PHOSPHATE ADENYLYLTRANSFERASE

Alternate name DEPHOSPHO-COA PYROPHOSPHORYLASE

Activity ATP + PANTETHEINE 4'-PHOSPHATE = PYROPHOSPHATE + DEPHOSPHO-COA. 
Pep??? cds???? Pos??? Loc???

\section{Reaction: Dephospho-CoA_CoA_1}

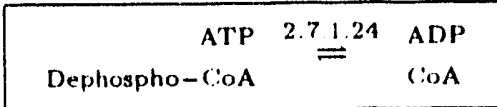

\subsubsection{4: Dephospho-CoA Kinase}

\section{DEsCRIPTIONS OF THE ENZYME}

desc DEPHOSPHO-COA KINASE

Activity $\mathrm{ATP}+\mathrm{DEPHOSPHO}-\mathrm{COA}=\mathrm{ADP}+\mathrm{COA}$.

Pep??? cds??? Pos??? Loc??? 


\section{Summary of the Pathway: Creatine-Synthesis_1}

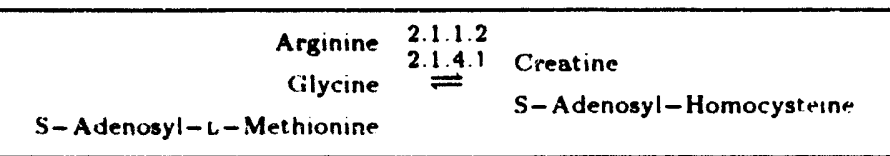

Reaction: ARG_Creatine $\lambda$

$$
\begin{aligned}
& \text { Arginine } 2.1 .1 \text { Guanidino-Acetate } \\
& \text { Glycine } \\
& \text { L-Ornithine }
\end{aligned}
$$

2.1.4.1: Glycine Amidinotransferase

$$
\begin{aligned}
& \frac{\text { DESCRIPTIONS OF THE ENZYME }}{\cdot} \\
& \text { desC GLYCINE AMIDINOTRANSFERASE } \\
& \text { Activity L-ARGININE + GLYCINE = L-ORNITHINE + GUANIDOAC- } \\
& \text { ETATE. }
\end{aligned}
$$

Pep??? cds??? Pos??? Loc???

\section{Reaction: L-Ornithine_Creatine_1}

Guanidino-Acetate
L-Ornithine $\stackrel{2.1 .1 .2}{=} \begin{aligned} & \text { Creatine } \\ & \text { S-Adenosyl-Homocysteine } \\ & \text { S-Adenowyl-L-Methionine }\end{aligned}$

2.1.1.2: Guanidinoacetate Methyltransferase

DESCRIPTIONS OF THE ENZYME

desc GUANIDINOACETATE METHYLTRANSFERASE

Activity S-ADENOSYL-L-METHIONINE + GUANIDOACETATE = S-ADENOSYLLLHOMOCYSTEINE + CREATINE.

Pep??? cds??? Pos??? Loc??? 


\section{Summary of the Pathway: Cysteine-Synthesis-1}

$$
\begin{array}{rll}
\text { Acetyl-CoA } & 2.3 .1 .30 & \text { Acetate } \\
\text { Serine } & \stackrel{2.99 .8}{\text { CoA }} \\
\text { Sultide } & & \begin{array}{l}
\text { Cysterne } \\
\mathrm{H}^{+}
\end{array}
\end{array}
$$

Reaction: SER_CYS_1

$$
\begin{aligned}
& \text { Acetyl-CoA } 2.3 .1 .30 \\
& \text { Serıne } \text { CoA } \\
& \text { O-Acetyl-し-Serine }
\end{aligned}
$$

2.3.1.30: Serine Acetyltransferase

P05796 P23145

DESCRIPTIONS OF THE ENZYME

desc SERINE ACETYLTRANSFERASE

Activity ACETYL-COA + L-SERINE = COA + O-ACETYL-L-SERINE.

P05796 cysE 80.943805423 from 3806244 complement

\section{DESCRIPTIONS OF THE PEPTIDE}

desc SERINE ACETYLTRANSFERASE (EC 2.3.1.30) (SAT)

E.coli gene CYSE

DESCRIPTIONS OF THE CDS

desc Serine acetyltransferage

Reaction: O-Acetyl-L-Serine_CYS_1

$$
\begin{aligned}
& \text { O-Acetyl-L-Serine 4.2.99.8 Acetate } \\
& \text { Sulfide } \rightleftharpoons \quad \mathrm{Cystei}
\end{aligned}
$$

4.2.99.8: O-Acetylserine (Thiol)-Lyase

P11096 P12674 P16703 P06106 


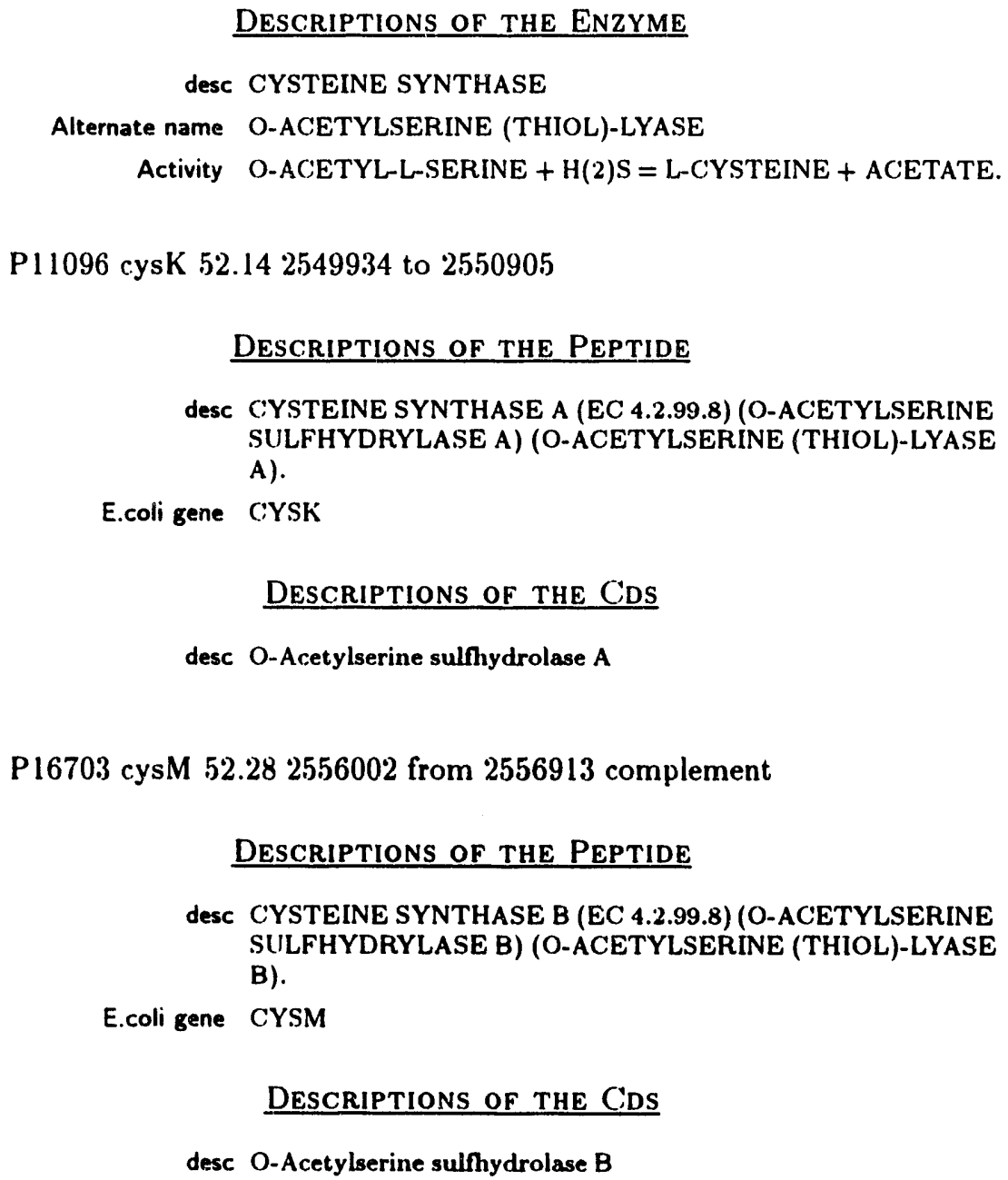

P16703 cysM 52.282556002 from 2556913 complement

\section{Descriptions of the Peptide}

desc CYSTEINE SYNTHASE B (EC 4.2.99.8) (O-ACETYLSERINE SULFHYDRYLASE B) (O-ACETYLSERINE (THIOL)-LYASE B).

E.coli gene CYSM

DESCRIPTIONS OF THE CDS

desc O-Acetylserine sulfhydrolase $B$ 


\section{Summary of the Pathway: Cysteine-Synthesis_2}

\begin{tabular}{|c|c|c|}
\hline $\begin{array}{r}\text { ATP } \\
\text { L-Homocysteine } \\
\text { ᄂ-Methionine } \\
\text { Serine } \\
\mathrm{H}_{2} \mathrm{O}\end{array}$ & $\begin{array}{c}2.5 .1 .6 \\
4.2 .1 .22 \\
4.4 .1 .1 \\
\text { Pyridoxal Phosphate } \\
\mathrm{H}_{2} \mathrm{O}\end{array}$ & $\begin{array}{l}\text { 2-Ketobutyric Acid } \\
\text { Ammonium } \\
\text { Cysteine } \\
\mathrm{P}_{\mathrm{i}} \\
\mathrm{PP}_{\mathrm{i}} \\
\mathrm{S} \text {-Adenosyl-L-Methionıne }\end{array}$ \\
\hline
\end{tabular}

Reaction: MET_CYS_1

$$
\begin{array}{rll}
\text { ATP } & \text { 2.5.1.6 } & \begin{array}{l}
\mathrm{Pi}_{\mathrm{i}} \\
\text { 4-Methionine }
\end{array} \\
\mathrm{H}_{2} \mathrm{O} & & \mathrm{PP}_{\mathrm{i}} \\
\mathrm{S}-\text { Adenosyl-L-Methionine }
\end{array}
$$

2.5.1.6: Methionine Adenosyltransferase

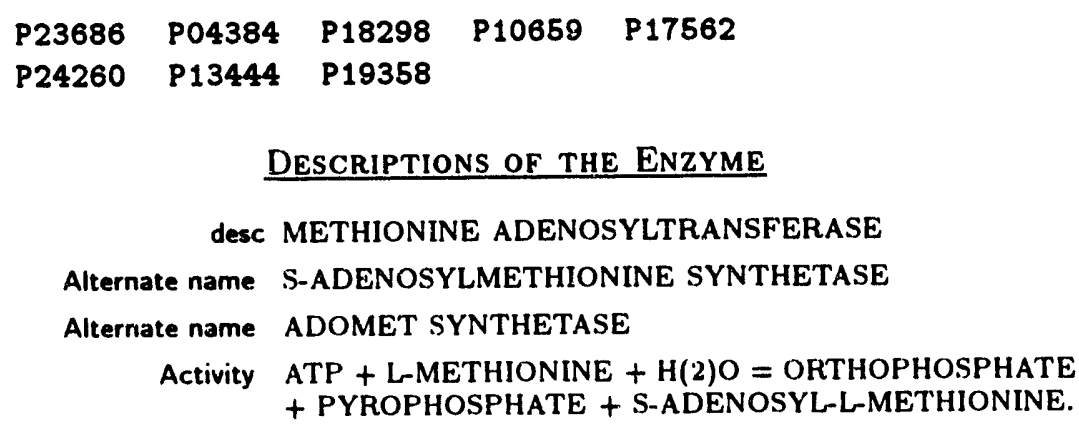

DESCRIPTIONS OF THE ENZYME

desc METHIONINE ADENOSYLTRANSFERASE

Alternate name S-ADENOSYLMETHIONINE SYNTHETASE

Alternate name ADOMET SYNTHETASE

Activity ATP + L-METHIONINE + H(2)O = ORTHOPHOSPHATE + PYROPHOSPHATE + S-ADENOSYL-L-METHIONINE.

P04384 metK 63.603105196 to 3106350

\section{DESCRIPTIONS OF THE PEPTIDE}

dese S-ADENOSYLMETHIONINE SYNTHETASE (EC 2.5.1.6) (METHIONINE ADENOSYLTRANSFERASE).

E.coli gene METK

\section{DESCRIPTIONS OF THE CDS}

desc Methionine adenosyltransferase 
Reaction: Homo-CYS_CYS_1

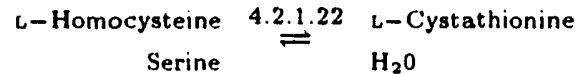

4.2.1.22: Cystathionine $\beta$-Synthase.

DESCRIPTIONS OF THE ENZYME

desc CYSTATHIONINE BETA-SYNTHASE

Alternate name SERINE SULFHYDRASE

Alternate name BETA-THIONASE

Alternate name METHYLCYSTEINE SYNTHASE

Activity L-SERINE + L-HOMOCYSTEINE = CYSTATHIONINE + $\mathrm{H}(2) \mathrm{O}$.

Pep??? cds??? Pos??? Loc???

Reaction: L-Cystathionine_CYS_1

\begin{tabular}{rll|}
\hline L-Cystationine Pyridoxal Phosphate & $\begin{array}{l}\text { 2-1.1.1 Ketobutyric Acid } \\
\mathrm{H}_{2} \mathrm{O}\end{array}$ & $\begin{array}{l}\text { Ammonium } \\
\text { Cysteine }\end{array}$ \\
\hline
\end{tabular}

4.4.1.1: Cystathionine $\gamma$-Lyase

DESCRIPTIONS OF THE ENZYME

desc CYSTATHIONINE GAMMA-LYASE

Alternate name HOMOSERINE DEAMINASE

Alternate name HOMOSERINE DEHYDRATASE

Alternate name GAMMA-CYSTATHIONASE

Alternate name CYSTINE DESULFHYDRASE

Alternate name CYSTEINE DESULFHYDRASE

Alternate name CYSTATHIONASE

Activity L-CYSTATHIONINE + H(2)O = L-CYSTEINE + $\mathrm{NH}(3)+$ 2-OXOBUTANOATE.

Pep??? metC 64.993171650 to 3172837

\section{DESCRIPTIONS OF THE CDS}

desc Cystathionine gamma-lyase 


\section{Summary of the Pathway: ETC_1}

\begin{tabular}{|c|c|c|}
\hline & $\begin{array}{l}1.6 .99 .3 \\
3.6 .1 .34\end{array}$ & \\
\hline $3.00 *$ ADP & $\begin{array}{c}\text { Cytochrome } B_{555} \\
\text { Cytochrome } B_{558} \\
\text { Cytochrome D } \\
\text { CytochromeO }\end{array}$ & \\
\hline $\mathrm{NADH}_{2}$ & $\begin{array}{c}6.00=H^{+} \\
3.00 * \text { Coenzyme } Q\end{array}$ & $3.00 *$ ATP \\
\hline $0.50 * \mathrm{O}_{2}$ & & NAD \\
\hline $3.00=P_{i}$ & & $\mathrm{H}_{2} \mathrm{O}$ \\
\hline $2.00 * \mathrm{H}^{+}$ & & \\
\hline
\end{tabular}

Reaction: ComplexI_1

$$
\begin{array}{rll}
\mathrm{NADH}_{2} & 1.6 .99 .3 & \mathrm{NAD} \\
2.00 * \mathrm{H}^{+} & = & 2.00 * \mathrm{H}^{+}(\text {external) } \\
\text { Coenzyme Q } & \mathrm{CoQ}-2 \mathrm{H}
\end{array}
$$

$\begin{array}{lllll}\text { 1.6.99.3: NADH Dehydrogenase } & & \\ \text { P11943 } & \text { P } 00393 & \text { P15690 } & \text { P24918 } & \text { P25708 } \\ \text { P24917 } & \text { P17694 } & \text { P22142 } & \text { P25284 } & \text { P24919 } \\ \text { P23709 } & \text { P23710 } & \text { P04394 } & \text { P19404 } & \text { P19234 } \\ \text { P19968 } & \text { P25710 } & \text { P21976 } & \text { P25711 } & ? 23934 \\ \text { P23935 } & \text { P25712 } & & & \end{array}$

DESCRIPTIONS OF THE ENZYME

desc NADH DEHYDROGENASE

Alternate name CYTOCHROME C REDUCTASE

Alternate name TYPE I DEHYDROGENASE

Activity $\mathrm{NADH}+$ ACCEPTOR $=\mathrm{NAD}(+)+$ REDUCED ACCEPTOR.

P00393 ndh 22.491176653 to 1177957

\section{DESCRIPTIONS OF THE PEPTIDE}

desc NADH DEHYDROGENASE (EC 1.6.99.3)

E.coli gene NDH

\section{DESCRIPTIONS OF THE CDS}


desc Respiratory NADH dehydrogenase

\section{Reaction: Cyt-b556_1}

$$
\begin{aligned}
\text { CytochromeB } 556 \\
\text { CoQ-2H }
\end{aligned} \Longrightarrow \begin{aligned}
& \text { CytochromeB } 556-2 \mathrm{e}^{-} \\
& 2.00 \mathrm{H}^{+}(\text {external) } \\
& \text { Coenzyme Q }
\end{aligned}
$$

No enzymes have been found to catalyze this reaction.

\section{Reaction: Cyt-b558_1}

$$
\begin{aligned}
& \text { CytochromeB } \begin{array}{l}
\text { CytochromeB } \\
\text { CoQs }-2 \mathrm{e}^{-}
\end{array} \\
& 2.00 \mathrm{H}^{+}(\text {external }) \\
& \text { Coenzyme } \mathrm{Q}
\end{aligned}
$$

No enzymes have been found to catalyze this reaction.

\section{Reaction: Cyt-b556-2e_1}

$$
\begin{array}{r}
\text { CytochromeB } 556-2 \mathrm{e}^{-} \\
\text {CytochromeO }
\end{array} \text { CytochromeB } 556_{\text {CytochromeO-2e- }}
$$

No enzymes have been found to catalyze this reaction.

\section{Reaction: Cyt-b558-2e_1}

$$
\begin{array}{r}
\text { CytochromeB } \\
\text { Cysto-2 } \mathrm{e}^{-}
\end{array} \rightleftharpoons \begin{aligned}
& \text { CytochromeB } \\
& \text { Cys } \\
& \text { Cytochromed }
\end{aligned}
$$

No enzymes have been found to catalyze this reaction.

\section{Reaction: Cyt-D-2e_1}

$$
\begin{aligned}
\text { Cytochromed }-2 \mathrm{e}^{-} \\
0.50 * \mathrm{O}_{2} \\
2.00 * \mathrm{H}^{+}
\end{aligned} \Longrightarrow \begin{aligned}
& \text { CytochromeD } \\
& \mathrm{H}_{2} \mathrm{O}
\end{aligned}
$$

No enzymes have been found to catalyze this reaction. 
Reaction: Cyt-0-2e_1

$$
\begin{aligned}
\text { CytochromeO-2e- } \\
0.50 * \mathrm{O}_{2} \\
2.00 * \mathrm{H}^{+}
\end{aligned}=\begin{aligned}
& \text { CytochromeO } \\
& \mathrm{H}_{2} \mathrm{O}
\end{aligned}
$$

No enzymes have been found to catalyze this reaction.

\begin{tabular}{|c|c|c|}
\hline $\begin{array}{r}\text { ADP } \\
P_{i} \\
2.00 * \mathrm{H}^{+}(\text {external })\end{array}$ & $\stackrel{3.6 .1 .34}{=}$ & $\begin{array}{l}\text { ATP } \\
2.00 * \mathrm{H}^{+}\end{array}$ \\
\hline
\end{tabular}

\section{Reaction: ADP_1}

3.6.1.34: $\mathrm{H}^{+}$-Transporting ATP Synthase 


\begin{tabular}{|c|c|c|c|c|}
\hline P19483 & P22201 & P18260 & P05494 & P05495 \\
\hline P05492 & P 15998 & P05493 & P24459 & P80021 \\
\hline P23413 & P80082 & P12862 & P19023 & P17614 \\
\hline P80083 & P26360 & P80084 & P80085 & P12404 \\
\hline P24876 & $\mathrm{P} 00853$ & P00852 & P25965 & P22476 \\
\hline P20600 & P24945 & P00847 & P24888 & $\mathrm{P} 14092$ \\
\hline P14862 & $\mathrm{P}_{14413}$ & P34946 & P00850 & P00851 \\
\hline P00855 & $\mathrm{P} 00846$ & $\mathrm{P} 14569$ & P07925 & P00848 \\
\hline P22067 & P05500 & P1 2696 & P25005 & P15994 \\
\hline P25761 & $\mathrm{P} 05504$ & P15012 & P21535 & $\mathrm{P} 80086$ \\
\hline P15995 & P08444 & P09218 & P05499 & P24499 \\
\hline P12984 & P20599 & P00849 & P00854 & P80088 \\
\hline P00858 & $\mathrm{P} 00857$ & P24947 & P03929 & P05040 \\
\hline P17345 & P14093 & P14414 & P 24948 & P03932 \\
\hline P03933 & P15996 & P03928 & P14570 & 949 \\
\hline P03930 & P07513 & P12697 & 004 & P11608 \\
\hline P21536 & P15997 & P03931 & 356 & 571 \\
\hline P17254 & $\mathrm{P} 00840$ & P14572 & P14863 & 001 \\
\hline P05717 & P21537 & P05498 & P13547 & 841 \\
\hline P12405 & P22477 & P17674 & P19482 & 822 \\
\hline P25163 & P25705 & P05022 & 283 & 662 \\
\hline P12084 & P08215 & P15999 & P05439 & 036 \\
\hline P24487 & P06450 & P09639 & P08.149 & 219 \\
\hline P00823 & P12985 & $P_{12112}$ & P08428 & 251 \\
\hline P06540 & P19366 & P22478 & 356 & 698 \\
\hline P00829 & P06541 & P13357 & P00824 & 164 \\
\hline P00828 & P06576 & P07137 & P00827 & 284 \\
\hline P22663 & P20022 & P23704 & P12085 & 037 \\
\hline P10719 & P05440 & P05038 & 068 & 825 \\
\hline P21933 & P13052 & РОт890 & 677 & 826 \\
\hline P12986 & P20858 & P00830 & P12406 & 479 \\
\hline P17675 & P05630 & POC & 437 & 38 \\
\hline P11402 & P08448 & PO & P12987 & P05626 \\
\hline P06542 & P09468 & P22 & P12699 & 332 \\
\hline P07891 & P00832 & P00836 & P07138 & 335 \\
\hline P06285 & P12086 & P05039 & P05441 & 442 \\
\hline P00833 & P07892 & P07678 & P00834 & 988 \\
\hline P20859 & P21306 & P12407 & P22481 & P20601 \\
\hline$P_{13619}$ & P00859 & P24539 & P06291 & 2087 \\
\hline P08214 & P19511 & $P_{15013}$ & P06453 & 3447 \\
\hline P09221 & P06290 & P12989 & P06528 & .108 \\
\hline P22 & P20602 & P05631 & P12113 & P00837 \\
\hline P05 & POT227 & P05435 & $\mathbf{P O}$ & 253 \\
\hline POQ & P12 & $\mathrm{P} 10$ & & \\
\hline$P_{12}$ & $\mathrm{POC}$ & $\mathrm{PO}$ & 44 & \\
\hline P 2083 & P06452 & POG & 288 & 109 \\
\hline P16000 & P25966 & P22483 & P20603 & 839 \\
\hline P00844 & P05496 & P00842 & P15014 & P17605 \\
\hline P23040 & P08445 & P00845 & P12991 & P07926 \\
\hline P13621 & P22778 & $P_{17604}$ & P80087 & P09.157 \\
\hline$P 13620$ & P02721 & P18859 & P13618 & P21571 \\
\hline P12410 & P25075 & $P_{15015}$ & P08446 & P23445 \\
\hline $\mathrm{P} 22$ & P23039 & P22721 & P09469 & P11592 \\
\hline$P_{13548}$ & P17255 & P11574 & P22550 & P15313 \\
\hline P11593 & P16140 & P21281 & P21 282 & P21283 \\
\hline P11019 & P22203 & P12953 & P23957 & P2395 \\
\hline P23380 & P23967 & P25515 & & \\
\hline
\end{tabular}




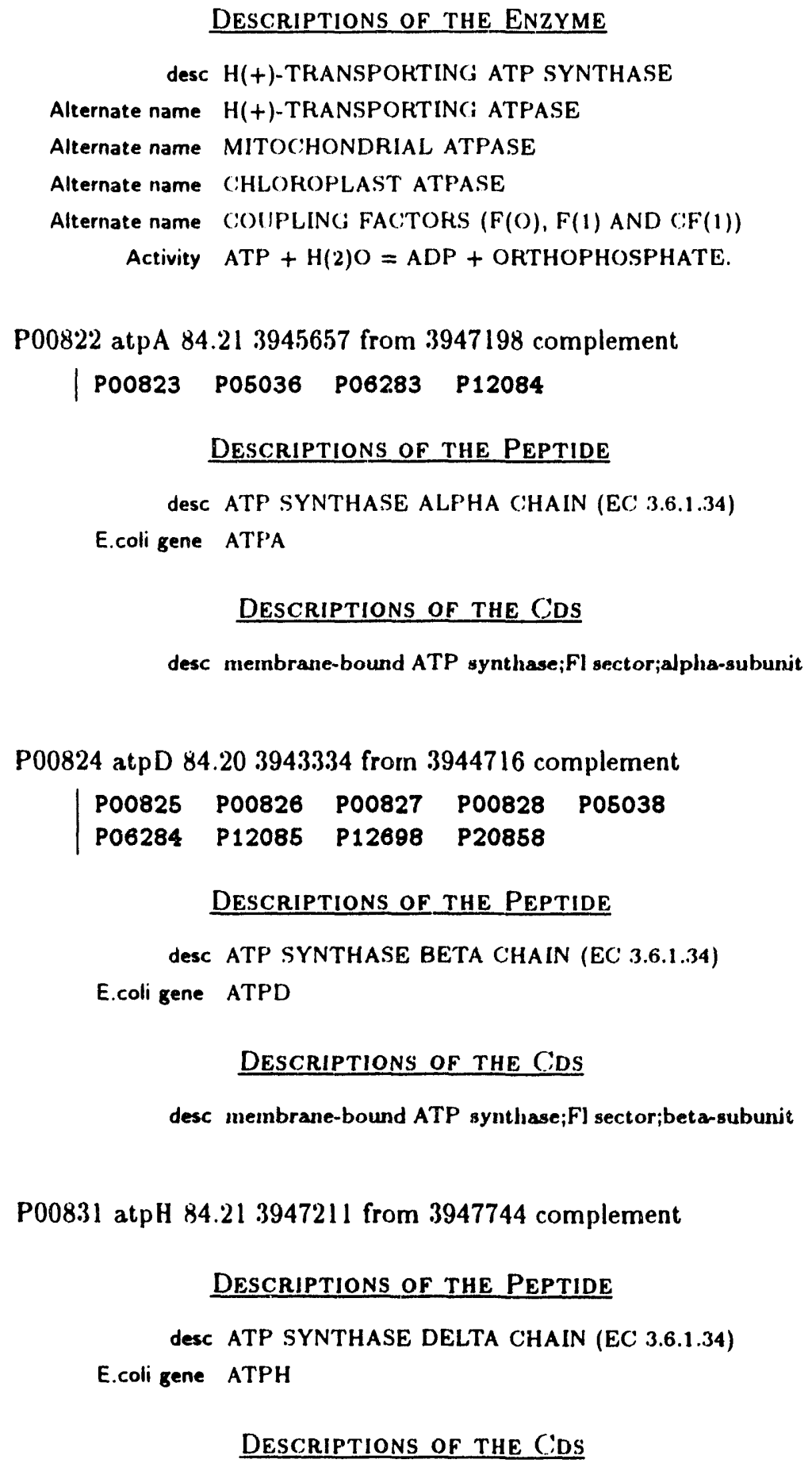


desc membrane-bound ATP synthase; Fl sector;delta-subunit

P00832 atp(: 84.203942894 from 3943313 complement

\section{DESCRIPTIONS OF THE PEPTIDE}

desc ATP SYNTHASE EPSILON CHAIN (EC 3.6.1.34)

E.coli gene ATPC:

DESCRIPTIONS OF THE CDS

desc membrane-bound ATP synthase; F1 sector;epsilon subunit

P008:37 atp(i 84.203944743 from 3945606 complement

Descriptions of the Peptide

desc ATP SYNTHASE GAMMA CHAIN (EC 3.6.1.34)

E.coli gene ATPGi

DESCRIPTIONS OF THE CDS

desc membrane-bound ATP synthase;F1 sector;gamma-subunit

P00844 atpE 84.213948291 from 3948530 complement

Descriptions of The Peptide

desc ATP SYNTHASE C CHAIN (EC 3.6.1.34) (LIPID-BINDING: PROTFIN) (DICYCLOHEXYLCARBODIIMIDE-BINDING PROTEIN).

E.coli gene ATPE

DESCRIPTIONS OF THE CDS

desc inembrane-bound ATP synthase;Fo sector;subunit c;DCCDbinding protein

P00855 atpB 84.213948577 from 3949392 complement

DESCRIPTIONS OF THE PEPTIDE 
desc ATP SYNTHASE A CHAIN (EC 3.6.1.34) (PROTEIN 6)

E.coli gene ATPB

DESCRIPTIONS OF THE CDS

desc membrane-bound ATP synthase;Fo sector;subunit a

P00859 atpF 84.213947759 from 3948229 complement

DESCRIPTIONS OF THE PEPTIDE

desc ATP SYNTHASE B CHAIN (EC 3.6.1.34)

E.coli gene ATPF

DESCRIPTIONS OF THE CDS

desc membrane-bound ATP synthase;Fo sector;subunit b 


\section{Summary of the Pathway: ETC_2}

\begin{tabular}{|c|c|c|}
\hline & $\begin{array}{r}1.3 .99 .1 \\
3.6 .1 .34\end{array}$ & \\
\hline $2.00 * A D P$ & $\begin{array}{c}\text { Cytochrome } B_{536} \\
\text { Cytochrome } B_{538} \\
\text { CytochromeD } \\
\text { CytochromeO }\end{array}$ & \\
\hline $\mathrm{FADH}_{2}$ & $4.00 * \mathrm{H}^{+}$ & $2.00 * \mathrm{ATP}$ \\
\hline $0.50 * \mathrm{O}_{2}$ & $2.00 *$ Coenzyme $Q$ & FAD \\
\hline $2.00 * \mathrm{P}_{\mathrm{i}}$ & & Fumarate \\
\hline $2.00 * \mathrm{H}^{+}$ & & $\mathrm{H}_{2} \mathrm{O}$ \\
\hline Succinate & & \\
\hline
\end{tabular}

Reaction: ComplexII_1

$\begin{array}{rll}\mathrm{FADH}_{2} & \text { 1.3.99.1 } & \text { FAD } \\ \text { Succinate } & \rightleftharpoons & \text { Fumarate } \\ \text { Coenzyme Q } & & \text { CoQ-2H }\end{array}$

1.3.99.1: Succinate Dehydrogenase

$\begin{array}{lllll}\text { P08065 } & \text { P10444 } & \text { P08066 } & \text { P07014 } & \text { P08064 } \\ \text { P10446 } & \text { P00363 } & \text { P20922 } & \text { P17412 } & \text { P00364 } \\ \text { P20921 } & \text { P17596 } & \text { P17413 } & & \end{array}$

DESCRIPTIONS OF THE ENZYME

desC SUCCINATE DEHYDROGENASE

Alternate name SUCCINIC DEHYDROGENASE

Alternate name FUMARATE REDUCTASE

Alternate name FUMARATE DEHYDROGENASE

Alternate name FUMARIC HYDROGENASE

Activity SUCCINATE + ACCEPTOR = FUMARATE + REDUCED ACCEPTOR.

P00363 frdA 94.464412246 from 4414054 complement

\section{Descriptions OF THE PEPTIDE}

desC FUMARATE REDUCTASE FLAVOPROTEIN SUBUNIT (EC 1.3.99.1)

E.criigene FRDA 


\section{DESCRIPTIONS OF THE CDS}

desc Funarate reductase flavoprotein subunit

P00364 frdB 94.454411519 from 4412253 complement

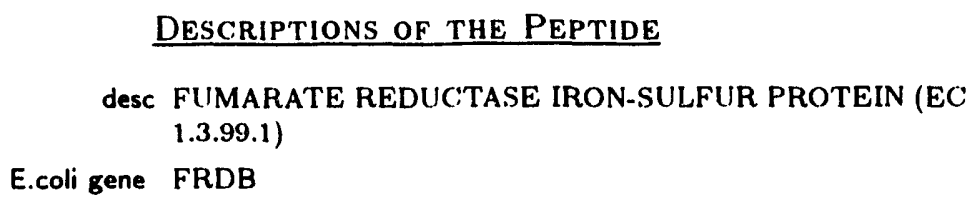

\section{DESCRIPTIONS OF THE CDS}

desc Fumarate reduct ase iron-sulfur protein subunit

P07014 sdhB 16.50767892 to 768608

\section{DESCRIPTIONS OF THE PEPTIDE}

desc SUCCINATE DEHYDROGENASE IRON-SULFUR PROTEIN (EC 1.3.99.1)

E.coli gene SDHB

\section{DESCRIPTIONS OF THE CDS}

desc Succinate dehydrogenase; iron sulfur protein

P10444 sdhA 16.50766110 to 767876

\section{Descriptions of the Peptide}

desc SUCCINATE DEHYDROGENASE FLAVOPROTEIN SUBUNIT (EC 1.3.99.1)

E.coli gene SDHA

DESCRIPTIONS OF THE CDS

desc Succinate dehydrogenase; flavoprotein subunit

P10446 sdhC: 16.50765380 to 765769 


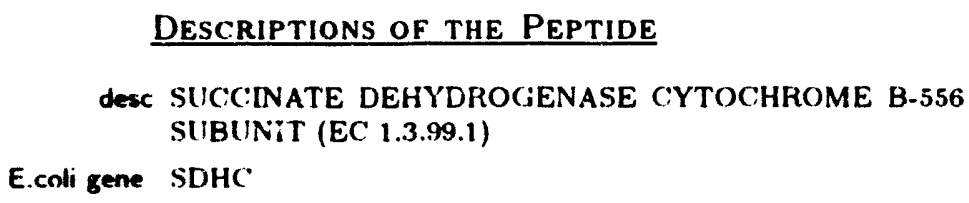

DEsCRIPTIONS OF THE PEPTIDE

desc SUCC:INATE DEHYDROGENASE CYTOCHROME B-556 SUBUNIT (EC 1.3.99.1)

E.coli gene SDHC

\section{DESCRIPTIONS OF THE C'DS}

desc succinate dehydrogenase; cytochrome b556

Reaction: "yt-b556_1

$$
\begin{aligned}
& \text { CytochromeBsse CytochromeBsse-2e } \\
& \left(\circ \mathrm{QQ}-2 \mathrm{H}=2.00 * \mathrm{H}^{+}\right. \text {(external) } \\
& \text { Coenzyme } Q
\end{aligned}
$$

No enzymes have been found to catalyze this reaction.

Reaction: Cyt-b558_1

$$
\begin{aligned}
\text { CytochromeBss }_{s s} \\
\text { CoQ-2H }
\end{aligned}=\begin{aligned}
& \text { CytochromeB } \\
& 2.00 \cdot \mathrm{H}^{+}(\text {external }) \\
& \text { Coenzyme } \mathrm{Q}
\end{aligned}
$$

No enzymes have heen found to catalyze this reaction.

Reaction: Cyt-b556-2e__1

$$
\underset{\text { CytochromeO }}{{\text { CytochromeBss }-2 e^{-}}_{\text {CytochromeO-2e- }}^{-}}
$$

No enzymes have been found to catalyze this reaction.

Reaction: Cyt-b558-2e_1

$$
\begin{aligned}
& \text { CytochromeBsse-2e- }^{-}=\text {CytochromeBsse }_{\text {sse }} \\
& \text { Cytochromed }=\text { Cytochromed-2e- }
\end{aligned}
$$

No enzymes have been found to catalyze this reaction. 
Reaction: Cyt-D-2e_1

$$
\begin{aligned}
\text { Cyeochrome } \mathrm{O}-2 \mathrm{e}^{-} \\
050 . \mathrm{O}_{2} \\
2000 \mathrm{H}^{+}
\end{aligned}=\begin{aligned}
& \text { CyeochromeD } \\
& \mathrm{H}_{2} \mathrm{O}
\end{aligned}
$$

No enzymes have been found to catalyze this reaction.

Reaction: Cyt-0-2e_1

$$
\begin{aligned}
\text { (ytochrome }-2 e^{-} \\
050 . \mathrm{O}_{2} \\
200 \cdot \mathrm{H}^{+}
\end{aligned}=\begin{aligned}
& \text { GechromeO } \\
& \mathrm{H}_{2} \mathrm{O}
\end{aligned}
$$

No enzymes have been found to catalyze this reaction.

\section{Reaction: ADP_1}

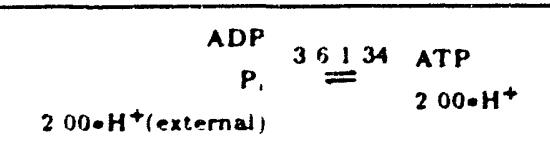

3.6.1.34: $\mathrm{H}^{+}$-Transporting ATP Synthase 


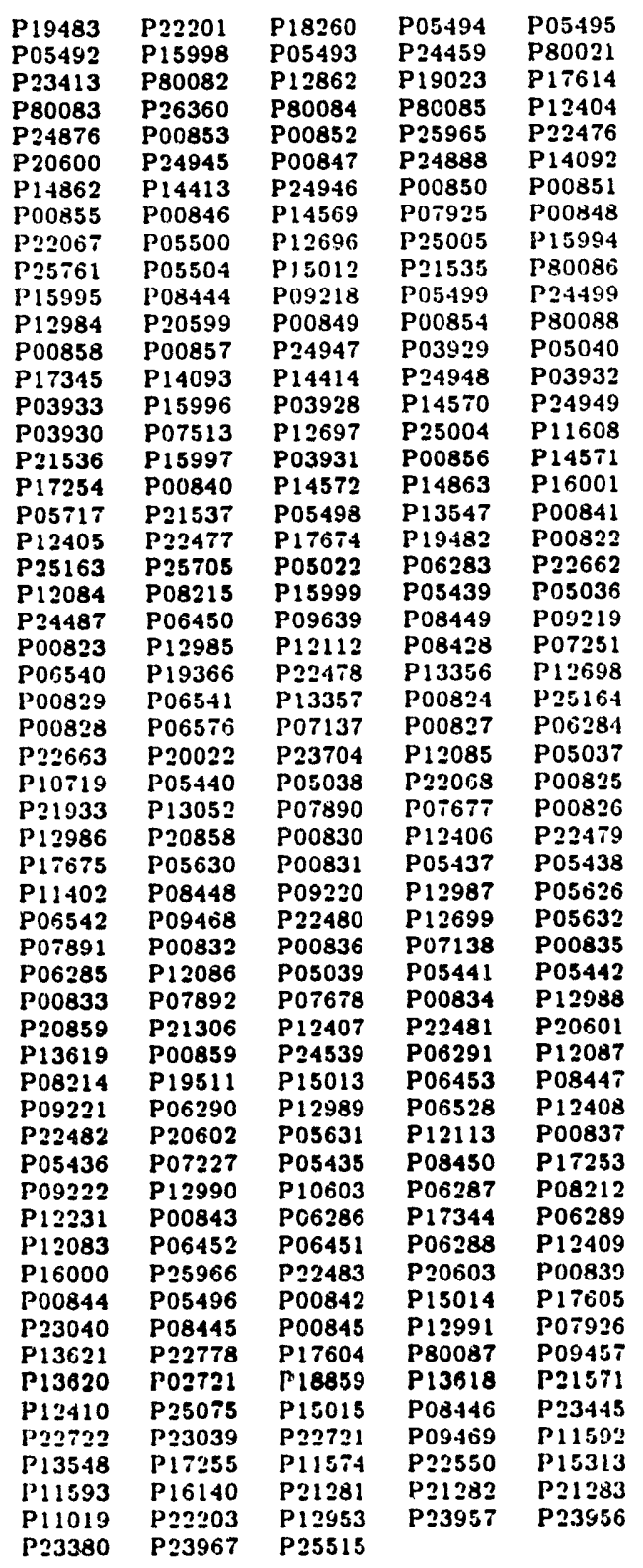




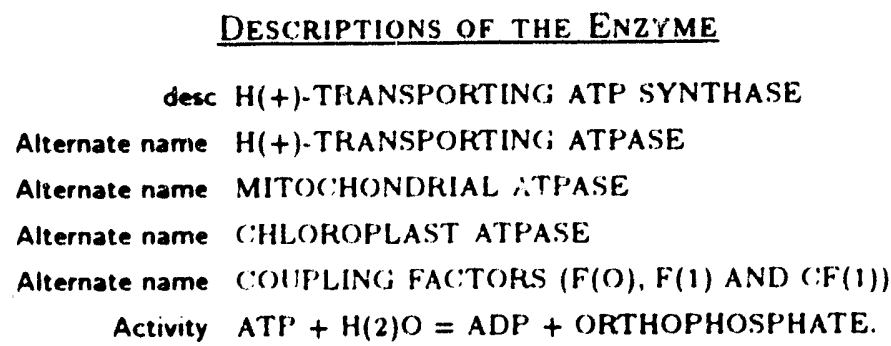

P008.31 atpH 84.213947211 from 3947744 complement

DESCRIPTIONS OF THE PEPTIDE

desc ATP SYNTHASE DELTA CHAIN (EC 3.6.1.34)

E.coli gene ATPH

DESCRIPTIONS OF THE CIOS 
desc membrane-bound ATP synthase; Fl sector;delta-subunit

P00832 atp(: 84.203942894 from 3943313 complement

DESCRIPTIONS OF THE PEPTIDE

desc ATP SYNTHASE EPSLLON CHAIN (EC 3.6.1.34)

E.coli gene ATPC

DESCRIPTIONS OF THE CDS

desc membrane-bound ATP synthase;F1 sector;epsilon subunit

P00837 atp(i 84.203944743 from 3945606 complement

\section{DESCRIPTIONS OF THE PEPTIDE}

desc ATP SYNTHASE GAMMA CHAIN (EC 3.6.1.34)

E.coli gene ATPG

\section{DESCRIPTIONS OF THE C.DS}

desc membrane-bound ATP synthase;F1 sector;ganuna-subunit

P00844 atpE 84.213948291 from 3948530 complement

DESCRIPTIONS OF THE PEPTIDE

desc ATP SYNTHASE C CHAIN (EC 3.6.1.34) (LIPID-BINDINC PROTEIN) (DICYCLOHEXYLCARBODIIMIDE-BINDING: PROTEIN).

E.coli gene ATPE

DESCRIPTIONS OF THE C.DS

desc membrane-bound ATP synthase;Fo sector;subunit c;DC:CDbinding protein

P00855 atpB 84.213948577 from 3949392 complement

Descriptions of the Peptide 


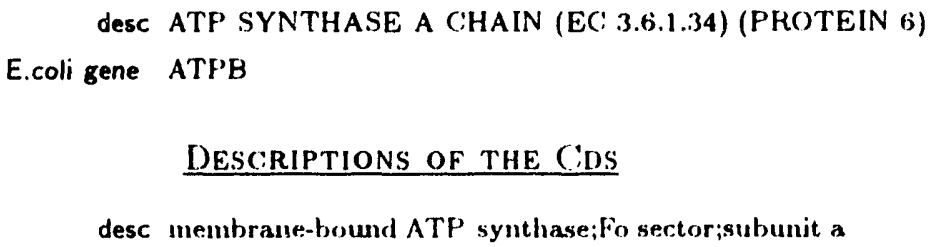




\section{Summary of the Pathway: FAD-Synthesis_1}

$\begin{array}{lcl}2.00 * \text { ATP } & 2.7 .1 .26 & \text { ADP } \\ \text { Riboflavin } & & \text { FAD } \\ & & \text { PPi }_{i}\end{array}$

Reaction: Riboflavin_FAD_1

\begin{tabular}{|c|c|c|}
\hline ATP & $\stackrel{2.7 .1 .26}{\rightleftharpoons}$ & ADP \\
\hline Riboflavin & & FMN \\
\hline
\end{tabular}

2.7.1.26: Riboflavin Kinase

\section{DESCRIPTIONS OF THE ENZYME}

desc RIBOFLAVIN KINASE

Alternate name FLAVOKINASE

Activity ATP + RIBOFLAVIN = ADP + FMN.

Pep??? cds??? Pos??? Loc???

Reaction: FMN_FAD_1

$\begin{aligned} \mathrm{ATP} & \stackrel{2.7 .7 .2}{=} \mathrm{FAD} \\ \mathrm{FMN} & \stackrel{\mathrm{PP}_{\mathrm{i}}}{=}\end{aligned}$

2.7.7.2: FMN Adenylyltransferase

DESCRIPTIONS OF THE ENZYME

desc FMN ADENYLYLTRANSFERASE

Alternate name FAD PYROPHOSPHORYLASE

Activity ATP + FMN = PYROPHOSPHATE + FAD.

Pep??? cds??? Pos??? Loc??? 


\section{Summary of the Pathway: Fat-Degradation_1}

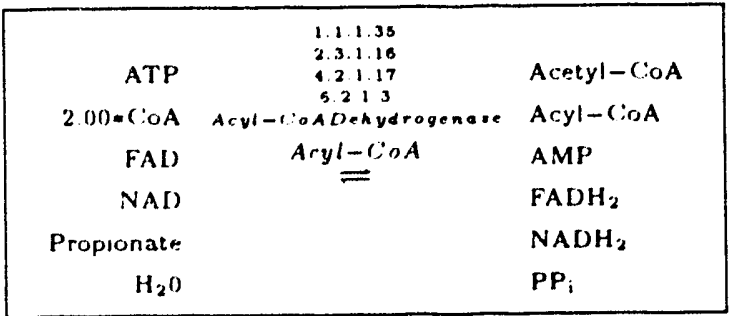

Reaction: fatty acid_1

$$
\begin{array}{rl}
\mathrm{ATP} & 6.213 \\
\operatorname{CoA} \stackrel{\mathrm{A} \subseteq \mathrm{yl}-\mathrm{CoA}}{\mathrm{AMP}} \\
\mathrm{PP}_{\mathrm{i}}
\end{array}
$$

6.2.1.3: Long-Chain-Fatty-Acıd-CoA Ligase

\section{DESCRIPTIONS OF THE ENZYME}

desc LONG-CHAIN-FATTY-ACID-COA LIGA.SE

Alternate name ACYL-ACTIVATING ENZYME

Alternate name ACYL-COA. SYNTHETASE

Alternate name FATTY ACID THIOKINASE (LONG-CHAIN)

Alternate name LIC;NOCEROYL-COA SYNTHASE

Activity ATP + A LONG-CHAIN CARBOXYLIC ACID + COA = AMP + PYROPHOSPHATE + AN ACYLCOA.

Pep??? fadD 40.00 Loc???

\section{DESCRIPTIONS OF THE CDS}

desc acyl-CoA synthetase

Reaction: Acyl-CoA_1

$$
\begin{aligned}
\text { Acyl-COA Acyl-CoADehydrogenase } & \text { Enoyl-CoA } \\
\text { FAD } & \mathrm{FADH}_{2}
\end{aligned}
$$

Acyl-CioA Dehydrogenase. 
Pep??? cds??? Pos??? Loc???

Reaction: Enoyl-CoA_1

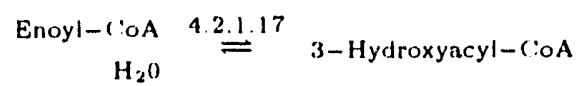

4.2.1.17: Enoyl-CoA Hydratase

P14604 P07896 P21177

\section{DESCRIPTIONS OF THE ENZYME}

desc ENOYL-COA HYDRATASE

Alternate name ENOYL HYDRASE

Alternate name IINSATURATED ACYL-COA HYDRATASE

Activity (3S)-3-HYDROXYACYL-COA = TRANS-2(OR 3)-ENOYLCOA $+\mathrm{H}(2) \mathrm{O}$.

P'1177 fadB 86.994058085 from 4060274 complement

\section{DESCRIPTIONS OF THE PEPTIDE}

desc FATTY OXIDATION COMPLEX ALPHA SUBUNIT (CONTAIN: ENOYL-COA HYDRATASE (EC 4.2.1.17), DELTA(3)CIS-DELTA(2)-TRANS-ENOYL-COA ISOMERASE (EC 5.3.3.8), 3-HYDROXYACYL-COA DEHYDROGENASE (EC 1.1.1.35), AND 3- HYDROXYBUTYRYL-COA EPIMERASE (EC; 5.1 .2 .3 ).

E.coli gene FADB

\section{DESCRIPTIONS OF THE CDS}

desc 3-hydroxyacyl-CoA dehydrogenase, 3-hydroxyacyl-CoA epimerase;delta3Cl5-delta2-trans-enoyl-CoA and enoyl-CoA and enoyl-CoAhydratase (crotonase)

Reaction: 3-Hydroxy Acyl-CoA_1

$$
\begin{aligned}
& 3-\text { Hydroxyacyl-CoA } \stackrel{1.1 .35}{=} 3-\text { Ketoacyl-CoA }_{\text {NAD }} \\
& \mathrm{NADH}_{2}
\end{aligned}
$$

1.1.1.35: 3-Hydroxyacyl-CoA Dehydrogenase

$\begin{array}{lll}\text { P07896 P21177 P00348 } & \text { P2 }\end{array}$ 


\section{DESCRIPTIONS OF THE ENZYME}

desc 3-HYDROXYACYLCOA DEHYDROCIENASE

Alternate name BETA-HYDROXYAC'YL DEHYDROCIENASE

Alternate name BETA-KETO-REDUCTASE

Activity L-3-HYDROXYAC:YL-C:OA + NAD(+)=3-OXOAC:YL-COA + NADH.

P'21177 fadB 86.994058085 from 4060274 complement

\section{DESCRIPTIONS OF THE PEPTIDE}

desc FATTY OXIDATION COMPLEX ALPHA SUBUNIT (CONTAIN: ENOYL-COA HYDRATASE (EC 4.2.1.17), DELTA(3)CIS-DELTA(2)-TRANS-ENOYL-COA ISOMERASE (EC 5.3.3.8), 3-HYDROXYACYL-COA DEHYDROCENASE (EC; 1.1.1.35), AND 3- HYDKOXYBUTYRYL-COA EPIMERASE (EC: 5.1.2.3).

E.coli gene FADB

\section{DESCRIPTIONS OF THE CDS}

desc 3-hydroxyacyl-CoA dehydrogenase, 3-hydroxyacyl-CoA epimerase;delta3Cl15-delta2-trans-enoyl-CoA and enoyl-CoA and enoyl-CoAhydratase (crotonase)

Reaction: 3-KetoAcyl-CoA_1

$$
\begin{aligned}
& \text { 3-Ketoacyl-CoA 2.3.1.16 Acetyl-CoA } \\
& \mathrm{CoA} \text { Acyl-CoA }
\end{aligned}
$$

2.3.1.16: Acetyl-Coa Acyltransferase

$\begin{array}{lllll}\text { P07871 } & \text { P21775 } & \text { P21151 } & \text { P09110 } & \text { P13437 }\end{array}$

DESCRIPTIONS OF THE ENZYME

desc ACETYLCOA ACYLTRANSFERASE

Alternate name BETA-KETOTHIOLASE

Alternate name 3-KETOACYLCOA THIOLASE

Activity AC:YL-COA + ACETYL-COA = COA + 3-OXOACYL-COA.

P21151 fadA 86.994056912 from 4058075 complement 


\section{Descriptions of the PEPTIDE}

desc 3-KETOACYL-COA THIOLASE (EC 2.3.1.16) (FATTY OXIDATION COMPLEX BETA SUBUNIT) (BETA-KETOTHIOLASE) (AC:ETYL-C:OA AC:YLTRANSFERASE).

E.coli gene FADA

\section{DESCRIPTIONS OF THE CDS}

desc thiolase I 


\section{Summary of the Pathway: GTP-Synthesis_1}

\begin{tabular}{|c|c|c|}
\hline $\begin{array}{r}3.00 * \text { ATP } \\
\text { (ilutamine } \\
\text { IMP } \\
\mathrm{NAD} \\
2.00 * \mathrm{H}_{2} \mathrm{O}\end{array}$ & $\begin{array}{c}1.1 .1 .205 \\
2.7 .4 .6 \\
2.7 .4 .8 \\
6.3 .5 .2 \\
\rightleftharpoons\end{array}$ & $\begin{array}{l}2.00 * \text { ADP } \\
\text { AMP } \\
\text { Cilutamate } \\
\text { GTP } \\
\mathrm{NADH}_{2} \\
\text { PPi }\end{array}$ \\
\hline
\end{tabular}

Reaction: IMP_Guanosine_1

\begin{tabular}{rll|}
$\begin{array}{r}\text { IMP } \\
\text { NAD }\end{array}$ & 1.1 .1 .205 & $\mathrm{NADH}_{2}$ \\
$\mathrm{H}_{2} \mathrm{O}$ & & Xanthosine-5-P \\
\hline
\end{tabular}

1.1.1.205: IMP Dehydrogenase

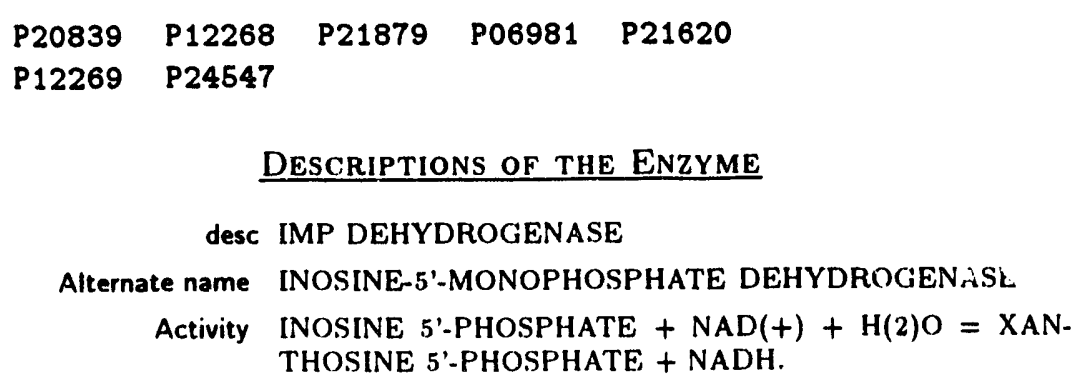

DESCRIPTIONS OF THE ENZYME

desc IMP DEHYDROGENASE

Alternate name INOSINE-5'-MONOPHOSPHATE DEHYDROGENASL

Activity INOSINE 5'-PHOSPHATE $+\mathrm{NAD}(+)+\mathrm{H}(2) \mathrm{O}=\mathrm{XAN}$ THOSINE 5'-PHOSPHATE + NADH.

P06981 guaB 53.90 2640554 from 2642020 complemenı

\section{Descriptions of THE PEPTIDE}

desC INOSINE-5'-MONOPHOSPHATE DEHYDROGENASE (EC 1.1.1.205) (IMP DEHYDROGENASE) (IMPDH).

E.coli gene GUAB

\section{DESCRIPTIONS OF THE CDS}

desc IMP dehydrogenase 
Reactin: Xanthosine-5-P_Guanosine_1

\begin{tabular}{|c|c|c|}
\hline ATP & & AMP \\
\hline $\begin{array}{r}\text { Glutamine } \\
\mathrm{H}_{2} \mathrm{O}\end{array}$ & $\stackrel{6.3 .5 .2}{\rightleftharpoons}$ & $\begin{array}{l}\text { Gilutamate } \\
\text { GMP }\end{array}$ \\
\hline Xanthosine $-5-P$ & & $\mathbf{P P} \mathbf{P}_{\mathbf{i}}$ \\
\hline
\end{tabular}

6.3.5.2: GMP Synthase (Glutamine-Hydrolysing) P04079

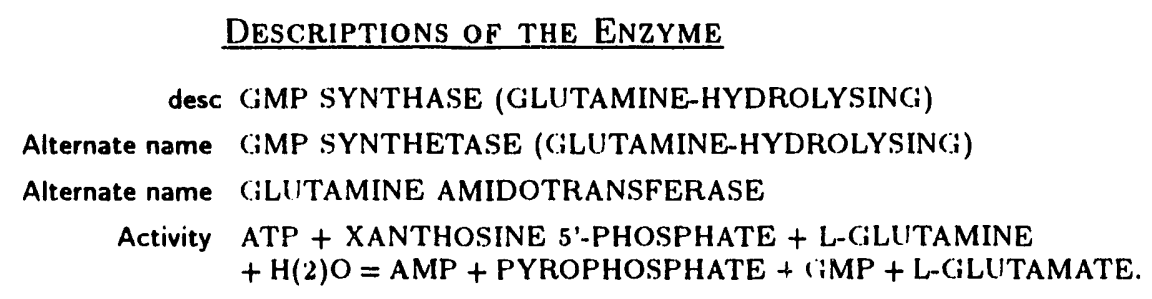

DESCRIPTIONS OF THE ENZYME

desc GMP SYNTHASE (GLUTAMINE-HYDROLYSING)

Alternate name GMP SYNTHETASE (GLUTAMINE-HYDROLYSING)

Alternate name GLUTAMINE AMIDOTRANSFERASE

Activity ATP + XANTHOSINE 5'-PHOSPHATE + L-GLUTAMINE $+\mathrm{H}(2) \mathrm{O}=$ AMP + PYROPHOSPHATE + (IMP + L-GiLUTAMATE.

P04079 guaA 53.902638908 from 2640485 complement

\section{DESCRIPTIONS OF THE PEPTIDE}

desc GMP SYNTHASE (GLUTAMINE-HYDROLYSING) (EC 6.3.5.2) (GLUTAMINE AMIDOTRANSFERASE).

E.coli gene GUAA

DESCRIPTIONS OF THE CDS

desc (iMP synthetase

Reaction: GMP_Guanosine_1

$$
\begin{array}{rrr}
\text { ATP } & 2.7 .4 .8 & \text { ADP } \\
\text { GMP } & & \text { GDP }
\end{array}
$$

2.7.4.8: Guanylale Kinase

\section{DESCRIPTIONS OF THE ENZYME}

desc GUANYLATE KINASE

Alternate name DEOXYGUANYLATE KINASE

Activity $\mathrm{ATP}+\mathrm{GMP}=\mathrm{ADP}+\mathrm{GDP}$. 
Pep??? cds??? Pos??? Loc???

\section{Reaction: GDP_Guanosine.1}

$$
\begin{aligned}
& \text { ATP 2.7.4.6 ADP } \\
& \text { GDP } \rightleftharpoons \text { (ITP }
\end{aligned}
$$

\subsubsection{6: Nucleoside-Diphosphate Kinase}

\section{DESCRIPTIONS OF THE ENZYME}

desc NUCLEOSIDE-DIPHOSPHATE KINASE

Alternate name NUCLEOSIDE 5 '-DIPHOSPHATE PHOSPHOTRANSFERASE

Alternate name NDK

Activity ATP + NUCLEOSIDE DIPHOSPHATE $=$ ADP + NUCLEOSIDE TRIPHOSPHATE.

Pep??? cds??? Pos??? Loc??? 


\section{Summary of the Pathway: Gluconeogenesis_1}

\begin{tabular}{|c|c|c|}
\hline $\begin{array}{r}(1,4-\alpha-D-(\text { ilucosyl). } \\
700 \times \text { ATP } \\
2.00 \text {. Pyruvate }\end{array}$ & 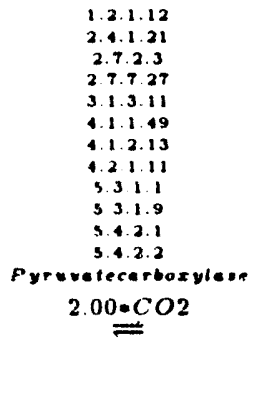 & $\begin{array}{l}(1,4-\alpha-D-(\text { ilucosyl) }=+1 \\
7,00 * A D P \\
300 * P_{i} \\
\because\end{array}$ \\
\hline
\end{tabular}

Reaction: pyruvate_3

$\begin{array}{rll}\text { ATP } & \text { Pyruvatecarbosylase } & \text { ADP } \\ \mathrm{CO}_{2} & \stackrel{\text { Oxalacetic Acid }}{=} & \mathrm{Pi}_{\mathrm{i}}\end{array}$

Pyruvate carboxylase

Pep??? cds??? Pos??? Loc???

Reaction: oxalacetate-Ecoli_1

$$
\begin{array}{rll}
\text { ATP 4.1.1.49 } & \begin{array}{l}
\mathrm{ADP} \\
\mathrm{CO}_{2} \\
\text { Oxalacetic Acid }
\end{array} \\
\text { Phosphoenolpyruvate }
\end{array}
$$

4.1.1.49: Phosphoenolpyruvale Carboxykinase (ATP)

P22259 P13735 P10963

DESCRIPTIONS OF THE ENZYME

desc PHOSPHOENOLfYRUVATE CARBOXYKINASE (ATP)

Alternate name PHOSPHOPYRUVATE CARBOXYLASE (ATP)

Alternate name PHOSPHOENOLPYRUVATE CARBOXYLASE

Alternate name PHOSPHOENOLPYRUVATE CARBOXYKINASE

Activity ATP + OXALOACETATE $=$ ADP + PHOSPHOENOLPYRUVATE $+\mathrm{CO}(2)$. 
P222259 pckA Pos??? 3554737 to 355635.3

\section{DEscriptions of the PEPTIDE}

desC PHOSPHOENOLPYRIIVATE C:ARBOXYKINASE (ATP) (EC: 4.1.1.49)

E.coligene Ir' $\mathrm{KA}$

Reaction: P-enolpyruvate 2

$$
\text { Phosphoenolpyruvate } \stackrel{42.111}{=} 2-\mathrm{P} \text {-Glycerate }
$$

\begin{tabular}{|c|c|c|c|c|}
\hline P0092 & P00925 & P19140 & P06733 & P17182 \\
\hline P04764 & P07322 & P13929 & P21550 & P25704 \\
\hline P1542 & P09104 & P17183 & P07323 & P25696 \\
\hline 15007 & P08324 & P26300 & P26301 & P08734 \\
\hline
\end{tabular}

DESCRIPTIONS OF THE ENZYME

desc ENOLASE

Alternate name PHOSPHOPYRUVATE HYDRATASE

Alternate name 2-PHOSPHOGLYCERATE DEHYDRATASE

Activity 2-PHOSPHO-D-GLYCERATE = PHOSPHOENOLPYRUVATE $+\mathrm{H}(2) \mathrm{O}$.

P08324 eno 59.66 Loc???

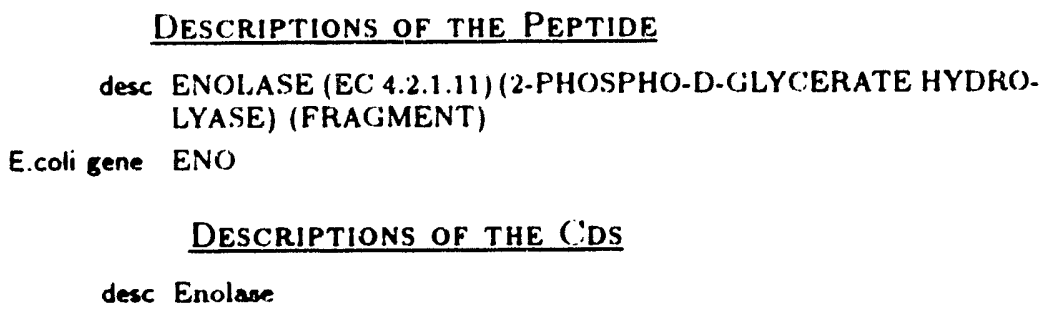

DESCRIPTIONS OF THE PEPTIDE

desC ENOLASE (EC 4.2.1.11) (2.PHOSPHO-D-GLYCERATE HYDRO. LYASE) (FRAGMENT)

E.coli gene ENO

DESCRIPTIONS OF THE (DS

desc Enolase

\begin{tabular}{|c|c|c|}
\hline & 5.4 .2 .1 & \\
\hline 2-P-Gilycerate & 2,3-Diphosphoglycerate & 3-P-Gilycerate \\
\hline
\end{tabular}

Reaction: 2-P-glycerate 2

5.4.2.1: Phosphoglycerale Mutase 


\section{DESCRIPTIONS OF THE ENZYME}

desc PHOSPHOCiLycerate MUTASE

Alternate name PHOSF'HOCILYCERATE PHOSPHOMUTASE

Alternate name PHOSPHOGLYCEROMUTASE

Alternate name P'(iAM

Activity 2-PHOSPHO(iLYCERATE + 2,3-DIPHOSPHOGLYCERATE $=3-$ PHOSPHOGLYCERATE + 2,3-DIPHOSPHOG (BLYCERATE.

Pep??? cds??? Pos??? Loc???

Reaction: 3-P-glycerate_2

$$
\begin{aligned}
\text { ATP } 2.72 .3 & \text { ADP } \\
\text { 3-P-(ilycerate } & 1.3-\text { Diphosphoglycerate }
\end{aligned}
$$

2.7.2.3: Phosphoglycerate Kinase

$\begin{array}{lllll}\text { P00558 } & \text { P09411 } & \text { P07205 } & \text { P09041 } & \text { P 16617 } \\ \text { P25055 } & \text { P08891 } & \text { P08966 } & \text { P07377 } & \text { P08967 } \\ \text { P07378 } & \text { P08892 } & \text { P08893 } & \text { P12782 } & \text { P12783 } \\ \text { P11977 } & \text { P24269 } & \text { P18912 } & \text { P11665 } & \text { P00559 } \\ \text { P14828 } & \text { P20972 } & \text { P20971 } & \text { P09188 } & \text { P09403 } \\ \text { P14228 } & \text { P24590 } & \text { P00560 } & \text { P09404 } & \end{array}$

DESCRIPTIONS OF THE ENZYME

desc P'HOSPHOGLYCERATE KINASE

Activity A'TP + 3-PHOSF'HO-D-GLYCERATE $=$ ADP + 3-PHOSPHOD-(iLYCEROYL PHOSPHATE.

P11665 pgk 63.313089158 from 3090321 complement

\section{DESCRIPTIONS OF THE PEPTIDE}

desc PHOSPHOGLYCERATE KINASE (EC 2.7.2.3)

E.coli gene $\mathrm{P}(\mathrm{iK}$

DESCRIPTIONS OF THE CDS

desc Phosphoglycerate kinase 
Reaction: 1,3-bisPglycerate_2

$$
\begin{aligned}
\text { 1,3-Diphosphoglycerate } & 1.2 .1 .12 \\
\mathrm{NADH}_{2} & \begin{array}{l}
\text { Glyceraldehyde-3-Phosphate } \\
\mathrm{NAD}
\end{array}
\end{aligned}
$$

\begin{tabular}{|c|c|c|c|c|}
\hline 04970 & P07486 & P06977 & P24751 & 100002 \\
\hline 17729 & P00358 & P17329 & P07487 & 1603 \\
\hline 24406 & 17730 & P00359 & 30 & 0360 \\
\hline 17331 & 25856 & 15 & & P09672 \\
\hline & 43 & & 59 & P12860 \\
\hline 44 & P25861 & & 77 & P08735 \\
\hline 17878 & P04796 & P09094 & 97 & 512 \\
\hline P22513 & P20445 & P15115 & 22 & P00362 \\
\hline 209 & P10096 & POC & 48 & P17244 \\
\hline 19089 & P24163 & $49^{\circ}$ & 6 & 357 \\
\hline 24164 & & P1 & 18 & \\
\hline 8 & & & & P2 \\
\hline & P2 & P24753 & P00361 & P17 \\
\hline 09317 & P08439 & P09316 & & \\
\hline
\end{tabular}

1.2.1.12: Glyceraldehyde 3-Phosphate Dehydrogenase

DESCRIPTIONS OF THE ENZYME

desC GLYCERALDEHYDE 3-PHOSPHATE DEHYDROGENASE Alternate name TRIOSEPHOSPHATE DEHYDROGENASE

Alternate name (.APDH

Activity D-\{iLYCERALDEHYDE 3-PHOSPHATE + ORTHOPHOSPHATE + NAD $(+)=3-$ PHOSPHO-D- GLYCEROYL PHOS. PHATE + NADH.

P06977 gapA Pos???? 1873435 to 1874430

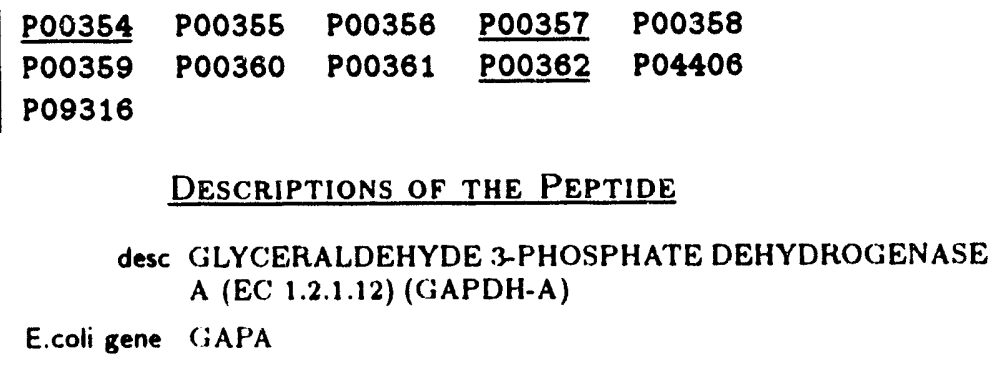

Descriptions OF THE PEPTIDE

desC GLYCERALDEHYDE 3-PHOSPHATE DEHYDROGENASE A (EC 1.2.1.12) (GAPDH-A)

E.coli gene (iAPA

DESCRIPTIONS OF THE (DS 
desc glyceraldehyde 3-phosphate dehydrogenase

P11603 gapB Pos???? 3090371 from 3091390 complement

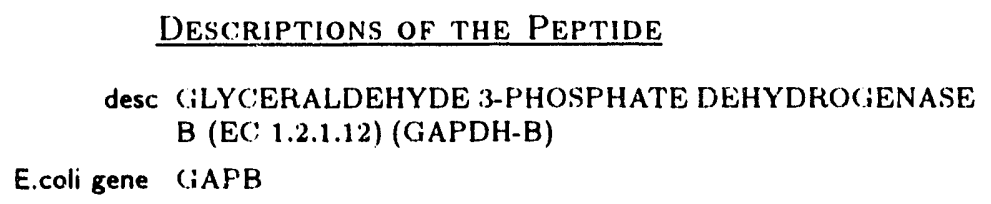

Reaction: glyceraldehyde-3-P_3

$$
\begin{aligned}
& \text { Dihydroxyacetone-Phosphate } \stackrel{4.1 .2 .13}{\rightleftharpoons} \text { Fructose-1,6-P.2 } \\
& \text { Glyceraldehyde-3-Phosphate }
\end{aligned}
$$

4.1.2.13: Fructose-Bisphosphate Aldolase

$\begin{array}{lllll}\mathrm{P} 04075 & \mathrm{P} 05064 & \mathrm{P} 00883 & \mathrm{P} 05065 & \mathrm{P} 07341 \\ \mathrm{P} 05062 & \mathrm{P} 00884 & \mathrm{P} 09972 & \mathrm{P} 05063 & \mathrm{P} 09117 \\ \mathrm{P} 16096 & \mathrm{P} 22197 & \mathrm{P} 19537 & \mathrm{P} 07764 & \mathrm{P} 11604 \\ \mathrm{P} 08440 & \mathrm{P} 17784 & \mathrm{P} 14223 & \mathrm{P} 07752 & \mathrm{P} 14540\end{array}$

DESCRIPTIONS OF THE ENZYME

desc FRUCTOSE-BISPHOSPHATE ALDOLASE

Alternate name ALDOLASE

Alternate name FRUCTOSE-1,6-BISPHOSPHATE TRIOSEPHOSPHATE-LYASE

Activity D-FRUCTOSE 1,6-BISPHOSPHATE = CiLYCERONE PHOSPHATE + D-GLYCERALDEHYDE 3-PHOSPHATE.

P11604 fba 63.27 3087864 from 3088943 complement

Descriptions of the Peptide

desc FRUCTOSE-BISPHOSPHATE ALDOLASE (EC; 4.1.2.13)

E.coli gene FBA

DESCRIPTIONS OF THE CDS

desc fructose-bisphosphate aldolase 
Reaction: glyceraldehyde-3-P_4

Cilyceraldehyde-3-Phosphate $\stackrel{5.11}{=}$ Dihydroxyacetone-Phosphate

5.3.1.1: Triosephosphate lsomerase

$\begin{array}{lllll}\text { P04828 } & \text { P00943 } & \text { P00940 } & \text { P21820 } & \text { P19583 } \\ \text { P04790 } & \text { P00938 } & \text { P00941 } & \text { P15426 } & \text { P12863 } \\ \text { P17751 } & \text { P19118 } & \text { P00939 } & \text { P07669 } & \text { P04789 } \\ \text { P00942 } & & & & \end{array}$

DESCRIPTIONS OF THE ENZYME

desC TRIOSEPHOSPHATE ISOMERASE

Alternate name TRIOSEPHOSPHATE MUTASE

Alternate name PHOSPHOTRIOSE ISOMERASE

Activity D-(iLYC:ERALDEHYDE 3-PHOSPHATE = DIHYDROXY ACETONE PHOSPHATE.

P04790 tpiA 88.684140059 from 4140826 complement

DESCRIPTIONS OF THE PEPTIDE

desc TRIOSEPHOSPHATE ISOMERASE (EC 5.3.1.1) (TIM)

E.coli gene TPIA

DESCRIPTIONS OF THE CDS

desc Triosephosplate isomerase

Reaction: dihydroxyacetone-P.1

Dihydroxyacetone-Phosphate $\stackrel{5.3 .1 .1}{=}$ (ilyceraldehyde-3-Phosphate

5.3.1.1: Triosephosphate Isomerase

\begin{tabular}{lllll} 
P04828 & P00943 & P00940 & P21820 & P19583 \\
P04790 & P00938 & P00941 & P15426 & P12863 \\
P17751 & P19118 & P00939 & P07669 & P04789 \\
\hline
\end{tabular}

DESCRIPTIONS OF THE ENZYME 


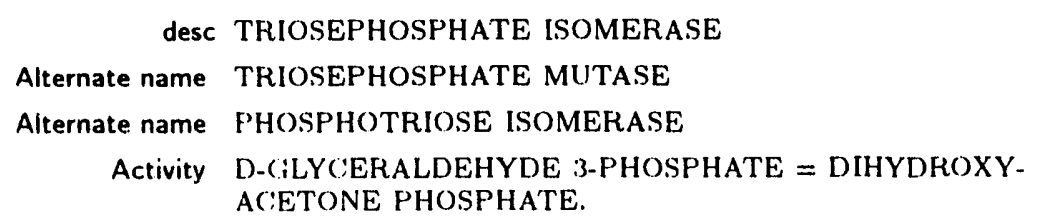

P04790 tpiA 88.684140059 from 4140826 complement

\section{DEscriptions OF THE PEPTIDE}

desc TRIOSEPHOSPHATE ISOMERASE (EC; 5.3.1.1) (TIM)

E.coli gene TPIA

DESCRIP'TIONS OF THE CDS

desc Triosephosphate isomerase

Reaction: fructose-1,6-bisP 1

Fructose-1,6- $\mathrm{P}_{2}$ 3.1.3.11 Fructose-6-P

$\mathrm{H}_{2} \mathrm{O} \rightleftharpoons \mathbf{P}_{\mathrm{i}}$

3.1.3.11: Fructose-Bisphosphatase

$\begin{array}{lllll}\text { P19911 } & \text { P25851 } & \text { P09200 } & \text { P09467 } & \text { P00636 } \\ \text { P00637 } & \text { P19112 } & \text { P09202 } & \text { P09199 } & \text { P22418 } \\ \text { P09195 } & \text { P23014 } & \text { P09201 } & \text { P14766 } & \text { P19912 } \\ \text { P22780 } & & & & \end{array}$

DESCRIPTIONS OF THE ENZYME

desc FRUCTOSE-BISPHOSPHATASE

Alternate name FRUCTOSE-1,6-BISPHOSPHATASE

Alternate name HEXOSEDIPHOSPHATASE

Activity D-FRUCTOSE 1,6-BISPHOSPHATE + H(2)O = D-FRUCTOSE 6-PHOSPHATE + ORTHOPHOSPHATE.

P09200 fbp 96.104485979 from 4486977 complement

Descriptions OF THE PEPTIDE

desc FRUCTOSE-1,6-BISPHOSPHATASE (EC 3.1.3.11) (D-FRUCTOSE1,6-BISPHOSPHATE 1-PHOSPHOHYDROLASE) (FBPASE). 


\section{E.coli gene FBP}

\section{DESCRIPTIONS OF THE (.DS}

desc fructose-bisphosphatase:

Reaction: fructose-6-P 2

$$
\text { Fructose-b-P } \stackrel{5.3 .19}{=} \text { Glucose-6-Phosphate }
$$

5.3.1.9: Glucose-6-Phosphale Isomerase

$\begin{array}{lllll}\text { P13375 } & \text { P13376 } & \text { P11243 } & \text { P11537 } & \text { P06744 } \\ \text { P12341 } & \text { P06745 } & \text { P08059 } & \text { P18240 } & \text { P13377 } \\ \text { P12709 } & & & & \end{array}$

DESCRIPTIONS OF THE ENZYME

desc GLUCOSE-6-PHOSPHATE ISOMERASE

Alternate name PHOSPHOGLUCOSE ISOMERASE

Alternate name PHOSPHOHEXOSE ISOMERASE

Alternate name PHOSPHOHEXOMUTASE

Alternate name OXOISOMERASE

Alternate name HEXOSEPHOSPHATE ISOMERASE

Alternate name PHOSPHOSACCHAROMUTASE

Alternate name PHOSPHOGLUCOISOMERASE

Alternate name PHOSPHOHEXOISOMERASE

Activity D-(iLUCOSE 6-PHOSPHATE = D-FRUCTOSE 6-PHOSPHATE.

P11537.pgi 91.2842633288 to 4264937

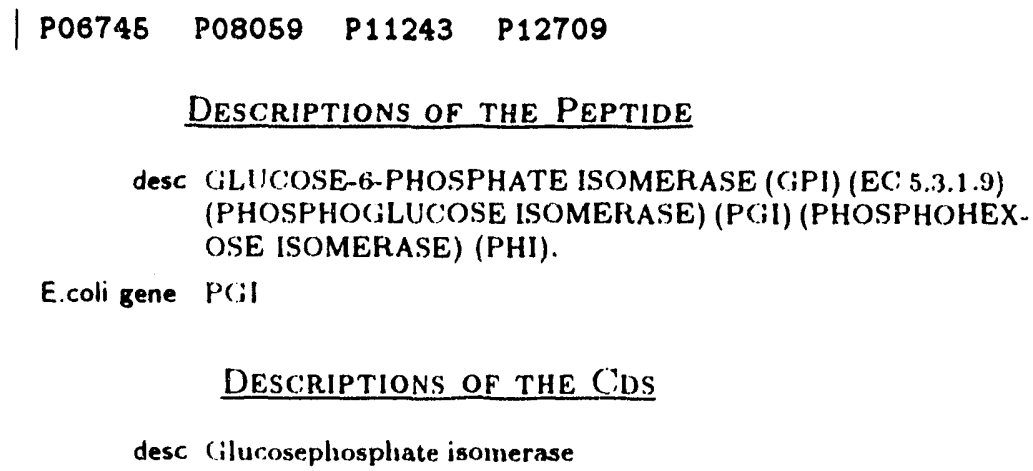

DESCRIPTIONS OF THE CDS

desc (ilucosephosphate isomerase 
Reaction: glucose-6-P_4

$$
\text { Cilucose-6-Phosphate } \stackrel{5.4 .2 .2}{\rightleftharpoons} \text { (ilucose-1,6- } \mathrm{P}_{2}
$$

5.4.2.2: Phosphoglucomutase

$$
\begin{aligned}
& \text { DESCRIPTIONS OF THE ENZYME } \\
& \text { desc PHOSPHOGLUCOMUTASE } \\
& \text { Alternate name } \\
& \text { Activity ALUCOSE PHOSPHOMUTASE } \\
& \text { 6-PHOSPHATE. }
\end{aligned}
$$

Pep? :? cds??? Pos??? Loc???

\begin{tabular}{|c|c|c|}
\hline $\begin{array}{r}\text { ATP } \\
\text { Glucose-1-Phosphate }\end{array}$ & $\stackrel{2.7 .7 .27}{\rightleftharpoons}$ & $\begin{array}{l}\text { ADP-Glucose } \\
\text { PP }_{\mathbf{i}}\end{array}$ \\
\hline
\end{tabular}

Reaction: glucose-1,6-bisP_1

$$
\text { Gilucose-1,6- } \mathrm{P}_{2} \stackrel{5.4 .2 .2}{\rightleftharpoons} \text { Gilucose-1-Phosphate }
$$

5.4.2.2: Phosphoglucomutase

$$
\begin{gathered}
\text { DESCRIPTIONS OF THE ENZYME } \\
\text { desc PHOSPHOGLUCOMUTASE } \\
\text { Alternate name CLUCOSE PHOSPHOMUTASE } \\
\text { Activity ALPHA-D-(iLUCOSE 1-PHOSPHATE = ALPHA-D-CLUUCOSE } \\
\text { 6-PHOSPHATE. } \\
\text { Pep???? c.ds??? Pos??? Loc??? }
\end{gathered}
$$

Reaction: glucose-1-P_2

2.7.7.27: Glucose-1-Phosphate Adenylyltransferase

$\begin{array}{lllll}\text { P15280 } & \text { P23509 } & \text { P12298 } & \text { P12299 } & \text { P12300 } \\ \text { P00584 } & \text { P05415 } & & & \end{array}$

\section{DESCRIPTIONS OF THE ENZYME}




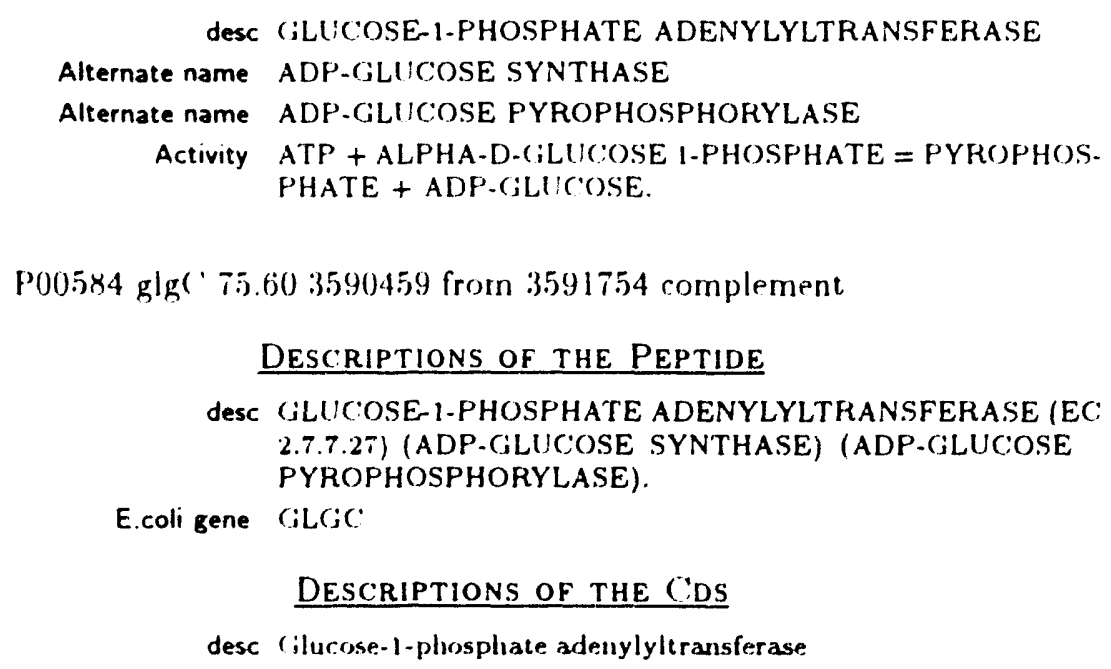

Reaction: ADP-glucose 1

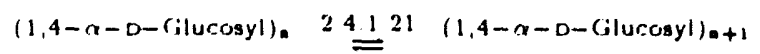

2.4.1.21: Starch (Bacterial Glycogen) Synthase

P08323 P05416

DESCRIPTIONS OF THE ENZYME

desC STARCH (BACTERIAL GLYCOGEN) SYNTHASE

Alternate name (iLYCOOCEN SYNTHASE

Alternate name ADP-(iLUCOSE-STARCH (:LUCOSYLTRANSFERASE

Activity ADP-(iLUCOSE + (1,4-ALPHA-D-(iLUCOSYL)(N) $=$ ADP $+(1,4-A L P H A-D-\operatorname{CLUCOOSYL})(\mathrm{N}+1)$.

P08323 glgA 75.603589026 from 3590459 complement

DEscriptions of THE PEPTIDE

desc GLYCOGEN SYNTHASE (EC 2.4.1.21) (STARCH (BACTERIAL GLYCOGEN) SYNTHASE).

E.coli gene GLCiA

DESCRIPTIONS OF THE CDS

desc Glycogen synthase 


\section{Summary of the Pathway: Glutamate-Synthesis_1}

\begin{tabular}{|rcl|}
\hline $2.00 * 2-$ Ketoglutarate & $\begin{array}{c}1.4 .1 .13 \\
1.4 .1 .4\end{array}$ & ADP \\
$2.00 *$ Ammonium & Glutamate & $2.00 *$ Glutamate \\
ATP & & $\mathrm{Pi}_{i}$ \\
$2.00 * \mathrm{NADPH}_{2}$ & & $\mathrm{H}^{+}$ \\
& & $\mathrm{H}_{2} \mathrm{O}$
\end{tabular}

Reaction: Glutamate-Synthesis_2

$2-$ Ketoglutarate
Ammonium
$\mathrm{NADPH}_{2}$$\stackrel{\text { Glutamate }}{=} \begin{aligned} & \mathrm{NADP} \\ & \mathrm{H}_{2} \mathrm{O}\end{aligned}$

\subsubsection{4: Glutamate Dehydrogenase (NADP $\left.{ }^{+}\right)$

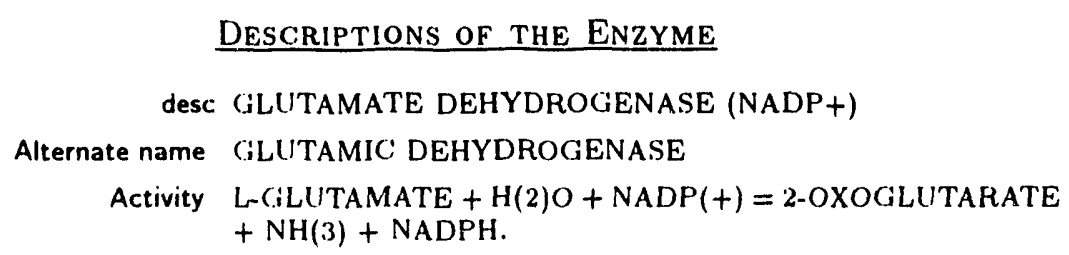

DESCRIPTIONS OF THE ENZYME

desc GLUTAMATE DEHYDROGENASE (NADP+)

Alternate name GLUTAMIC DEHYDROGENASE

Activity L-CiLUTAMATE $+\mathrm{H}(2) \mathrm{O}+\mathrm{NADP}(+)=2$-OXOGLUTARATE $+\mathrm{NH}(3)+\mathrm{NADPH}$.

P00370 gdhA 26.991852934 to 1854277

Descriptions of the Peptide

desc NADP-SPECIFIC GLUTAMATE DEHYDROGENASE (EC: 1.4.1.4)

E.coli gene GDHA

\section{DESCRIPTIONS OF THE CDS}

desc Clutanate dehydrogenase 
Reaction: GLT_1

$$
\begin{array}{rll}
\hline \text { Ammonium } & & \text { ADP } \\
\text { ATP } & \stackrel{3.1 .2}{=} \text { Glutamine } \\
\text { Gilutamate } & & \mathrm{Pi}_{\mathrm{i}} \\
& & \mathrm{H}^{+}
\end{array}
$$

6.3.1.2: Gilutamate-Ammonza Ligase

$\begin{array}{lllll}\text { P05457 } & \text { P20477 } & \text { P14654 } & \text { P07694 } & \text { P04770 } \\ \text { P09826 } & \text { P24099 } & \text { P04772 } & \text { P20478 } & \text { P20805 } \\ \text { P13564 } & \text { P25462 } & \text { P14655 } & \text { P08281 } & \text { P04771 } \\ \text { P22878 } & \text { P19432 } & \text { P14636 } & \text { P14656 } & \text { P08282 } \\ \text { P00965 } & \text { P15102 } & \text { P00964 } & \text { P10583 } & \text { P22248 } \\ \text { P19064 } & \text { P15623 } & \text { P12425 } & \text { P15103 } & \text { P16580 } \\ \text { P10656 } & \text { P04773 } & \text { P11600 } & \text { P06711 } & \text { P15104 } \\ \text { P23712 } & \text { P04078 } & \text { P15124 } & \text { P21154 } & \text { P15105 } \\ \text { P25821 } & \text { P12424 } & \text { P20479 } & \text { P09606 } & \text { P13499 } \\ \text { P06201 } & \text { P15106 } & \text { P23794 } & \text { P07804 } & \text { P19904 }\end{array}$

DESCRIPTIONS OF THE ENZYME

desc GLUTAMATE-AMMONIA LIGASE

Alternate name GLUTAMINE SYNTHETASE

Activity ATP + L-GLUTAMATE + NH(3) = ADP + ORTHOPHOSPHATE + LGILUTAMINE.

P06711 glnA 87.484086048 from 4087460 complement

DEscriptions OF THE PEPTIDE

desc (;LUTAMINE SYNTHETASE (EC 6.3.1.2) (GLUTAMATEAMMONIA LIGASE)

E.coli gene (iLNA

DESCRIPTIONS OF THE CDS

desc Glutamine synthetase

Reaction: 2-Ketoglutarate 2

$$
\begin{array}{r}
2-\text { Ketoglutarate } \\
\text { Glutamine } \\
\mathrm{NADPH}_{2}
\end{array} \quad \stackrel{\text { NADP }}{=} \begin{aligned}
& 2.00 * \text { Glutamate } \\
& \text { NADP }
\end{aligned}
$$

1.4.1.13: Glutamate Synthase (NADPH)

P09831 P09832 


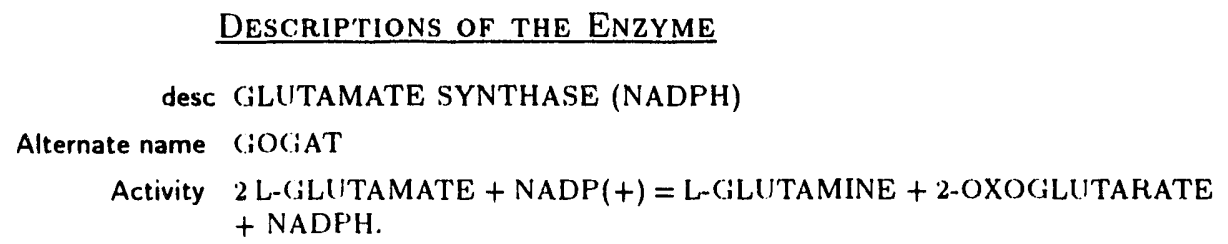

P09831 gltB 69.8233373367 to 3377911

\section{Descriptions of the Peptide}

desc GLUTAMATE SYNTHASE (NADPH) LARGE CHAIN PRECURSOR (EC 1.4.1.13)

E.coli gene GLTB

\section{DESCRIPTIONS OF THE ('DS}

desc glutamate synthase; large subunit

P09832 gltD 69.823377924 to 3379339

DESCRIPTIONS OF THE PEPTIDE

desc (ILUTAMATE SYNTHASE (NADPH) SMALL CHAIN (EC 1.4.1.13)

E.coli gene CILTD

DESCRIPTIONS OF THE CDS

desc glutamate synthase; small subunit 


\section{Summary of the Pathway: Glycolysis_1}

\begin{tabular}{|c|c|c|}
\hline $2.00 \backsim \mathrm{ADP}$ & $\begin{array}{c}1.2 .1 .12 \\
2.7 .1 .11 \\
2.7 .1 .2 \\
2.7 .1 .40 \\
2.7 .2 .3 \\
2.7 .5 .3 \\
4.1 .2 .13 \\
4.21 .11 \\
53.11 \\
3.3 .1\end{array}$ & $2.00=\mathrm{ATP}$ \\
\hline (ilucose & $2.00 \backsim A T P$ & $2.00-\mathrm{NADH}_{2}$ \\
\hline $2.00 * \mathrm{NAD}$ & & 2.00 Pyruvate \\
\hline $2.00 * \mathrm{P}_{\mathrm{i}}$ & & $2.00 * \mathrm{H}_{2} \mathrm{O}$ \\
\hline
\end{tabular}

Reaction: glucose_1

$$
\begin{aligned}
\text { ATP } 2.7 .1 .2 & \begin{array}{l}
\text { ADP } \\
\text { Gilucose }
\end{array} \\
& \begin{array}{l}
\text { Glucose-6-Phosphate } \\
\mathrm{H}^{+}
\end{array}
\end{aligned}
$$

2.7.1.2: Glucokinase

DESCRIPTIONS OF THE ENZYME

desc CLUUCOKINASE

Activity ATP + D-GLUCOSE $=$ ADP + D-GLUCOSE 6-PHOSPHATE.

Pep??? glk 51.78 Loc???

\section{DESCRIPTIONS OF THE C.DS}

desc (ilucokinase

Reaction: glucose-6-P_3

$$
\text { Cilucose-6-Phosphate } \stackrel{5.3 .1 .9}{=} \text { Fructose }-6-P
$$

5.3.1.9: Glucose-6-Phosphate Isomerase

$\begin{array}{lllll}\text { P13375 } & \text { P13376 } & \text { P11243 } & \text { P11537 } & \text { P06744 } \\ \text { P12341 } & \text { P06745 } & \text { P08059 } & \text { P18240 } & \text { P13377 } \\ \text { P12709 } & & & & \end{array}$




\section{DESCRIPTIONS OF THE ENZYME}

desc (iLUCOSE-6-PHOSPHATE ISOMERASE

Alternate name PHOSPHOGLUCOSE ISOMERASE

Alternate name PHOSPHOHEXOSE ISOMERASE

Alternate name PHOSPHOHEXOMUTASE

Alternate name OX()ISOMERASE

Alternate name HEXOSEPHOSPHATE ISOMERASE

Alternate name PHOSPHOSACCHAROMUTASE

Alternate name PHOSPHOGLUCOISOMERASE

Alternate name PHOSPHOHEXOISOMERASE

Activity D-GLUCOSE 6-PHOSPHATE = D-FRUCTOSE 6-PHOSPHATE.

Pl15.37 pgi 91.284263288 to 4264937

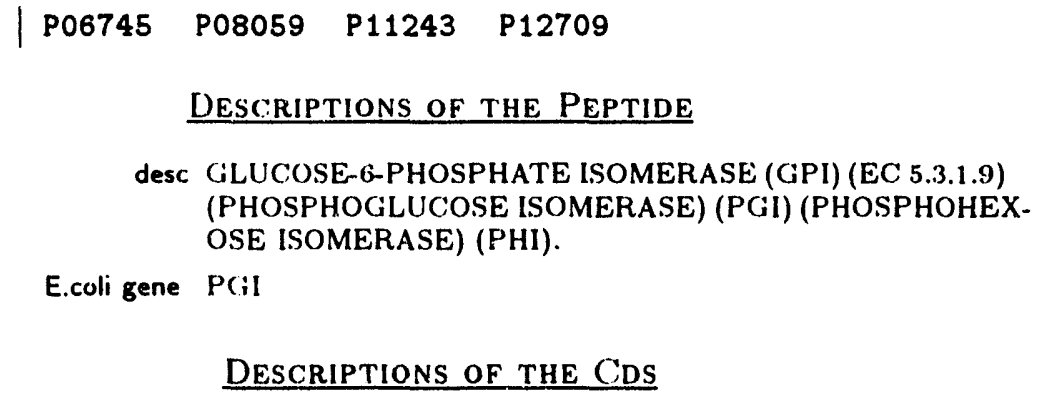

\section{DESCRIPTIONS OF THE CDS}

desc Glucosephosphate isomerase

Reaction: fructose-6-P_1

$$
\begin{array}{rll}
\text { ATP } 2.7 .1 .11 & \begin{array}{l}
\text { ADP } \\
\text { Fructose-6-P }
\end{array} & \begin{array}{l}
\text { Fructose-1,6- } P_{2} \\
\mathrm{H}^{+}
\end{array}
\end{array}
$$

2.7.1.11: 6-Phosphofructokinase

\begin{tabular}{|c|c|c|c|c|}
\hline P06998 & P80019 & P21777 & P16861 & P06999 \\
\hline P21778 & P16862 & P00512 & P08237 & P12382 \\
\hline P00511 & P20275 & P17858 & & \\
\hline \multicolumn{5}{|c|}{ DESCRIPTIONS OF THE ENZYME } \\
\hline & $A C$ & HOSPH & Cr & ASE \\
\hline Alter & name & OSPHOI & KOKIN & \\
\hline
\end{tabular}




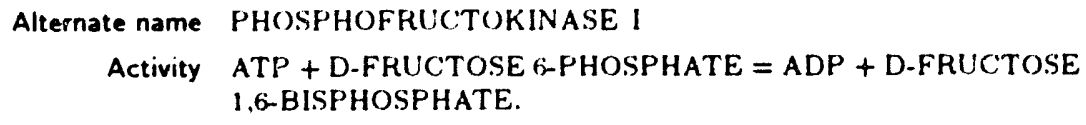

DESCRIPTIONS OF THE PEPTIDE

desc 6-PHOSPHOFRUCTOKINASE ISOZYME I (EC: 2.7.1.11) (PHOSPHOFRUCTOKINASE-1)

E.coli gene PFKA

DESCRIPTIONS OF THE C.DS

desc 6-Phosphofructokinase I

P06999 pfkB 37.741814595 to 1815521

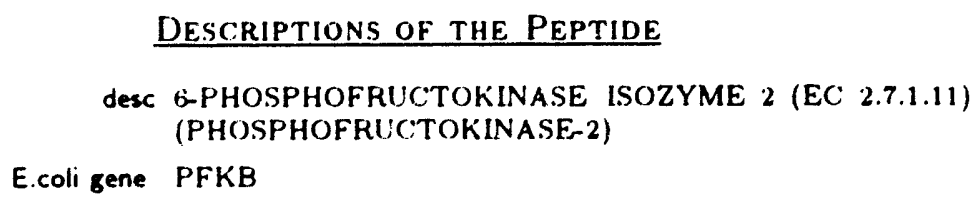

DESCRIPTIONS OF THE CDS

desc Level of 6-phosphofructokinase II; suppressor of pikA

Reaction: fructose-1,6-bisP 2

$$
\text { Fructose }-1.6-P_{2} \stackrel{4.12 .13}{=} \begin{aligned}
& \text { Dihydroxyacetone-Phosphate } \\
& \text { Cilyceraidehyde-3-Phosphate }
\end{aligned}
$$

4.1.2.13: Fruclose-Bisphosphale Aldolase

\begin{tabular}{lllll} 
P04075 & P05064 & P00883 & P05065 & P07341 \\
\hline P05062 & P00884 & P09972 & P05063 & P09117 \\
P16096 & P22197 & P19537 & P07764 & P11604 \\
P08440 & P17784 & P14223 & P07752 & P14540
\end{tabular}

DESCRIPTIONS OF THE ENZYME

desc FRIIC:TOSE-BISPHOSPHATE ALDOLASE 


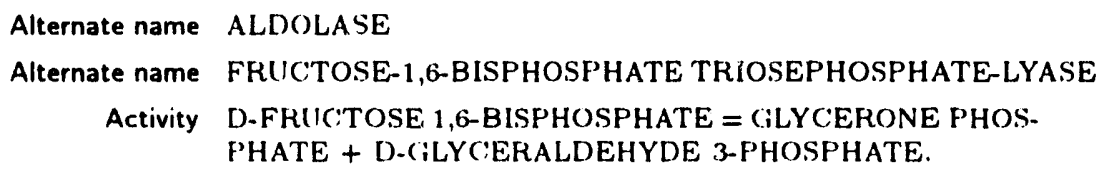

P11604 fba 63.273087864 from 3088943 complement

DESCRIPTIONS OF THE PEPTIDE

desc FRUCTOSE-BISPHOSPHATE ALDOLASE (EC 4.1.2.13)

E.coli gene FBA

\section{DESCRIPTIONS OF THE CDS}

desc fructose-bisphosphate aldolase

Reaction: dihydroxyacetone-P_1

Dihydroxyacetone-Phosphate $\stackrel{5.3 .1}{=}$ Glyceraldehyde-3-Phosphate

5.3.1.1: Triosephosphate Isomerase

$\begin{array}{lllll}\mathrm{P} 04828 & \mathrm{P} 00943 & \mathrm{P} 00940 & \mathrm{P} 21820 & \mathrm{P} 19583 \\ \mathrm{P} 04790 & \mathrm{P} 00938 & \mathrm{P} 00941 & \mathrm{P} 15426 & \mathrm{P} 12863 \\ \mathrm{P} 17751 & \mathrm{P} 19118 & \mathrm{P} 00939 & \mathrm{P} 07669 & \underline{\mathrm{P} 04789} \\ \mathrm{P} 00942 & & & & \end{array}$

DESCRIPTIONS OF THE ENZYME

desc TRIOSEPHOSPHATE ISOMERASE

Alternate name TRIOSEPHOSPHATE MUTASE

Alternate name PHOSPHOTRIOSE ISOMERASE

Activity D-GLYCERALDEHYDE 3-PHOSPHATE = DIHYDROXYAC:ETONE PHOSPHATE.

P04790 tpiA 88.684140059 from 4140826 complement

DESCRIPTIONS OF THE PEPTIDE

desc TRIOSEPHOSPHATE ISOMERASE (EC 5.3.1.1) (TIM)

E.coli gene TPIA 


\section{DESCRIPTIONS OF THE CDS}

desc Triosephosphate isomerase

Reaction: glyceraldehyde-3-P 1

$$
\begin{aligned}
& \text { Glyceraldehyde-3-Phosphate } 1.2 .1 .12 \quad 1,3-D i p h o s p h o g l y c e r a t e \\
& P_{i} \\
& \mathrm{NADH}_{2}
\end{aligned}
$$

\begin{tabular}{|c|c|c|c|c|}
\hline 0 & 486 & P06977 & P24751 & \\
\hline 9 & 00358 & P17329 & P07487 & \\
\hline 06 & 17730 & 00359 & 7330 & \\
\hline 7331 & 25856 & 09315 & 12858 & 96 \\
\hline 19866 & P09043 & P25857 & 12859 & 286 \\
\hline 9044 & 25861 & P25858 & P08477 & 0873 \\
\hline 17878 & 04796 & P09094 & P10097 & P2251 \\
\hline 22513 & P20445 & P15115 & 22 & $\mathrm{P} 0036$ \\
\hline 09124 & 10096 & P00356 & 748 & 24 \\
\hline 19089 & P24163 & P24749 & P2 & P0038 \\
\hline 64 & 19 & P1 & 18 & $P 1$ \\
\hline 16 & P00355 & P20286 & PO & P24 \\
\hline 20287 & P24166 & P24753 & P00361 & P1772 \\
\hline 09317 & P08439 & P09316 & & \\
\hline
\end{tabular}

1.2.1.12: Glyceraldehyde 3-Phosphate Dehydrogenase

\section{DESCRIPTIONS OF THE ENZYME}

desc GLYCERALDEHYDE 3-PHOSPHATE DEHYDROGENASE Alternate name TRIOSEPHOSPHATE DEHYDROGENASE

Alternate name $\mathrm{GAPDH}$

Activity D-GLYCERALDEHYDE 3-PHOSPHATE + ORTHOPHOSPHATE + NAD $(+)=3$-PHOSPHO-D-GLYCEROYL PHOSPHATE + NADH.

P06977 gapA Pos??? 1873435 to 1874430

\begin{tabular}{|lllll} 
P00354 & P00355 & P00356 & P00357 & P00358 \\
P00359 & P00360 & P00361 & P00362 & P04406 \\
P09316 & & & &
\end{tabular}

DEscriptions of THE PEPTIDE 


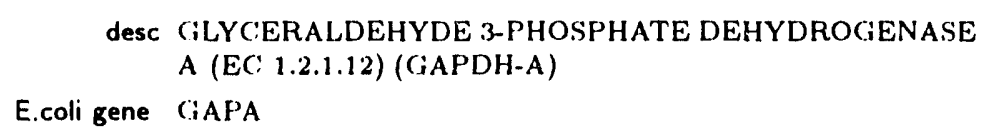

DESCRIPTIONS OF THE CDS

desc glyceraldehyde 3-phosphate dehydrogenase

Pl1603 gap B Pos??? 3090371 from 3091390 complement

\section{DESCRIPTIONS OF THE PEPTIDE}

desc (ILYCERALDE YDE 3-PHOSPHATE DEHYDROCIENASE B (EC: 1.2.1.12) (GAPDH-B)

E.coligene (iAPB

Reaction: 1,3-bisPglycerate 1

\begin{tabular}{|c|c|c|}
\hline $\begin{array}{r}\text { ADP } \\
\text { 1,3-Diphosphoglycerate }\end{array}$ & $\stackrel{2.7 .2 .3}{=}$ & $\begin{array}{l}\text { ATP } \\
3-P \text {-Glycerate }\end{array}$ \\
\hline
\end{tabular}

2.7.2.3: Phosphoglycerale Kinase

$\begin{array}{lllll}\text { P00558 } & \text { P09411 } & \text { P07205 } & \text { P09041 } & \text { P } 16617 \\ \text { P25055 } & \text { P08891 } & \text { P08966 } & \text { P07377 } & \text { P08967 } \\ \text { P07378 } & \text { P08892 } & \text { P08893 } & \text { P12782 } & \text { P 12783 } \\ \text { P11977 } & \text { P24269 } & \text { P18912 } & \text { P11665 } & \text { P } 00559 \\ \text { P14828 } & \text { P20972 } & \text { P } 20971 & \text { P09188 } & \text { P09403 } \\ \text { P14228 } & \text { P24590 } & \text { P00560 } & \text { P09404 } & \end{array}$

DESCRIPTIONS OF THE ENZYME

desc PHOSPhoglilycerate kinase

Activity ATP + 3-PHOSPHO-D-GLYCERATE $=$ ADP + 3-PHOSPHOD-GLYCEROYL PHOSPHATE.

P11665 pgk 63.31 3089158 from 3090321 complement

\section{DESCRIPTIONS OF THE PEPTIDE}

desc PHOSPHOGL ' CERATE KINASE (EC 2.7.2.3)

E.coli gene $\mathrm{P}(\mathrm{iK}$ 


\section{DESCRIPTIONS OF THE CDS}

desc Phosphoglycerate kinase

Reaction: 3-P-glycerate_1

$$
3-\mathrm{P}-\text { Glycerate } \stackrel{2.7 .5 .3}{\rightleftharpoons} 2-\mathrm{P}-\text { Glycerate }
$$

2.7.5.3: Transferred Entry: 5.4.\$.1

DESCRIPTIONS OF THE ENZYME

desc TRANSFERRED ENTRY: 5.4.2.1

Pep??? cds??? Pos??? Loc???

\section{Reaction: 2-P-glycerate_.1}

$$
\text { 2-P-Cilycerate } \stackrel{4.2 .1 .11}{=} \begin{aligned}
& \text { Phosphoenolpyruvate } \\
& \mathrm{H}_{2} \mathrm{O}
\end{aligned}
$$

$\begin{array}{lllll}\text { 4.2.1.11: Enolase } & & & \\ \text { P00924 } & \text { P } 00925 & \text { P } 19140 & \text { P } 06733 & \text { P } 17182 \\ \text { P04764 } & \text { P } 07322 & \text { P } 13929 & \text { P } 21550 & \text { P } 25704 \\ \text { P15429 } & \text { P } 09104 & \text { P17183 } & \text { P } 07323 & \text { P } 25696 \\ \text { P15007 } & \text { P08324 } & \text { P } 26300 & \text { P } 26301 & \text { P08734 }\end{array}$

DESCRIPTIONS OF THE ENZYME

desc ENOLASE

Alternate name PHOSPHOPYRUVATE HYDRATASE

Alternate name 2-PHOSPHOGLYCERATE DEHYDRATASE

Activity 2-PHOSPHO-D-GLYCERATE = PHOSPHOENOLPYRUVATE $+\mathrm{H}(2) \mathrm{O}$.

P08324 eno 59.66 Loc???

\section{DESCRIPTIONS OF THE PEPTIDE}

desc ENOLASE (EC 4.2.1.11) (2-PHOSPHO-D-GLYCERATE HYDROLYASE) (FRAGMENT) 


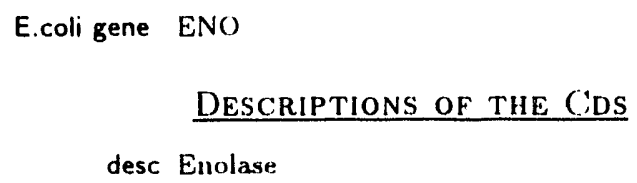

Reaction: P-enolpyruvate 1

$$
\begin{array}{rrl}
\text { ADP } & 2.7 .1 .40 & \text { ATP } \\
\text { Phosphoenolpyruvate } & \rightleftharpoons & \text { Pyruvate } \\
\mathrm{H}^{+} & &
\end{array}
$$

2.7.1.40: Pyruvale Kinase

$\begin{array}{lllll}\text { P14178 } & \text { P11979 } & \text { P14618 } & \text { P11974 } & \text { P11980 } \\ \text { P19680 } & \text { P21599 } & \text { P14786 } & \text { P11981 } & \text { P22200 } \\ \text { P22360 } & \text { P00548 } & \text { P00549 } & \text { P11973 } & \text { P04763 } \\ \text { P12928 } & & & & \end{array}$

\section{DESCRIPTIONS OF THE ENZYME}

desc PYRUVATE KINASE

Alternate name PHOSPHOENOLPYRUVATE KINASE

Alternate name PHOSPHOENOL TRANSPHOSPHORYLASE

Activity ATP + PYRUIVATE = ADP + PHOSPHOENOLPYRUVATE.

P14178 pykF 36.531764989 to 1766377

DESCRIPTIONS OF THE PEPTIDE

desc PYRUVATE kINASE I (EC: 2.7.1.40) (PK-1)

E.coli gene PYKF

\section{DESCRIPTIONS OF THE (DS}

desc Pyruvate kinase $\mathrm{F}$

P21599 pykA Pos??? 1948303 to 1949745

Descriptions of the Peptide

desc PYRUVATE KINASE II (EC 2.7.1.40) (PK-2)

E.coli gene PYKA 


\section{Summary of the Pathway: Histidine-Synthesis_1}

\begin{tabular}{|c|c|c|}
\hline $\begin{array}{r}\text { ATP } \\
\text { (ilutamine } \\
2.00 * N A D \\
\alpha-D-5-\text { Phosphoribosylpp } \\
3.00 * \mathrm{H}_{2} \mathrm{O}\end{array}$ & $\begin{array}{c}1.1 .1 .23 \\
2.4 .2 .17 \\
2.6 .1 .9 \\
3.1 .3 .15 \\
3.5 .4 .19 \\
3.6 .1 .31 \\
1.2 .1 .19 \\
5.3 .1 .16 \\
\text { A midocycla.e } \\
\mathrm{H}^{+} \\
2.0 \mathrm{O} \mathrm{H}_{2} \mathrm{O} \\
\rightleftharpoons\end{array}$ & $\begin{array}{l}\text { 2-Ketoglutarate } \\
\mathrm{Z}-\text { Nucleotide } \\
\text { Histidine } \\
2.00 * \mathrm{NADH}_{2} \\
\mathrm{Pi}_{\mathrm{i}} \\
2.00 * \mathrm{PP}_{\mathrm{i}} \\
2.00 * \mathrm{H}^{+}\end{array}$ \\
\hline
\end{tabular}

\section{Reaction: PRPP_HIS_1}

\begin{tabular}{|c|c|c|}
\hline $\begin{array}{r}\text { ATP } \\
H^{+} \\
\alpha-D-5-\text { Phosphoribosylpp }\end{array}$ & $\stackrel{2.4 .2 .17}{\rightleftharpoons}$ & $\begin{array}{l}\text { Phosphoribosyl-ATP } \\
\text { PP }_{i}\end{array}$ \\
\hline
\end{tabular}

\subsubsection{7: ATP Phosphoribosyltransferase}

$\begin{array}{llll}\text { P10366 P05148 P00499 P00498 } & \text { P }\end{array}$

\section{DESCRIPTIONS OF THE ENZYME}

desc ATP PHOSPHORIBOSYLTRANSFERASE

Alternate name PHOSPHORIBOSYL-ATP PYROPHOSPHORYLASE

Activity 1-(5-PHOSPHO-D-RIBOSYL)-ATP + PYROPHOSPHATE $=A T P+5-P H O S P H O-A L P H A-D-R I B O S E$ 1-DIPHOSPHATE.

P10366 hisG 44.002102220 to 2103119

DESCRIPTIONS OF THE PEPTIDE

desc ATP PHOSPHORIBOSYLTRANSFERASE (EC 2.4.2.17)

E.coli gene HISC:

DESCRIPTIONS OF THE CDS

desc ATF phosphoribosyltransferase 
Reaction: Phosphoribosyl-ATP_HIS_1

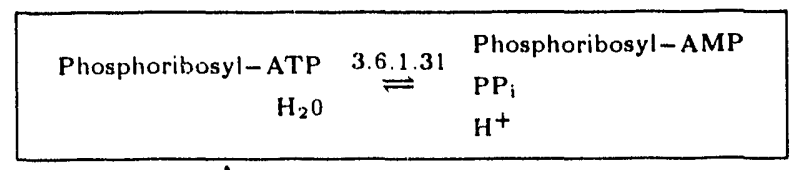

3.6.1.31: Phosphoribosyl-ATP Pyrophosphatase.

$\begin{array}{llll}\text { P06989 P07685 P10367 P00815 } & \text { P }\end{array}$

DESCRIPTIONS OF THE ENZYME

desc PHOSPHORIBOSYL-ATP PYROPHOSPHATASE

Activity 5-PHOSPHORIBOSYL-ATP $+\mathrm{H}(2) \mathrm{O}=5$ - $\mathrm{PHOSPHORIBOSYL-}$ AMP + PYROPHOSPHATE.

P06989 hisl 44.012108642 to 2109253

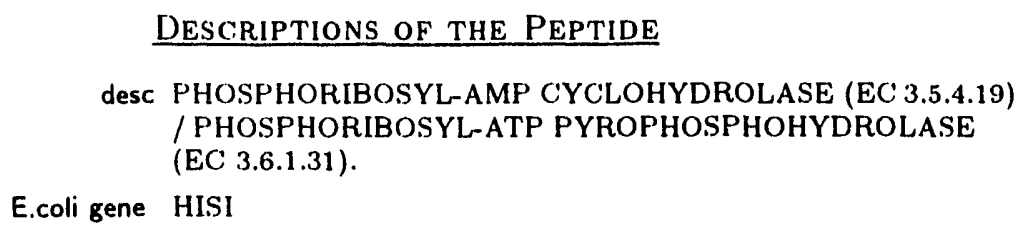

DESCRIPTIONS OF THE PEPTIDE

desC PHOSPHORIBOSYL-AMP CYCLOHYDROLASE (EC 3.5.4.19) / PHOSPHORIBOSYL-ATP PYROPHOSPHOHYDROLASE (EC 3.6.1.31).

E.coli gene HISI

\section{DESCRIPTIONS OF THE CDS}

desc phosphoribosyl-AMP cyclohydrolase; phosphoribosyl-ATP pyrophosphatase

\section{Reaction: Phosphoribosyl-AMP_HIS_1}

Phosphoribosyl-AMP 3.5.4.19 Phosphoribosyl Formimino-AlCAR $\mathrm{H}_{2} \mathrm{O} \rightleftharpoons \mathrm{H}^{+}$

\subsubsection{9: Phosphoribosyl-AMP Cyclohydrolase}

$\begin{array}{llll}\mathrm{P} 06989 & \mathrm{P} 07685 & \mathrm{P} 10367 \quad \mathrm{P} 00815\end{array}$

DESCRIPTIONS OF THE ENZYME

desc PHOSPHORIBOSYLAMP CYCLOHYDROLASE

Activity 5-PHOSPHORIBOSYL-AMP $+\mathrm{H}(2) \mathrm{O}=$ 5-(5-PHOSPHO-

D-RIBOSYLAMINOFORMIMINO)- 1-(5-PHOSPHO-RIBOSYL) IMIDAZOLE-4-C:ARBOXAMIDE. 
P06989 hisl $44.01210 \times 642$ to 2109253

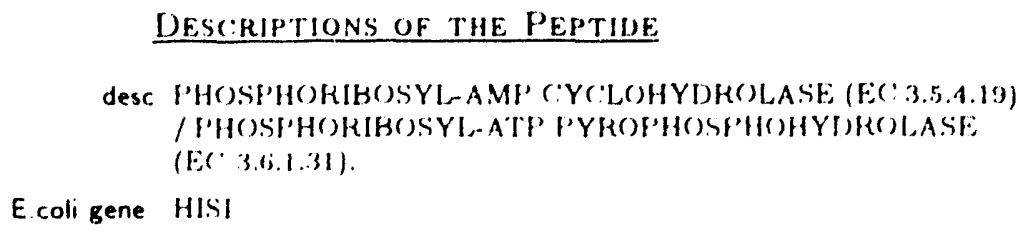

\section{DESCRIPTIONS OF THE CDS}

desc phosplooribonyl-AMP cyclohydrolase; phossphoribonyl-ATP pyrophosphatase

Reaction: Phosphoribosyl Formimino-5-AI-4-Carboxamide_Ribotide_HIS_1

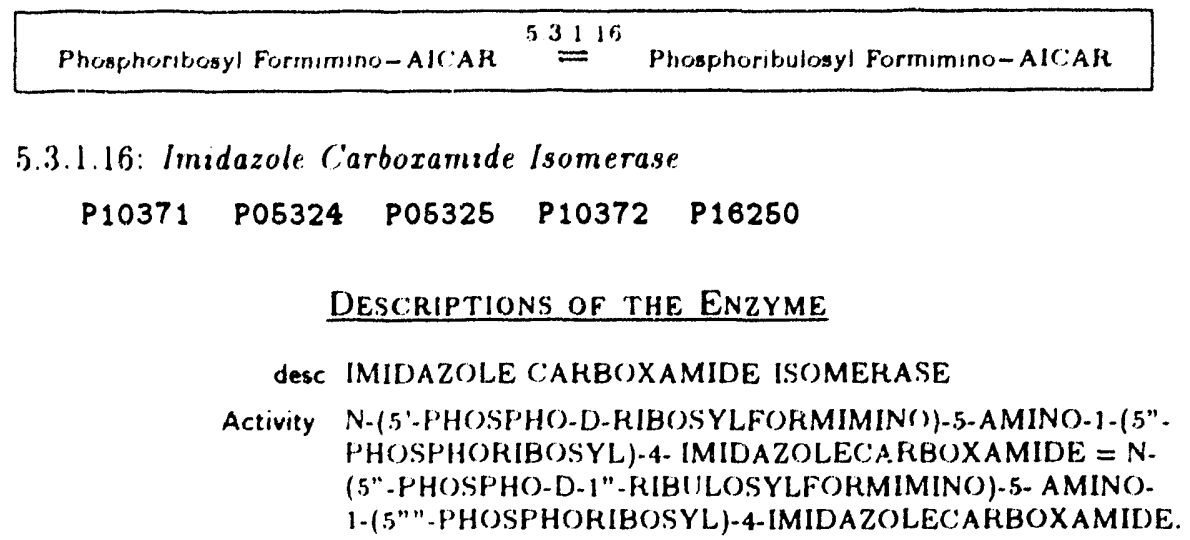

P10371 hisA 44.012107153 to 2107890

DESCRIPTIONS OF THE PEPTIDE

desc PHOSPHORIBOSYLFORMIMINO-5-AMINOIMIDAZOLE CARBC)XAMIDE RIBOTIDE ISOMERASE (EC 5.3.1.16).

E.coli gene HISA

\section{DESCRIPTIONS OF THE CDS}

desc N-(5'-phospho-L-ribosylformimino)-5-amino-1-(5'-phosphoribosyl)4-imidazolecartooxanide isomerase 
Reaction: Phosphoribulosyl_Formimino-5-AI-4-Carboxamide_Ribotide_HIS_1

$\begin{aligned} & \text { (ilutamine Amidocyclase } \begin{array}{l}Z \text {-Nucleotıde } \\ \mathrm{D} \text {-Erythro-Imidazole-Glycerol-P } \\ \text { Phosphoribulosyl Formimino-AIC:AR }\end{array} \\ & \text { Cilutamate }\end{aligned}$

Amidocyclase

Pep??? cds???? Pos??? Loc????

Reaction: D-Erythro-Imidazole-Glycerol-P_HIS_1

$$
\begin{array}{|lll|}
\hline \text { D-Erythro-Imidazole-Glycerol-P } \stackrel{4.2 .1 .19}{\rightleftharpoons} \begin{array}{l}
\text { Imidazole-Acetol-P } \\
\mathrm{H}_{2} \mathrm{O}
\end{array} \\
\hline
\end{array}
$$

4.2.1.19: Imidazoleglycerol-Phosphate Dehydratase

$$
\begin{aligned}
& \begin{array}{lllll}
\text { P18787 } & \text { P06987 } & \text { P10368 } & \text { P16247 } & \text { P06633 }
\end{array} \\
& \text { DESCRIPTIONS OF THE ENZYME } \\
& \text { desC IMIDAZOLEGLYCEROL-PHOSPHATE DEHYDRATASE } \\
& \text { Activity D-ERYTHRO-1-(IMIDAZOL-4-YL)GLYCEROL 3-PHOSPHATE } \\
& \text { = 3-(IMIDAZOL-4-YL)- 2-OXOPROPYL PHOSPHATE + } \\
& \mathrm{H}(2) \mathrm{O} \text {. }
\end{aligned}
$$

P06987 hisB 44.002105496 to 2106563

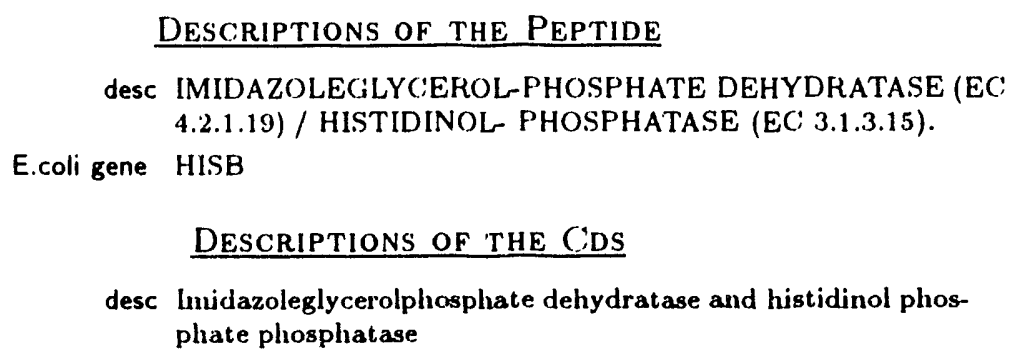

Reaction: Imidazole-Acetol-P_HIS_1

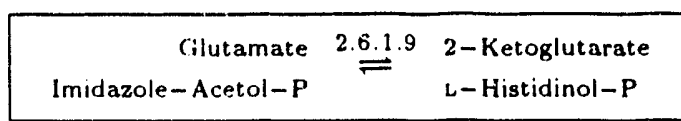

2.6.1.9: Histidinol-Phosphate Aminotransferase 
$\begin{array}{lllll}\text { P17731 } & \text { P06986 } & \text { P17736 } & \text { P10369 } & \text { P16246 } \\ \text { P07172 } & & & \end{array}$

\section{DESCRIPTIONS OF THE ENZYME}

desc HISTIDINOL-PHOSPHATE AMINOTRANSFERASE

Alternate name IMIDAZOLYLACETOLPHOSPHATE AMINOTRANSFERASE

Activity L-HISTIDINOL-PHOSPHATE + 2-OXOGLUTARATE $=3-$ (IMIDAZOL-4-YL)-2-OXOPROPYL PHOSPHATE + L-(ILUTAMATE.

P06986 his(: 44.002104426 to 2105496

\section{DESCRIPTIONS OF THE PEPTIDE}

desc HISTIDINOL-PHOSPHATE AMINOTRANSFERASE (EC 2.6.1.9)

E.coli gene HISC

\section{DESCRIPTIONS OF THE CDS}

desc Histidinol phosphate aninotransferase

\section{Reaction: L-Histidinol-P_HIS_1}

L-Histidinol-P 3.1.3.15 Histidinol

$\mathrm{H}_{2} \mathrm{O} \quad \mathrm{P}_{\mathrm{i}}$

3.1.3.15: Histidinol-Phosphatase

P06987P10368

DESCRIPTIONS OF THE ENZYME

desC HISTIDINOL-PHOSPHATASE

Activity L-HISTIDINOLPHOSPHATE $+\mathrm{H}(2) \mathrm{O}=$ L-HISTIDINOL + ORTHOPHOSPHATE.

P06987 hisB 44.002105496 to 2106563

DESCRIPTIONS OF THE PEPTIDE

desC IMIDAZOLEGLYCEROL-PHOSPHATE DEHYDRATASE (EC 4.2.1.19) / HISTIDINOL PHOSPHATASE (EC 3.1.3.15).

E.coli gene HISB 


\section{DESCRIP'TIONS OF THE CDS}

desc Inidazoleglycerolphosphate dehydratase and histidinol phosphate phosphatase

Reaction: Histidinol_HIS_1

$$
\begin{array}{rll}
\text { Histidinol } & 1.1 .1 .23 & \begin{array}{l}
\text { Histidine } \\
2.00 * \mathrm{NAD}
\end{array} \\
\mathrm{H}_{2} \mathrm{O} & & \begin{array}{l}
2.00 * \mathrm{NADH}_{2} \\
\mathrm{H}^{+}
\end{array}
\end{array}
$$

1.1.1.23: Histidinol Dehydrogenase.

$\begin{array}{lllll}\text { P07685 } & \text { P00815 } & \text { P18786 } & \text { P24226 } & \text { P06988 } \\ \text { P10370 } & \text { P16245 } & & & \end{array}$

DESCRIPTIONS OF THE ENZYME

desc HISTIDINOL DEHYDROGENASE

Activity L-HISTIDINOL $+2 \mathrm{NAD}(+)=$ L-HISTIDINE $+2 \mathrm{NADH}$.

P06988 hisD 44.002103125 to 2104429

DESCRIPTIONS OF THE PEPTIDE

desc HISTIDINOL DEHYDROGENASE (EC: 1.1.1.23) $(\mathrm{HDH})$

E.coli gene HISD

DESCRIPTIONS OF THE CDS

desc L-Histidinol:NAD+ oxidoreductase 


\section{Summary of the Pathway: Homoserine-Synthesis_1}

\begin{tabular}{rcl|}
\hline ATP & 1.1 .1 .3 & ADP \\
L-Aspartate & 2.7 .2 .4 & Homoserine \\
$2.00 * \mathrm{NADPH}_{2}$ & & $2.00 *$ NADP \\
& & $\mathrm{P}_{\mathrm{i}}$ \\
\hline
\end{tabular}

\section{Reaction: L-Aspartate_1}

\begin{tabular}{rl|}
$\mathrm{ATP}$ & 2.7 .2 .4 \\
$\mathrm{~L}$-Aspartate & ADP \\
Aspartate-4-P
\end{tabular}

2.7.2.4: Aspartate Kinase

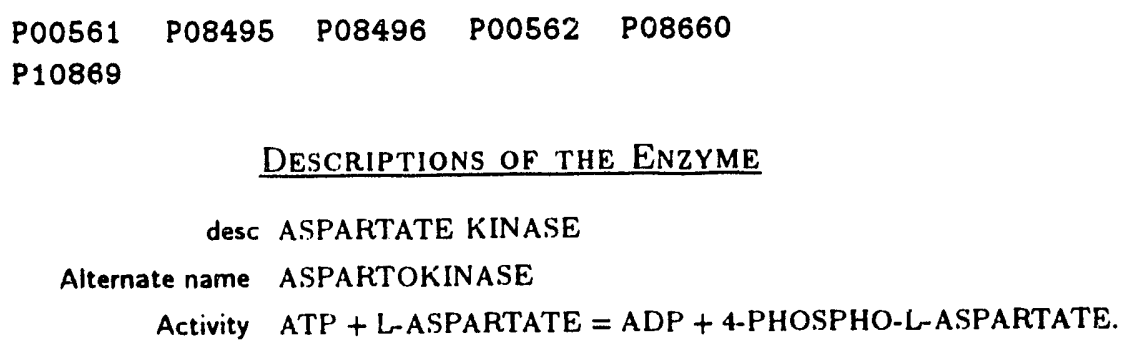

DESCRIPTIONS OF THE ENZYME

desc ASPARTATE KINASE

Aiternate name ASPARTOKINASE

Activity ATP + L-ASPARTATE $=$ ADP + 4-PHOSPHO-L-ASPARTATE.

P00561 thrA 0.00407 to 2869

\section{DESCRIPTIONS OF THE PEPTIDE}

desc ASPARTOKINASE I (EC: 2.7 .2 .4$)$ / HOMOSERINE DEHYDROGENASE I (EC; 1.1.1.3).

E.coli gene THRA

\section{DESCRIPTIONS OF THE C'DS}

desc aspartokinase I-homoserine dehydrogenase I

P00562 metL 88.994158997 to 4161429

\section{DESCRIPTIONS OF THE PEPTIDE}

desc ASPARTOKINASE II (EC; 2.7.2.4) / HOMOSERINE DEHYDROGENASE II (EC 1.1.1.3). 
E.coli gene METL

DESCRIPTIONS OF THE C.DS

desc aspartokinase II ; homoserine dehydrogenase II

P03660 lys(: $91.244261+14$ from 4262763 complement

Descriptions of the Peptide

desc LYSINE-SENSITIVE ASPARTOKINASE III (EC: 2.7.2.4)

E.coli gene LYSC:

DESCRIPTIONS OF THE CDS

desc aspartokinase [II

Reaction: L-beta-Aspartyl-P_1

Aspartate-4-P 1.2.1.11 L-Aspartate-Semialdehyde

$\mathrm{NADPH}$ NADP

$P_{i}$

1.2.1.11: Aspartate-Semialdehyde Dehydrogenase

$\begin{array}{llll}\text { P00353 P10539 P23247 } & \text { P13663 }\end{array}$

DESCRIPTIONS OF THE ENIZYME

desc ASPARTATE-SEMIALDEHYDE DEHYDROGENASE

Activity L-ASPARTATE-SEMIALDEHYDE + ORTHOPHOSPHATE

+ NADP $(+)=$ L-ASPARTYL PHOSPHATE + NADPH.

P00353 asd 75.703596204 from 3597307 complement

DESCRIPTIONS OF THE PEPTIDE

desc ASPARTATE-SEMIALDEHYDE DEHYDROGENASE (EC; 1.2.1.11)

E.coligene ASD

DESCRIPTIONS OF THE CDS 
desc aspartate semialdehyde dehydrogenase

Reaction: L-Aspartate-Semialdehyde 2

L-Aspartate-Semialdehyde $\stackrel{1.1 .33}{=} \begin{aligned} & \text { Homoserine } \\ & \mathrm{NADPH}_{2}\end{aligned}$
NADP

1.1.1.3: Homoserine Dehydrogenase

$\begin{array}{llll}\text { P00561 P00562 P19582 } & \text { P08499 }\end{array}$

DESCRIPTIONS OF THE ENZYME

desc HOMOSERINE DEHYDROGENASE

Activity L-HOMOSERINE + NAD $(P)(+)=$ L-ASPARTATE BETASEMIALDEHYDE + NAD (P)H.

P00561 thrA 0.00407 to 2869

DESCRIPTIONS OF THE PEPTIDE

desc ASPARTOKINASE I (EC 2.7.2.4) / HOMOSERINE DEHYDROGENASE I (EC 1.1.1.3).

E.coli gene THRA

DESCRIPTIONS OF THE C.DS

desc aspartokinase I-homoserine dehydrogenase I

P00562 metL 88.994158997 to 4161429

DESCRIPTIONS OF THE PEPTIDE

desc ASPAKTOKINASE II (EC 2.7.2.4) / HOMOSERINE DEHYDROGENASE II (EC 1.1.1.3).

E.coli gene METL

DESCRIPTIONS OF THE CDS

desc aspartokinase II ; homoserine dehydrogenase II 


\section{Summary of the Pathway: IMP-Synthesis_1}

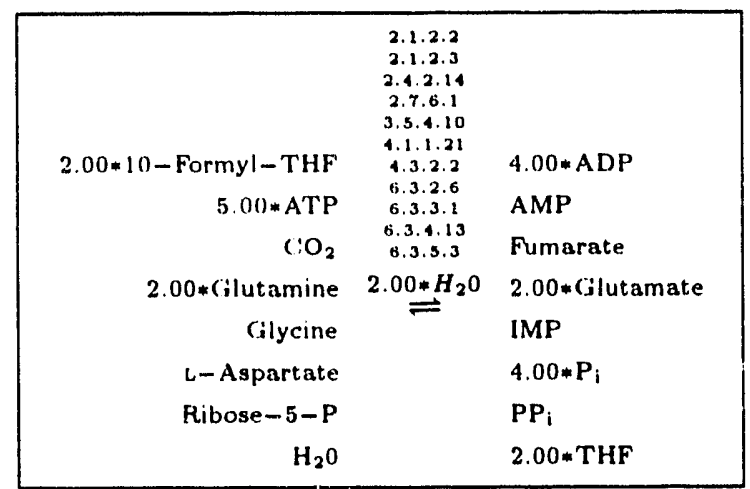

Reaction: Ribose-5P_Purine_1

$$
\begin{aligned}
& \text { ATP } 2.7 .6 .1 \\
& \text { Ribose-5-P } \text { AMP } \\
& \alpha-D-5-\text { Phosphoribosylpp }
\end{aligned}
$$

2.7.6.1: Ribose-Phosphate Pyrophosphokinase

$\begin{array}{lllll}\text { P09329 } & \text { P11908 } & \text { P09330 } & \text { P21108 } & \text { P14193 } \\ \text { P08330 } & \text { P15849 } & & & \end{array}$

DESCRIPTIONS OF THE ENZYME

desc RIBOSE-PHOSPHATE PYROPHOSPHOKINASE

Alternate name PHOSPHORIBOSYL PYROPHOSPHATE SYNTHETASE

Activity ATP + D-RIBOSE 5-PHOSPHATE = AMP + 5-PHOSPHOALPHA-D-RIBOSE 1-DIPHOSPHATE.

P08330 prs 26.491268223 from 1269170 complement

\section{DEscriptions OF THE PEPTIDE}

desc RIBOSE-PHOSPHATE PYROPHOSPHOKINASE (EC 2.7.6.1) (PHOSPHORIBOSYL PYROPHOSPHATE SYNTHETASE).

E.coli gene PRS

\section{DESCRIPTIONS OF THE CDS}

desc Phosphoribosylpyrophosphate synthetase 
Reaction: PRPP_Purine_1

\begin{tabular}{|c|c|c|}
\hline $\begin{array}{r}\text { Cilutamine } \\
\alpha-D-5-\text { Phosphoribosyipp }\end{array}$ & $2 \stackrel{4.2 .14}{=}$ & $\begin{array}{l}5-P-\beta-D-\text { Ribosyl-Amine } \\
\text { Gilutamate }\end{array}$ \\
\hline $\mathrm{H}_{2} \mathrm{O}$ & & $\mathbf{P P}_{\mathbf{i}}$ \\
\hline
\end{tabular}

2.4.2.14: Amidophosphoribosyliransferase.

P00497 P00496 P04046

DESCRIPTIONS OF THE ENZYME

desC AMIDOPHOSPHORIBOSYLTRANSFERASE

Alternate name CLUTAMINE PHOSPHORIBOSYLPYROPHOSPHATE AMIDOTRANSFERASE

Alternate name PHOSPHORIBOSYLDIPHOSPHATE 5-AMIDOTRANSFERASE

Activity 5-PHOSPHO-BETA-D-RIBOSYLAMINE + PYROPHOSPHATE

+ L-GiLUTAMATE $=$ L-GLUTAMINE + 5-PHOSPHO-ALPHA-

D-RIBOSE 1-DIPHOSPHATE + H(2)O.

P00496 purF 50.002446890 from 2448407 complement

\section{Descriptions of the Peptide}

desc AMIDOPHOSPHORIBOSYLTRANSFERASE (EC 2.4.2.14) (GLUTAMINE PHOSPHORIBOSYLPYROPHOSPHATE AMIDOTRANSFERASE).

E.coli gene PURF

\section{DESCRIPTIONS OF THE CDS}

desc anidophosphoribosyl trasisferase

Reaction: 5-P-Ribosyl-Amine_Purine_1

$$
\begin{aligned}
& \text { 5-P- } \beta-D-R i b o s y l-A m i n e \quad 6.3 .4 .13 \quad 5^{\prime}-P-R i b o s y l-C i l y c i n a m i d e \\
& \text { ATP } \stackrel{6.3 .4 .13}{=} \mathrm{ADP} \\
& \text { Glycine } \quad P_{i}
\end{aligned}
$$

6.3.4.13: Phosphoribosylamine-Glycine Ligase

$\begin{array}{lllll}\text { P12039 } & \text { P21872 } & \text { P00967 } & \text { P16340 } & \text { P15640 } \\ \text { P22102 } & \text { P20772 } & \text { P07244 } & & \end{array}$


DESCRIPTIONS OF THE ENZYME

desc PHOSPHORIBOSYLAMINE-GLYCINE LIGASE

Alternate name PHOSPHORIBOSYLCILYCINAMIDE SYNTHETASE

Alternate name (ILYCINAMIDE RIBONUC:LEOTIDE SYNTHETASE

Alternate name (iARS

Activity ATF + 5-PHOSPHORIBOSYLAMINE + GLYCINE = ADP + ORTHOPHOSPHATE + 5"-PHOSPHORIBOSYLC.LYCINAMIDE.

P15640 purD 90.504234826 from 4236115 complement

\section{DESCRIPTIONS OF THE PEPTIDE}

desc PHOSPHORIBOSYLAMINE-GLYCINE LIGASE (EC: 6.3.4.13)

(( AARS) (CILYCINAMIDE RIBONUCLEOTIDE SYNTHETASE) (PHOSPHORIBOSYLGILYCINAMIDE SYNTHETASE).

E.coli gene PURD

DESCRIPTIONS OF THE CDS

desc phosphoribosylglycineamide synthetase

Reaction: 5-P-Ribosyl-Glycineamide_Purine_1

\begin{tabular}{|c|c|c|}
\hline $\begin{array}{r}\mid 0-\text { Formyl-THF } \\
5^{\prime}-\mathrm{P}-\text { Ribosyl-Cilycinamide }\end{array}$ & $\stackrel{2.1 .2 .2}{\rightleftharpoons}$ & FGAR \\
\hline
\end{tabular}

2.1.2.2: Phosphoribosylglycinamide Formyllransferase
P21872
P00967
P16340
P22102 P12040
P08179 P04161

\section{DESCRIPTIONS OF THE ENZYME}

desc PHOSPHORIBOSYLGLYCINAMIDE FORMYLTRANSFERASE

Alternate narne 5'-PHOSPHORIBOSYLGLYCINAMIDE TRANSFORMYLASE

Alternate name GAR TRANSFORMYLASE

Activity 10-FORMYLTETRAHYDROFOLATE + 5'-PHOSPHORIBOSYLGLYCINAMIDE = TETRAHYDROFOLATE + 5"-PHOSPHORIBOSYLNFORMYLGLYCINAMIDE.

P08179 purN 533.80 2630216 to 2630854 


\section{DESCRIPTIONS OF THE PEPTIDE \\ desc PHOSPHORIBOSYLGLYCINAMIDE FORMYLTRANSFERASE (EC: 2.1.2.2) (GART) (GAIL TRANSFORMYLASE) 5'-PHOSPIORIBOSYLGLYCINAMIIDE 'TRANSFORMYLASE). E.coli gene P'URN}

\section{DESCRIPTIONS OF THE (DS}

desc 5 '-phosphoribosyglycinanide transformylase

\section{Reaction: FGAR_Purine_1}

\begin{tabular}{rll|}
\hline ATP & 5-Phosphoribosyl- $N$-Formylglycinearnidine \\
FCiAR & 6.3.5.3 & $\mathrm{ADP}$ \\
Glutamine & Cilutamate \\
$\mathrm{H}_{2} \mathrm{O}$ & $\mathrm{Pi}_{\mathrm{i}}$ \\
\hline
\end{tabular}

\subsubsection{3: Phosphoribosylformylglycinamidine Synthase}

\section{P12041 P12042 P15254}

\section{DESCRIPTIONS OF THE ENZYME}

desc PHOSPHORIBOSYLFORMYLGLYCINAMIDINE SYNTHASE Alternate name PHOSPHORIBOSYLFORMYLGLYCINAMIDINE SYNTHETASE Alternate name FC:AM SYNTHASE

Activity ATP + 5'-PHOSPHORIBOSYLFORMYLGLYCINAMIDE + L- (ILUTAMINE + H(2)O = ADP + ORTHOPHOSPHATE $+5 "$-PHOSPHORIBOSYLFORMYLGLYCINAMIDINE + LCiLUTAMATE.

P15254 purL 55.20 2701613 from 2705500 complement

\section{DESCRIPTIONS OF THE PEPTIDE}

desc PHOSPHORIBOSYLFORMYLGLYCINAMIDINE SYNTHASE (EC 6.3.5.3) (FGAM SYNTHASE).

E.coli gene PURL

\section{DESCRIPTIONS OF THE CDS}

desc phosphoribosylfonnylglycineamide synthetase ; homologous to pur(i of S. typhimurium 


\section{Reaction: FGAM_Purine_1}

$$
\begin{aligned}
& \text { 5-Phosphoribosyl-N-Formylglycineamidine } \stackrel{6.3 .3 .1}{=} \begin{array}{l}
5^{\prime} \text {-Phosphoribosyl-5-Aminoimidazole } \\
\text { ADP }
\end{array} \\
& \text { ATP } \\
& \mathbf{P}_{\mathbf{i}}
\end{aligned}
$$

6.3.3.1: Phosphorivosylformylglyc.namidine Cyclo-Ligase

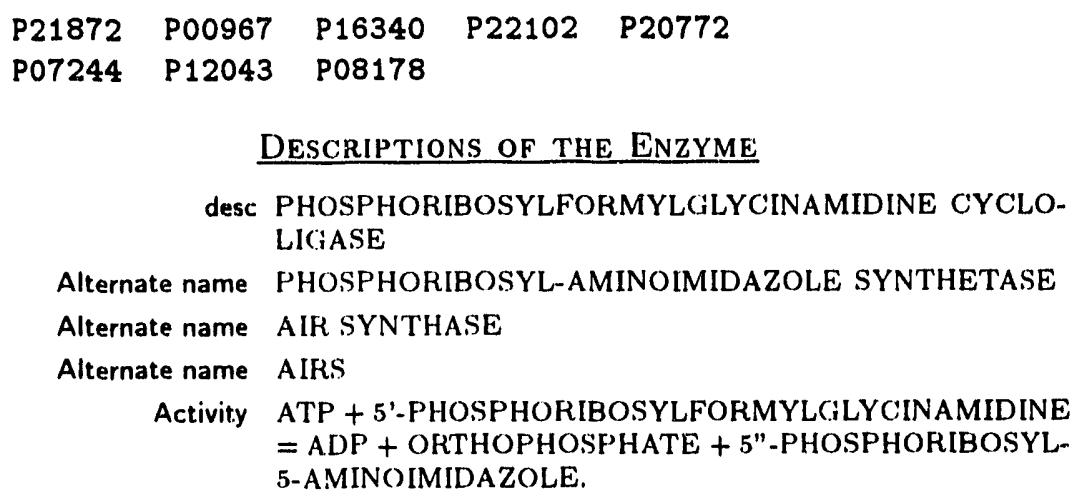

DESCRIPTIONS OF THE ENZYME

desc PHOSPHORIBOSYLFORMYLGLYCINAMIDINE CYCLOLICiASE

Alternate name PHOSPHORIBOSYL-AMINOIMIDAZOLE SYNTHETASE

Alternate name AIR SYNTHASE

Alternate name AIRS

Activity ATP +5 '-PHOSPHORIBOSYLFORMYLGLYCINAMIDINE $=$ ADP + ORTHOPHOSPHATE + 5"-PHOSPHORIBOSYL5-AMINOIMIDAZOLE.

P08178 purM 53.802629179 to 2630216

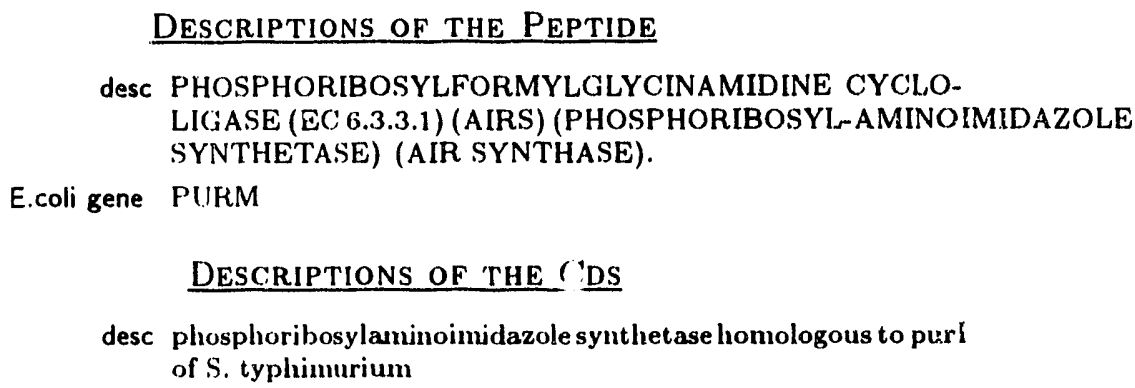

DESCRIPTIONS OF THE PEPTIDE

desc PHOSPHORIBOSYLFORMYLGLYCINAMIDINE CYCLOLIGASE (EC 6.3.3.1) (AIRS) (PHOSPHORIBOSYL-AMINOIMIDAZOLE SYNTHETASE) (AIR SYNTHASE).

Reaction: 5'-P-ribosyl-5-AI_Purine.1

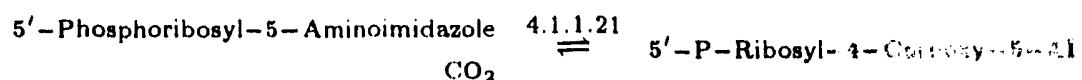

4.1.1.21: Phosphoribosylaminoimidazole Carboxylase.

$\begin{array}{lllll}\text { P12044 } & \text { P09028 } & \text { P22234 } & \text { P15567 } & \text { P21264 } \\ \text { P12045 } & \text { P09029 } & & & \end{array}$




\section{DESCRIPTIONS OF THE ENZYME}

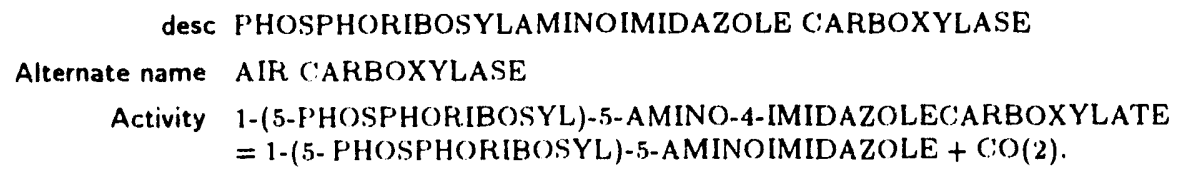

P09028 purE 11.61558926 from 559435 complement

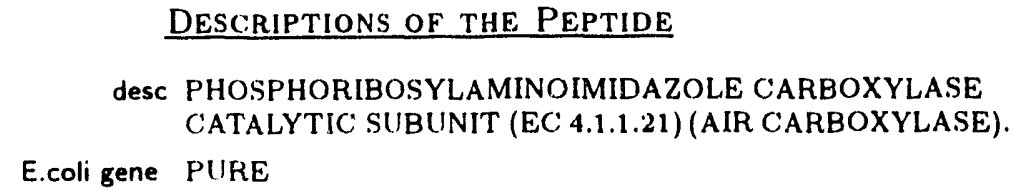

desc phosphoribosylaninoinudazole carboxylase catalytic subunit

P09029 purK 12.19557862 from 558929 complement

\section{DESCRIPTIONS OF THE PEPTIDE}

desC PHOSPHORIBOSYLAMINOIMIDAZOLE C:ARBOXYLASE CO(2) FIXATION SUBUINIT (EC; 4.1.1.21) (AIR CARBOXYLASE).

E.coli gene PURK

\section{DESCRIPTIONS OF THE CDS}

desc phosphoribosylatninoinudazole carboxylase; $\mathrm{CO}_{2}$-fixing subunit

\section{Reaction: 5-P-ribosyl-4-Carboxy-5-AI_Purine_1}

$$
\begin{aligned}
& \text { 5'-P-Ribosyl-4-Carboxy-5-Al } \left.6.3 .2 .6 \quad 5^{\prime}-\mathrm{P}-\text { Ribosyl-4-(N-Succinocarboxamide }\right)-5-\mathrm{Al} \\
& \mathrm{ATP} \stackrel{\text { ADP }}{\rightleftharpoons} \\
& \text { L-Aspartate } \quad P_{i}
\end{aligned}
$$

6.3.2.6: Phosphoribosylaminoimidazole-Succinocarboxamide Synthase

P22234 P12046 P21155 


\section{DESCRIPTIONS OF THE ENZYME}

desc PHOSPHORIBOSYLAMINOIMIDAZOLE-SUCCINOCARBOXAMIDE SYNTHASE

Activity ATF' + 1-(5-PHOSPHORIBOSYL)-4-CARBOXY-5-AMINOIMIDAZOLE + L-ASPARTATE = ADP + ORTHOPHOSPHATE + 1-(5PHOSPHORIBOSYL)-4-(N-SUCCINO-CARBOXAMIDE)- 5AMINOIMIDAZOLE.

P21155 pur(: 53.192605331 from 2606044 complement.

Descriptions of the Peptide

desC PHOSPHORIBOSYLAMINOIMIDAZOLE-SUCCINOCARBOXAMIDE SYNTHASE (EC; 6.3.2.6) (SAICAR SYNTHETASE).

E.coli gene PIIRC

DESCRIPTIONS OF THE C.DS

desc phosphoribosylaminoinidazole-succinocarboxanidesynthetase

Reaction: 5'-p-ribosyl-4-succinocarboxamide-5-AI_Purine_1

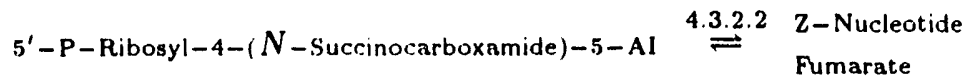

4.3.2.2: Adenylosuccinale Lyase.

\section{DESCRIPTIONS OF THE ENZYME}

desc ADENYLOSUC:CINATE LYASE

Alternate name ADENYLOSUCCINASE

Activity ADENYLOSUCCINATE = FUMARATE + AMP.

Pep??? purB 25.42 Loc???

DESCRIPTIONS OF THE CDS

desc adenylosuccinate lyase 
Reaction: AICAR_Purine_1

10-Formyl-THF 2.1.2.3 5-Phosphoribosyl-5-Formamido-4-Imidazole Carboxamide Z-Nucleotide $=\mathrm{THF}$

2.1.2.3: Phosphoribosylaminoimidazolecarboxamide Formyltransferase

P12048 P15639

-

DESCRIPTIONS OF THE ENZYME

desc PHOSPHORIBOSYLAMINOIMIDAZOLECARBOXAMIDE FORMYLTRANSFERASE

Alternate name AICAR TRANSFORMYLASE

Activity 10-FORMYLTETRAHYDROFOLATE + 5'-PHOSPHORIBOSYL5-AMIN()-4- IMIDAZOLECAARBOXAMIDE = TETRAHYDROFOLATE + 5"-PHOSPHORIBOSYL-5- FORMAMIDO4-IMIDAZOLECAARBOXAMIDE.

P15639 purH 90.504236127 from 4237716 complement

DEScRIPTIONS OF THE PEPTIDE

desc PHOSPHORIBOSYLAMINOIMIDAZOLECARBOXAMIDE FORMYLTRANSFERASE (EC 2.1.2.3) (AICAR TRANSFORMYLASE) / IMP CYCLOHYDROLASE (EC! 3.5.4.10).

E.coligene PURH

DESCRIPTIONS OF THE CDS

desc phosphoribosylaminoimidazolecarboxamide formyltransferase

Reaction: 5-P-Ribosyl-5-Formamido-4-Imid-Carboxamide_Purine_1

$$
\text { 5-Phosphoribosyl-5-Formamido-4-Imidazole Carboxamide } \stackrel{3.54 .10}{\rightleftharpoons} \begin{aligned}
& \rightleftharpoons \mathrm{MP} \\
& \mathrm{H}_{2} \mathrm{O}
\end{aligned}
$$

\subsubsection{0: IMP Cyclohydrolase}

P12048 P15639

DESCRIPTIONS OF THE ENZYME 
desc IMP CYYCLOHYDROLASE

Activity IMP $+\mathrm{H}(2) \mathrm{O}=$ 5-FORMAMIDO-1-(5-PHOSPHORIBOSYL)IMIDAZOLE4. CARBOXAMIDE.

P15639 purH 90.504236127 from 4237716 complement

DESCRIPTIONS OF THE PEPTIDE

desc PHOSPHORIBOSYLAMINOIMIDAZOLECARBOXAMIDE FORMYLTRANSFERASE (EC, 2.1.2.3) (AICAR TRANSFORMYLASE) / IMP CYCLOHYDROLASE (EC 3.5.4.10).

E.coli gene PURH

\section{DESCRIPTIONS OF THE CDS}

desc phosphoribosylanuinoinudazolecarboxanide formyltransferase 


\section{Summary of the Pathway: Isoleucine-Synthesis_1}

\begin{tabular}{|ccl|}
\hline & \multicolumn{1}{c|}{$\begin{array}{c}2.1 .86 \\
1.1 .42 \\
4.1 .18\end{array}$} & \\
& 4.1 .16 & $2-$ Ketoglutarate \\
Cilutamate & Pyridoxal Phosphate & Ainmonium \\
$\mathrm{NADPH}_{2}$ & $\mathrm{H}_{2} \mathrm{O}$ & $\mathrm{CO}_{2}$ \\
Pyruvate & & Iso-Leucine \\
Threonine & & $\mathrm{NADP}$ \\
& & $\mathrm{H}_{2} \mathrm{O}$
\end{tabular}

Reaction: THR_ILE_1

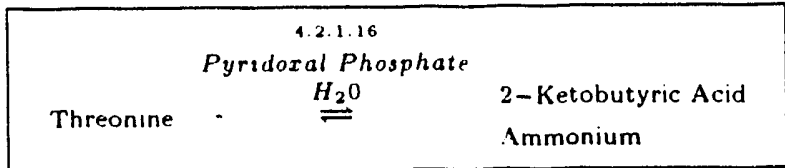

\subsubsection{6: Threonine Dehydratase

$\begin{array}{lllll}\text { P25379 } & \text { P04968 } & \text { P25306 } & \text { P20506 } & \text { P05792 } \\ \text { P11954 } & \text { P00927 } & & & \end{array}$

DESCRIPTIONS OF THE ENZYME

desc THREONINE DEHYDRATASE

Alternate name THREONINE DEAMINASE

Alternate name L-SERINE DEHYDRATASE

Alternate name SERINE DEAMINASE

Activity L-THREONINE $+\mathrm{H}(2) \mathrm{O}=2$-OXOBUTANOATE $+\mathrm{NH}(3)$ $+\mathrm{H}(2) \mathrm{O}$.

P04968 ilvA 84.913983239 to 3984783

DESCRIPTIONS OF THE PEPTIDE

desc THREONINE DEHYDRATASE BIOSYNTHETIC (EC 4.2.1.16) (THREONINE DEAMINASE).

E.coli gene ILVA

DESCRIPTIONS OF THE CDS

desc threonine deaminase 
P05792 tdcB Pos???? 3284565 from 3285554 complement

\author{
DESCRIPTIONS OF THE PEPTIDE \\ desc THREONINE DEHYDRATASE CATABOLIC: (EC 4.2.1.16) \\ (THREONINE DEAMINASE) \\ E.coli gene TDCB
}

\title{
Reaction: 2-Ketobutyric_acid_ILE_1
}

$$
\begin{aligned}
& 2-\text { Ketobutyric Acid } \stackrel{4.1 .3 .18}{\rightleftharpoons} \begin{array}{l}
2-\text { Aceto-2-Hydroxy-Butyrate } \\
\text { Pyruvate }
\end{array} \\
& \mathrm{CO}_{2}
\end{aligned}
$$

4.1.3.18: Acetolactate Synthase

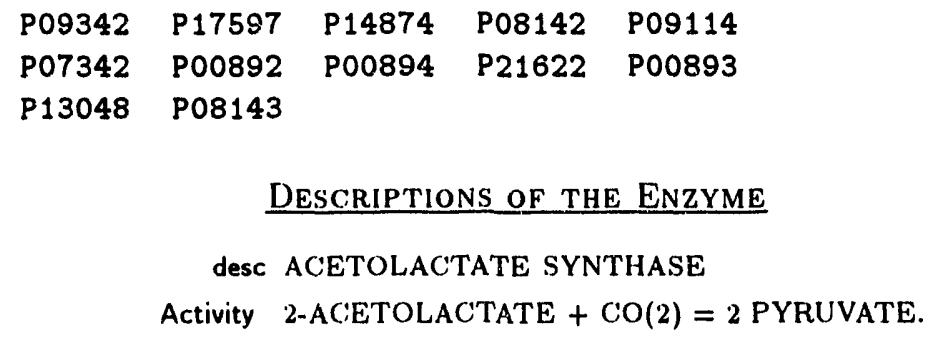

DESCRIPTIONS OF THE ENZYME

desc ACETOLACTATE SYNTHASE

Activity 2-ACETOLACTATE + $\mathrm{CO}(2)=2$ PYRUVATE.

P00892 ilv(i 84.903978469 to 3980115

Descriptions of the Peptide

desc ACETOLACTATE SYNTHASE (EC 4.1.3.18) ISOZYME II, LARGE SUBUNIT (A.HAS- II) (ACETOHYDROXY-ACID SYNTHASE (1).

E.coli gene ILVG.

\section{DESCRIPTIONS OF THE CDS}

desc Acetohydroxy acid synthase II ; valine-insensitive large subunit

P00893 ilvl 1.8385897 to 87618

\section{DESCRIPTIONS OF THE PEPTIDE}




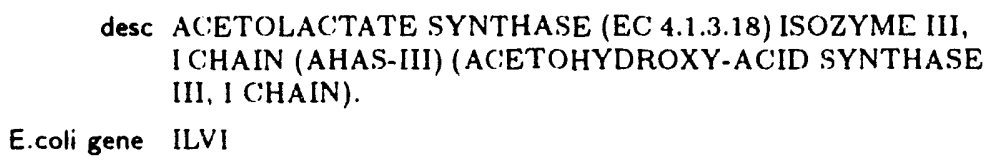

\section{DESCRIPTIONS OF THE CIDS}

desc Acetoliydroxy acid synthase III ; valine-sensitive large subunit

P00894 ilvH 1.8387621 to 88112

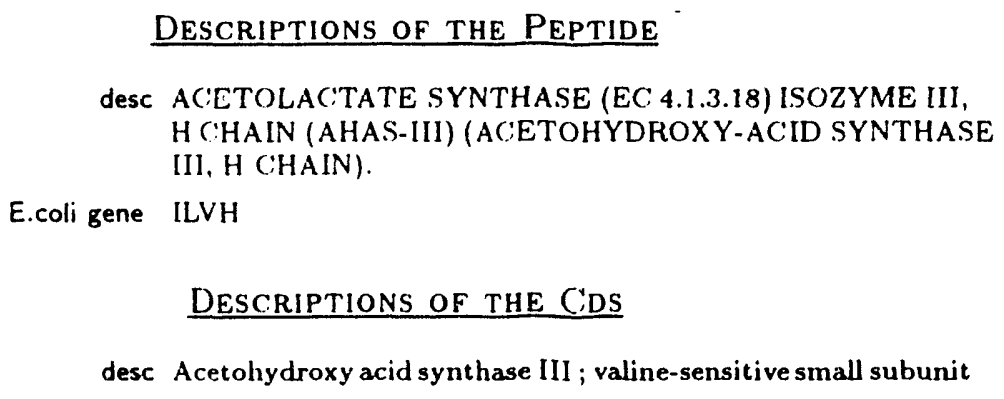

P08142 ilvB 82.693875291 from 3876979 complement

DEscriptions of THE PEPTIDE

desc ACETOLACTATE SYNTHASE (EC: 4.1.3.18) ISOZYME I, LARGE SUBUNIT (AHAS-I) (ACETOHYDROXY-ACID SYNTHASE ().

E.coli gene ILVB

DESCRIPTIONS OF THE CDS

desc Acetohydroxy acid synthase I valine-sensitive large subunit

P08143 ilvN 82.693874997 from 3875287 complement

Descriptions of THE Peptide

desc ACETOLACTATE SYNTHASE (EC 4.1.3.18) ISOZYME I, SMALL SUBUNIT (AHAS-I) (ACETOHYDROXY-ACID SYNTHASE 1).

E.coli gene ILVN 


\section{DESCRIPTIONS OF THE CDS}

desc Acetohydroxy acid synthase $X$; valine-sensitive small subunit

Pl:3048 ilvM 84.90 3980115 to 39800375

Descriptions of the PEPTII)

desc AC'ETOLACTATE SYNTHASE (EC 4.1.3.18) ISOZYME II, SMALL SUBUNIT (AHAS-II) (ACETOHYDROXY-ACID SYNTHASE II).

E.coli gene ILVM

DESCRIPTIONS OF THE CIDS

desc Acetohydroxy acid synthase II : valine-insensitive small subunit

Reaction: 2-aceto-2-Hydroxy-Butyrate_ILE_1 $\begin{aligned} & \text { 2-Aceto-2-Hydroxy-Butyrate } \stackrel{1.1 .1 .86}{=} \begin{array}{l}1-\text { Keto-2-Methylvalerate } \\ \mathrm{NADPH}_{2}\end{array} \\ & \text { NADP }\end{aligned}$

\subsubsection{6: Ketol-Acid Reductoisomerase}

P06168 P05793 P05989

DESCRIPTIONS OF THE ENZYME

desc KETOL-ACID REDUCTOISOMERASE

Alternate name DIHYDROXYISOVALERATE DEHYDROGENASE (ISOMERIZIN(i)

Alternate name ACETOHYDROXY ACID ISOMEROREDUCTASE

Activity 2,3-DIHYDROXYISOVALERATE + NADP $(+)=2$-ACETOLACTATE + NADPH.

P05793 ilvC: 84.913985878 to 3987353

Descriptions of THE PEPTIDE

desC KETOL-ACID REDUCTOISOMERASE (EC 1.1.1.86) (ACETOHYDROXY. ACID ISOMEROREDUC'TASE).

E.coli gene ILVC: 


\title{
DESCRIPTIONS OF THE CDS
}

desc ketol-acid reductoisomerase

Reaction: 1-keto-2-MethylValerate_ILE_1

1-Keto-2-Methylvalerate $\stackrel{4.2 .1 .9}{=} \underset{\mathrm{H}}{=} \mathrm{O}$

\subsubsection{9: Dihydroxy-Acid Dehydratase}

P05791

DESCRIPTIONS OF THE ENZYME

desc. DIHYDROXY-ACID DEHYDRATASE

Activity 2,3-DIHYDROXY-3-METHYLBUTANOATE $=$ 2-OXO-3-METHYLBUTANOATE $+\mathrm{H}(2) \mathrm{O}$.

P05791 ilvD 84.913981389 to 3983236

\author{
DESCRIPTIONS OF THE PEPTIDE \\ desc DIHYDROXY-ACID DEHYDRATASE (EC 4.2.1.9) \\ E.coli gene ILVD \\ DESCRIPTIONS OF THE CDS \\ desc dihydroxyacid dehydratase
}

Reaction: 2-keto-3-Methyl-Valerate_ILE_1

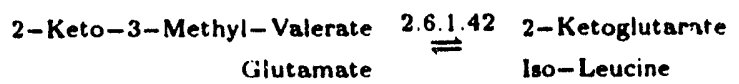

2.6.1.42: Branched-Chain Amino Acid Aminotransferase

P00510 P15168

DESCRIPTIONS OF THE ENZYME

desc BRANCHED-CHAIN AMINO ACID AMINOTRANSFERASE 
Alternate name TRANSAMINASE B

Activity L-LEUCINE + 2-OXOGLUTARATE = 4-METHYL-2-OXOPENTANOATE + L-GLITAMATE.

P00510 ilvE 84.903980395 to 3981324

\section{DESCRIPTIONS OF THE PEPTIDE}

desc BRANC:HED-CHAIN AMINO ACID AMINOTRANSFERASE (EC: 2.6.1.42) (TRANSAMINASE B).

E.coli gene ILVE

\section{DESCRIPTIONS OF THE CDS}

desc branched-chain anino-acid aminotransferase 


\section{Summary of the Pathway: Lysine-Synthesis_1}

\begin{tabular}{|c|c|c|}
\hline $\begin{array}{r}\text { Cilutamate } \\
\text { ८-Aspartate-Semualdehyde } \\
\mathrm{NADPH}_{2} \\
\text { Pyruvate } \\
\text { Succinyl-CoA }\end{array}$ & $\begin{array}{c}1.3 .1 .26 \\
2.3 .1 .117 \\
2.6 .1 .17 \\
3.3 .1 .18 \\
4.1 .1 .20 \\
4.2 .1 .52 \\
\text { s.1.1.7 } \\
\text { Pyridoxal Phosphate }\end{array}$ & $\begin{array}{l}\text { 2-Ketoglutarate } \\
\left(\mathrm{O}_{2}\right. \\
\text { (OA } \\
\text { Lysine } \\
\text { NADP } \\
\text { Succinate }\end{array}$ \\
\hline
\end{tabular}

\section{Reaction: L-Aspartate-Semialdehyde 1}

$$
\begin{aligned}
& \text { L-Aspartate-Semıaldehyde 4.2.1.52 Dihydrodipicolinate } \\
& \text { Pyruvate } \mathrm{H}^{+}
\end{aligned}
$$

\subsubsection{2: Dihydrodipicolinate Synthase}

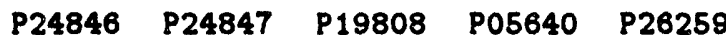

\section{DESCRIPTIONS OF THE ENZYME}

desc DIHYDRODIPICOLINATE SYNTHASE

Alternate name DIHYDRODIPICOLINATE SYNTHETASE

Aiternate name DHDPS

Activity L-ASPARTATE 4-SEMIALDEHYDE + PYRUVATE = DIHYDRODIPICOLINATE $+2 \mathrm{H}(2) \mathrm{O}$.

P05640 dapA 53.242607308 from 2608186 complement

\section{Descriptions of the Peptide}

desc DIHYDRODIPICOLINATE SYNTHASE (EC 4.2.1.52) (DHDPS)

E.coli gene DAPA

\section{DESCRIPTIONS OF THE CDS}

desc Dihydrodipicolinate synthase 
Reaction: Dihydrodipicolinate 1

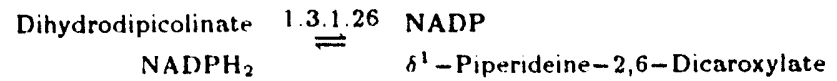

1.3.1.26: Dihydrodipicolinale Reductase

P04036

DESCRIPTIONS OF THE ENZYME

desc DIHYDRODIPICOLINATE REDUCTASE

Activity 2,3,4,5-TETRAHYDRODIPICOLINATE $+\operatorname{NAD}(P)(+)=2,3$ DIHYDRODIPICOLINATE + NAD(P)H.

P04036 dapB 0.5928701 to 29522

DEscriptions OF THE PEPTIDE

desc DIHYDRODIPICOLINATE REDUCTASE (EC 1.3.1.26)

E.coli gene DAPB

DESCRIPTIONS OF THE CDS

desc Dihydrodipicolinate reductase

Reaction: Tetrahydrodipicolinate_1

$$
\begin{array}{rll}
\text { Succinyl-CoA } & \\
\mathrm{H}_{2} \mathrm{O} & \stackrel{2.3 .1 .117}{=} \mathrm{CoA} \\
\delta^{2} \text {-Piperideine-2,6-Dicaroxylate } & & N-\text { Succinyl- } t-\text { Keto- } \alpha-\text { Aminopimelate }
\end{array}
$$

2.3.1.117: 2,3,4,5-Tetrahydropyridine-2-Carboxylate N-Succinyltransferase

DESCRIPTIONS OF THE ENZYME

desc 2,3,4,5-TETRAHYDROPYRIDINE-2-CARBOXYLATE N-SUCCIINYLTRANSFERASE

Alternate name TETRAHYDROPICOLINATE SUCCINYLASE

Activity SUCCINYL-COA + 2,3,4,5-TETRAHYDROPYRIDINE-2-CARBOXYLATE $=$ = $O O A+N-S U C C I I N Y L-L-2-A M I N O-6-O X O H E P T A N E D I O A T E$. 
Pep??? cds??? Pos??? Loc???

Reaction: N-succ-keto-1-Aminopimelate, 1

$\begin{array}{rll}\text { Glutamate } & \text { Pyridoxal Phosphate } & 2 \text { 2-Ketoglutarate } \\ N \text {-Succinyl- } \epsilon-\text { Keto- } \alpha \text {-Aminopimelate } & N \text {-Succinyl-LL-2,6-Diaminopimelate }\end{array}$

2.6.1.17: Succinyldiaminopimelate Aminotransferase

P03948

DESCRIPTIONS OF THE ENZYME

desc SUCY:INYLDIAMINOPIMELATE AMINOTRANSFERASE

Alternate name SUCCINYLDIAMINOPIMELATE TRANSFERASE

Activity N-SUCCINYL-L-2,6-DIAMINOHEPTANEDIOATE + 2-OXOGLUTARATE

= N-SUCCINYL- L-2-AMINO-6-OXOHEPTANEDIOATE + L-CILUTAMATE.

P03948 dapD 3.83190991 from 191815 complement

DESCRIPTIONS OF THE PEPTIDE

desc SUCCINYLDIAMINOPIMELATE AMINOTRANSFERASE

(EC: 2.6.1.17) (SUCCINYLDIAMINOPIMELATE TRANSFERASE).

E.coli gene DAPD

DESCRIPTIONS OF THE CDS

desc Tetralıydrodipicolinate $\mathrm{N}$-succinyltransferase

Reaction: n-succinylll-2,6-diaminopimelate_1

$\begin{aligned} & \mathrm{H}_{2} \mathrm{O} \stackrel{3.5 .1 .18}{\rightleftharpoons} \mathrm{LL-Diaminopimelate} \\ & \text { Succinate }\end{aligned}$

3.5.1.18: Succinyldiaminopimelate Desuccinylase

DESCRIPTIONS OF THE ENZYME

desc SUCCINYLDIAMINOPIMELATE DESUCCINYLASE 


$$
\begin{aligned}
\text { Activity } & \text { N-SUCCINYL-LL-2,6-DIAMINOHEPTANEDIOATE + H(2)O } \\
& =\text { SUCCINATE + LL-2,6-DIAMINOHEP'TANEDIOATE. }
\end{aligned}
$$

Pep??? cds??? Pos??? Loc???

Reaction: LL-Diaminopimelate 1

$$
\text { Lん-Diamincpımelate } \stackrel{5.1 .1 .7}{\rightleftharpoons} \text { Meso-Diaminopımelate }
$$

5.1.1.7: Diaminopimelate Epimerase

P08885

DESCRIPTIONS OF THE ENZYME

- desc DIAMINOPIMELATE EPIMERASE

Activity LL-2,6-DIAMINOHEPTANEDIOATE = MESO-DIAMINOHEPTANEDIOATE.

P08885 dapF 85.714022990 to 4023817

Descriptions OF THE PEPTIDE

desc DIAMINOPIMELATE EPIMERASE (EC, 5.1.1.7)

E.coli gene DAPF

\section{DESCRIPTIONS OF THE CDS}

desc Diaminopimelate epimerase

Reaction: meso-Diaminopimelate 1

$$
\begin{array}{r}
\text { Meso-Diaminopimelate } \stackrel{4.1 .1 .20}{\rightleftharpoons} \mathrm{CO}_{2} \\
\mathrm{H}^{+}
\end{array}
$$

4.1.1.20: Diaminopimelate Decarboxylase

$$
\begin{array}{llll}
\text { P23630 } & \text { P09890 } & \text { P00861 } & \text { P19572 }
\end{array}
$$

DESCRIPTIONS OF THE ENZYME

desC DIAMINOPIMELATE DEC:ARBOXYLASE

Alternate name DAP' DECARBOXYLASE 
Activity MESO-2,6-DIAMINOHEPTANEDIOATE = L-LYSINE + COO(2).

P00861 lysA 61.312995717 from 2996979 complement

DESCRIPTIONS OF THE PEPTIDE

desc DIAMINOPIMELATE DECARBOXYLASE (EC; 4.1.1.20) (DAP DEC:ARBOXYLASE)

E.coli gene LYSA

\section{DESCRIPTIONS OF THE C'DS}

desc Diaminopinelate decarboxylase 


\section{Summary of the Pathway: Methionine-Synthesis_1}

\begin{tabular}{|rcl|}
\hline & 2.1 .1 .13 & Ammonia \\
Cysteine & 4.2 .99 .9 & (.oA \\
Homoserıne & 4.4 .1 .8 & t-Methonine \\
Succinyl-C $: \circ \mathrm{A}$ & & Pyruvate \\
$\mathrm{H}_{2} \mathrm{O}$ & & Succinate \\
& & THF
\end{tabular}

Reaction: Homo-SER_1

$$
\begin{aligned}
& \text { Homoserine 2.3.1.46 CoA } \\
& \text { Succinyl-C:OA } \rightleftharpoons \text { O-Succinyl-L-Homoserine }
\end{aligned}
$$

2.3.1.46: Homoserine Succinyltransferase

P07623

DESCRIPTIONS OF THE ENZYME

desc HOMOSERINE SUCCINYLTRANSFERASE

Alternate name HOMOSERINE O-TRANSSUCCINYLASE

Activity SUCCINYL-COA + L-HOMOSERINE $=r(I M+$ O-SUCCINYLL-HOMOSERINE.

P07623 metA 90.854244462 to 4245391

DESCRIPTIONS OF THE PEPTIDE

desc HOMOSERINE SUCCINYLTRANSFERASE (EC; 2.3.1.46) (HOMOSERINE TRANSSUCCINYLASE).

E.coli gene META

\section{DESCRIPTIONS OF THE CDS}

desc homoserine transsuccinylase

Reaction: O-Succinyl-L-Homoserine_1

\begin{tabular}{rll|}
\hline Cysteine & 4.2 .99 .9 & $L-$ Cystathionine \\
O-Succinyl-L-Homoserine & & Succinate \\
\hline
\end{tabular}

4.2.99.9: O-Succinylhomoserine (Thiol)-Lyase

P00935 


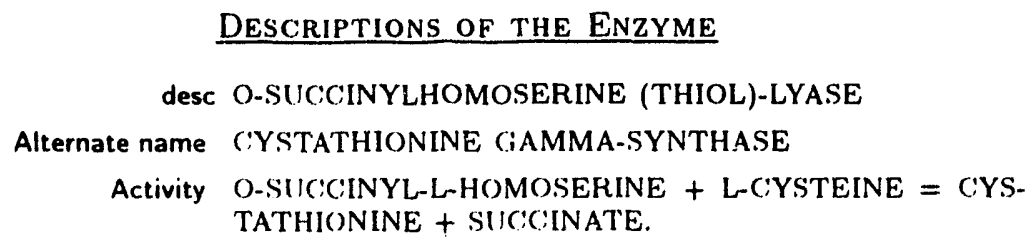

P00935 metB 88.994157834 to 4158994

\section{DEscriptions of THE PEPTIDE}

desc CYSTATHIONINE GAMMA-SYNTHASE (EC 4.2.99.9) (OSUCCINYLHOMOSERINE (THIOL)-LYASE).

E.coli gene METB

DESCRIPTIONS OF THE CDS

desc cystathionine gamma-synthase

Reaction: L-Cystathionine 1

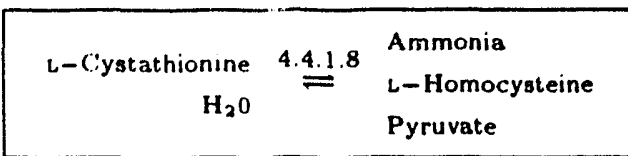

4.4.1.8: Cystathionine-3-Lyase

P06721 P18949

DESCRIPTIONS OF THE ENZYME

desc CYSTATHIONINE-BETA-LYASE

Alternate name BETA-CYSTATHIONASE

Alternate name CYSTEINE LYASE

Activity CYSTATHIONINE $+\mathrm{H}(2) \mathrm{O}=$ L-HOMOCYSTEINE $+\mathrm{NH}(3)$ + PYRUVATE.

P06721 metC; 64.993171650 to 3172837

\section{DESCRIPTIONS OF THE PEPTIDE}

desc CYSTATHIONINE-BETA-LYASE (EC 4.4.1.8) (BETA-CYSTATHIONASE) (CYYSTEINE LYASE). 
E.coli gene METC:

\section{DESCRIPTIONS OF THE CDS}

desc Cystathionine gamma-lyase

\section{Reaction: Homo-CYS_1}

$$
\begin{aligned}
& \text { ( } \mathrm{H}_{3}-\mathrm{THF} \quad 2.1 .1 .13 \text { L-Methionine } \\
& \text { L-Homocysteine } \rightleftharpoons \text { THF }
\end{aligned}
$$

2.1.1.13: 5-Methyltetrahydrofolate-Homocysteine Methyltransferase P25665 P13009

DESCRIPTIONS OF THE ENZYME

desc 5-METHYLTETRAHYDROFOLATE-HOMOCYSTEINE METHYLTRANSFERASE

Alternate name METHIONINE SYNTHASE

Alternate name TETRAHYDROPTEROYLGLUTAMATE METHYLTRANSFERASE

Activity 5-METHYLTETRAHYDROFOLATE + L-HOMOCYSTEINE = TETRAHYDROFOLATE + L-METHIONINE.

P13009 metH 91.054253921 to 4257523

\section{Descriptions of the Peptide}

desc 5-METHYLTETRAHYDROFOLATE-HOMOCYSTEINE METHYLTRANSFERASE, VITAMIN-B12 DEPENDENT ISOZYME (EC 2.1.1.13) (METHIONINE SYNTHASE).

E.coli gene METH

\section{DESCRIPTIONS OF THE CDS}

desc Bl2-dependent homocysteine-N5-methyltetrahydrofolate transmethylase 


\section{Summary of the Pathway: Methionine-Synthesis_2}

\begin{tabular}{|rcl|}
\hline & 2.1 .1 .14 & Ammonia \\
Cysteine & 4.2 .999 & CoA \\
Homoserine & 4.4 .1 .8 & L-Methionine \\
Succinyl-( $\mathrm{H}_{3} \mathrm{~A}$ & & Pyruvare \\
$\mathrm{H}_{2} \mathrm{O}$ & & Succinate \\
& & THF \\
\hline
\end{tabular}

Reaction: Howo-SER_1

$$
\begin{aligned}
& \text { Homoserine 2.3.1.46 C:OA } \\
& \text { Succinyl-CoA } \rightleftharpoons \text { O-Succinyl-L-Homos rine }
\end{aligned}
$$

2.3.1.46: Homoserine Succinyltransferase.

P07623

\section{DESCRIPTIONS OF THE ENZYME}

desc HOMOSERINE SUCCINYITRANSFERASE

Alternate name HOMOSERINE O-TRANSSUCCINYLASE

Activity SUCCINYL-COA + L-HOMOSERINE $=$ COA + O-SUCCINYL L-HOMOSERINE.

P07623 metA 90.854244462 to 4245391

DESCRIPTIONS OF THE PEPTIDE

desc HOMOSERINE SUCCINYLTRANSFERASE (EC 2.3.1.46) (HOMOSERINE TRANSSUCCINYLASE).

E.coli gene META

\section{DESCRIPTIONS OF THE CDS}

desc homoserine transsuccinylase

Reaction: O-Succinyl-L-Homoserine 1

$\begin{array}{rll}\text { Cysteine } & 4.2 .99 .9 & \iota-C y s t a t h i o n i n e \\ \text { O-Succinyl-L-Homoserıne } & & \text { Succinate }\end{array}$

4.2.99.9: O-Succinylhomoserine (Thiol)-Lyase P00935 
DESCRIPTIONS OF THE ENZYME

desC O-SUCCINYLHOMOSERINE (THIOL)-LYASE

Alternate name CYSTATHIONINE GAMMA-SYNTHASE

Activity O-SUCCINYL-L-HOMOSERINE + L-CYSTEINE = CYSTATHIONINE + SUICCINATE.

P00935 metB 88.9941578 .34 to 4158994

Descriptions OF THE PEPTIDE

desC CYSTATHIONINE GAMMA-SYNTHASE (EC 4.2.99.9) (OSUCCINYLHOMOSERINE (THIOL)-LYASE).

E.coli gene METB

DESCRIPTIONS OF THE CDS

desc cystathionine gamma-synthase

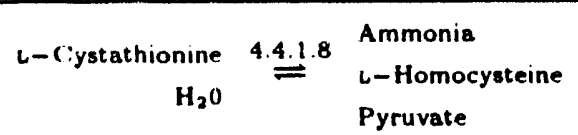

4.4.1.8: Cystathionine-13-Lyase

P06721 P18949

DESCRIPTIONS OF THE ENZYME

desc CYSTATHIONINE-BETA-LYASE

Alternate name BETA-CYSTATHIONASE,

Alternate name CYSTEINE LYASE

Activity CYSTATHIONINE $+\mathrm{H}(2) \mathrm{O}=$ L-HOMOCYSTEINE $+\mathrm{NH}(3)$ + PYRUVATE.

P06721 metc: 64.993171650 to 3172837

\section{DESCRIPTIONS OF THE PEPTIDE}

desc CYSTATHIONINE-BETA-LYASE (EC 4.4.1.8) (BETA-CYSTATHIONASE) (CYSTEINE LYASE). 


\title{
E.coli gene METC: \\ DESCRIPTIONS OF THE CDS
}

desc C.ystathionine ganuma-lyase

\section{Reaction: Homo-CYS_2}

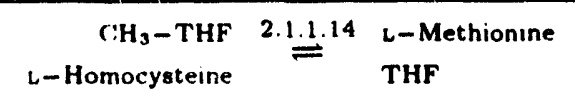

2.1.1.14: 5-Methyltetrahydropteroyltriglutamate-Homocysteine Methyltransferase

\author{
DESCRIPTIONS OF THE ENZYME \\ desc 5-METHYLTETRAHYDROPTEROYLTRIGLUTAMATE-HOMOCYYSTEINE \\ METHYLTRANSFERASE \\ Alternate name TETRAHYDROPTERUYLTRIGLUTAMATE METHYLTRANS- \\ FERASE \\ Activity 5-METHYLTETRAHYDROPTEROYLTRI-L-GLUTAMATE \\ + L-HOMOCYSTEINE = TETRAHYDROPTEROYLTRI- \\ L-CILUTAMATE + LMETHIONINE.
}

Pep??? metE 86.284044010 to 4044074

\section{DESCRIPTIONS OF THE CDS}

desc tetralhydropteroyltriglutanate methyltransferase 


\section{Summary of the Pathway: NAD-Synthesis_1}

$$
\begin{aligned}
& 2.00 * \text { ATP } 2.4 .2 .12 \text { ADP } \\
& \text { Niacinamide } 2.7 .7 .1 \text { NAD } \\
& \begin{array}{ll}
\text { an-5-Phosphsioribosylpp } & P_{i} \\
& 2.00 m P_{i}
\end{array}
\end{aligned}
$$

Reaction: Niacinamide_NAD_1

$$
\begin{array}{rll|}
\hline \text { ATP } & \multicolumn{1}{c|}{\text { ADP }} \\
\text { Niacinamide } & 2.4 .2 .12 & \text { Nicotinamide Nucleotide } \\
\alpha-D-5-P h o s p h o r i b o s y l p p & & P_{i} \\
& & P_{i}
\end{array}
$$

2.4.2.12: Nicotinamide Phosphoribosyltransferase

DESCRIPTIONS OF THE ENZYME

desc NICOTINAMIDE PHOSPHORIBOSYLTRANSFERASE

Alternate name NMN PYROPHOSPHORYLASE

Activity NICOTINAMIDE D-RIBONUCLEOTIDE + PYROPHOS-

PHATE = NICOTINAMIDE + 5-PHOSPHO-ALPHA-D-RIBOSE 1-DIPHOSPHATE.

Pep??? cds??? Pos??? Loc???

Reaction: Nicotinamide_Nucleotide_NAD_1

$$
\text { Nicotinamide Nucleotide } \rightleftharpoons \text { PPi }_{\mathbf{i}}
$$

2.7.7.1: Nicotinamide-Nucleotide Adenylyltransferase

DESCRIPTIONS OF THE ENZYME

desc NICOTINAMIDE-NUCLEOTIDE ADENYLYLTRANSFERASE

Alternate name $N A D(t)$ PYROPHOSPHORYLASE

Activity ATP + NICOTINAMIDE RIBONUCLEOTIDE = PYROPHOSPHATE $+N A D(+)$.

Pep??? cds??? Pos??? Loc??? 


\section{Summary of the Pathway: NAD-Synthesis_2}

\begin{tabular}{|rll|}
\hline $2.00 *$ ATP & 2.4 .2 .19 & AMP \\
Ciulamine & 6.3 .18 & $\mathrm{CO}_{2}$ \\
$\alpha-0-5-$ Phosphoribosylpp & & Cilutamate \\
Quınolinate & & NAD \\
& & $3.00 * \mathrm{PP}_{1}$ \\
\hline
\end{tabular}

\section{Reaction: Quinolinate_NAD_1}

$$
\begin{array}{rll}
\alpha-D-5-P h o s p h o r i b o s y l p p & 2.4 .2 .19 & \begin{array}{l}
\mathrm{CO}_{2} \\
\text { Quinolinate }
\end{array} \\
& \begin{array}{l}
\text { Nicotınate Nucleotide } \\
\mathrm{PP}_{\mathrm{i}}
\end{array}
\end{array}
$$

2.4.2.19: Nicotinate-Nucleotide Pyrophosphorylase (Carboxylating)

\section{DESCRIPTIONS OF THE ENZYME}

desC NICOTINATE-NUCLECTIDE PYROPHOSPHORYLASE (CARBOXYLATING)

Alternate name QUINOLINATE PHOSPHORIBOSYLTRANSFERASE (DECARBOXYLATING)

Activity NIC:OTINATE D-RIBONUCLEOTIDE + PYROPHOSPHATE + $\mathrm{CO}(2)$ = PYRIDINE-2,3-DICARBOXYLATE + 5-PHOSPHOALPHA-D-RIBOSE 1-DIPHOSPHATE.

Pep??? cds??? Pos??? Loc???

Reaction: Nicotinate_Nucleotide_NA.D_1

$\begin{aligned} & \text { ATP } 2.7 .7 .18 \\ & \text { Nicotinate Nucleotide } \text { Deamido-NAD } \\ & \text { PPi }_{i}\end{aligned}$

2.7.7.18: Nicolinale-Nucleotide Adenylyltransferase

DESCRIPTIONS OF THE ENZYME

desc NICOTINATE-NUCLEOTIDE ADENYLYLTRANSFERASE

Aiternate name DEAMIDO-NAD( + ) PYROPHOSPHORYLASE

Activity ATP + NICOTINATE RIBONUCLEOTIDE = PYROPHOSPHATE + DEAMIDO-NAD $(+)$. 
Pep??? cds??? Pos??? Loc???

\section{Reaction: Deamido-NAD_NAD_1}

\begin{tabular}{rll|}
\hline ATP & AMP \\
Deamido-NAD & 6.3.5.1 & Cilutamate \\
Cilutamine & & $\begin{array}{l}\text { NAD } \\
\mathrm{PP}_{\mathbf{i}}\end{array}$ \\
\hline
\end{tabular}

6.3.5.1: NAD Synthase (Glutamine-Hydrolysing)

\section{DESCRIPTIONS OF THE ENZYME}

desc NAD(+) SYNTHASE (GLUTAMINE-HYDROLYSING)

Alternate name $N A D(+)$ SYNTHETASE (GLUTAMINE-HYDROLYSING)

Activity ATP + DEAMIDO-NAD $(+)+$ L-GLUTAMINE + H(2)O $=$ AMP + PYROPHOSPHATE + NAD (+) + L-GLUTAMATE.

Pep??? cds??? Pos???? Loc???? 


\section{Summary of the Pathway: Palmitoyl-ACP-Synthesis_1}

\begin{tabular}{|c|c|c|}
\hline $\begin{array}{r}\text { Acetyl-C:OA } \\
7.00 * \text { Malonyl- }(\because \circ A \\
14.00 * \mathrm{NADPH}_{2}\end{array}$ & $\begin{array}{l}1.1 .1 .100 \\
1.3 .1 .10 \\
2.3 .1 .38 \\
2.3 .1 .39 \\
2.3 .1 .41 \\
4.2 .1 .38 \\
6.4 .1 .2 \\
\text { Biotin } \\
=\end{array}$ & $\begin{array}{l}7.00 * \mathrm{CO}_{2} \\
8.00 * \mathrm{C} .0 A \\
14.00 * \mathrm{NADP} \\
\text { Palmitoyl-ACP } \\
7.00 * \mathrm{H}_{2} \mathrm{O}\end{array}$ \\
\hline
\end{tabular}

Reaction: Acetyl-CoA_Fat_1

\begin{tabular}{|c|c|c|}
\hline Acetyl-CoA & $\stackrel{2.3 .1 .38}{\rightleftharpoons}$ & Acetyl-ACP \\
\hline Acylcarrier-Protein & & CoA \\
\hline
\end{tabular}

2.3.1.38: [Acyl-Carrier Protein] Acetyllransferase

\section{DESCRIPTIONS OF THE ENZYME}

desC [ACYL-CARRIER PROTEIN] ACETYLTRANSFERASE

Activity ACETYL-COA + [ACYLCARRIER PROTEIN] $=$ COA + ACETYL-[ACYL-CARRIER PROTEIN]

Pep??? cds??? Pos??? Loc???

Reaction: Acetyl-CoA_Fat_2

\begin{tabular}{|c|c|c|}
\hline Acetyl-CoA & $\begin{array}{l}\text { 6.4.1.2. } \\
\text { Biotin }\end{array}$ & AI \\
\hline $\begin{array}{r}\text { ATP } \\
\mathrm{HCO}_{3}\end{array}$ & & $\begin{array}{l}\text { Malonyl-CoA } \\
P_{i}\end{array}$ \\
\hline
\end{tabular}

6.4.1.2: Acetyl-CoA Carboxylase.

$\mathrm{P} 24182 \quad \mathrm{P} 02905 \quad \mathrm{P} 11029 \quad \mathrm{P} 11497$

DESCRIPTIONS OF THE ENZYME

desc ACETYLCOA CARBOXYLASE

Activity ATP + ACETYL-COA + HCO(3)(-) = ADP + ORTHOPHOSPHATE + MALONYLCOOA. 
P02905 fabE 70.993425281 to 3425751

\author{
DEscriptions of The PePTIDE \\ desC BIOTIN CAARBOXYL CAARRIER PROTEIN OF ACETYL- \\ COA CARBOXYLASE (EC 6.4.1.2). \\ E.coli gene $\mathrm{ACC} B$ \\ DESCRIPTIONS OF THE CDS
}

desc Acetyl-CoA carboxylase

Reaction: Malonyl-CoA_Fat_1

$$
\begin{aligned}
\text { Acylcarrier-Protein } & 2.3 .1 .39 \\
\text { Malonyl-CoA } & \text { CoA } \\
& \text { Malonyl-ACP }
\end{aligned}
$$

2.3.1.39: [Acyl-Carrier Protein] Malonyltransferase

DESCRIPTIONS OF THE ENZYME

desc [ACYL-CARRIER PROTEIN] MALONYLTRANSFERASE

Activity MALONYL-COA + [AC'YL-CARRIER PROTEIN] = COA + MALONYL-[ACYYL-CARRIER PROTEIN].

Pep??? fabD 24.53 Loc???

DESCRIPTIONS OF THE CDS

desc Mitrayl-C',A-[acyl carrier protein] transacylase

Reaction: Malonyl-ACP_Fat_1

$$
\begin{aligned}
& \text { Acetyl-AC:P } 2.3 .1 .41 \begin{array}{l}
\text { Acetoacetyl-S-ACP } \\
\text { Malonyl-AC:P }
\end{array} \\
& \begin{array}{l}
\text { Acylcarrier-Protein } \\
\mathrm{CO}_{2}
\end{array}
\end{aligned}
$$

2.3.1.41: 3-Oxoacyl-[Acyl-Carrier Protein] Synthase

$\begin{array}{lllll}\mathrm{P} 14926 & \mathrm{P} 23902 & \mathrm{P} 24249 & \mathrm{P} 15368 & \mathrm{P} 19097 \\ \mathrm{P} 12276 & \mathrm{P} 19096 & \mathrm{P} 12785 & & \end{array}$




\section{DESCRIPTIONS OF THE ENZYME}

desc 3-OXOACYL[ACYL-CARRIER PROTEIN] SYNTHASE

Activity ACYL-[AC:YL-CARRIER PROTEIN] + MALONYL-[ACYLCARRIER PROTEIN] = 3-OXOACYL-[AC'YL-CARRIER PROTEIN] + $C O(2)+[$ ACYL-CARRIER PROTEIN].

P14926 fabB Pos???? 2458456 from 2459676 complement

DEscriptions of THE PEPTIDE

desc 3-OXOAC:YL-[AC:YL-CARRIER-PROTEIN] SYNTHASE I (EC 2.3.1.41) (BETA- KETOACYL-ACP SYNTHASE I) (KAS I).

E.coli gene FABB

\section{Reaction: AcetoAcetyl-S-ACP_Fat_1}

$$
\begin{array}{rl}
\text { Acetoacetyl-S-ACP } 1.1 .1 .100 & 3-\mathrm{Hydroxybutyryl-ACP} \\
\mathrm{NADPH}_{2} & \text { NADP }
\end{array}
$$

1.1.1.100: 3-Oxoacyl-[Acyl-Carrier Protein] Reductase

\section{DESCRIPTIUNS OF THE ENZYME}

desC 3-OXOACYLL[ACYYL-CARRIER PROTEIN] REDUCTASE

Activity (3R)-3-HYDROXYACYL-[ACYL-CARRIER PROTEIN] + NADP(+) = 3-OXOACYL- [ACYL-CARRIER PROTEIN] + NADPH.

Pep??? cds??? Pos??? Loc???

\section{Reaction: 3OH-Butyryl-ACP_Fat_1}

$$
\text { 3-Hydroxybutyryl-AC:P }
$$

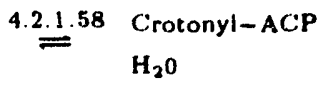

4.2.1.58: Crotonoyl-[Acyl-Carrier Protein] Hydratase

\section{DESCRIPTIONS OF THE ENZYME}

desC CROTONOYL-[ACYLCARRIER PROTEIN] HYDRATASE

Activity (3R)-3-HYDROXYBUTANOYL-[ACYLCARRIER PROTEIN] = BUT-2-ENOYL-[ACYL CARRIER PROTEIN] + H(2)O. 
Pep??? cds??? Pos??? Loc???

Reaction: Crotonyl-ACP_Fat_1

$\begin{aligned} \text { Crotonyl-ACP } & \stackrel{1.3 .1}{=} 10 \text { Butyryl-S-ACP } \\ \mathrm{NADPH}_{2} & \stackrel{\mathrm{NADP}}{=}\end{aligned}$

1.3.1.10: Enoyl-[Acyl-Carrier Protein] Reductase (NADPH, B-Specific)

DESCRIPTIONS OF THE ENZYME

desc ENOYL-[ACYYL-CARRIER PROTEIN] REI)UCTASE (NADPH,

B-SPECIFIC)

Alternate name AC:YL-ACP DEHYDROGENASE

Activity AC:YL-[AC:YL-CARR[ER PROTEIN] + NADP(+) =2,3-DEHYDROACYYL[ACYL-C:ARRIER PROTEIN] + NADPH.

Pep??? cds??? Pos??? Loc??? 


\section{Summary of the Pathway: Pentose-Shunt_1}

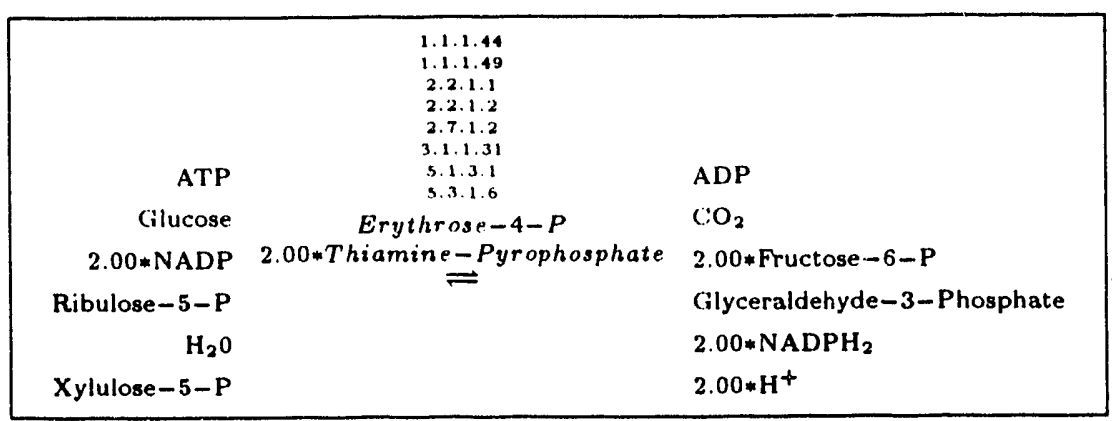

Reaction: glucose_1

$$
\begin{aligned}
\text { ATP } \stackrel{2.7 .1 .2}{=} \begin{array}{l}
\text { ADP } \\
\text { Gilucose }
\end{array} & \begin{array}{l}
\text { ilucose-6-Phosphate } \\
\mathrm{H}^{+}
\end{array}
\end{aligned}
$$

2.7.1.2: Glucokinase

\section{DESCRIPTIONS OF THE ENZYME}

desc GLUCOKINASE

Activity ATP + D-C.ILUCOSE = ADP + D-GLUCOSE 6-PHOSPHATE.

Pep??? glk 51.78 Loc???

DESCRIPTICNS OF THE CDS

desc Cilucokinase

Reaction: glucose-6-P 1

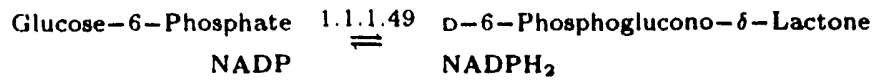

1.1.1.49: Glucose-6-Phosphate Dehydrogenase

$\begin{array}{lllll}\text { P11410 } & \text { P15588 } & \text { P12646 } & \text { P22992 } & \text { P11413 } \\ \text { P11411 } & \text { P05370 } & \text { P11412 } & \text { P21907 } & \end{array}$




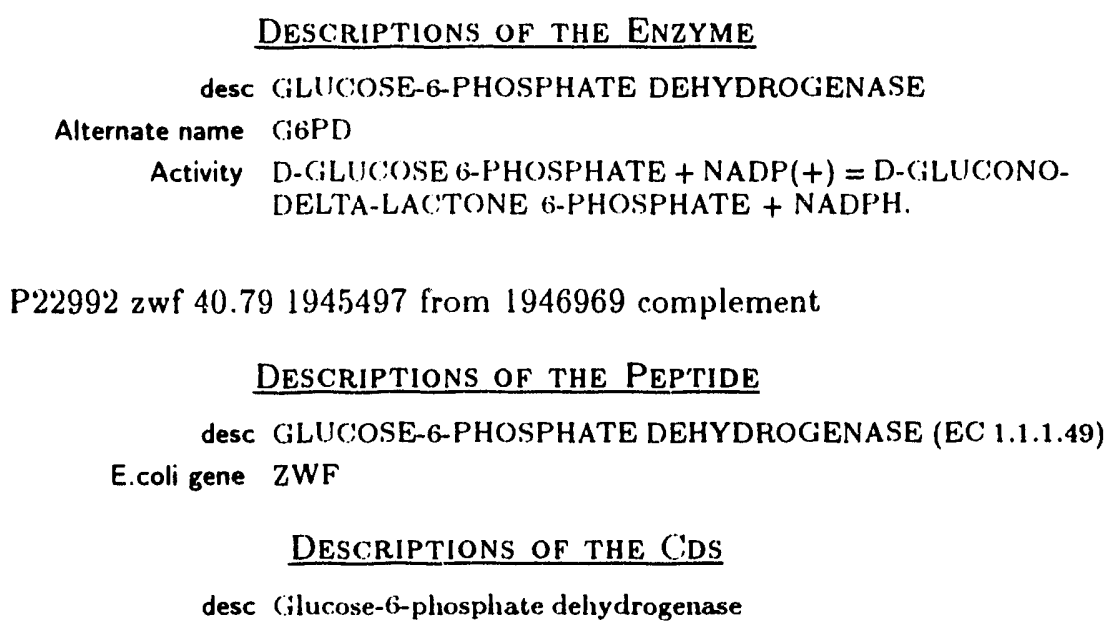

\section{DESCRIPTIONS OF THE C'DS}

desc (ilucose-6-phosphate dehydrogenase

Reaction: 6-P-gluconolactone 1

$$
\begin{array}{rl}
\text { D-6-Phosphoglucono- } \delta \text {-Lactone } & 3.1 .1 .31 \\
\mathrm{H}_{2} \mathrm{O} & 6-\mathrm{P} \text {-Gluconate }
\end{array}
$$

3.1.1.31: 6-Phosphogluconolactonase

\section{DESCRIPTIONS OF THE ENZYME}

desc 6-PHOSPHOCILUCONOLACTONASE

Activity 6-PHOSPHO-D-GLUCONO-1,5-LACTONE + $\mathrm{H}(2) \mathrm{O}=6$ PHOSPHO-D-CILUCONATE.

Pep??? pgl 17.18 Loc???

\section{DESCRIPTIONS OF THE CDS}

desc 6 '-phosphogluconolactonase

Reaction: 6-P-gluconate $\perp$

$$
\begin{aligned}
6-\mathrm{P}-\text { Giluconate } & 1.1 .1 .44 \\
\text { NADP } & \begin{array}{l}
\mathrm{CO}_{2} \\
\mathrm{NADPH}_{2} \\
\text { Ribulose-5-P }
\end{array}
\end{aligned}
$$

1.1.1.44: 6-Phosphogluconate Dehydrogenase (Decarboxylating)

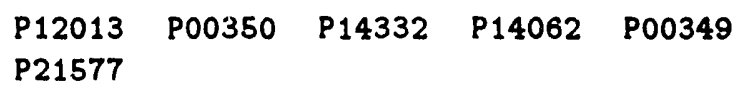




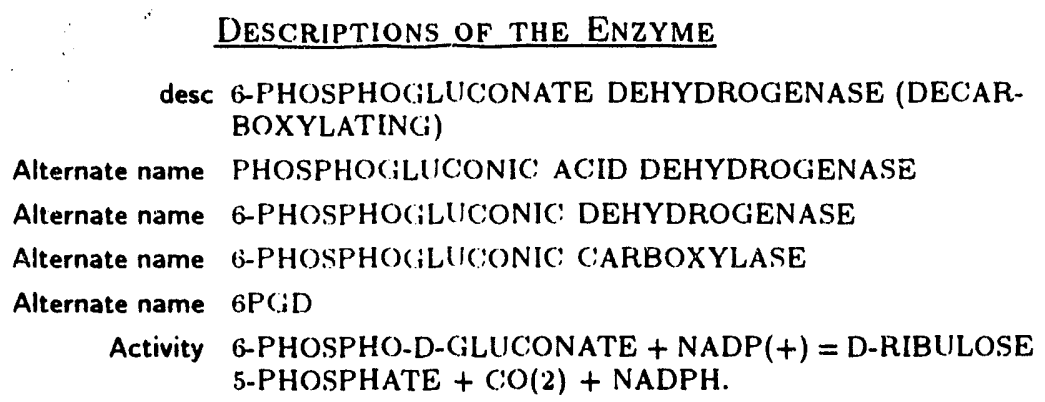

P00350 gnd 44.392111109 from 2112515 complement

Descriptions of THE PEPTIDE

desc 6-PHOSPHOCILUCONATE DEHYDROGENASE, DECARBOXYLATINC: (EC; 1.1.1.44)

E.coli gene (IND

DESCRIPTIONS OF THE CDS

desc Gluconate-6-phosphate dehydrogenase; decarboxylating

Reaction: ribulose-5-P 1

Ribulose-5-P $\stackrel{5.1 .3 .1}{\rightleftharpoons}$ Xylulose-5-P

5.1.3.1: Ribulose-Phosphate 3-Epimerase.

DESCRIPTIONS OF THE ENZYME

desc RIBULOSE-PHOSPHATE 3-EPIMERASE

Alternate name PHOSPHORIBULOSE EPIMERASE

Alternate name ERYTHROSE-4-PHOSPHATE EPIMERASE

Activity D-RIBULOSE 5-PHOSPHATE = D-XYLULOSE 5-PHOSPHATE.

Pep??? cds??? Pos??? Loc???

Reaction: ribulose-5-P_2

$$
\text { Ribulose-5-P } \stackrel{5.3 .1 .6}{\rightleftharpoons} \text { Ribose-5-P }
$$

5.3.1.6: Ribose 5-Phosphate Epimerase 


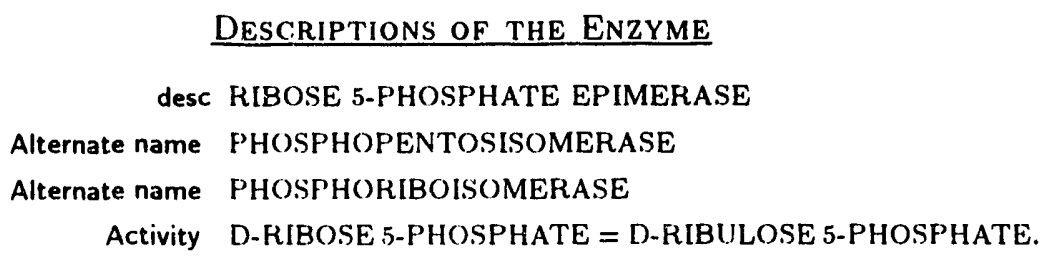

\section{DESCRIPTIONS OF THE CDS}

desc Ribose plosphate isomerase constitutive

Reaction: $x y l u l o s e-5-P \_1$

\begin{tabular}{|c|c|c|}
\hline $\begin{array}{r}\text { Erythrose-4-P } \\
\text { Xylulose-5-P }\end{array}$ & Thiamine-Pyrophosphate & $\begin{array}{l}\text { Fructose-6-P } \\
\text { Gilyceraldehyde-3-Phosphate }\end{array}$ \\
\hline
\end{tabular}

\subsubsection{1: Transketolase}

\section{DESCRIPTIONS OF THE ENZYME}

desc TRANSKETOLASE

Alternate name GLYCOOALDEHYDE TRANSFERASE

Activity SEDOHEPTULOSE 7-PHOSPHATE + D-GLYCERALDEHYDE 3-PHOSPHATE $=$ D-RIBOSE 5-PHOSPHATE + D-XYLULOSE 5-PHOSPHATE.

Pep??? tkt 62.10 Loc???

\section{DESCRIPTIONS OF THE CDS}

desc Transiketolase

Reaction: xylulose-5-P 2

$$
\text { 2.2.1.1 }
$$

Ribose-5-P Thiamine-Pyrophosphate D-Sedoheptulose-7-P

Xylulose-5-P Glyceraldehyde-3-Phosphate

\subsubsection{1: Transketolase}




\section{DESCRIPTIONS OF THE ENZYME}

desc TRANSKETOLASE

Alternate name (iLYCOALDEHYDE TRANSFERASE

Activity SEDOHEPTULOSE 7-PHOSPHATE + D-CILYCERALDEHYDE 3-PHOSPHATE = D-RIBOSE 5-PHOSPHATE + D-XYLULOSE 5-PHOSPHATE.

Pep??? tkt 62.10 Loc???

DESCRIPTIONS OF THE CDS

desc Transketolase

Reaction: sedoheptulose-7-P 1

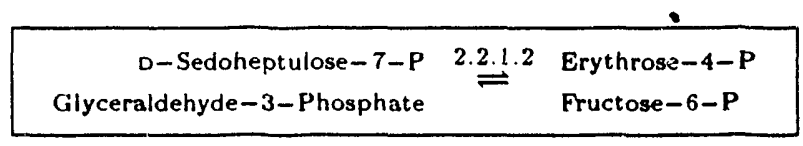

2.2.1.2: Transaldolase

DESCRIPTIONS OF THE ENZYME

desc TRANSALDOLASE

Alternate name DIHYDROXYACETONE TRANSFERASE

Alternaie name GLYCERONE TRANSFERASE

Activity SEDOHEPTULOSE 7-PHOSPHATE + D-GLYCERALDEHYDE 3-PHOSPHATE = D-ERYTHROSE 4-PHOSPHATE + DFRUCTOSE 6-PHOSPHATE.

Pep??? cds??? Pos??? Loc??? 
Summary of the Pathway: Phenylalanine-Synthesis_1

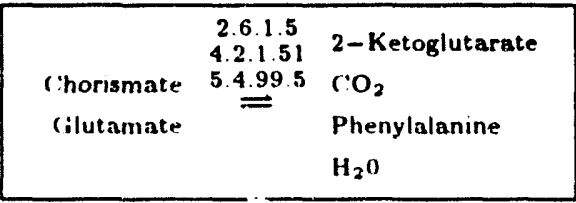

Reaction: Chorismate $\_$

$$
\text { Chorismate } \stackrel{5.4 .15}{\rightleftharpoons} \text { Prephenate }
$$

5.4.99.5: Chorismale Mulase

P19080 P07022 P21204 P07023

DESCRIPTIONS OF THE ENZYME

desc CHORISMATE MIITASE

Activity CHORISMATE = PREPHENATE.

P07022 pheA 56.642750863 to 2752023

Descriptions of The Peptide

desc CHORISMATE MUTASE (EC 5.4.99.5) / PREPHENATE DEHYDRATASE (EC: 4.2.1.51).

E.coli gene PHEA

DESCRIPTIONS OF THE CDS

desc Chorismate mutase-P-prephenate dehydratase

P07023 tyrA 56.642752066 from 2753187 complement

DEscriptions OF THE PEPTIDE

desc CHORISMATE. MUTASE (EC 5.4.99.5) / PREPHENATE DEHYDROGENASE (EC 1.3.1.12).

E.cNi gene TYKA

DESCRIPTIONS OF THE CDS 
Reaction: Prephenate 1

$$
\text { Prephenate } \stackrel{4.2 .1 .51}{=} \begin{aligned}
& \mathrm{CO}_{2} \\
& \text { Phenyl-Pyruvate } \\
& \mathrm{H}_{2} \mathrm{O}
\end{aligned}
$$

4.2.1.51: Prephenate Dehydratase

P21203 P10341 P07022

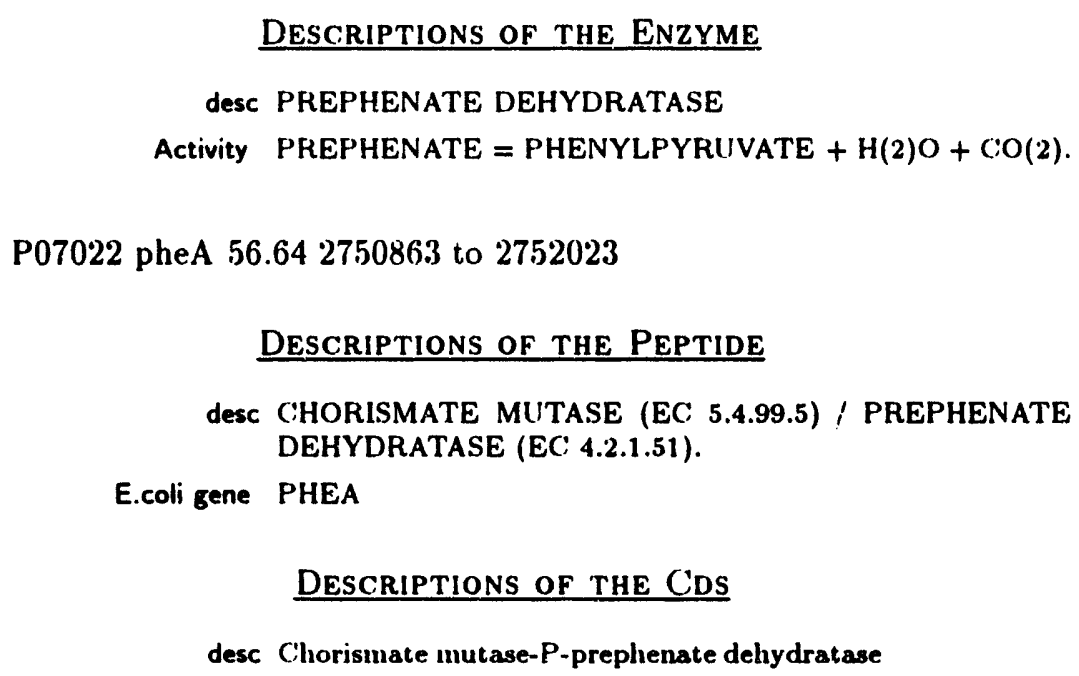

DESCRIPTIONS OF THE ENZYME

desc PREPHENATE DEHYDRATASE

Activity PREPHENATE $=$ PHENYLPYRUVATE $+\mathrm{H}(2) \mathrm{O}+\mathrm{CO}(2)$.

P07022 pheA 56.642750863 to 2752023

DESCRIPTIONS OF THE PEPTIDE

desc CHORISMATE MUTASE (EC 5.4.99.5) i PREPHENATE DEHYDRATASE (EC 4.2.1.51).

E.coli gene PHEA

DESCRIPTIONS OF THE CDS

desc Chorismate mutase-P-prephenate dehydratase

Reaction: Pheuyl-Pyruvate 1

$$
\begin{aligned}
\text { Glutamate } \stackrel{2.6 .1 .5}{=} \begin{array}{l}
2-\text { Ketoglutarate } \\
\text { Phenylalanine }
\end{array} \\
\text { Phenyl-Pyruvate }
\end{aligned}
$$

2.6.1.5: Tyrosine Aminotransferase

DESCRIPTIONS OF THE ENZYME

desc TYROSINE AMINOTRANSFERASE

Alternate name TYROSINE TRANSAMINASE 
Activity L-TYROSINE + 2-OXOC:LUTARATE $=4$-HYDROXYPHENYLPYRUVATE + L-GLUTAMATE.

Pep??? tyrB 91.984296866 to 4298059

\section{DESCRIPTIONS OF THE (DS}

desc Tyrosine aminotransferase; tyrosine repressible 


\section{Summary of the Pathway: Proline-Synthesis_1}

\begin{tabular}{|rcl|}
\hline & 1.2 .1 .41 & ADP \\
ATP & 1.5 .1 .2 & $2.00 *$ NADP \\
Gilutamate & 2.7 .2 .11 & $\mathrm{P}_{\mathrm{i}}$ \\
$2.00 * \mathrm{NADPH}_{2}$ & & Proline \\
& & $\mathrm{H}_{2} \mathrm{O}$ \\
\hline
\end{tabular}

Reaction: GLT_2

\begin{tabular}{|c|c|c|}
\hline ATP & $\stackrel{2.7 .2 .11}{=}$ & \\
\hline Glutamat & & 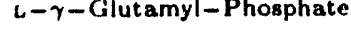 \\
\hline
\end{tabular}

2.7.2.11: Glutamate 5-Kinase.

P07005 P17856

DESCRIPTIONS OF THE ENZYME

desc GLUTAMATE 5-KINASE

Alternate name GAMMA-GLUTAMYL KINASE

Activity ATP + L-GLUTAMATE = ADP + L-GLUTAMATE 5-PHOSPHATE.

P07005 proB 5.90267708 to 268811

DESCRIPTIONS OF THE PEPTIDE

desc GLUTAMATE 5-KINASE (EC 2.7.2.11)(GAMMA-GLUTAMYL KINASE) (GK)

E.coli gene PROB

DESCRIPTIONS OF THE C.DS

desc gamma-glutanyl kinase

Reaction: L-gamma-Glutamyl-Phosphate_1

$$
\begin{aligned}
\llcorner-\gamma-\text { Glutamyl-Phosphate } & 1.2 .1 .41 \\
\mathrm{NADPH}_{2} & \begin{array}{l}
\text { L-Glutamate } \gamma-\text { Semialdehyde } \\
\text { NADP } \\
P_{i}
\end{array}
\end{aligned}
$$

1.2.1.41: Glutamate-5-Semialdehyde Dehydrogenase

P07004 P17857 


\section{DESCRIPTIONS OF THE ENZYME}

desc (SLUTAMATE-5-SEMIALDEHYDE DEHYDROGENASE

Alternate name BETA-(iLUTAMYLPHOSPHATE REDUCTASE

Activity L-GLUTAMATE 5-SEMIALDEHYDE + ORTHOPHOSPHATE + NADP $(+)=$ L-GAMMA- GLUTAMYL PHOSPHATE + NADPH.

P07004 proA 5.90268823 to 270046

Descriptions of the Peptide

desc (iAMMA-GLUTAMYL PHOSPHATE REDUCTASE (GPR) (EC: 1.2 .1 .41 ) (GLUTAMATE-5- SEMIALDEHYDE DEHYDRO(IENASE).

$$
\text { E.coli gene PROA }
$$

\section{DESCRIPTIONS OF THE C.DS}

desc ganuna-glutamyl phosphate reductase

\section{Reaction: L-Glutamate_gamma-Semialdehyde_1}

$$
\text { L-Glutamate } \gamma \text {-Semialdehyde } \rightleftharpoons \begin{aligned}
& \mathrm{H}_{2} \mathrm{O} \\
& \mathrm{L}-1 \text {-Pyrroline-5-Carboxylate }
\end{aligned}
$$

No enzymes have been found to catalyze this reaction.

\section{Reaction: 1-delta(1)-pyrroline_5-carboxylate_1}

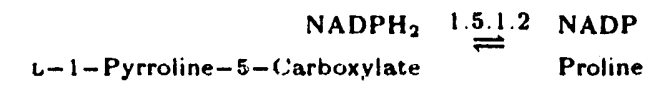

1.5.1.2: Pyrroline-5-Carboxylate Reductase

$$
\begin{array}{lllll}
\mathrm{P} 14383 & \mathrm{P} 00373 & \mathrm{P} 22350 & \mathrm{P} 22008 & \mathrm{P} 17817
\end{array}
$$

DESCRIPTIONS OF THE ENZYME

desc PYRROLINE-5-CARBOXYLATE REDUCTASE

Alternate name $\mathrm{P} 5 \mathrm{CR}$

Activity L-PROLINE + NAD $(P)(+)=1-$ PYRROLINE-5-CARBOXYLATE $+\mathrm{NAD}(\mathrm{P}) \mathrm{H}$. 


\section{P00373 proC' 8.86411644 from 412453 complement \\ Descriptions of the Peptide \\ desc PYRROLINE-5-CARBOXYLATE REDUCTASE (EC 1.5.1.2) (P5CR) \\ E.coli gene $\mathrm{P}^{\prime} \mathrm{ROC}$ \\ DEscriptions OF THE CDS \\ desc pyrroline-5-carboxylate reductase}




\section{Summary of the Pathway: Purine-Synthesis_1}

\begin{tabular}{|c|c|c|}
\hline $\begin{array}{r}2.00 * 10 \text { - Formyl-THF } \\
9.00 * \text { ATP } \\
\left(\mathrm{O}_{2}\right. \\
3.00 * \text { (ilutamine } \\
\text { (ilycine } \\
\text { IMP } \\
2.00 * \text { L-Aspartate } \\
\text { NAD } \\
\text { Ribuse-5-P } \\
3.00 * \mathrm{H}_{2} \mathrm{O}\end{array}$ & $\begin{array}{c}1.1 .1 .203 \\
2.1 .2 .2 \\
2.1 .2 .3 \\
2.4 .2 .14 \\
2.7 .4 .3 \\
2.7 .4 .6 \\
2.7 .4 .6 \\
2.7 .6 .1 \\
3.5 .4 .10 \\
4.1 .1 .21 \\
4.3 .2 .2 \\
6.3 .2 .6 \\
6.3 .3 .1 \\
6.3 .4 .13 \\
6.3 .4 .4 \\
6.3 .5 .2 \\
6.3 .3 .3 \\
A T P \\
2.00 * H_{2} 0 \\
=\end{array}$ & $\begin{array}{l}8.00 * A D P \\
2.00 * A M P \\
2.00 * \text { Fumarate } \\
\text { GDP } \\
3.00 * \text { Gilutamate } \\
\text { NADH } \\
5.00 * P_{i} \\
2.00 * P P_{i} \\
2.00 * \text { THF }\end{array}$ \\
\hline
\end{tabular}

Reaction: IMP-Synthesis 1

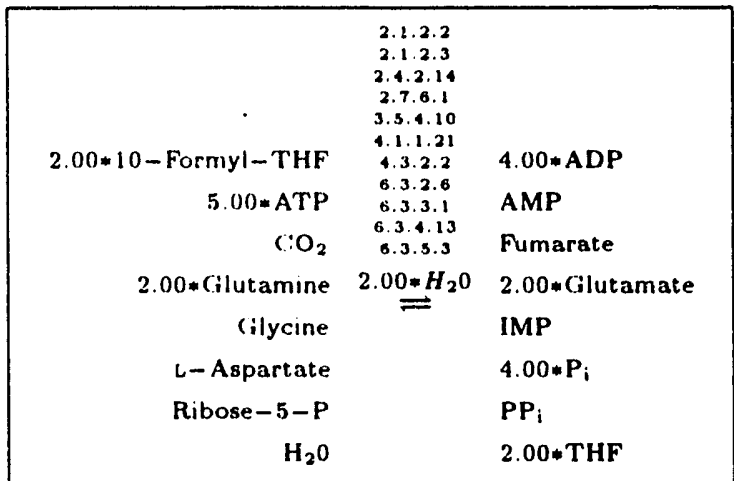

2.1.2.2: Phosphoribosylglycinamide Formyltransferase

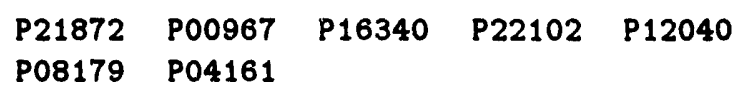

DESCRIPTIONS OF THE ENZYME

desc PHOSPHORIBOSYLGLYCINAMIDE FORMYLTRANSFERASE Alternate name 5 '-PHOSPHORIBOSYLGLYCINAMIDE TRANSFORMYLASE 


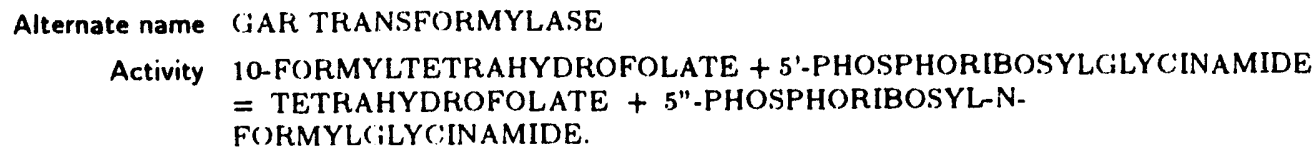

P08179 purN 53.80 2630216 to 26308554

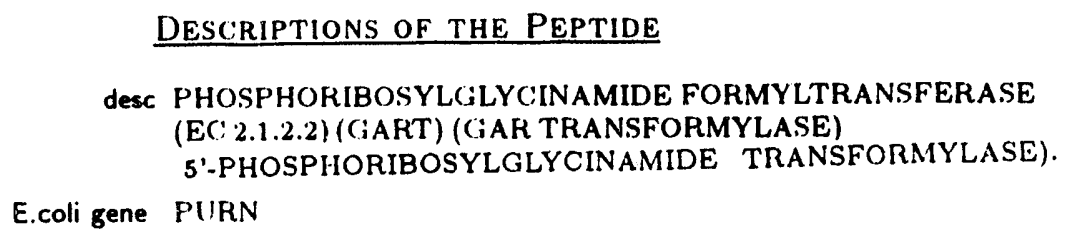

DESCRIPTIONS OF THE CDS

desc 5'-phosphoribosyglycinanide transformylase

2.1.2.3: Phosphoribosylaminoimidazolecarboxamide Formyltransferase

P12048 P15639

\section{DESCRIPTIONS OF THE ENZYME}

desc PHOSPHORIBOSYLAMINOIMIDAZOLECARBOXAMIDE FORMYLTRANSFERASE

Alternate name AICAR TRANSFORMYLASE

Activity 10-FORMYLTETRAHYDROFOLATE + 5'-PHOSPHORIBOSYL5-AMINO-4- IMIDAZOLECARBOXAMIDE = TETRAHYDROFOLATE + 5"-PHOSPHORIBOSYL-5- FORMAMIDO4-IMIDAZOLEC:ARBOXAMIDE.

P15639 purH 90.504236127 from 4237716 complement

Descriptions of The Peptide

desc PHOSPHORIBOSYLAMINOIMIDAZOLECARBOXAMIDE FORMYLTRANSFERASE (EC 2.1.2.3) (AICAR TRANSFORMYLASE) / IMP CYCLOHYDROLASE (EC 3.5.4.10).

E.coli gene PURH

\section{DESCRIPTIONS OF THE CDS}

desc phosphoribosylanninoimidazolecarboxamide formyltransferase 


\subsubsection{4: Amidophosphoribosyltransferase}

P00497 P00496 P04046

\section{DESCRIPTIONS OF THE ENZYME}

desc AMIL)(OFHOSFHORIBOSYLTRANSFERASE

Alternate name (iLIITAMINE PHOSPHORIBOSYLPYROPHOSPHATE AMIDOTRANSFERASE

Alternate name PHOSPHORIBOSYLDIPHOSPHATE 5-AMIDOTRANSFERASE

Activity 5-PHOSPHO-BETA-D-RIBOSYLAMINE + PYROPHOSPHATE + L-GLUTAMATE = L-CiLUTAMINE + 5-PHOSPHO-ALPHAD-RIBOSE 1-DIPHOSPHATE + H(2)O.

P00496 purF 50.002446890 from 2448407 complement

\section{Descriptions of the Peptide}

desC AMIDOPHOSPHORIBOSYLTRANSFERASE (EC 2.4.2.14) (GLUTAMINE PHOSPHORIBOSYLPYROPHOSPHATE AMIDOTRANSFERASE).

E.coli gene PURF

\section{DESCRIPTIONS OF THE CDS}

desc amidophosphoribosyl transferase

2.7.6.1: Ribose-Phosphate Pyrophosphokinase

$\begin{array}{lllll}\mathrm{P} 09329 & \mathrm{P} 11908 & \mathrm{P} 09330 & \mathrm{P} 21108 & \mathrm{P} 14193 \\ \mathrm{P} 08330 & \mathrm{P} 15849 & & & \end{array}$

DESCRIPTIONS OF THE ENZYME

desc RIBOSE-PHOSPHATE PYROPHOSPHOKINASE

Alternate name PHOSPHORIBOSYL PYROPHOSPHATE SYNTHETASE

Activity ATP + D-RIBOSE 5-PHOSPHATE = AMP + 5-PHOSPHOALPHA-D-RIBOSE 1-DIPHOSPHATE.

P08330 prs 26.491268223 from 1269170 complement

Descriptions of the Peptide

desc RIBOSE-PHOSPHATE PYROPHOSPHOKINASE (EC 2.7.6.1) (PHOSPHORIBOSYL PYROPHOSPHATE SYNTHETASE).

E.coli gene PRS 


\section{DESCRIPTIONS OF THE CDS}

desc Phosphoribosylpyrophosphate synthetase

3.5.4.10: IMP C'yclohydrolase

P12048 P15639

DESCRIPTIONS OF THE ENZYME

desc IMP (:YCLOHYDROLASE

Activity IMF $+\mathrm{H}(2) \mathrm{O}=5$-FORMAMIDO-1-(5-PHOSPHORIBOSYL)IMIDAZOLE4-CARBOXAMIDE.

P15639 purH $90.5042: 36127$ from 42:37716 complement

DESCRIPTIONS OF THE PEPTIDE

desc PHOSPHORIBOSYLAMINOIMIDAZOLECARBOXAMIDE FORMYLTRANSFERASE (EC 2.1.2.3) (AICAR TRANSFORMYLASE) / IMP (YYCLOHYDROLASE (EC 3.5.4.10).

E.coli gene PURH

\section{DESCRIPTIONS OF THE CDS}

desc phosphoribosylanninoinudazolecarboxamide formyltransferase

4.1.1.21: Phosphoribosylaminoimidazole Carboxylase

$\begin{array}{lllll}\text { P12044 } & \text { P09028 } & \text { P22234 } & \text { P15567 } & \text { P21264 } \\ \text { P12046 } & \text { P09029 } & & & \end{array}$

DESCRIPTIONS OF THE ENZYME

desc PHOSPHORIBOSYLAMINOIMIDAZOLE CARBOXYLASE

Alternate name AIR CARBOXYLASE

Activity 1-(5-PHOSPHORIBOSYL)-5-AMINO-4-IMIDAZOLECARBOXYLATE $=1-(5-$ PHOSPHORIBOSYL)-5-AMINOIMIDAZOLE + CO(2).

P09028 purE 11.61558926 from 559435 complement

DESCRIPTIONS OF THE PEPTIDE

desc PHOSPHORIBOSYLAMINOIMIDAZOLE CARBOXYLASE C:ATALYTIC SUBUNIT (EC 4.1.1.21) (AIR CARBOXYLASE). 
E.coli gene PURE

DESCRIPTIONS OF THE CDS

desc phosphoribosylaninoinudazole carboxylase catalytic subunit

P09029 purK 12.19557862 from 558929 complement

Descriptions of the Peptide

desc PHOSPHORIBOSYLAMINOIMIDAZOLE CARBOXYLASE CO(2) FIXATION SUBUNIT (EC 4.1.1.21) (AIR CARBOXYLASE).

E.coli gene PURK

\section{DESCRIPTIONS OF THE ('DS}

desc phosphoribosylatninoinidazole carboxylase; $\mathrm{CO}_{2}$-fixing subunit

4.3.2.2: Adenylosuccinate Lyase

DESCRIPTIONS OF THE ENZYME

desc ADENYLOSUCCINATE LYASE

Alternate name ADENYLOSUCCINASE

Activity ADENYLOSUCCINATE = FUMARATE + AMP.

Pep??? purB 25.42 Loc???

$$
\text { L. CRIPTIONS OF THE C.DS }
$$

desc adenylosuccinate lyase

\subsubsection{6: Phosphoribosylaminoimidazole-Succinocarboxamide Synthase}

P22234 P12046 P21155

DESCRIPTIONS OF THE ENZYME

desc PHOSPHORIBOSYLAMINOIMIDAZOLE-SUCCINOCARBOXAMIDE SYNTHASE 


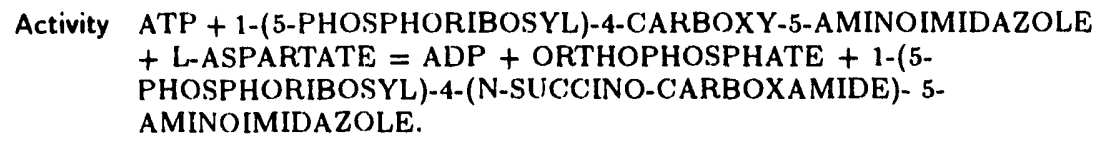

P.21155 pur(: 53.1926053331 from 2606044 complement

\section{DESCRIPTIONS OF THE PEPTIDE}

desc PHOSPHORIBOSYlamiNOIMIDAZOLE-SUCCINOC:ARBOXAMIDE SYNTHASE (EC; 6.3.2.6) (SAICAR SYNTHETASE).

E.coli gene PURC:

\section{DESCRIPTIONS OF THE CDS}

desc phosphoribosylaninoinudazole-succinocarboxamidesynthetase

\subsubsection{1: Phosphoribosylformylglycinamidine Cyclo-Ligase}

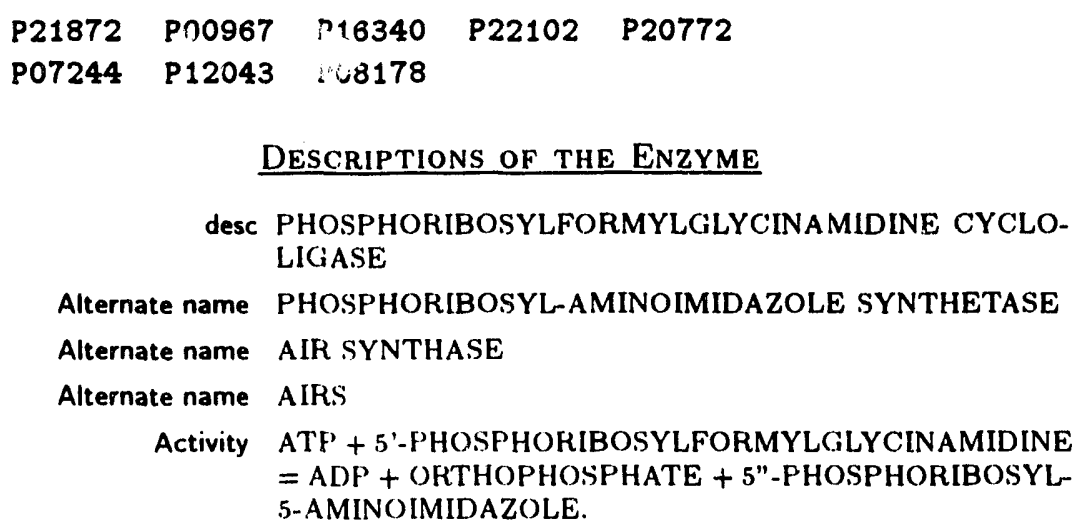

desc PHOSPHORIBOSYLFORMYLGLYCINAMIDINE CYCLOLIGASE

Alternate name PHOSPHORIBOSYL-AMINOIMIDAZOLE SYNTHETASE

Alternate name AIR SYNTHASE

Alternate name AIRS

Activity ATF' + 5'-PHOSPHORIBOSYLFORMYLGLYCINAMIDINE $=$ ADP + ORTHOPHOSPHATE + 5"-PHOSPHORIBOSYL5-AMINOIMIDAZOLE.

P08178 purM 5.3.80 2629179 to 26.30216

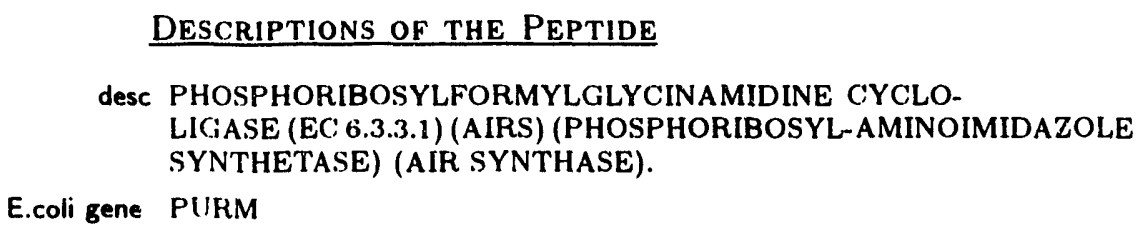

\section{DESCRIPTIONS OF THE C'DS}

desc phosphoribosylaninoinidazole synthetase homologous to purl of S. typhimurium 
6.3.4.13: Phosphoribosylamine-Cilycine Ligase.

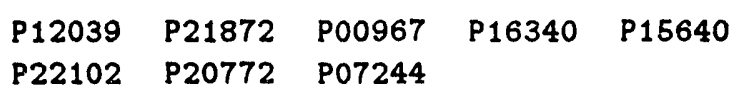

DESCRIPTIONS OF THE ENZYME

desc P'HOSPHORIBOSYLAMINE-GLYCINE LIGASE

Alternate name PHOSPHORIBOSYLGLYCINAMIDE SYNTHETASE

Alternate name CLYCINAMIDE RIBONUCLEOTIDE SYNTHETASE

Alternate name GiARS

Activity ATP + 5-PHOSPHORIBOSYLAMINE + GLYCINE = ADP + ORTHOPHOSPHATE + 5"-PHOSPHORIBOSYLGLYCINAMIDE.

P15640 purD 90.504234826 from 4236115 complement

DESCRIPTIONS OF THE PEPTIDE

desc PHOSPHC : RIBOSYLAMINE-GLYCINE LIGASE (EC 6.3.4.13) (GARS) (GLYCINAMIDE RIBONUCLEOTIDE SYNTHETASE) (PHOSPHORIBOSYLGLYCINAMIDE SYNTHETASE).

E.coli gene PURD

\section{DESCRIPTIONS OF THE CDS}

desc phosphoribosylglycineanide synthetase

\subsubsection{3: Phosphoribosylformylglycinamidine Synthase}

\section{P12041 P12042 P15254}

DESCRIPTIONS OF THE ENZYME

desc PHOSPHORIBOSYLFORMYLGLYCINAMIDINE SYNTHASE

Alternate name PHOSPHORIBOSYLFORMYLGLYCINAMIDINE SYNTHETASE

Alternate name F(iAM SYNTHASE

Activity ATP + 5'-PHOSPHORIBOSYLFORMYLGLYCINAMIDE + L-GLITAMINE + H(2)O = ADP + ORTHOPHOSPHATE $+5 "$-PHOSPHORIBOSYLFORMYLGLYCINAMIDINE + LGLUTAMATE.

P15254 purL 55.20 2701613 frum 2705500 complement

DESCRIPT'JNS OF THE PEPTIDE 
desc PHOSPHORIBOSYLFORMYLCILYCINAMIDINE SYNTHASE (EC: 6.3.5.3) (FGAM SYNTHASE).

E.coli gene PlIRL

\section{DESCRIPTIONS OF THE CDS}

desc phosphoribosylformylglycineanide synthetase ; homologous to purf $i$ of S. typhimurimu

\section{Reaction: ATP-Synthesis_1}

\begin{tabular}{|c|c|c|}
\hline ATP & $\begin{array}{l}2.7 .4 .3 \\
2.7 .6 .6 \\
4.3 .2 .2 \\
6.3 .4 .4\end{array}$ & $2.00 *$ ADP \\
\hline (;TP & ATP & Fumarate \\
\hline IMP & & (iDP \\
\hline L-Aspartate & & $\mathbf{P}_{\mathbf{i}}$ \\
\hline
\end{tabular}

2.7.4.3: Adenylate Kinase

$\begin{array}{lllll}\text { P00570 } & \text { P05081 } & \text { P00568 } & \text { P00571 } & \text { P00569 } \\ \text { P07170 } & \text { P08166 } & \text { P16304 } & \text { P12115 } & \text { P05082 } \\ \text { P24323 } & \text { P10251 } & \text { P } 10772 & & \end{array}$

DESCRIPTIONS OF THE ENZYME

desc ADENYLATE RINASE

Alternate name MY()KINASE

Activity $A T P+A M P=A D P+A D P$.

P05082 adk 10.89 Loc????

DESCRIPTIONS OF THE PEPTIDE

desc ADENYLATE KINASE (EC:2.7.4.3) (ATP-AMP TRANSPHOSPHORYLASE)

E.coli gene ADK

\section{DESCRIPTIONS OF THE CDS}

desc adenylate kinase activity; pleiotropic effects on glycerol-3phosphate acyltranserase activity

2.7.4.6: Nucleoside-Diphosphate Kinase 
DESCRIPTIONS OF THE ENZYME

desc NIICLEOSIDE-DIPHOSPHATE KINASE

Alternate name NUCLEOSIDE 5'-DIP'HOSPHATE PHOSPHOTRANSFERASE

Alternate name NDK

Activity ATP' + NIIC 'LEOSIDE DIP'HOSPHATE $=A$ AP $^{3}+$ NIICLEOSIIIE TRIPHOSPHATE.

Pep??? cds??"? Pos?"?"? Loc?"?"?

4.3.2.2: Adenylosucrinr1' Lyase

DESCRIPTIONS OF THE ENZYME

desc ADENYLOSUCCINATE LYASE

Alternate name ADENYLOSIICC:INASE

Activity ADENYLOSUICCINATE = FUMARATE + AMP.

Pep??? purB 25.42 Loc????

DESCRIPTIONS OF THE CDS

desc adenylosuccinate lyase

\subsubsection{4: Adenylosuccinate Siynthass}

P21900 P12283

DESCRIPTIONS OF THE ENZYME

desc ADENYLOSUCCENATE SYNTHASE

Alternate name IMP-ASPAKTATE LIC IASE

Activity ('TP' + IMI' + L-ASPAKTATE = GDP + ORTHOPHOSPHATE + ADENYLOSIIC:INATE.

Pl2283 purA 95.004435934 to 4437232

Descriptions of the PEPTIDE

desc ADENYLOSUCCINATE SYNTHETASE (EC 6.3.4.4) (IMPASPARTATE LIGASE)

E.coli gene PUIRA

DESCRIPTIONS OF THE CDS 
Reaction: GTP-Synthesis_1

$\begin{array}{rcl}\text { 3.00*ATP } & 11.1 .205 & 2.00 . A D P \\ \text { Gilutamine } & 2.7 .4 .8 & \text { AMP } \\ \text { IMP } & 6.3 .5 .2 & \text { Glutamate } \\ \text { NAD } & & \text { GTP } \\ 2.00 . \mathrm{H}_{2} \mathrm{O} & & \mathrm{NADH}_{2} \\ & & \text { PPi }\end{array}$

1.1.1.205: IMP Dehydrogenase.

$\begin{array}{lllll}\text { P20839 } & \text { P12268 } & \text { P21879 } & \text { P06981 } & \text { P21620 }\end{array}$

P12269 P24547

DESCRIPTIONS OF THE ENZYME

desc IMP DEHYDROGENASE

Alternate narne INOSINE-5'-MONOPHOSPHATE DEHYDROGENASE

Activity INOSINE 5'-PHOSPHATE + NAD(+) + H(2)O = XANTHOSINE 5 -PHOSPHATE + NADH

P0698: guaB 53.90 26405.54 from 2642020 complement

DESC IPTIONS OF THE PEPTIDE

desC INOSINE-5'-MONOPHOSPHATE DEHYDROGENASE (EC 1.1.1.205) (IMP DEHYDROGENASE) (IMPDH).

E.coligene GUAB

DESCRIPTIONS OF THE CDS

desc IMP dehydrogenase

2.7.4.6: Nucleoside-Diphosphate Kinase

DESCRIPTIONS OF THE ENZYME

desc NUCLEOSIDE-DIPHOSPHATE KINASE

Alternate name NIICLEOSIDE 5'-DIPHOSPHATE PHOSPHOTRANSFERASE

Alternate name NDK 
Activity ATP + NUCLEOSIDE DIPHOSPHATE = ADP + NUCLEOSIDE TRIPHOSPHATE.

Pep??? cds???? Pos??? Loc???

2.7.4.8: Guanylate Kinass

\section{DESCRIPTIONS OF THE ENZYME}

desc (jUANYLATE KINASE

Alternate name DEOXY(iUANYLATE KINASE

Activity ATP + GMP = ADP + GDP.

Pep??? cds??? Pos??? Loc???

\subsubsection{2: GMP Synthase (Glulamine-Hydrolysing)}

P04079

DESCRIPTIONS OF THE ENZYME

desc GMP SYNTHASE (GLUTAMINE-HYDROLYSING)

Alternate name GMP SYNTHETASE (GLUTAMINE-HYDROLYSING)

Alternate name GiLUTAMINE AMIDOTRANSFERASE

Activity ATP + XANTHOSINE 5'-PHOSPHATE + LGLUTAMINE

$+\mathrm{H}(2) \mathrm{O}=\mathrm{AMP}+$ PYHOPHOSPHATE + GMP + LGLUTAMATE.

P04079 guaA 53.90 2638908 from 2640485 complement

DESCRIPTIONS OF THE PEPTIDE

desc (IMP SYNTHASE (CILITTAMINE-HYDROLYSING) (EC 6.3.5.2) ((ILIITAMINE AMIDOTRANSFERASE).

E.coli gene (illAA

\section{DESCRIPTIONS OF THE CDS}

desc CiMP synthetase 


\section{Summary of the Pathway: Pyrimidine-Synthesis_1}

\begin{tabular}{|c|c|c|}
\hline $\begin{array}{r}\text { Ainmonium } \\
5.00 * \text { ATP } \\
\text { Gilutamine } \\
\text { L-Aspartate } \\
\mathrm{O}_{2} \\
\alpha-D-5-\text { Phosphoribosylpp }\end{array}$ & $\begin{array}{c}1.3 .3 .1 \\
2.1 .3 .2 \\
2.4 .2 .10 \\
2.7 .4 .6 \\
3.5 .2 .3 \\
4.1 .1 .23 \\
6.3 .4 .2 \\
6.3 .5 .5 \\
\text { Iridyiatekinate } \\
\mathrm{COO}_{2} \\
\stackrel{\mathrm{H}_{2} \mathrm{O}}{=}\end{array}$ & $\begin{array}{l}5.00 * A D P \\
\text { C.TP } \\
\text { Glutamate } \\
3.00 * \mathrm{Pi}_{i} \\
\mathrm{PP}_{\mathrm{i}} \\
\mathrm{H}_{2} \mathrm{O}\end{array}$ \\
\hline
\end{tabular}

Reaction: Glutamine.Pyrimidine 1

\begin{tabular}{|rll|}
\hline $2.00 * \mathrm{ATP}$ & - & $2.00 * \mathrm{ADP}$ \\
$\mathrm{CO}_{2}$ & 6.3 .5 .5 & (arbamoyl-Phosphate \\
Glutamine & & (ilutamate \\
$\mathrm{H}_{2} \mathrm{O}$ & & $\mathrm{P}_{\mathrm{i}}$ \\
\hline
\end{tabular}

\subsubsection{5: Carbamoyl-Phosphate Synthase (Glutamine-Hydrolysing)

$\begin{array}{lllll}\text { P25993 } & \text { P00907 } & \text { P22572 } & \text { P20597 } & \text { P14845 } \\ \text { P07258 } & \text { P25994 } & \text { P00968 } & \text { P13258 } & \text { P14846 } \\ \text { P03965 } & \text { P18185 } & \text { P20054 } & \text { P05990 } & \text { P08955 } \\ \text { P07259 } & & & & \end{array}$

DESCRIPTIONS OF THE ENZYME

desc CARBAMOYL-PHOSPHATE SYNTHASE (GLUTAMINEHYDROLYSING)

Alternate name CARBAMOYL-PHOSPHATE SYNTHETASE (GLUTAMINEHYDROLYSINGi)

Alternate name GLUTAMINE-DEPENDENT CARBAMOYL-PHOSPHATE SYNTHASE

Alternate name GD-CPSASE

Activity 2 ATP + (iLUTAMINE + $\mathrm{CO}(2)+\mathrm{H}(2) \mathrm{O}=2 \mathrm{ADP}+$ ORTHOPHOSPHATE + GLUTAMATE + CARBAMOYL PHOSPHATE.

P00907 carA 0.6829978 to 31126

\section{Descriptions of the Peptide}


desc CARBAMOYL-PHOSPHATE SYNTHASE SMALL CHAIN (EC; 6.3.5.5) (C:ARBAMOYL- PHOSPHATE SYNTHETASE GLUTAMINE ('HAIN).

E.coli gene C:ARA

\section{DESCRIPTIONS OF THE (.DS}

desc carbanoylphosphate synthase;glutamine (light) subunit

P00968 carB 0.6831144 to $34: 365$

DEscriptions OF THE PEPTIDE

desc ('ARBAMOYL-PHOSPHATE SYNTHASE LARGE CHAIN (EC: 6.3.5.5) (C:ARBAMOYL PHOSPHATE SYNTHETASE AMMONIA ('HAIN).

E.coli gene $C$ :ARB

DESCRIPTIONS OF THE C'DS

desc carbamoylphosphate synthase;ammonia (heavy) subunit

Reaction: Carbamoyl-P_Pyrimidine_1

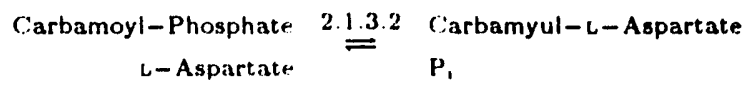

2.1.3.2: Aspartate C'arbamoyltransferase

$\begin{array}{lllll}\text { P20054 } & \text { P05990 } & \text { P08955 } & \text { P07259 } & \text { P05654 } \\ \text { P00479 } & \text { P08420 } & \text { P19910 } & \text { P00478 } & \text { P08421 } \\ \text { P19936 } & & & & \end{array}$

DESCRIPTIONS OF THE ENZYME

desC ASPARTATE C:ARBAMOYLTRANSFERASE

Alternate name ASPARTATE TRANSCARBAMYLASE

Alternate name CARBAMYLASPARTOTRANSKINASE

Alternate name ATC:ASE

Activity CARBAMOYL PHOSPHATE + L-ASPARTATE = ORTHOPHOSPHATE + N-CARBAMOYL-L ASPARTATE.

P00478 pyrl 96.494500898 from 4501359 complement 


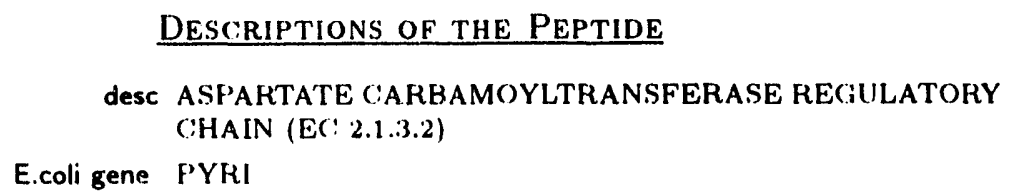

DESCIRIPTIONS OF THE C.DS

desc Aspartate carbamoyltransferase regulatory subunit

P00479 pyrB 96.494501372 from 4502307 complement

DESCRIPTIONS OF THE PEPTIDE

desc ASPARTATE (:ARBAMOYLTRANSFERASE C:ATALYTIC: (:HAIN (E(: 2.1.3.2) (ATC:ASE)

E.coli gene P'YRB

\section{DESCRIPTIONS OF THE CDS}

desc Aspartate carbamoyltransferase catalytic subunit

\section{Reaction: Carbamoyl-L-Aspartate.Pyrimidine_1}

$$
\text { Carbamyul-L-Aspartate } \stackrel{3.5 .2 .3}{=} \begin{aligned}
& \text { Dihydro-Orotate } \\
& \mathrm{H}_{2} \mathrm{O}
\end{aligned}
$$

\subsubsection{3: Dihydroorotase.}

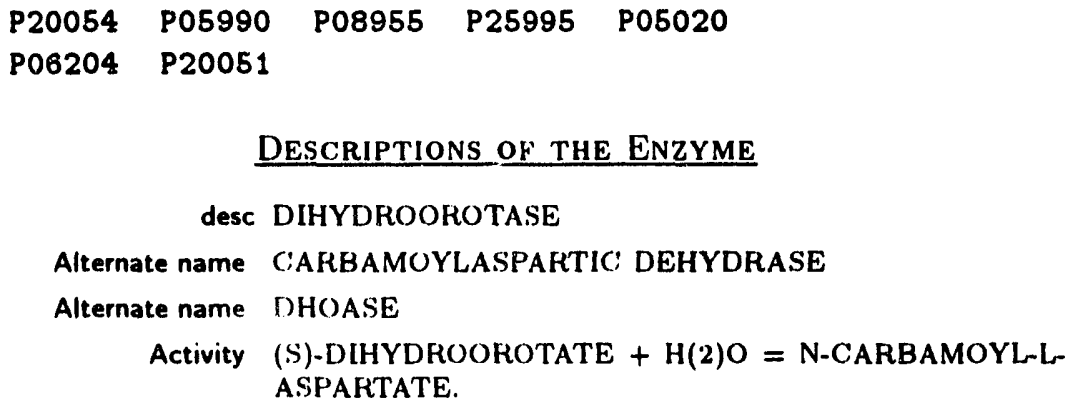

DESCRIPTIONS OF THE ENZYME

desc DIHYDROOROTASE

Alternate name CARBAMOYLASPARTIC: DEHYDRASE

Alternate name DHOASE

Activity (S)-DLHYDROOROTATE + H(2)O = N-CARBAMOYL-L A.SPARTATE.

P05020 pyrC: 23.761130819 from 1131865 complement

Descriptions of the Peptide 


$$
\begin{aligned}
& \text { desc DIHYDROOROTASE (EC: } 3.5 .2 .3 \text { ) } \\
& \text { E.coli gene PYRC: } \\
& \text { DESCRIPTIONS OF THE (DS } \\
& \text { desc Dilydroorotase }
\end{aligned}
$$

Reaction: Di-H-Orotate_Pyrimidine_1

$$
\begin{aligned}
& \text { Dihydro-Orotate } \stackrel{1.3 .3 .1}{=} \text { Orotate } \\
& \mathrm{O}_{2} \mathrm{H}_{2} \mathrm{O}
\end{aligned}
$$

\subsubsection{1: Dihydroorotate Oxidase}

$$
\text { P25996 P05021 P25468 }
$$

\section{DESCRIPTIONS OF THE ENZYME}

desc DIHYDROOROTATE OXIDASE

Activity L-DIHYDROOROTATE $+\mathrm{O}(2)=$ OROTATE $+\mathrm{H}(2) \mathrm{O}(2)$.

P05021 pyrD 21.241012030 to 1013040

\section{Descriptions OF THE Peptide}

desc DIHYDROOROTATE DEHYDROGENASE (EC 1.3.3.1) (DIHYDROOROTATE OXIDASE)

E.coligene PYRD

\section{DESCRIPTIONS OF THE CDS}

desc Diliydroorotate oxidase

\section{Reaction: Orotate_Pyrimidine 1}

$$
\begin{aligned}
& \text { Orotate } \stackrel{2.4 .2 .10}{=} \mathrm{PP}_{\mathrm{i}} \\
& \alpha-\mathrm{D}-5-\mathrm{Phosphoribosylpp} \text { Orotidine-5'-Phosphate }
\end{aligned}
$$

2.4.2.10: Orotale Phosphoribosyltransferase

$\begin{array}{lllll}\text { P09556 } & \text { P11172 } & \text { P13439 } & \text { P25972 } & \text { P18132 } \\ \text { P00495 } & \text { P08309 } & \text { P08870 } & \text { P18904 } & \text { P21846 } \\ \text { P13298 } & & & & \end{array}$




\section{DESCRIPTIONS OF THE ENZYME}

desC OROTATE PHOSPHORIBOSYLTRANSFERASE

Alternate name OROTIDYLIC A(:ID PHOSPHORYLASE

Alternate name (OROTIDINE-5'-P'HOSP'HATE PYROPHOSPHORYLASE

Activity OROTIDINE 5'-PHOSPHATE + PYROPHOSPHATE = ORO-

TATE + 5-F'HOSP'HO-ALP'HA- D-RIBOSE 1-DIPHOSPHATE.

P00495 pyrE 82.043838254 from 3838892 complement

DESCRIPTIONS OF THE PEPTIDE

desC OROTATE PHOSPHORIBOSYLTRANSFERASE (EC 2.4.2.10) (OPRT)

E.coli gene P'YKE

DESCRIPTIONS OF THE (DS

desc Orotate phosphoribosyltransferase

Reaction: orotidine-5'-p_Pyrimidine 1

$$
\text { Orotidine-5'-Phosphate } \stackrel{4.1 .1 .23}{\rightleftharpoons} \begin{aligned}
& \mathrm{CO}_{2} \\
& \text { UMP }
\end{aligned}
$$

4.1.1.23: Orotidine-5'-Phosphate Decarboxylase

$\begin{array}{lllll}\text { P07817 } & \text { P10652 } & \text { P25971 } & \text { P13649 } & \text { P } 14017 \\ \text { P08244 } & \text { P07922 } & \text { P24220 } & \text { P05035 } & \text { P09463 } \\ \text { P21593 } & \text { P07691 } & \text { P } 14964 & \text { P14965 } & \text { P } 21594 \\ \text { P15188 } & \text { P03962 } & \text { P09556 } & \text { P11172 } & \text { P } 13439\end{array}$

DESCRIPTIONS OF THE ENZYME

desc OROTIDINE-5'-PHOSPHATE DECARBOXYLASE

Alternate name OROTIDYLIC: DECARBOXYLASE

Activity OROTIDINE 5'-PHOSPHATE = UMP + CO(2).

P08244 pyrF 28.741350962 to 1351699

DESCRIPTIONS OF THE PEPTIDE 


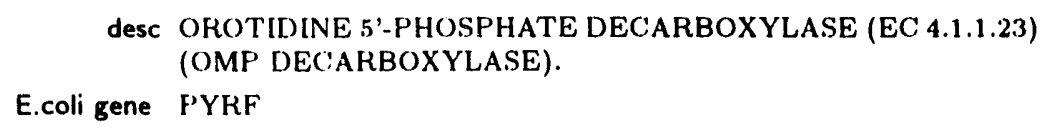

\section{DESCRIPTIONS OF THE (DS}

desc ()rotidline-5'-phosphate decarboxylase

Reaction: UMP_Pyrimidine_1

\begin{tabular}{|ll}
\hline ATP & Uridylatekinase \\
UMP & ADP \\
& UDP
\end{tabular}

Uridylate kinase

Pep???? cds??? Pos??? Lor???

Reaction: UDP Pyrimidine 1

$$
\begin{array}{lll}
\text { ATP } & 2.7 .4 .6 & \text { ADP } \\
\text { UDP } & & \text { UTP }
\end{array}
$$

2.7.4.6: Nucleoside-Diphosphate Kinase.

DESCRIPTIONS OF THE ENZYME

desc NIICLEOSIDE-DIPHOSPHATE KINASE

Alternate name NUC'LEOSIDE 5'-DIPHOSPHATE PHOSPHOTRANSFERASE

Aiternate name $\mathrm{NDK}$

Activity ATP + NUCLEOSIDE DIPHOSPHATE = ADP + NUCLEOSIDE TRIPHOSPHA'TE.

Pep??? cds??? Pos??? Loc???

Reaction: UTP_Pyrimidine_1

\begin{tabular}{|c|c|c|}
\hline Ammonium & 6.3 .4 .2 & ADP \\
\hline ATP & $\rightleftharpoons$ & СТP \\
\hline UTP & & $P_{i}$ \\
\hline
\end{tabular}

6.3.4.2: CTP Synthase

P13242 P08398 P17812 


\section{DESCRIPTIONS OF THE ENZYME}

desc ('TP' SYNTHASE

Alternate name ITP-AMMONIA LICiASE

Activity ATP + $1 \mathrm{ITP}^{\prime}+\mathrm{NH}(3)=\mathrm{ADP}+$ ORTHOPHOSPHATE + ('TP'.

P08398 pyr(; 59.70 Loc????

\section{DEscriptions of the PEPTIDE}

desc CTP SYNTHASE (EC 6.3.4.2) (UTP-AMMONIA LIGASE) (CTP-SYNTHETASE)

E.coli gene PYRG:

DESCRIPTIONS OF THE ('DS

desc ('TH' synthetase 


\section{Summary of the Pathway: Serine_1}

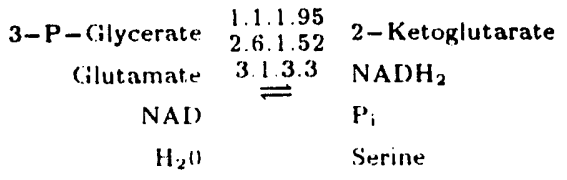

\section{Reaction: G3P_SER_1}

3-P-Cilycerate 1.1.95 3-P-Hydroxypyruvate

$\mathrm{NAL} \rightleftharpoons \mathrm{NADH}_{2}$

1.1.1.95: Phosphoglycerate Dehydrogenase.

P08328

DESCRIPTIONS OF THE ENZYME

desC PHOSPHOCLYCERATE DEHYDROGLNASE

Activity 3-PHOSPHOCLYCERATE $+\mathrm{NAD}(+)=3$-PHOSPHOHYDROXYPYRUVATE $+\mathrm{NADH}$.

P08328 serA 62.993075076 from 3076308 complement

DEscriptions OF THE PEPTIDE

desc D-3-PHOSPHO(ILYCERATE DEHYDROCGENASE (EC 1.1.1.95) (P(iDH)

E.coli gene SERA

DESCRIPTIONS OF THE CDS

desc D-3-phosphoglycerate dehydrogenase

Reaction: 3-P-HydroxyPyruvate_SER_1

3-P-Hydroxypyruvate 2.6.1.52 2-Ketoglutarate

(ilutamate 3 -P-Serine

2.6.1.52: Phosphoserine Aminotransferase

$\begin{array}{llll}\text { P10658 } & \text { P23721 } & \text { P17902 } & \text { P1968 }\end{array}$ 


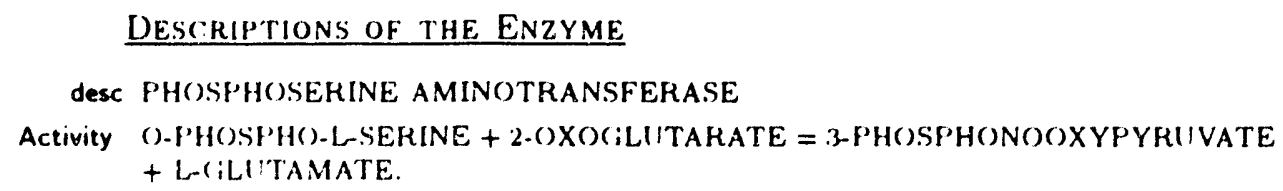

Reaction: 3-P-Serine_SER_1

$$
\begin{aligned}
& \text { 3-P-Serine } \stackrel{3.13 .3}{=} P_{\text {s }} \\
& \mathrm{H}_{2} \mathrm{O} \rightleftharpoons \text { Serine }
\end{aligned}
$$

\subsubsection{3: Phosphoserine Phosphalase}

P06862

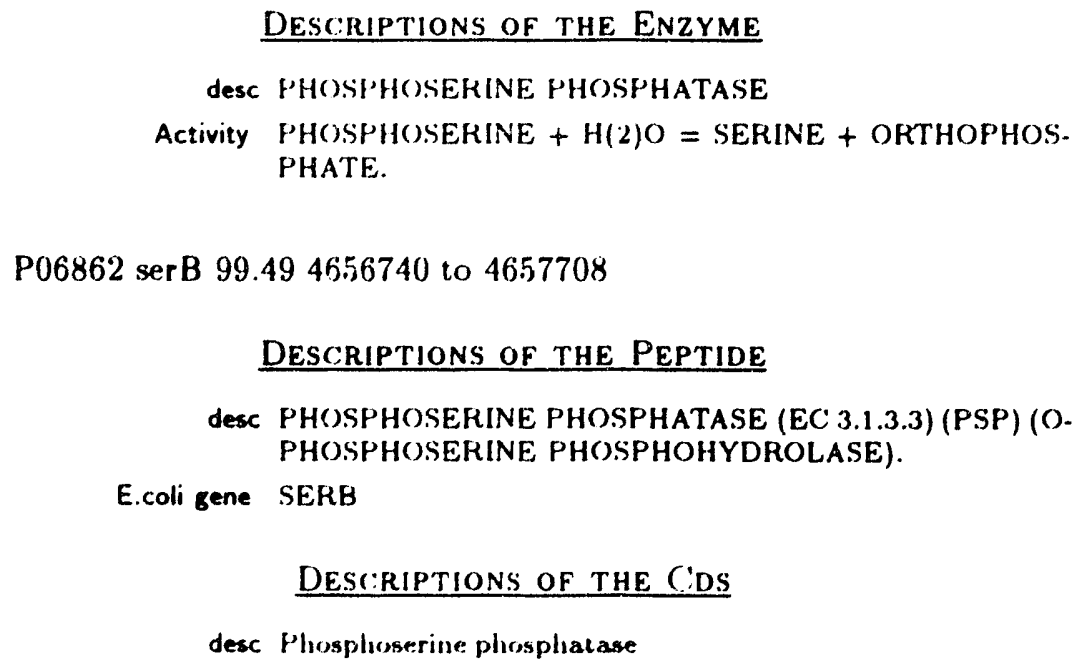




\section{Summiny of the Pathway: TCA_1}

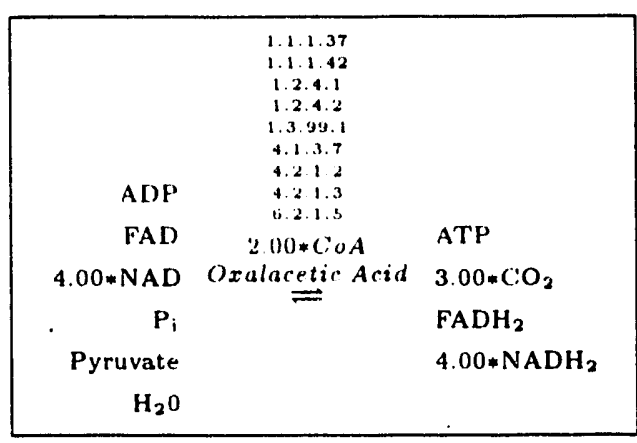

Reaction: pyruvate_2

$$
\begin{array}{rll}
\mathrm{COA} & 1.2 .4 .1 & \text { Acetyl-CoA } \\
\mathrm{NAL} & \rightleftharpoons & \mathrm{CO}_{2} \\
\mathrm{NADH}_{2}
\end{array}
$$

1.2.4.1: Pyruvate Dehydrogenase (Lipoamide)

$$
\begin{array}{lllll}
\text { P26267 } & \text { P10801 } & \text { P06958 } & \text { P26268 } & \text { P21873 } \\
\text { P21881 } & \text { P08559 } & \text { P26284 } & \text { P16387 } & \text { P26269 } \\
\text { P21874 } & \text { P21882 } & \text { P11966 } & \text { P11177 } &
\end{array}
$$

\section{DESCRIPTIONS OF THE ENZYME}

desc PYRUVATE DEHYDROGENASE (LIPOAMIDE)

Alternate name PYRUVATE DECARBOXYLASE

Alternate name PYKUVIC: DEHYDROCENASE

Alternate name PYRUVIC: DEHYDROCIENASE

Activity PYRUVATE + LIPOAMIDE = S-ACETYLDIHYDROLIPOAMIDE $+\operatorname{CO}(2)$.

P06958 aceE 2.78123544 to 126204

Descriptions of the Peptide

desc PYRUVATE DEHYDROGENASE E1 COMPONENT (EC 1.2.4.1)

E.coli gene ACEE 


\section{DESCRIPTIONS OF THE C.DS}

desc pyruvate delydrogenase (decarboxylase component);acetate requirement

Reaction: acetyl CoA_1

$$
\begin{aligned}
& \text { Acetyl-CoA } \stackrel{4.1 .3 .7}{=} \text { Citrate } \\
& \text { Oxalacetic Acid }=\mathrm{CoA}
\end{aligned}
$$

4.1.3.7: Citrate (Si)-Synthase

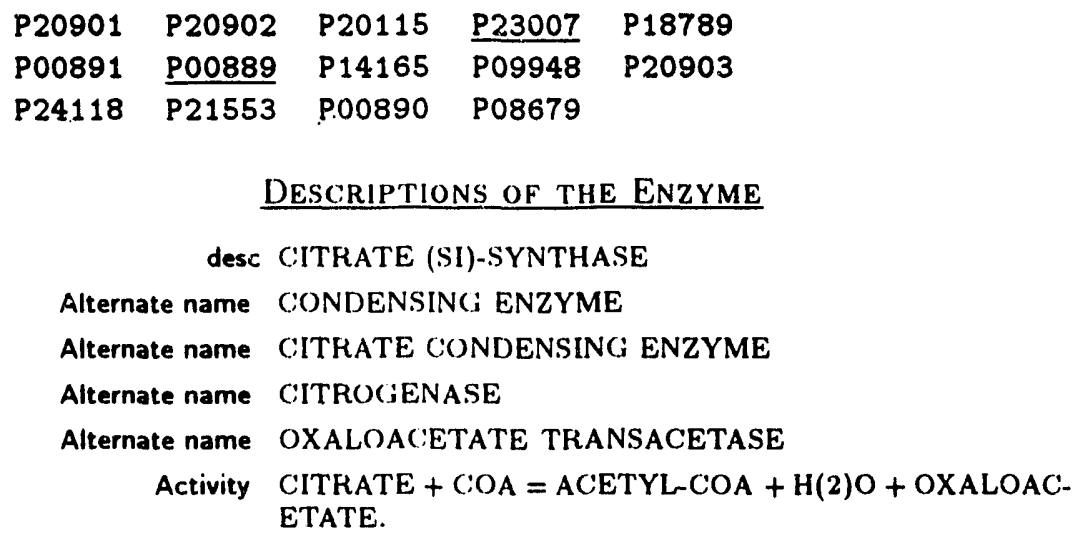

DESCRIPTIONS OF THE ENZYME

desc CITRATE (SI)-SYNTHASE

Alternate name CONDENSING ENZYME

Alternate name CITRATE CONDENSING ENZYME

Alternate name CITROCIENASE

Alternate name OXALOACETATE TRANSACETASE

Activity CITRATE + COA = ACETYLCOA + H(2)O + OXALOACETATE.

P00891 gltA 16.37763390 from 764673 complement

DEsCRIPTIONS OF THE PEPTIDE

desc CITRATE SYNTHASE (EC; 4.1.3.7)

E.coli gene GLTA

\section{DESCRIPTIONS OF THE C.DS}

desc citrate synthase

Reaction: citrate 1

$$
\text { Citrate } \stackrel{4.2 .1 .3}{=} 180-\text { Citrate }
$$

4.2.1.3: Aconitate Hydratase 


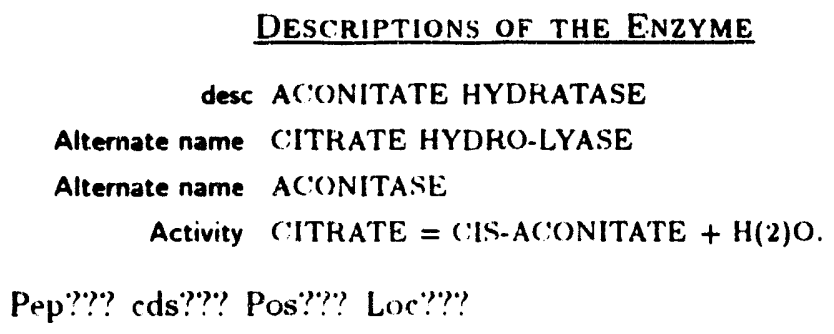

Reaction: isocitrate_1

$$
\begin{aligned}
\text { lso-citrate } & 1.1 .1 .42 \\
\mathrm{NAD} & \begin{array}{l}
2-\text { Ketoglutarate } \\
\mathrm{CO}_{2} \\
\mathrm{NADH}_{2}
\end{array}
\end{aligned}
$$

1.1.1.42: Isocilrale Dehydrogenase (NADP+)

$$
\text { P16100 P08200 P20304 P80046 P21954 }
$$

DESCRIPTIONS OF THE ENZYME

desc ISOCITRATE DEHYDROGENASE (NADP+)

Alternate name OXALOSIIC:IINATE DEC:ARBOXYLASE

Alternate name IDH

Activity D-ISOCITRATE + NADP $(+)=2$-OXOGLUTARATE + CO(2) + NADPH.

P08200 iedC Pos???? 1217957 to 1218121

Descriptions of the Peptide

desc ISOCITKATE DEHYDROGENASE (NADP) (EC 1.1.1.42) (OXALOSIICCINATE DECARBOXYLASE) (IDH).

E.coli gene ICDC:

E.coli gene IC.DE

P08200 icdE Pos??? 1200162 to 1201412

Descriptions of THE Peptide

desc ISOC:ITRATE DEHYDHOC:ENASE (NADP) (EC 1.1.1.42) (OXALOSUIC(INATE DECARBOXYLASE) (IDH).

E.coli gene ICDC: 
E.coli gene IC:DE

Reaction: 2-ketoglutarate 1

$$
\begin{aligned}
& \text { 2-Ketoglutarate } \quad 1.2 .4 \mathrm{CO}_{2} \\
& \text { (OA } \stackrel{1.2 .4 .2}{=} \mathrm{NADH}_{2} \\
& \text { NAD Succinyl-CoA }
\end{aligned}
$$

1.2.4.2: Oxoglutarate Dehydrogenase (Lipoamide)

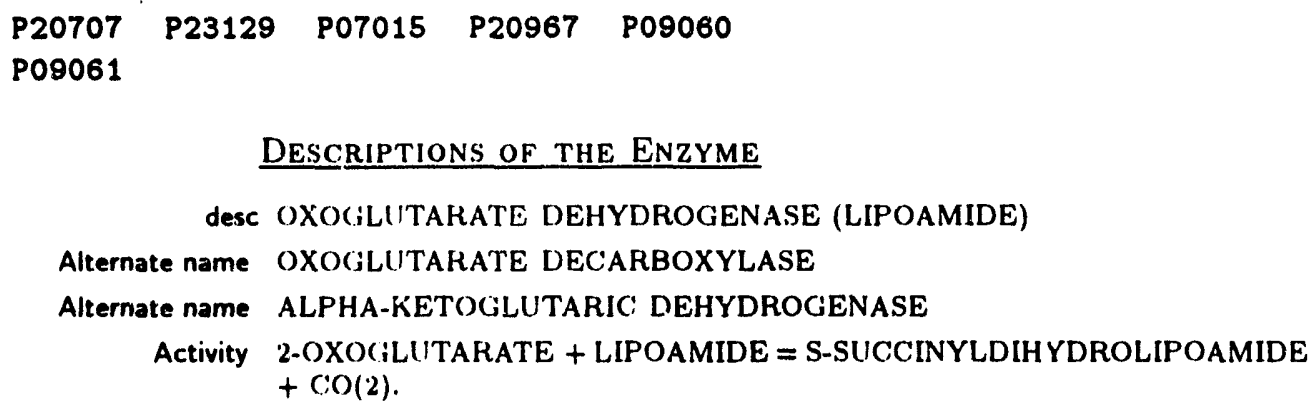

DESCRIPTIONS OF THE ENZYME

desc OXOGLLITARATE DEHYDROGENASE (LIPOAMIDE)

Alternate name OXOGLUTARATE DECARBOXYLASE

Alternate name ALPHA-KETOGLUTARIC: DEHYDROGENASE

Activity 2-OXO(iLUTARATE + LIPOAMIDE = S-SUCCINYLDIHYDROLIPOAMIDE $+\mathrm{CO}(2)$.

P07015 sucA 16.66768908 to 771709

\author{
Descriptions of the PEPTIDE \\ desc 2-OXOGLUTARATE DEHYDROGENASE E1 COMPONENT \\ (EC: 1.2.4.2) \\ E.coli gene SUICA
}

DESCRIPTIONS OF THE CDS

desc succinate requirement; al pha-ketoglutarate dehydrogenase (decarboxylase component)

Reaction: succinyl CoA_1

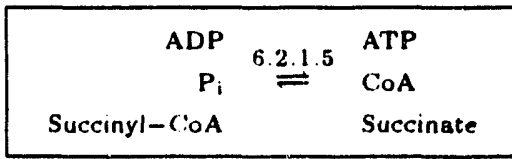

6.2.1.5: Succinate-CoA Ligase (ADP-Forming)

$\begin{array}{llll}\mathrm{P} 07460 & \mathrm{P} 25126 & \mathrm{P} 07459 & \mathrm{P} 09143\end{array}$ 


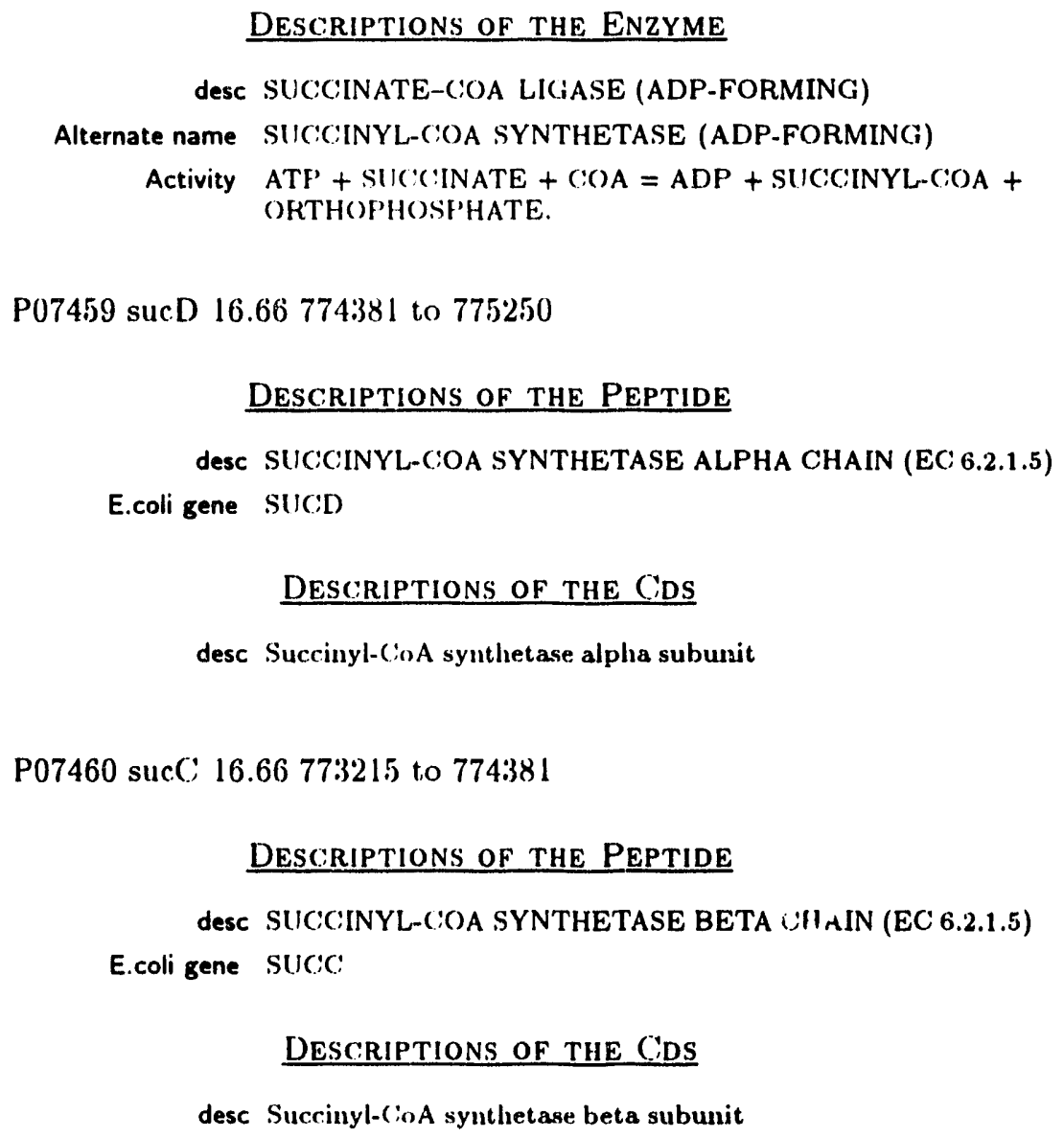

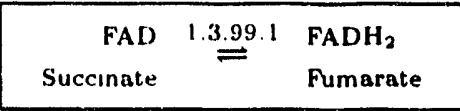

1.3.99.1: Succinate Dehydrogenase

$\begin{array}{lllll}\text { P08065 } & \text { P10444 } & \text { P08066 } & \text { P07014 } & \text { P08064 } \\ \text { P10446 } & \text { P00363 } & \text { P20922 } & \text { P17412 } & \text { P00364 } \\ \text { P20921 } & \text { P17596 } & \text { P17413 } & & \end{array}$

DESCRIPTIONS OF THE ENZYME

desc SUCCINATE DEHYDROGENASE 


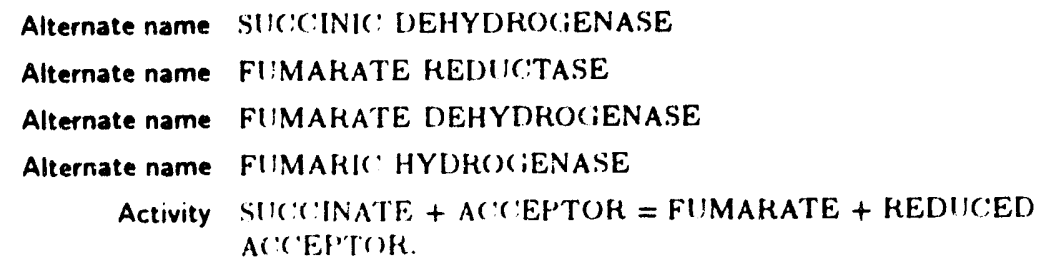

P00363 frdA 94.464412246 from 4414054 complement

DESCRIPTIONS OF THE PEPTIDE

desc FIIMAKATE REDIIC:TASE FLAVOPROTEIN SUBUNIT (EC 1.3.99.1)

E.coli gene FRDA

DESCRIPTIONS OF THE CDS

desc Fumarate reductase flavoprotein subunit

P00364 frdB 94.454411519 from 44122533 complement

\section{Descriptions of THE PEPTIDE}

desc FIIMARATE REDIJCTASE IRON-SULFUR PROTEIN (EC 1.3.99.1)

E.coligene FRISB

\section{DESCRIPTIONS OF THE CDS}

desc Fumarate reductase iron-sulfur protein subunit

P07014 sdhB 16.50767892 to $76 \times 60 \%$

Descriptions of the Peptide

derc SUC:CINATE DEHYDROGENASE IRON-SULFUR PROTEIN (EC 1.3.99.1)

E.coli gene SDHB

\section{DESC:RIPTIONS OF THE C.DS}

desc Succinate deliydrogenase; iron sulfur protein 
P10444 sdhA 16.50766110 to 767876

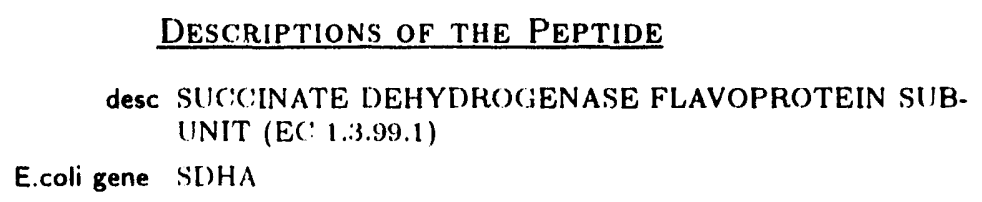

DEscriptions OF THE PePtide

desc SUCICINATE DEHYDROGENASE FLAVOPROTEIN SUBUNIT (EC: 1.3.99.1)

E.coli gene $S[) H A$

DESCRIPTIONS OF THE CDS

desc Succinate dehydrogenase; flavoprotein subunit

P10446 sdhC: 16.50765380 to 765769

Descriptions of the Peptide

desc SIIC:INATE DEHYDROGENASE CYTOCHROME B-556 SIIBINIT (EC: 1.3 .99 .1 )

E.coli gene SDHC:

\section{DESCRIPTIONS OF THE CDS}

desc succinate dehydrogenase; cytochrome b556

Reaction: fumarate_1

$$
\underset{\mathrm{H}_{2} \mathrm{O}}{\underset{\text { Fumarate }}{4.2 .1 .2} \mathrm{~L}-\mathrm{Mal}}
$$

4.2.1.2: Fumarate Dehydratase

$\begin{array}{llllll}\text { P00923 P14407 P05042 } & \text { P07343 } & \text { P07954 }\end{array}$

$\begin{array}{lll}\text { P10173 P14408 P08417 } & \text { P }\end{array}$

DESCRIPTIONS OF THE ENZYME

desc FUMARATE DEHYDRATASE

Alternate name FUMARASE

Activity L-MALATE = FUMARATE + H(2)O.

P00923 fumA 35.641695396 from 1697042 complement

DEscriptions of THE PEPTIDE 


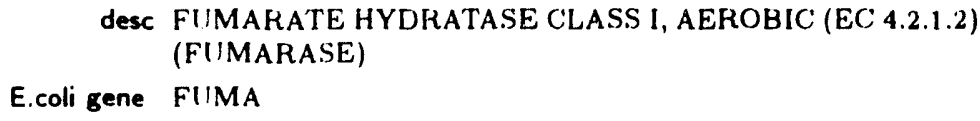

P05042 fumC: 35.64 169:385.3 from 1695256 complement

DESCRIPTIONS OF THE PEPTIDE

desc FUMARATE HYDRATASE CLASS II (EC 4.2.1.2)(FUMARASE) E.coli gene FUMC:

\section{DESCRIPTIONS OF THE CDS}

desc Fumarase

PI4407 fumB 93.45 4375992 from 4377638 complement

\section{Descriptions of the Peptide}

desc FUMARATE HYDRATASE CLASS I, ANAEROBIC (EC 4.2.1.2) (FIMARASE)

E.coli gene FUMB

\section{DESCRIPTIONS OF THE CDS}

desc Regulatory gene?

Reaction: malate_1

$$
\begin{aligned}
& \text { L-Mal } \stackrel{1.1 .37}{=} \mathrm{NADH}_{2} \\
& \text { NAD Oxalacetic Acid }
\end{aligned}
$$

1.1.1.37: Malate Dehydrogenase

$\begin{array}{lllll}\mathrm{P} 14152 & \mathrm{P} 11708 & \mathrm{P} 19446 & \mathrm{P} 22133 & \mathrm{P} 17783 \\ \mathrm{P} 08248 & \mathrm{P} 00346 & \mathrm{P} 04636 & \mathrm{P} 17505 & \mathrm{P} 19977 \\ \mathrm{P} 80040 & \mathrm{P} 80039 & \mathrm{P} 80038 & \mathrm{P} 06994 & \mathrm{P} 80037 \\ \mathrm{P} 19978 & \mathrm{P} 16142 & \mathrm{P} 19979 & \mathrm{P} 10887 & \mathrm{P} 19980 \\ \mathrm{P} 19981 & \mathrm{P} 25077 & \mathrm{P} 19982 & \mathrm{P} 19983 & \mathrm{P} 11386 \\ \mathrm{P} 10584 & & & & \end{array}$




\section{DESCRIPTIONS OF THE ENZYME \\ desc MALATE DEHYDROGENASE \\ Alternate name MALIC: DEHYDROC:IENASE \\ Activity L-MALATE + NAD $(+)=$ OXALOACETATE + NADH. \\ P06994 mdh 70.403402585 from 3403526 complement \\ DESCRIPTIONS OF THE PEPTIDE \\ desc MALATE DEHYDROGENASE (EC 1.1.1.37) \\ E.coli gerie $\mathrm{MDH}$ \\ DESCRIPTIONS OF THE CDS}

desc Malate dehydrogenase 


\section{Summary of the Pathway: THF-Synthesis_1}

\begin{tabular}{|rrl|}
\hline ADP & 1.5.1.5 & \\
Methylene-Tetrahydrofolate & 3.5 .4 .9 & ATP \\
NADP & 5.3 .4 & Formate \\
$\mathrm{P}_{\mathrm{j}}$ & & $\mathrm{NADPH}_{3}$ \\
$\mathrm{H}_{3} \mathrm{O}$ & $\mathrm{THF}$ \\
\hline
\end{tabular}

\section{Reaction: Methylene-Tetrahydrofolate_THF_1}

$$
\begin{array}{r}
\text { Methylene-Tetrahydrofolate } \stackrel{1.5 .1 .5}{=} \begin{array}{l}
\text { Methenyl-Tetrahydrololate } \\
\text { NADP }
\end{array} \\
\mathrm{NADPH}_{3}
\end{array}
$$

1.5.1.5: Methylenetetrahydrofolate Dediydrogenase (NADP+)

DESCRIPTIONS OF THE ENZYME

desc METHYLENETETIRAHYDROFOLATE DEIIYDROGENASE (NADP+)

Activity 5,10-METHYLENETETRAHYDROFOLATE + NADP $(+)=$ 5,10-ME'THENYLTETRAHYDROFOLATE + NADPH.

$$
\text { Pep??? cds??? Pos??? Loc??? }
$$

Reaction: Methenyl-Tetrahydrofolate_THF_1

$$
\text { Methenyl-Tetrahydrofolate } \stackrel{3.5 .4 .9}{=} 10-\text { Folmyl-TuF }
$$

3.5.4.9: Methenylletrahydrofolate Cyclohydrolase

\section{DESCRIPTIONS OF TIIE ENZYMIE}

desc METHENYLTETRAHYDROFOLATE CYCLOHYDROLASE Activity 5,10-METHYLENETETRAHYDROFOLATE $+\mathrm{H}(2) \mathrm{O}=10$ FORMYLTETRAHYDROFOLATE.

$$
\text { Pep??? cds??? Pos??? Loc??? }
$$




\section{Reaction: 10-For'myl-THF_THF_1}

$$
\begin{aligned}
& \begin{array}{r}
\text { 10-Formyl-THF } \\
\text { ADP } \stackrel{\text { 6.3.3.3 }}{=} \text { Formate }
\end{array} \\
& P_{i} \quad T H F
\end{aligned}
$$

6.3.4.3: Formate-Tetrahydrofolate Ligase

\section{DEscriptions OF TIIE ENZYME}

desc FORMATE-TETRAHYDROFOLATE LIGASE Alternate name FORMYLTETRAHYDROFOLATE SYNTHETASE

Activity ATP + OORMATE + TETRAHYDROFOLATE = ADP + ORTHOP,IOSPHATE + 10-FORMYLTETRAHYDROFOLATE.

Pep??? cds??? Pos??? Loc??? 


\section{Summary of the Pathway: Threonine-Synthesis_1}

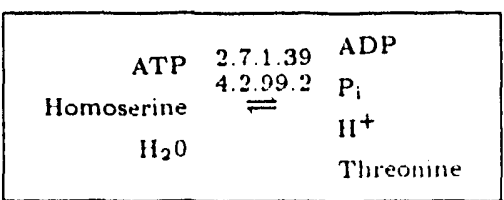

Reaction: Homo-SER_2

\begin{tabular}{rll|} 
ATP 2.7.1.39 & $\begin{array}{l}\text { ADP } \\
\text { O-Phospho-L-Homoserine } \\
\text { Homoserine }\end{array}$ & \begin{tabular}{l}
$\mathrm{H}^{+}$ \\
\hline
\end{tabular}
\end{tabular}

2.7.1.39: Homoserine Kinase $\begin{array}{lllll}\text { P04948 } & \text { P07128 } & \text { P08210 } & \text { P00547 } & \text { P04947 }\end{array}$

P17423

DESCRIPTIONS OF THE ENZYME

desc HOMOSERINE KINASE

Activity ATP + L-HOMOSERINE = ADP + O-PIOSPHO-L-HOMOSERINE.

P00547 thr B 0.002871 to 3800

Descriptions of tile Peptide

desc HOMOSERINE KINASE (EC 2.7.1.39) (HK)

E.coli gene THRB

Descriptions OF TIIE CDS

desc Homoserine kinase

Reaction: O-Phospho-L-HomoSerine_1

$$
\begin{array}{r}
\text { O-Phospho-L-Homoserine } \\
\mathrm{H}_{2} \mathrm{~A}
\end{array}
$$

4.2.99.2: Threonine Synthase

$\begin{array}{lllll}\text { P04990 P09123 P23669 P00934 P16120 } & \end{array}$ 


\section{DESCRIPTIONS OF THE ENZYME}

desc THREONINE SYNTHASE

Activity O-PHOSPHO-L-HOMOSERINE + H(2)O = L-THREONINE + ORTHOPHOSPHA'TE.

P009:34 thr(: 0.013801 to 5087

DESCRIPTIONS OF THE PEPTIDE

desc THREONINE SYNTHASE (EC 4.2.99.2)

\section{E.coli gene THRC:}

DESCRIPTIONS OF THE CDS

desc 'Threonine synthase 


\section{Summary of the Pathway: Tryptophan-Synthesis_1}

\begin{tabular}{|c|c|c|}
\hline 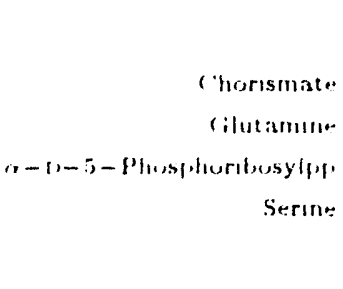 & $\begin{array}{llll}2 & 4 & 2 & 18 \\
4 & 1 & 1 & 48 \\
4 & 1 & 3 & 27 \\
4 & 2 & 1 & 201 \\
5 & 3 & 1 & 24\end{array}$ & 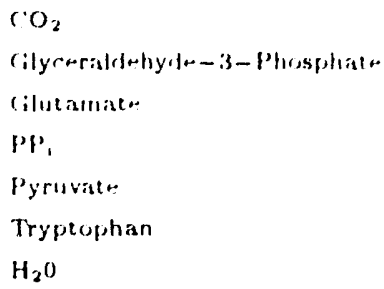 \\
\hline
\end{tabular}

Reaction: Chorismate_TRP_1

\begin{tabular}{|ll|}
\hline & Anthramlate \\
Chorismate 41327 & (ilutamato \\
& $\mathrm{H}^{+}$ \\
& Pyruviate \\
\hline
\end{tabular}

\begin{tabular}{|c|c|c|c|c|}
\hline P09785 & P09786 & P18483 & P05328 & P06531 \\
\hline P24773 & P25170 & P23315 & P18267 & P03963 \\
\hline P06557 & P00896 & P14953 & P00895 & P20463 \\
\hline P20580 & P20579 & P21689 & P15395 & P00898 \\
\hline P00897 & P21690 & P20170 & P05378 & P22099 \\
\hline P00899 & P00902 & P06558 & P14952 & P00904 \\
\hline P09575 & P20441 & P00908 & P20409 & P20576 \\
\hline P00901 & P00905 & P00900 & P00906 & P05379 \\
\hline P22101 & P00937 & & & \\
\hline
\end{tabular}

\section{DESCRIPTIONS OF THE ENZYME}

desC ANTHRANILATE SYNTHASE

Activity CHORISMATE + L-CiLUTAMINE = ANTHRANILATE + PYRUVATE + L-CILUTAMATE.

PO0X95 trpE 28.00 1328888 from 13330450 complement

DESCRIPTIONS OF THE PEPTIDE

desC ANTHRANILATE SYNTHASE (OMPONENT I (E(:1.1.3.2T)

E.soli gene TRPE 


\section{DESCRIPTIONS OF THE CDS}

desc anthranilate synthase

P(00904 trpD 28.00 1:3272933 from 1:328888 complement,

DESCRIPTIONS OF THE PEPTILE

desc ANTHRANILATE SYNTHASE COMPONENT II (EC: 4.1 .3 .27$)$ (CONTAINS: CILITTAMINE AMIDOTRANSFERASE; ANTHRANILATE PHOSPHORIBOSYLTRANSFERASE (E(:2.4.2.18)).

E.coli gene TRPD

\section{DESCRIPTIONS OF THE (IDS}

desc glutanine anidotrausferase-phosphoribosyl anthranilate transferase.

\section{Reaction: Anthranilate_TRP_1}

\begin{tabular}{|c|c|}
\hline $\begin{array}{r}\text { Anthranilate } 2.4 .2 .18 \\
\alpha-D-5-\text { Phosphoribosylpp }\end{array}$ & $\begin{array}{l}\mathrm{PP}_{\mathrm{i}} \\
N-\left(5^{\prime} \text {-Phosphoribosyl) - A.thranilate }\right.\end{array}$ \\
\hline
\end{tabular}

2.4.2.18: Anthranilate Phosphoribosyltransferase

$\begin{array}{lllll}\text { P00500 } & \text { P18261 } & \text { P03947 } & \text { P06559 } & \text { P12320 } \\ \text { P17170 } & \text { P20574 } & \text { P20575 } & \text { P12321 } & \text { P22096 } \\ \text { P07285 } & \text { P00904 } & \text { P00905 } & \text { P00906 } & \end{array}$

DESCRIPTIONS OF THE ENZYME

desc ANTHRANILATE PHOSPHORIBOSYLTRANSFERASE

Alternate name PHOSP'HRIBOSYL-ANTHRANILATE PYROPHOSPHORYLASE

Activity ANTHRANILATE + PHOSPHORIBOSYLPYROPHOSPHATE $=$ N-5'-PHOSPHORIBOSYL- ANTHRANILATE + PYROPHOSPHATE.

P00904 trpD 28.001327293 from 1328888 complement

DESCRIPTIONS OF THE PEPTIDE

desc ANTHRANILATE SYNTHASE COMPONENT II (E(: 4.1.3.27) (CONTAINS: GLUTAMINE AMIDOTRANSFERASE; ANTHRANILATE PHOSPHORIBOSYLTRANSFERASE (E( 2.4.2.18)). 
E.coli gene TRPD

DESCRIPTIONS OF THE CDS

desc glutamine amidotransferase-phosphoribosyl anthramilate: trans-

ferase

Reaction: 11-(5'-phosphoribosyl)-anthranilate_TRP_1

$N-\left(5^{\prime}-\right.$ Phosphoribosyl)-Anthranilate $\stackrel{5.3 .1 .24}{=} 1-(O-$ Carboxyphenylamino $)-1^{\prime}-$ Deoxyribulose $-5^{\prime}-P^{\prime}$

5.3.1.24: $N$-(5'-Phosphoribosyl)Anthranilate Isomerase

$\begin{array}{lllll}\mathrm{P} 24920 & \mathrm{P} 18483 & \mathrm{P} 05328 & \mathrm{P} 06531 & \mathrm{P} 06560 \\ \mathrm{P} 00909 & \mathrm{P} 24773 & \mathrm{P} 25170 & \mathrm{P} 00910 & \mathrm{P} 22098 \\ \mathrm{P} 16923 & \mathrm{P} 20167 & \mathrm{P} 12289 & \mathrm{P} 13997 & \mathrm{P} 17218 \\ \mathrm{P} 14718 & \mathrm{P} 00912 & \mathrm{P} 00908 & \mathrm{P} 20409 & \end{array}$

DESCRIPTIONS OF THE ENZYME

desc PHOSPHORIBOSYLANTHRANILATE ISOMERASE

Alternate name $\mathrm{N}-\left(5^{\prime}\right.$-PHOSPHORIBOSYL)ANTHRANILATE ISOMERASE

Alternate name PRAl

Activity N-(5-PHOSPHO-BETA-D-RIBOSYL)-ANTHR.ANILATE $=1$ (2-('ARBOXYPHENYLAMINO)-1- DEOXY-D-RIBULOSE 5PHOSPHATE.

P00909 trpe : 28.001325931 from 1327289 complement

Descriptions of the Peptide

desC INDOLE-3-GLYCEROL PHOSPHATE SYNTHASE (EC 4.1.1.48) (ICPS) / N-(5'-PHOSP'H()-RIBOSYL)ANTHRANILATE ISO)MERASE (EC 5.3.1.24) (PRAI).

E.coli gene TRPC:

DESCRIPTIONS OF THE CDS

desc $\mathrm{N}$-(5-phosphoribosyl)anthranilate isomerase indole-3-glycerolphosphate synthetase 
Reaction: 1-(o-carboxyphenylamine)-1'-deoxyribulose-5'-p_TRP_1

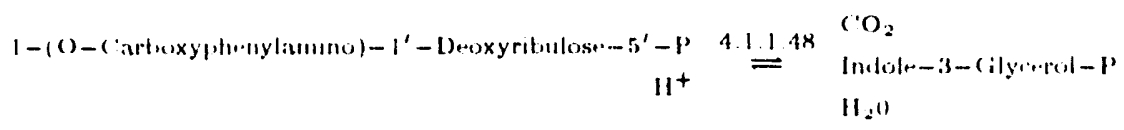

4.1.1.48: Indole-3-(ilyctrol Phosphate S'yuthase

$\begin{array}{lllll}\text { P24920 } & \text { P00911 } & \text { P18483 } & \text { P05328 } & \text { P06531 } \\ \text { P18268 } & \text { P03964 } & \text { P06560 } & \text { P00909 } & \text { P18304 } \\ \text { P17217 } & \text { P24773 } & \text { P25170 } & \text { P20577 } & \text { P20578 } \\ \text { P00910 } & \text { P22098 } & \text { P09575 } & \text { P00908 } & \text { P20409 } \\ \text { P00937 } & & & & \end{array}$

DESCRIPTIONS OF THE ENZYME

desc INDOLE-3-(ILY(:EKOL PHOSHHATE SYNTHASE

Activity 1-(2-(:ARBOXYPHENYLAMINO)-1-DEOXY-D)-RIBILOSE 5-P'HOSPHATE = 1-(INDOL-3- YL) ( ILYC:EROL :3-PHOSPHATE $+\mathrm{CO}(2)+\mathrm{H}(2) \mathrm{O}$.

P00909 trp(: 28.001325931 from $1: 327289$ complement

DESC:RIPTIONS OF THE PEPTIDE

desc INDOLE-3-(iLYC:EROL PHOSPHATE SYNTHASE (EO 4.1 .1 .48$)$ (ICI'S) / N-(5'-PHOSPIIO)-RIBOSYL)ANTHRANILA'TE ISO)MERASE (EC: 5.3.1.24) (['RAI).

E.coli gene TKPC:

DESCRIPTIONS OF THE (INS

desc N-(5-phosphoribosyl)anthranilate isomerase indole--3-glycerolphosplate: synthetase

Reaction: Indole-3-Glycerol-P_TRP_1

$$
\begin{aligned}
& \text { Indole-3-cilyrerol-P 4.2.1.20 Cilyceraldehyde-3-Phosphate } \\
& \text { Serine Tryptophan }
\end{aligned}
$$

4.2.1.20: Tryptophan Siynthase 


\begin{tabular}{|c|c|c|c|c|}
\hline 4671 & 5269 & P19867 & P07601 & P06 \\
\hline 122 & P00928 & P18284 & P00930 & \\
\hline 146 & P07344 & P11081 & P00929 & \\
\hline P220 & P16706 & P19868 & P07600 & 06 \\
\hline P12290 & P00932 & P18285 & P14552 & P17 \\
\hline 146 & P07345 & P1: & P00933 & 14 \\
\hline 2208 & P16578 & P13228 & P00931 & \\
\hline
\end{tabular}

DESCRIPTIONS OF THE ENZYME

desc TRYPTOPHAN SYNTHASE

Alternate name TRYPTOPHAN DESMOLASE

Activity L-SERINE + 1-(INDOL-3-YL)GLYCEROL 3-PHOSPHATE = L-TRYPTOPHAN + CLYCERALDEHYDE 3-P'HOSPHATE.

P00928 trpA 28.001323920 from 1324726 complement.

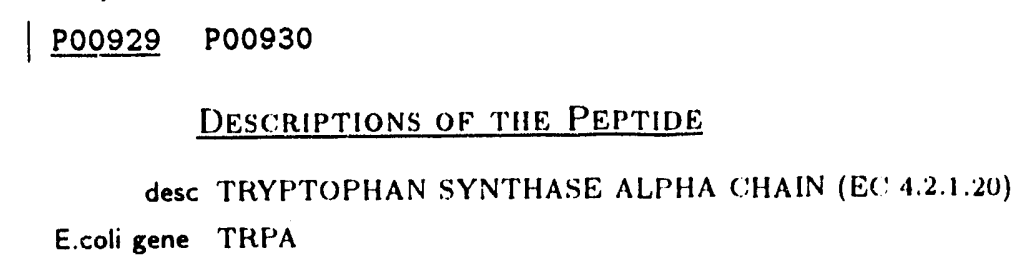

\section{DESCRIPTIONS OF THE CDS}

desc tryptoplian synthase $A$ protein

P00932 trpB 28.001324726 from 1325919 complement

DESCRIPTIONS OF THE PEPTIDE

desc TRYPTOPHAN SYNTHASE BETA (HAIN (E(: 4.2.1.20)

E.coli gene TRPB

DESCRIPTIONS OF THE C'DS

desc tryptophan synthase B protein 


\section{Summary of the Pathway: Tyrosine-Synthesis}

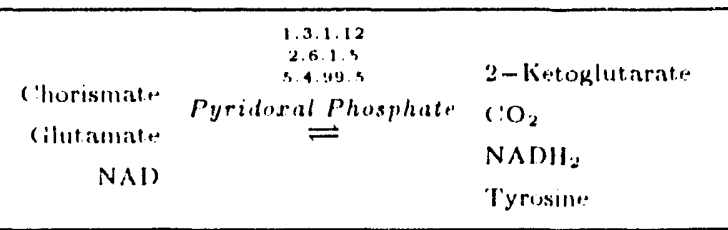

Reaction: Chorismate_1

$$
\text { (horismate } \stackrel{5.4 .99 .5}{=} \text { Prephenate }
$$

5.4.99.5: ('horismale Mulase

P19080 P07022 P21204 P07023

DESCRIPTIONS OF THE ENZYME

desc CHORISMATE MUTASE

Activity CHORISMATE $=$ PREPHENATE.

P07022 pheA 56.642750863 to 2752023

Descriptions of the Peptide

desc (HORISMATE MUTAASE (EC: 5.4.99.5) / PREPHENATE DEHYDRATASE (EC; 4.2.1.51).

E.coli gene PHEA

DESCRIPTIONS OF THE COSS

desc Chorismate mutase-P'-prephenate dehydratase

P07023 tyrA 56.642752066 from 2753187 complement

DESGRIPTIONS OF THE PEPTIDE

desc (HORISMATE MUTASE (EC: 5.4.99.5) / PREPHENATE DEHYDRO(.SENASE (EC: 1.3 .1 .12$)$.

E.coli gene TYRA

DESCRIPTIONS OF THE (IDS 
desc ('horismate mutase $T$-prephenate dehydrogenise

Reaction: Prephenate-TYR_1

$$
\begin{aligned}
\text { NAl } 13.12 & \mathrm{CO}_{2} \\
\text { Prephennte } & \mathrm{NAOH}_{2} \\
& \mathrm{P}-\mathrm{Hydroxy}-\text { Phenylpyruvate }
\end{aligned}
$$

1.3.1.12: Prephenate Dehydrogenase

P20692 P07023

DESCRIPTIONS OF THE ENZYME

desc PREPHENATE DEHYDRO(iENASE

Activity PREPHENATE + NAD $(+)=4-H Y L$ HOXYPIENYLPYRIIVATE $+C O(2)+\mathrm{NADH}$.

P0702:3 tyrA 56.642752066 from 2753187 complement

Descriptions of the Peptide

desc (HORISMATE MUTASE (EC: 5.4.99.5) / PREPHENATE DEHYDRO(:ENASE (EO 1.3.1.12).

E.coligene TYRA

DESCRIPTIONS OF THE CINS

desc ('horismate mutase $T$-prephenate dehydrogenase

\begin{tabular}{|c|c|c|}
\hline & 2.5 .1 .5 & \\
\hline $\begin{array}{l}\text { Cilutamate } \\
\text { P-Hydroxj - Phenylpyruvate }\end{array}$ & Pyridoxai Phosphate & $\begin{array}{l}2 \text { - Ketoglutarate } \\
\text { Tyrosine }\end{array}$ \\
\hline
\end{tabular}

Reaction: P-hydroxy-PhenylPyruvate_TYR_.1

2.6.1.5: Tyrosine Aminotransferase

DESCRIPTIONS OF THE ENZYME

desc TYROSINE AMINOTRANSFERASE

Alternate name TYROSINE THANSAMINASE 
Activity L-TYROSINE + 2-OXOCILUTARATE = 4-HYDROXYIHENYLPYRIIVATE + L.(iLUTAMATE.

Pep???? tyrB 91.984296866 to 4298059

DESCRIPTIONS OF THE (DIS

desc Tyrosine aninotransforase; tyrosine repressible 


\section{Summary of the Pathway: Valine-Synthesis_1}

\begin{tabular}{|c|c|c|}
\hline 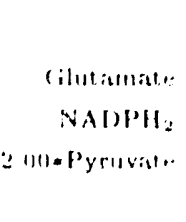 & $\begin{array}{cccc}1 & 1 & 1 & 86 \\
2 & 6 & 1 & 42 \\
4 & 1 & 3 & 18 \\
4 & 2 & 1 & 9 \\
& \end{array}$ & 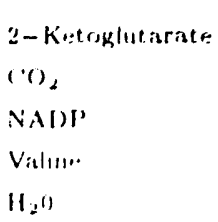 \\
\hline
\end{tabular}

Reartion: Pyruvate_VAL_1

$$
200 \text { Pyruvale } \stackrel{413182-A c e t c l a c t i s t e}{=}
$$

1.1.3.18: Arrlolartaw Siynthase

$\begin{array}{lllll}\text { P09342 } & \text { P } 17597 & \text { P14874 } & \text { P08142 } & \text { P09114 } \\ \text { P07342 } & \text { P00892 } & \text { P00894 } & \text { P21622 } & \text { P00893 } \\ \text { P13048 } & \text { P08143 } & & & \end{array}$

DESCRIPTIONS OF THE ENZYME

desc ACETOLACTATE SYNTHASE

Activity 2 -AC:ETOLACTATE $+(C)(2)=2$ PYRIIVATE.

P(0)(962) ilv( : $\times 4.90) 3997 \times 469$ to 39980115

DESCRIPTIONS OF THE PEPTIUE

desC ACETOLAC'TATE SYYNTHASE (EC : 4.1.3.18) ISCOYYME II, LARCIE SUHIINIT (AHAS- II) (ACETOHYDROXY-AC:ID) SYNTHASE II).

E.coli gene IIVC:

\section{DESCRIPTIONS OF THE ('DS}

desc Aretohydroxy arid synthase II ; valine-insensitive large suth unit

$P(0) \times 9.3$ ilv1 $1.8 .3 \times 5, \times 97$ to 87618

Deschiptions of the Peptide 


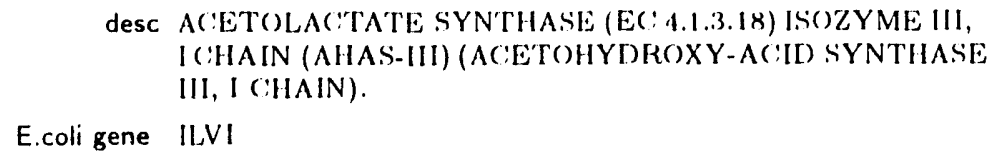
I (HAIN (AHAS-III) (ACETOHYDROXY-AC:ID) SYNTHASE III, I CHAIN).

E.coli gene IILVI

DESCRRIPTIONS OF THE (IUS

desc Aretohydroxy acid synthase III : valine-sensitive large: subunit

P00894 ilvH $1.83 \times 7621$ to 88112

DESCRIPTIONS OF THE PEPTIDE

desc AC:ETOLAC:TATE SYNTHASE (E(:4.1.3.18) ISOZYME III, H CHAIN (AHAS-III) (ACETOHYDOROXY-AC:II) SIYNTHASL: III, H ('HAIN).

E.coli gene IIVVH

\section{DESCRIPTIONS OF TIIE (IDS}

desc Acetolydroxy acid synthase III ; valine-sensitive small subunit

P08142 ilvB 82.69 3875291 from 3876979 complement.

Desciriptions of the Peptide

desc A(:ETOLAC:TATE SYYNTIASE (EC: 4.1.3.18) IS()ZYME I, LARC:ESUBUINIT (AHAS-I) (ACETOHYDROXY-AC:ID) SIYNTHASE ().

E.coli gene IIJVB

\section{DESCRIPTIONS OF TIIE (INS}

desc Acetohydroxy acid synthase I valine-sensitive large subunit

P08143 ilvN 82.693874997 from 3875287 complement

DESCRIPTIONS OF THE PEPTIDE

desc ACETOLAC:TATE SYNTHASE (E(: 4.1.3.18) ISOZYME I, SMALL SUBIINIT (AHAS-I) (ACETOHYDROXY-A('II) SYYNTHASE I).

E.coli gene ILVN 


\section{DESCRIPTIONS OF THE CIDS}

desc Aretohydroxy acid synthase $X$; valine-sensitive small subunit

Pl:3048 ilvM $\times 4.9(0) 398(0115$ to 339803375

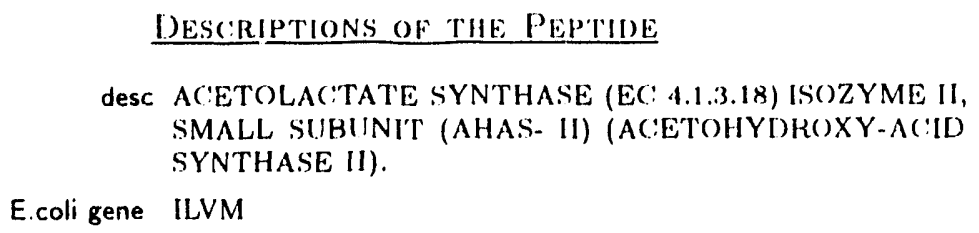

DESCRIPTIONS OF THE PEPTIDE

desc ACETOLAC:TATE SYNTHASE (EC: 4.1.3.18) ISOZYME II, SMALL SUBIINIT (AHAS- II) (AC:ETOHYDROXY-AC:ID SYNTHASE (I).

E.coli gene ILVM

\section{DESCRIPTIONS OF THE (IDS}

desc Aretohydroxy acid synthase II ; valine-insensitive small sulbilinit

Reaction: 2-aceto-Lactate_VAL_1

$$
\begin{aligned}
& 2 \text {-Acetolartate 1.1.1.86 } \alpha, \beta \text {-DiHydroxy-IsoValerate } \\
& \mathrm{NADPH}_{2} \text { NADP }
\end{aligned}
$$

\subsubsection{6: Ketol-Acid Reductoisomerase}

$\begin{array}{lll}\mathrm{P} 06168 & \mathrm{P} 05793 & \mathrm{P} 05989\end{array}$

DESCRIPTIONS OF THE ENZYME

desc KETOL-ACID REDIICTOISOMERASE

Alternate name DIHYDROXYISOVALERATE DEHYDRO)(IENASE (ISOMER$\operatorname{IZIN(i)}$

Alternate name AC:ETOHYDROXY ACIID ISOMEROREDICTASE

Activity 2,3-DIHYDROXYISOVALERATE + NADP $(+)=2$-ACETOLACTATE $+\mathrm{NADPH}$.

P05793 ilv(: 84.913985878 to 398735.3

\section{DESCRIPTIONS OF THE PEPTIDE}

desc KETOL-ACID REDUITTOISOMERASE (EC:1.1.1.86) (AC:ETOHYDHOXYACID ISOMER()REDUIC:TASE).

E.coli gene ILVC' 


\section{DESCRIPTIONS OF THE CINS}

desc ketol-acid reductuisomerase

Ruaction: alpha,heta-Dihydroxy-Isovalerate_VA L_1

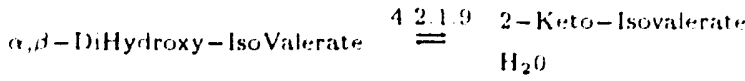

4.2.1.9: Dihydrox:y-Acid Dehydratase

P05791

DESCRIPTIONS OF THE ENZYME

desc DIHYDROXY-A(II) [OEHYDRATASE

Activity 2,3-D)IHYDR()XY-3-METHYLBITTAN()ATE = 2-()X()-3-METIIYLBIITAN()ATE $+H(2) 0$.

P(05791 ilvD $\times 4.913981389$ to 398.3236

DEsC:RIPTIONS OF THE PEPTIDE

desc DIHYDROXY-ACID DEHYDRATASE (E(:4.2.1.9)

E.coli gene (LVI.)

DESCRIPTIONS OF THE (IISS

desc dihydroxyacid deliydratase

Reaction: 2-keto-Isovalerate_VAL_1

2-Keto-Isovalerate 2.6.1.42 2-Ketoglutarate

Glutamate Valine

2.6.1.42: Branched-C'hain Amino Acid Aminotransferase

P00510 P15168

DESCRIPTIONS OF THE ENZYME

desC HRAN(HED)-(HAIN AMINO ACII) AMINOTRANSFERASE 
Alternate name TRANSAMINASE B

Activity L-LEU(:INE + 2-0X()(iLUTARATE = 4-METHYL-2-()XOI'ENTANOATE + L-CILUTAMATE.

P(00510 ilve $\times 4.903398(0) 3955$ to 39813324

DESCRIPTIONS OF THE PEPTIDE

desc BRANCHED-('HAIN AMINO ACID AMINO'TRANSFERASE (EC: 2.6.1.42) (TRANSAMINASE B).

E.coli gene ILVE

DESCRIPTIONS OF THE ('DS

desc branched-chain amino-arid aminotransforass? 


\section{Sumnnary of the Pathway: Valine-Synthesis_2}

\begin{tabular}{|c|c|c|}
\hline 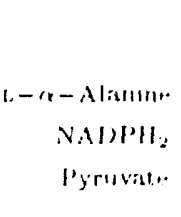 & $\begin{array}{c}11.166 \\
2.6 .166 \\
1.1318 \\
421.9 \\
\text { Pyratuato } \\
=\end{array}$ & $\begin{array}{l}\mathrm{CO}_{2} \\
\mathrm{NAll} H \\
\mathrm{Valll} . \\
\mathrm{H}_{2} \mathrm{O}\end{array}$ \\
\hline
\end{tabular}

Reaction: Pyruvate_VAL_1

$$
200 \text { Pyruvate } \stackrel{4.1318}{=} \begin{aligned}
& 2-\text { Aretoliactate } \\
& 10.2
\end{aligned}
$$

4.1.3.18: Acrtolnetalt Signthase

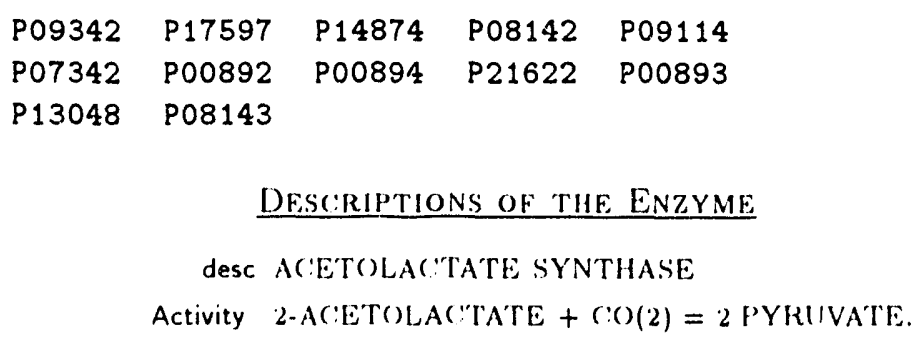

DESCRIPTIONS OF THE ENZYME

desc A(ETOLACTATE SYNTHASE

Activity 2 -ACETOLAC 'TA'TE + $\because O(2)=2$ PYRIIVATE.

P008992 ilv(: $\times 4.90) 3997 \times 169$ too $393 \times(0) 115$

DESC:RIPTIONS OF THE PEPTIUE

desC ACETOLACTATE SYNTHASE (ES: 4.1.3.18) ISC)ZYME II, LARCIE SUBHINIT (AHAS- H) (ACETOHYDRO)XY-ACII) SYNTHASE II).

E.coli gene ILVC;

DESCRIPTIONS OF THE (:DS

desc Aretoliydroxy acid synthase II ; valine-insonsitive large: subunit

Po(0)893 ilv1 1.8:3 85897 to 87618

DESCRIPTIONS OF THE PEPTINE 
desc ACETOLAC'TATE SYNTHASE (EC:4.1.3.18) ISOZYME III, I CHAIN (AHAS-III) (ACETOHYDROXY-AC'II) SYNTHASE III, I (HHAIN).

E.coli gene ILVI

\section{DESCRIPTIONS OF THE ('INS}

desc Acetohydroxy acid synthase III ; valine-sensitive large subunit

P00894 ilvH 1.8387621 to 88112

DESCRIPTIONS OF THE PEPTIDE

desc AC:ETOLAC:TATE SYNTHASE (E(; 4.1.3.18) ISOZYME II, H CHAIN (AHAS-II) (ACETOHYDROXY-AC:II) SYNTHASE III, H ('HAIN).

E.coli gene ILVH

DESCRIPTIONS OF THE ('DS

desc Acetohydroxy acid synthase III ; valine-sensitive small subunit

P08142 ilvB $\$ 2.693875291$ from 3876979 complement

DESCRIPTIONS OF THE PEPTIDE

desC ACETOLACTATE SYNTHASE (EC 4.1.3.18) ISOZYME I, LARCIE SUBIINIT (AHAS-I) (ACETOHYDROXY-ACIID) SYNTHASE I).

E.coligene ILVB

DESS:RIPTIONS OF THE CINS

desc Acetoliydroxy acid synthase I valine-sensitive large subunit

P081433 ilvN 82.693874997 from 3875287 complement

DESCRIPTIONS OF THE PEPTIDE

desc ACETOLACTATE SYNTHASE (EC: 4.1.3.18) ISOZYME I, SMALL SUBUNIT (AHAS-I) (ACETOHYDROXY-ACII) SYNTHASE ().

E.coli gene ILVN 


\section{DESCRIPTIONS OF THE (IDS}

desc Acetohydroxy acid synthase $X$; valine-sensitive small subunit

P'13(148 ilviv $\times 4.9033980(115$ to $339 \times(3) 375$

\section{DESCRIPTIONS OF THE PEPTIINE}

desc ACETOLAC'TATE SYNTHASE (EC 4.1 .3 .18 ) ISOZZYME II, SMALL SUBUNIT (AHAS- II) (ACETOHYDROXY-AC:IL) SYNTHASE (I).

E.coligene ILVM

\section{DESCRIPTIONS OF THE ('DS}

desc Aretuhydroxy acid synthase II ; valinte-insensitive small subunit

Reaction: 2-aceto-Lactate_VAL_1

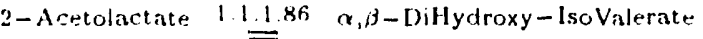

1.1.1.86: Ketol-Acid Reducloisomerase

$\begin{array}{lll}\text { P06168 P05793 P05989 } & \text { P }\end{array}$

DESCRIPTIONS OF THE ENZYME

desc KETOL-AC:ID REDUC:TOISOMERASE

Alternate name DIHYDROXYISOVALERATE DEHYDKO)(IENASE (ISOMERIZIN(i)

Alternate name A(ETOHYI)KOXY A(II) ISOMEROREDU(ITASE

Activity 2,3-DIHYLROXYISOVALERATE + NADP $(+)=2-A($ ETOLACTATE + NADPH.

P05793 ilv(: 84.9139855878 to 39873533

DEsc:RIPTIONS OF THE PEPTIDE

desc KETOL-ACID REDUC'TOISOMERASE (E(:1.1.1.86) (ACETOHYDROXY. A(:ID ISOMEROREDU(ITASE).

E.coli gene ILVC: 


\section{DESCRIPTIONS OF THE (DS}

desc ketol-acid reductoisomerase

Reaction: alpha,beta-Dihydroxy-Isovalerate_VAL_1

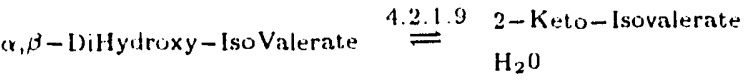

\subsubsection{9: Dihydroxy-Acid Dehydratase}

P05791

\section{DESCRIPTIONS OF THE ENZYME}

desc DIHYDROXY-ACID DEHYDRATASE

Activity 2,3-DIHYDROXY-3-METHYLBUTANOATE $=$ 2-()X()-3-METHYLBITANOATE $+\mathrm{H}(2) \mathrm{O}$.

P05791 ilvD 84.913981389 to 3983236

DESCRIPTIONS OF THE PEPTIDE

desC DIHYDROXY-ACID DEHYDRATASE (EC: 1.2.1.9)

E.coli gene ILVD

\section{DESCRIPTIONS OF THE ('DS}

desc dihydroxyacid dehyrdratase

Reaction: 2-keto-Isovalerate_VAL_2

$$
\text { 2-Keto-Isovalerate } 2.6 .1 .66 \text { Pyruvate }
$$

$$
\mathrm{L}-\alpha-\text { Alanine } \quad \text { Valine }
$$

2.6.1.66: Valine-Pyruvate Aminotransferase.

P09053

DESCRIPTIONS OF THE ENZYME

desc VALINE-PYRUVATE AMINOTRANSFERASE 


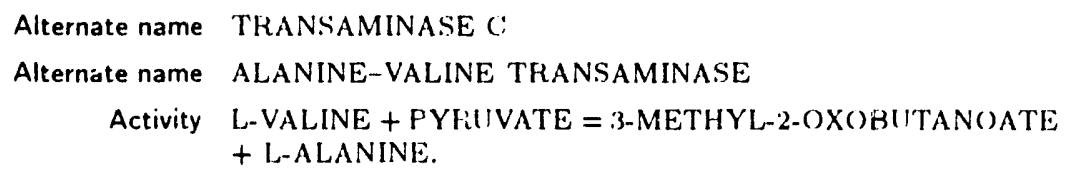

PO(9)53 avt A 84.5137633602 from 3764282 complement.

\section{DEscriptions of the PePtide}

desc VALINE-PYRUIVATE AMINO'TRANSFERASE (EC: 2.ti.1.ti6) (TRANSAMINASE () (ALANINE-VALINE TRANSAMINASE).

E.coli gene AVTA

\section{DESCRIPTIONS OF THE ('DSS}

desc Alanine-ialpha-ketoisovalerate transaminase; transinninisse: 


\section{Miscellaneous Reactions}

Reaction: Alanine-Synthesis_1

$\begin{array}{ll}\text { (ilutamate: Pyridoxal Phosphate } & 2-\text { Ketoglutarate } \\ \text { Pyruvate } & \stackrel{2-\alpha-A l a n i n e}{\rightleftharpoons}\end{array}$

2.6.1.2: Alanine Aminotransferase

DESCRIPTIONS OF THE ENZYME
desc ALANINE AMINOTRANSFERASE
Alternate name (iLITAMIC-PYYIVIC: TRANSAMINASE
Alternate name (iLITAMIC-ALANINE TRANSAMINASE
Activity L-ALANINE + 2-OXOCILITARATE = PYRIIVATE + L-
(iLUTAMATE.

Pep???? cds???? Pos???? Loc????

Reaction: Creatine_Creatine_1

$\begin{aligned} & \text { ATP } 2.7 .3 .2 \begin{array}{l}\text { ADP } \\ \text { Creatine-Phosphate } \\ \mathrm{H}^{+}\end{array} \\ &\end{aligned}$

2.7.3.2: C'reatine hinase

DESCRIPTIONS OF THE ENZYME

desc ('REATINE KINASE

Activity ATP + CREATINE = ADP + PHOSPHOCREATINE.

Pep??? cds??? Pos??? Loc???

Reaction: Creatine_Creatine 2

Creatine $\stackrel{3.5 .2 .10}{=} \begin{aligned} & \text { Creatinine } \\ & \mathrm{H}_{2} 0\end{aligned}$

3.5.2.10: (realininase 


$$
\begin{aligned}
& \text { DESC:RIPTIONS OF THE ENZYME } \\
& \text { desc (REATININASE } \\
& \text { Alternate name (KEATININE AMIDOHYI)R()LASE } \\
& \text { Activity ('REATININE + H(') })=(\text { REATINE. }
\end{aligned}
$$

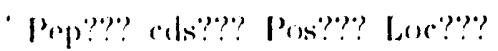

Reaction: GLY_GLY_1

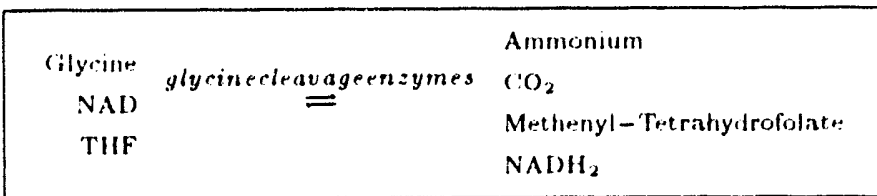

glycine rleavage enzymes

Pep"??? cols??"? Pos???? Loc???

Reaction: Glycine-Synthesis_1

$$
\begin{array}{cl}
\text { Serine } 212.1 & \begin{array}{l}
\text { Glyoune } \\
\text { THF }
\end{array} \\
& \text { Methenyl-Tetrahydrofolate } \\
\mathrm{H}_{2} \mathrm{O}
\end{array}
$$

2.1.2.1: Cilyeine Hydroxymethyltransferase

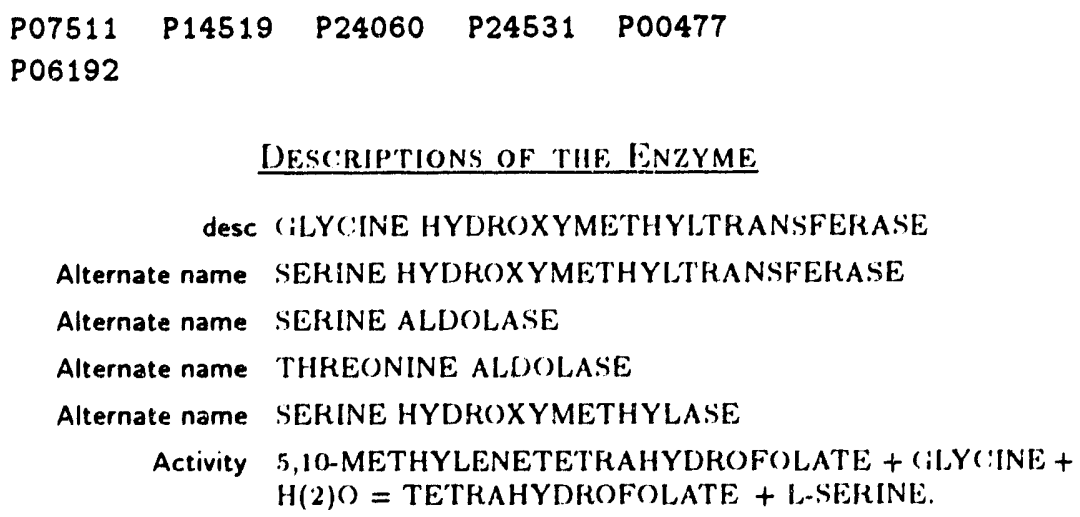

DESCRIPTIONS OF THE, ENZYME

desc (ILY(:INE HYDROXYMETHYLTRANSFERASE

Alternate name SERINE HYDKOXYMETHYLTRANSFERASE

Alternate name SERINE ALDOLASE

Alternate name THREONINE ALDOLASE

Alternate name SERINE HYDROXYMETHYLASE

Activity 5,10-METHYLENETETRAHYDROFOLATE + (iLYC:INE + $H(2) O=$ TE'TRAHYDROFOLATE + L-SERINE.

P(0)(0477 gly $54.8(0) 26937655$ from 2695018 complement 


\section{DESCRIPTIONS of THE PEPTIDE \\ desc SERINE HYDROXYMETHYLTRANSFERASE (E) : 2.1 .2 .1 ) (SERINE METHYLASE) (SHMT). \\ E.coligene (iLYA}

DESCRIPTIONS OF THE (UNS

desc Serine hydroxymethyltransferase:

\section{Reaction: L-Asparagine-Synthesis_1}

\begin{tabular}{rll|}
\hline ATP & AMP \\
Cilutamme & i354 Asparagine \\
L-Aspartate & (ilutamate \\
& $\mathrm{PP}_{\mathrm{i}}$ \\
& $\mathrm{H}^{+}$ \\
\hline
\end{tabular}

6.3.5.4: Asparagine S'ynthase (Cilutamine-Hydrolysing)

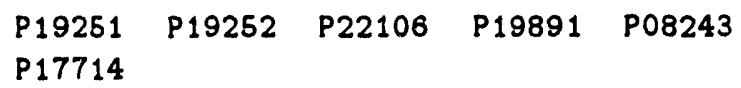

\section{DESCRIPTIONS OF THE ENZYME}

deSC ASPARAGINE SYNTHASE ((ILITAMINE-HYDROLYSING)

Alternate name ASF'ARACINESYNTHETASE ((ILUTAMINE-HYDROLYSSIN(i)

Activity ATP + L-ASP'ARTATE + L-(iLIITAMINE = AMI + PY. ROPHOSPHATE + L-ASPARACINE + L-CILIITAMATE.

P22106 asnB 15.5\% 707750 from 709414 complement

| P19251 P19252

Descriptions of the Peptide

desc ASPAHAGINE SYNTHETASE B ((ILIITAMINE-HYDROLYZIN(i) (EC. 6.3.5.4)

E.coli gene ASNB

DESCRIPTIONS OF THE COS

desc Asparagine synthetase $B$ 
Reaction: L-Asparagine-Synthesis_2

$\begin{array}{rll}\text { Ammonia } & \text { 6.3.1 I } & \text { AMP } \\ \text { ATP } & = & \text { Asparagio.. } \\ \text { L-Aspitrtit. } & & P P_{1}\end{array}$

6.3.1.1: Aspartate dmmonia Ligasp

P00963

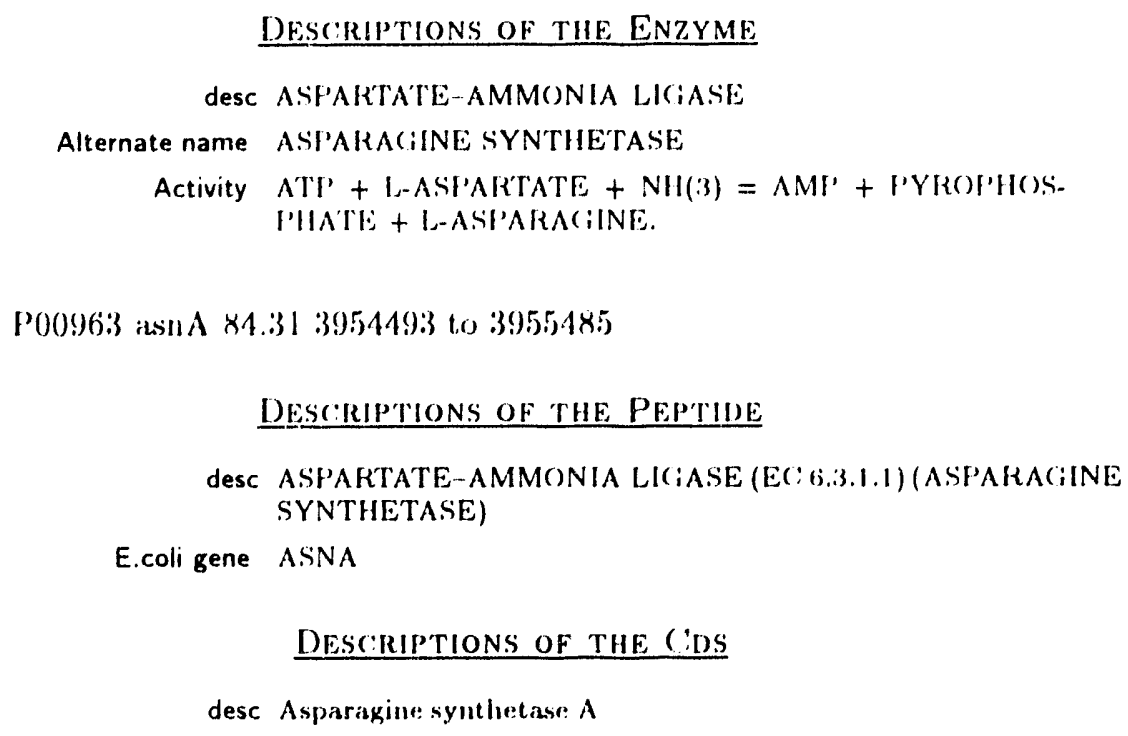

DESCRIPTIONS OF THE ENZYME

desc ASF'ARTATE-AMMONIA LIGIASE

Alternate name ASPARACINE SYNTHETASE

Activity ATP + L-ASP'ARTATE + NH(3) = AMP' + PYROPHOSSPIIATE + L-ASPARACINE.

P009633 asnd $\times 4.313954493$ to 3955,4855

DESCRIPTIONS OF THE PEPTIDE

desc ASPARTATE-AMMONIA LICIASE (EC;6.3.1.1) (ASPARACINE SYNTHETASE)

E.coli gene ASNA

DESCRIPTIONS OF THE (DS

desc Asparagines synthetase A

Reaction: L-Asparagine-Synthesis_3

$\begin{aligned} \text { Ammonia } & \text { ATSP } \\ \text { ATP } & = \\ \text { Aspartate } & \mathrm{P}_{1}\end{aligned}$

6.3.1.4: Aspartale Ammonia Ligase (ADP-Forming)

DESCRIPTIONS OF THE ENZYME

desc ASPAKTATE-AMMONIA LKIAASE (ADP-FOKMIN(i)

Alternate name ASPARACINE SYNTHETASE (ADP-F(NMING(i)

Activity ATP' + L-ASP'ARTATE + NH(3) $=$ ALP + P'YROPHOSS-

PHATE + L-ASP'ARAGINE. 
Pep???? chls?"?" Pos?"?"? Loc???"?

Reaction: L-Aspartate-Synthesis_1

\begin{tabular}{|c|c|c|}
\hline & 2.6111 & \\
\hline Gilutamate & Pyridoral Phosphaste & $2-k e l s g l u t a r a t .$. \\
\hline Oxaliwetce Acol & & 1.-Aspartittr: \\
\hline
\end{tabular}

2.6.1.1: Aspartate Aminolransferase

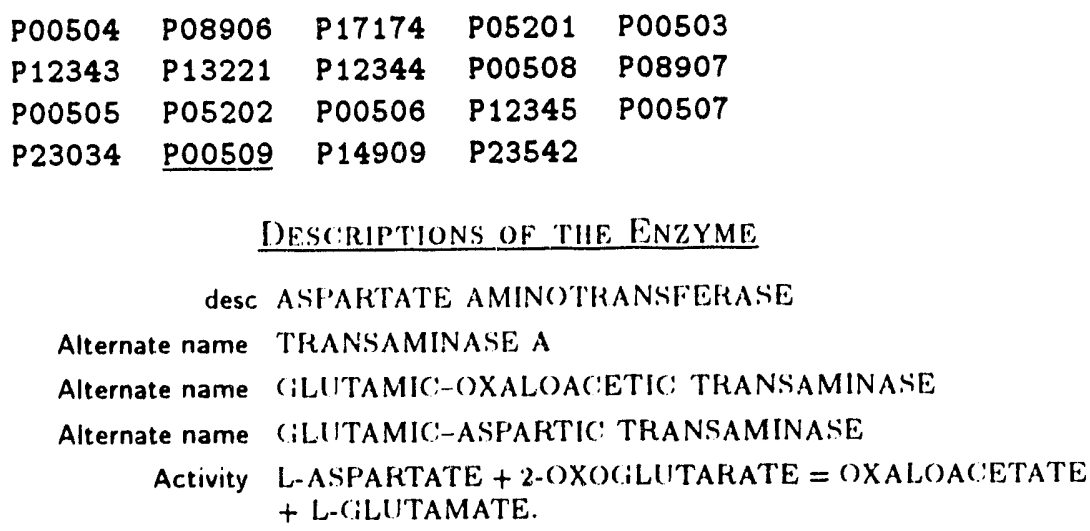

DESC:RIPTIONS OF THE ENZYME

dese ASPARTATE AMINOTHANSFERASE

Alternate name TRANSAMINASE A

Alternate name (iLITTAMIC:-OXALOACETIC: TRANSAMINASE

Alternate name (iLUTAMIC-ASPARTIC: TRANSAMINASE

Activity L-ASPARTATE + 2-OXOCiLUTARATE = OXALOACETATE

+ L-CiLUTAMATE.

P00509 asp(: 20.78 991199 from 992389 complement.

Descimptions of the Peptide

desc ASTAR'TATE AMINO'TRANSFERASE (EC:2.6.1.1)('THANSAMINASE A)

E.coli gene ASI'

DESC:RIPTIONS OF THE ('I)S

desc Aspartate aminotransferase

Reaction: NADPH2_1

$$
\begin{aligned}
& \mathrm{NAD} \stackrel{1.6 .1 .1 \mathrm{NADH}_{2}}{=} \\
& \mathrm{NADPH}_{3} \rightleftharpoons \mathrm{NADP}
\end{aligned}
$$

1.6.1.1: NAD(P)+ Transhydrogenase (B-Siperific)

P11024 P07001 P07002 


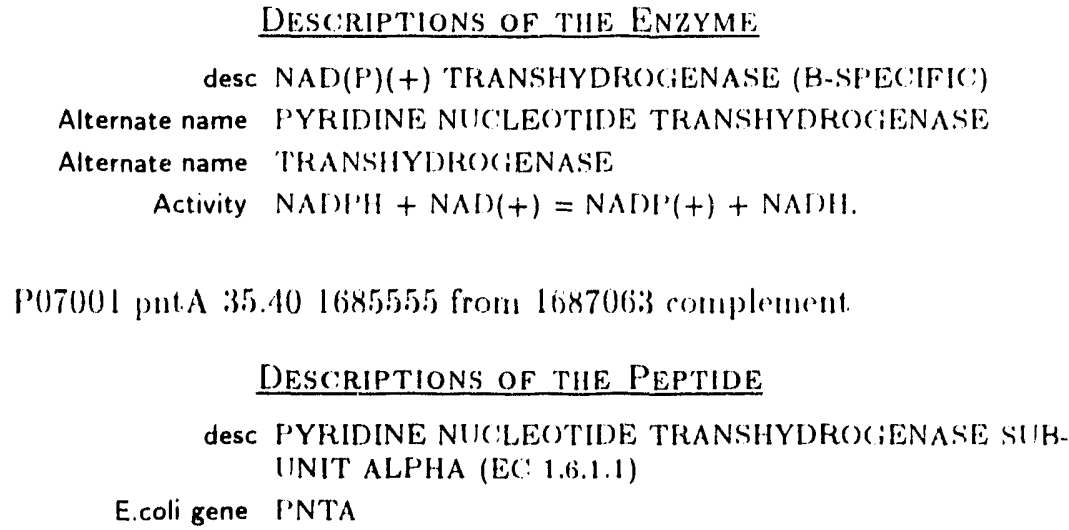

DESCRIPTIONS OF THE ENZYME

desc NAD(P)(+) TRANSHYDROCIENASE (B-SPECIFIC:)

Alternate name PYRIDINE NUCLEOTIDE TRANSHYDROCIENASE

Alternate name TRANSHYDRO)(IENASE

Activity $\mathrm{NADH}+\mathrm{NAD}(+)=\mathrm{NADP}(+)+\mathrm{NADH}$.

P07001 pHtA 35.40 16855555) from l6870633 complement.

\section{DESCRIPTIONS OF THE PEPTIDE}

desc PYRIDINE NIU(LEOTIDE TRANSHYDRO(IENASE SUH IINIT ALPHA (E: 1.6.1.1)

E.coli gene PNTA

DESCRIPTIONS OF THE CDS

desc Pyridine nucleotide transhydrogenase alpha subunit

P07002 put.B 35.40 16841336 from 16855524 complement.

DESC:RIPTIONS OF THE PEPTIDE

desc PYRIDINE NUILEOTIDE TRANSHYDROCIENASE SUHIINIT BETA (EC: 1.6.1.1)

E.coligene P'NTB

DESC:RIPTIONS OF THE CINS

desc Pyrilline mucleotide transhydrogenase Beta sulumit

Reartion: NADP_NAD_1

$$
\begin{gathered}
\mathrm{NAOP} \\
\mathrm{H}_{2} \mathrm{O}
\end{gathered}
$$

\section{1 .2 .00}

Pep??? cds??? Pos??? Loc????

Reaction: NAD_NAD_1

$$
\begin{array}{rll}
\mathrm{NACD} & 3.6 .1 .22 & \mathrm{AMP} \\
\mathrm{H}_{2} \mathrm{O} & = & \text { Nirotinamide Nucleotide }
\end{array}
$$

3.6.1.22: NAD $)^{+}$l'yrophosphatase 


$$
\begin{aligned}
& \text { DESC:RIP'TIONS OF THE ENZYME } \\
& \text { desc NAD+ PYROPHOSPHATASE } \\
& \text { Activity NAD }(+)+H(2)()=\text { AMP + NMN. }
\end{aligned}
$$

Pep???? rds???? Pos???"? Loc???

Reaction: NAD_NAD_2

NAI) 3.2.2.5 Adenosine Diphosphate Ribose

$\mathrm{H}_{2} \mathrm{O}$ Niacinamide

3.2.2.5: $N A D^{+}$Nucleosidase

DESCRIPTIONS OF THE ENZYME

desc NAD)(+) NIICLEOSIDASE

Alternate name NADASE

Alternate name DPNASE

Alternate name DPN HYDROLASE

Activity $\mathrm{NAD}(+)+\mathrm{H}(2) \mathrm{O})=\mathrm{NIC}\left(\mathrm{O}^{\prime}\right.$ TINAMIDE + AI)P-RIBOSE.

P'ep???? cds???" Pos"??? Loc????

Reaction: NAD_NAD_3

$$
\text { ATP } 271.23 \quad \mathrm{ADP}
$$

2.7.1.23: $\mathrm{NAI}^{+}$Kinass

\section{DESCRIPTIONS OF THE ENZYME}

desc NAD(t) KINASE

Alternate name DPN KINASE

Activity $\mathrm{ATP}+\mathrm{NAD}(+)=\mathrm{ADP}+\mathrm{NADP}(+)$. 
Pep???? cds???? Pos???? Loc???

Reaction: Niacin_NAD_1

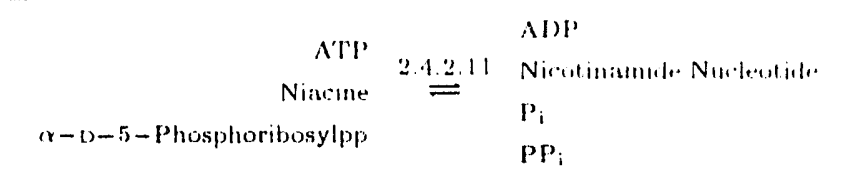

2.4.2.11: Nicotinate Phosphoribosyltransferase

P18133 P22253

DESCRIPTIONS OF THE ENZYME

desc NICOTINATE PHOSPHORIBOSYLTRANSFERASE

Activity NICOOTINATE D-RIBONUICLEOTIDE + P'YROPHOSPHATE

= NICOTINATE + 5-PHOSPHO-ALPHA-D-RIBOSE I-DIPHOSFHATE.

P18133 pncB 21.19995834 from 997036 complement

DESCRIPTIONS OF THE PEPTIDE

desc NICOTINATE PHOSPHORIBOSYLTRANSFERASE (EC: 2.4.2.11)

E.coli gene PNC:B

DESCRIPTIONS OF THE CINS

desc Nirotinate phosphoribosyltransferase:

Reaction: Niacinamide_NAD_2

$$
\begin{array}{r}
\text { Niacinamide } \\
\mathrm{H}_{2} \mathrm{O}
\end{array} \stackrel{3.5 .1 .19}{=} \begin{aligned}
& \text { Ammonia } \\
& \text { Niacine }
\end{aligned}
$$

3.5.1.19: Nicolinamidase

DESCRIPTIONS OF THE ENZYME

desc NICOTINAMIDASE

Activity NICO'TINAMIDE $+\mathrm{H}(2) \mathrm{O}=$ NICOTINA'TE + NII(:3). 
Pep??? pucA 38.79 Loc????

\section{DESCRIPTIONS OF THE CDS}

desc nicotinamide deamirlase

Reaction: Nicotinamide_Nucleotide_NAD_2

$$
\begin{aligned}
& \text { Nicotinamide Nucleotide } \mathbf{3 . 5 . 1 . 0 0} \text { Ammonia } \\
& \mathrm{H}_{2} \mathrm{O} \rightleftharpoons \text { Nicotinate Nucleotide }
\end{aligned}
$$

\section{5 .1 .00}

Pep???? cds??? Pos???? Loc????

Reaction: Nicotinamide_Ribose_NAD_1

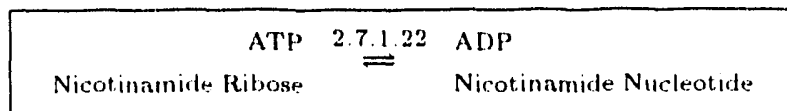

2.7.1.22: Ribosylnicolinamide Kinase

\section{DESCRIPTIONS OF THE ENZYME}

desc RIBOSYLNICOTINAMIDE KINASE

Activity ATP + N-RIBOSYINICOTINAMIDE $=$ ADP + NICO'TINAMIDE RIBONICLEOTIDE.

Pep??? cels??? Pos??? Loc???

\section{Reaction: Ubiquinone 1}

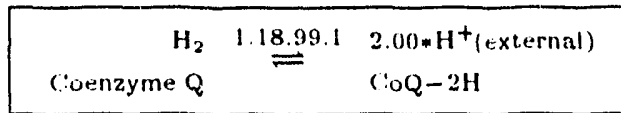

\begin{tabular}{|c|c|c|c|c|}
\hline & 9 & & & \\
\hline & & & & \\
\hline & & & & \\
\hline & 7 & & & \\
\hline & & & & \\
\hline 1 & 13065 & $P 13066$ & P13063 & P1306 \\
\hline
\end{tabular}

1 18.99.1: Hydrogenase 
DESCRIPTIONS OF THE ENZYME

desc HYDROCIENASE

Alternate name HYI)RO(IENLYASE

Activity 2 REDU(IEI) FERREDO)XIN $+2 \mathrm{H}(+)=2$ ()XII)IZEI) FERRED)()XIN + H(2).

P19927 hyaB Pos???? 1041049 to 1042842

Descriptions of THE Pep'TIDE

desc HYDROGENASE-1 LARGE CHAIN (EC: 1.18.99.1) (NIFE HYDRO(IENASE)

E.coli gene HYAB

Plo928 hyad Pos???? 10339934 to 1041052

Descriptions of THE PEPTIDE

desc HYDRO(IENASE-1 SMALL (HAIN PRE(:URSOR (E) :1.18.99.1) (NIFE HYDRO(iENASE)

E.coli gene HYAA 


\section{Index}

\section{Enzymes}

1.1.1.100 (3-0xoacyl-[Acyl-('arrier P'rotein] Reductase), 108

1.1.1.205 (IMP' Dehydrogentasion), 44, 130

1.1.1.23 (Histidinol Dehydrogenase), 73

1.1.1.25 (Shikimate Dehydrogenase), 12

1.1.1.3 (Homoserine Dehydrogenase), 76

1.1.1.35 (3-Hydroxyacyl-('oA Dehydrogenase), 41

1.1.1.37 (Malate Dehydrogenase), 148

1.1.1.42 (Isocitrate Dehydrogenase (NADP+ )), 143

1.1.1.44 (6-Phosphogluconate Dehydrogenase (Decarboxylating)), 111

1.1.1.49 (Cilucose-6-P'hosphate Dehydrogenase), 110

1.1.1.86 (Ketol-Acid Reductoisomerase), 89, 164, 169

1.1.1.95 (Phosphoglycerate Dehydrogenase), 139

1.18.99.1 (Hydrogenase), 180

1.2.1.11 (Aspartate-Semialdehyde Dehydrogenase), 75

1.2.1.12 (Gilyceraldehyde 3-Phosphate Dehydrogenase), 50, 64

1.2.1.38 (N-Acetyl- $\gamma$-Cilutamyl-Phosphate Reductase), 5)

1.2.1.41 ((ilutamate-5-Semialdehyde Dehydrogenase), 118

1.2.4.1 (Pyruvate Dehydrogenase (Lipoamide)), 141

1.2.4.2 (()xoglutarate Dehydrogenase (Lipoamide)), 144

1.3.1.10 (Enoyl-[Acyl-('arrier Protein] Reductase (NADPH, B-Specific)), 109

1.3.1.12 (Prephenate Dehydrogenase), 160

1.3.1.26 (Dihydrodipicolinate Reductase), 93

1.3.3.1 (Dihydroorotate Oxidase), 135

1.3.99.1 (Sucrinate Dehydrogenase), 31, 145

1.4.1.13 (Gilutamate Synthase (NADPH)), 58

1.4.1.4 (Gilutamate Dehydrogenase $\left.\left(N A D P^{+}\right)\right), 57$

1.5.1.2 (Pyrroline-5-Carboxylate Reductase), 119

1.5.1.5 (Methylenetetrahydrofolate Dehydrogenase $\left(N A D P^{+}\right)$), 150

1.6.1.1 (NAD $\left(P^{P}\right)^{+}$Transhydrogenase (B-Specific)), 176

1.6.99.3 (NADH Dehydrogenase), 24 
2,3,4,5-Tetrahydropyridine-2-('arboxylate N-Succinyltransferase (E.(.. 2..3.1.117), 93

2.1.1.13 (5-Methyltetrahydrofolate-Homocysteine Methyltransferase), 99

2.1.1.14 (5-Methyltetralyvdropteroyltriglutamate-Homocysteine Methyltransferase), 102

2.1.1.2((inandinoaretate Methyltransferase), 1!)

2.1.2.1 (Gilycine Hydroxymethyll ransferase), 173

2.1.2.2 (Phosphoribosylglycinamide Formyltransferase), 79, 121

2.1.2.3 (Phosphoribosylaminoimidazolecarboxamide Formyltransferase), 84, 122

2.1.3.2 (Aspartate (arbamoyltransferase), 133

2.1.3.3 (Ornithine (arbamoyltransferase), 7

2.1.4.1 (Glycine Amidinotransferase), 19

2.2.1.1 (Transketolase), 113

2.2.1.2 (Transalḍolase), 114

2.3.1.1 (Amino-Acid Acetyltransferase), 4

2.3.1.117 (2,3,4,5-Tetrahydropyridine-2-Carboxylate $N$-Succinylt ransferase), 93

2.3.1.16 (Acetyl-Coa Acyltransferase), 42

2.3.1.30 (Serine Acetyltransferase), 20

2.3.1.38 ([Acyl-('arrier P'rotein] Acetyltransferase), 106

2.3.1.39 ([Acyl-Carrier Protein] Malonyltransferase), 107

2.3.1.41 (3-Oxoacyl-[Acyl-('arrier Protein] Synthase), 107

2.3.1.46 (Homoserine suncinyltransferase), 97, 100

2.4.1.21 (starch (Bacterial (ilycogen) synthase), 56

2.4.2.10 (Orotate Phosphoribosyltransferase), 135)

2.4.2.11 (Nirotinate Phosphoribosyltransferase), 179

2.4.2.12 (Nicotinamide Phosphoribosyltransferase), 1033

2.4.2.14 (Amidophosphoribosyltransferase), 78, 12:3

2.4.2.17 (ATP Phosphoribosyltransferase), 68

2.4.2.18 (Anthranilate Phosphoribosyltransferase), 15.5

2.4.2.19 (Nicotinate-Nucleotide Pyrophosphorylase (Carboxylating)), 104

2.5.1.19 (3-Phosphoshikimate 1-Carboxyvinyltransferase), 14

2.5.1.6 (Methionine Adenosyltransferase), 22

2.6.1.1 (Aspartate Aminotransferase), 176

2.6.1.11 (Acetylornithine Aminotransferase), 6 
2.6.1.17 (Siucingldiaminopimelate Aminotransferase), 94

2.6.1.2 (Alanine Aminotransferase), 172

2.6.1.42 (Brandhed-Chain Amino Acid Aminotransferase), 90, 165)

2.6.1.5 (Tyrosine Aminotransferase), 116, 160

2.(j.1.52 (Phosphoserine Aminotransferase), 139)

2.6.1.6ifi (Valine-Pyruvate Aminotransferaste), 170

2.6.1.9 (Histidinol-Phosphate Aminotransferase), 71

2.7.1.11 (6-Phosphofructokinase), 61

2.7.1.2 (Cilucokinase), 60,110

2.7 .1 .22 (Ribosylnicotinamide Kinase), 180

2.7.1.2:3 (NAD) Kinase), 178

2.7 .1 .24 (Dephospho-('od Kinase), I8

2.7.1.26 (Ribollavin Kinase), 39)

2.7.1.333 (Pantothenate Kinase), 16

2.7.1.39) (Humoserine Kinase), 152

2.7.1.40 (Pyruvate Kinase), 67

2.7.1.7! (Shikimate Kinase), 13

2.7.2.11 (Glutamate 5-Kinase), 118

2.7.2.3 (Phosphuglycerate Kinase), 49, 65

2.7.2.4 (Aspartate Kinase), 74

2.7.2.8 (Acetylglutamate Kinase), 5

2.7.3.2 (('reatine Kinase), $17 \%$

2.7.4.3 (Adenylate Kinase), 2, 128

2.7.4.6 (Nucleoside-Diphosphate Kinase), 3, 46, 128, 130,137

2.7.4.8 (Guanylate hinase), 45, 131

2.7.5.3 (Transferred Entry: 5.4.2.1), 66

2.7.6.1 (Ribose-Phosphate Pyrophosphokinase), 77, 123

2.7.7.1 (Nicotinamide-Nucleotide Adenylyltransferase), 103

2.7.7.18 (Nicotinate-Nucleotide Adenylyltransferase), 104

2.7.7.2 (FMN Adenylyltransferase), 39

2.7.7.27 (Cilucose-1-Phosphate Adenylyltransferase), 55

2.7.7.3 (Pantetheine-Phosphate Adenylyltransferase), 17

3-Dehydroquinate Dehydratase (E.C. 4.2.1.10), 12 
3-Dehydroquinate Synthase (E.(:. 4.6.1.3), 11

3-Hydroxyacyl-('oA Dehydrogenase (E.c. 1.1.1.3.5), $4 \mathrm{I}$

3-Oxoacyl-[Acyl-('arrier Protein] Reductase (E.C. 1.1.1.100), 108

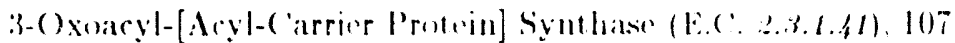

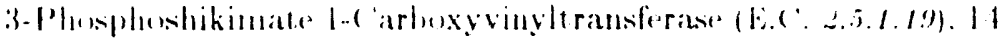

3.1.1.31 (6-Phosplengluconolatonase), 111

$3.1 .2 .00,177$

3.1.3.11 (Fructose-Bisphosphatase), 533

3.1.3.15 (Histidino!-Phosphatase), 72

3.1.3.3 (Phosphoserine Phosphatase), 140

$3.2 .2 .5(\text { NAD })^{+}$Nucleosidase), 178

$3.5 .1 .00,180$

3.5.1.16 (Aretylornithine Deacetylase), 7

3.5.1.18 (Succinyldiaminopimelate Desuccinylase), 94

3.5.1.19 (Nicotinamidase), 179

3.5.2.10 (Creatininase), 172

3.5.2.3 (Dihydroorotase), 134

3.5.4.10 (IMP Cyclohydrolase), 84, 124

3.5.4.19 (Phosphoribosyl-AMP Cyclohydrolase), 69

3.5.4.9 (Methenyltetrahydrofolate Cyclohydrolase), 150

3.6.1.22 (NAD) Pyrophosphatase), 177

3.6.1.31 (Phosphoribosyl-ATP Pyrophosphatase), 69

3.6.1.34 ( $\mathrm{H}^{+}$-Transporting ATP' Synthase), 26,34

4.1.1.20 (Diaminopimelate Decarboxylase), 95

4.1.1.21 (Phosphoribosylaminoimidazole (arboxylase), 81, 124

4.1.1.23 (Orotidine-5'-Phosphate Decarboxylase), 136

4.1.1.36 (Phosphopantothenoylcysteine Decarboxylase), 17

4.1.1.48 (Indole-3-Gilycerol Phosphate Synthase), 157

4.1.1.49 (P'hosphoenolpyruvate Carboxykinase (ATP)), 47

4.1.2.13 (Fructose-Bisphosphate Aldolase), 51, 62

4.1.2.15 (Phospho-2-Dehydro-3-Deoxyheptonate Aldolase), 10

4.1.3.18 (Acetolactate Synthase), 87, 162, 167

4.1.3.27 (Anthranilate Synthase), 154 
4.1.3.7 ((itrate (Si)-Synthase), 142

4.2.1.10 (3-Dehydroquinate Dehydratase), 12

4.2.1.11 (Enolase), 48, 66)

4.2.1.16 (Threonine Dehydratase), 86

1.2.1.17 (Enoyl-( od Hydratiare) 41

4.2.1.19 (Imidazoleglycerol-l'hosphate Dehyclratiase), 71

4.2.1.2 (Fumarate Dehydratase), 147

4.2.1.20 (Tryptophan Synthase), 157

4.2.1.22 (Cystathionine B-synthase), 23

4.2.1.3 (Aconitate Hydratase), 142

4.2.1.51 (Prephenate Dehydratase), 116

4.2.1.52 (Dihydrodipicolinate iynthise), !)

4.2.1.58 (Crotonoyl-[Acyl-(arrier Protein] Hydratase), 108

4.2.1.9 (Dihydroxy-Acid Dehydratase), 90, 165), 170

4.2.99.2 (Threonine Synthase), 152

4.2.99.8 (O-Acetylserine (Thiol)-Lyase), 20

4.2.99.9 (O-Succinylhomoserine (Thiol)-Lyase), 97, 100

4.3.2.1 (Argininosuccinate Lyase), 9

4.3.2.2 (Adenylosuccinate Lyase), 1, 83, 125), 129

4.4.1.1 (Cystathionine $\gamma$-Lyase), 23

4.4.1.8 (Cystathionine- $\beta-L y a s e), 98,101$

4.6.1.3) (3-Dehydroquinate Synthase), 11

4.6.1.4 (Chorismate Synthase), 14

5)-Methyltetrahydrofolate-Homocysteine Methyltransferase (E.(: 2..1.1.1.3), 99)

5-Methyltetrahydroptroyltriglutamate-Homocysteine Methyltransferast: (E.c:.

2.1.1.1k), 102

5.1.1.7 (Diaminopimelate Epimerase), 95

5.1.3.1 (Ribulose-Phosphate 3-Epimerase), 112

5.3.1.1 (Triosephosphate Isomerase), 52, 63

5.3.1.16 (Imidazole Carboxamide Isomerase), 70

5.3.1.24 ( $N$-(.5'-Phosphoribosyl) Anthranilate Isomerase), 156

5.3.1.6 (Ribose 5-Phosphate Epimerase), 112

5.3.1.9 ((ilucose-6-Phosphate lsomerase), 54, 60 
5.4.2.1 (Phosphoglycerate Mutase), 48

5.4 .2 .2 (Phosphoglucomutase), 55)

5.4 .99 .5 (('horismate Mutase), 115, 159

(i-Phusphofructokinass (E.c. :..7.1.11),61

(j)-Phosphogluconate Dehydrogenase (Decarboxylating) (E.C: 1.1.1.1.4), 111

6-Phosphogluconolactonase (E.(: 3.1.1.31), 111

6.2.1.3 (Long-Chain-Fatty-Arid-CoA Ligase), 40

6.2.1.5 (Succinate-( OA Ligase (ADP-Forming)), 144

6.3.1.1 (Aspartate-Ammonia Ligase), 175

6.3.1.2 ((ilutamate-Ammonia Ligase), .58

6.3.1.4 (Aspartate-Ammonia Ligase (AD)P-Forming)), 175

6.3.2.5 (Phosphopantothenate-(ysteine Ligase). 16

6.3.2.6 (Phosphoribosylaminoimidazole-Succinocarboxamide Syuthase), 62, 125)

6.3.3.1 (Phosphoribosylformylglycinamidine (yclo-Ligase), 81,126

6.3.4.13 (Phosphoribosylamine-Cilycine Ligase), 7\$, 127

6.3.4.2 (CTP Synthase), 137

6.3.4.3 (Formate-Tetrahydrofolate Ligase), 151

6.3.4.4 (Adenylosurrinate Synthase), 1, 129

6.3.4.5 (Argininosucrinate Synthase), 8

6.3.5.1 (NAD) Synthase (Cilutamine-Hydrolysing)), 105

6.3.5.2 ((iMP' Synthase (Cilutamine-Hydrolysing)), 45, 1:31

6.3.5.3 (Phosphoribosylformylglycinamidine Synthase), 80,127

6.3.5.4 (Asparagine Synthase ((ilutamine-Hydrolysing)), 174

6.3.5.5) (Carbamoyl-Phosphate Synthase (Cilutamine-Hydrolysing)), 1.32

6.4.1.2 (Acetyl-('oA ('arboxylase), 106

Acetolactate Synthase (E.(: 4.1.3.18), 87, 162, 167

Acetyl-('oa Acyltransferase (E.(. 2.3.1.16), 42

Acetyl-('oA Carboxylase (E.(: 6.4.1.2) 106

Acetylglutamate Kinase (E.(: 2.7.7.2.8), 5

Acetylornithine Aminotransferase (E.(:. 2.6.1.11), 6

Acetylornithine Deacetylase (E.(:. 3.5.1.16), 7

Aconitate Hydratase (E.(: \&.2.1.3), 142 
Aryl-('oA Dehydrogenase, 40

Adenylate Kinase (E.C. 2.7.4.3), 2, 128

Adenylosuccinate Lyases (E.( $\therefore$ 4.3.2.2), 1, 8.3, 125, 129)

Adenylosucrinate Synthase (E.( 6.3 .4 .4$), 1,129$

Alanine Aminotransferase (E.(: 2.6.1.2.2) 172

Amiclocycliase, 71

Amidophosphoribosyltransferase (E.C. 2.4.2.14), 78, 123

Amino-Acid Acetyltransferase (E.(:. 2.3.1.1), 4

Anthranilate Phosphoribosyltransferase (E.(. ..4.2.18), 15.5

Anthranilate Synthase (E.(: 4.1.3.27), 154

Argininosuccinate Lyase (E.( . 4.3.2.1), 9

Argininosucrinate Synthase (E.(: . 6.3.4.5), 8

Asparagine Synthase ((ilutamine-Hydrolysing) (E.(. 6.3.5.4), 174

Aspartate Aminotransferase (E.(:. 2.6.1.1), 176

Aspartate ('arbamoyltransferase (E.(.....1.3.2), 1333

Aspartate Kinase (E.C. 2.7.2.4), 74

Aspartate-Ammonia Ligase (ADP-Forming) (E.( $\therefore$ 6.3.1.4), 175)

Aspartate-Ammonia Ligase (E.(: 6.3.1.1), 175)

Aspartate-Semialdehyde Dehydrogenase (E.(:. 1.2.1.11), 75

ATP Phosphoribosyltransferase (E.(‥2.4.2.17), 68

Branched-( hain Amino Acid Aminotransferase (E.(:. 2.6.1.409), 90, 165

('arbamoyl-Phosphate Synthase (Cilutamine-Hydrolysing) (E.(: 6.3.5.5), 132

('horismate Mutase (E.( $\therefore$. 5.4.99.5), 115, 159

('horismate Synthase (E.(:. 4.6.1.4), 14

(.itrate (Si)-Synthase (E.C: 4.1.3.7), 142

(reatine Kinase (E.C. 2. 2.3.3.2), 172

C'reatininase (E.(. 3.5.9.10), 172

('rotonoyl-[Acyl-('arrier Protein] Hydratase (E.(. 4.2.1.58), 108

(.TP Synthase (E.(: 6.3.4.2), 137

(ystathionine $\beta$-Synthase (E.C. 4.2.1.20), 23

(ystathionine $\gamma$-Lyase (E.(: . 4.4.1.1), 23

('ystathionine- $\beta$-Lyase (E.C. 1.1.1.8), 98, 101 
Dephospho-('oA Kinase (E.(: .2.7.1.24), 18

Diaminopimelate Decarboxylase (E.(. 4.1.1.20), 95)

Diaminopimelate Epimerase (E.c. 5.1.1.7), 95

Dihydrodipicolinate Reductasese (E.c. 1.3.1.96), 93

Dihydrodipicolinate Syuthase (E.( $\therefore$, 1.2.1.52), 92

Dihydroorotiase (E.(. .3.5.8.3), 134

Dihydroorotate Oxidase (E.(: 1.3.3.1), 135)

Dihydroxy-Acid Dehydratiase (E.C. 4.2.1.9), 90, 165, 170

Enolase (E.(:. 4.2.1.11), 48, 66

Enoyl-('oA Hydratase (E.(: 1.2.2.17), 41

Enoyl-[Acyl-('arrier Protein] Reductase (NADPH, B-Specific) (E.(: 1.3.1.10), 109

FMN Adeuylyltransferase (E.(…7.7.2), 39

Formate-Tetrahydrofolate Ligase (E.C. 6.3.4.3), 151

Fructose-Bisphosphatase (E.(. 3.1.3.11), 5.3

Fructose-Bisphosphate Aldolase (E.C. 4.1.2.13), 51, 62

Fumarate Dehydratase (E.C. 4.2.1.2), 147

Clucokinase (E.C. …7.1.2), 60, 110

(ilucose-1-Phosphate Adenylylt,ransferase (E.(: 2.7.7.27), 5.5)

(ilucose-6-Phosphate Dehydrogenase (E.C. 1.1.1.49), 110

(ilucose-(i)-Phosphate lsomerase (E.(. 5.3.1.9), 54, 60

(ilutamate 5-Kinase (E.C. . . 7.2.11), 118

(ilutamate Dehydrogenase (NADP+) (E.(.. 1.4.1.4), 57

(ilutamate Synthase (NADPH) (E.(: 1.4.1.1.3), 58

(ilutamate-Ammonia Ligase (E.C. B.3.1.2), 58

(ilutamate-5)-Semialdehyde Dehydrogenase (E.(.. 1.2.1.41), 118

(ilyceraldehyde 3-Phosphate Dehydrogenase (E.C. 1.2.1.12), 50, 64

(ilycine Amidinotransferase (E.(.. 2.1.4.1), 19

glycine cleavage enzymes, 173

(ilycine Hydroxymethyltransferase (E.(‥2.1.2.1), 173

(iMP Synthase (Cilutamine-Hydrolysing) (E.C. 6.3.5.2), 45, 131

(inanidinoacetate Methyltransferase (E.(‥2.1.1.2), 19) 
(iuanylate Kinase (E.(: 9.7.4.8), 45, 1:31

Histidinol Dehydrogenaste (E.(: 1.1.1.2.3), 73

Histidinol-Phosphatiase (E.(‥3.1.3.1.5), 72

Histiclinol-Phosphate Aminotransferase (E.(…6.1.9), 71

Homoserine Dehydrogenase (E.(:. 1.1.1.3), 76

Homoserine Kinase (E.(. . 9.7.1.39), 152

Homoserine Succinyltransferase (E.C. 2..3.1.46), 97, 100

Hydrogenase (E.(. 1.18.99.1), 180

$\mathrm{H}^{+}$-Transporting ATP Synthase (E.C. 3.6.1.34), 26, 34

Imidazole ('arboxamide Isomerase (E.(:. 5.3.1.16), 70

Imidazoleglycerol-Phosphate Dehydratase (E.(:.4.2.1.19), 71

IMP ('yclohydrolisse (E.) 3.5.4.10), 84, 124

IMP Dehydrogenase (E.(:. 1.1.1.20.5), 44, 130

Indole-3-(ilycerol Phosphate Synthase (E.(. 4.1.1.48), 157

Isocitrate Dehydrogenase (NADP ${ }^{+}$) (E.C. 1.1.1.42), 143

Ketol-Acid Reductoisomerase (E.(. 1.1.1.86), 89, 164, 169

Long-('hain-Fatty-Acid-(.oA Ligase (E.C. 6.2.1.3), 40

Malate Dehydrogenase (E.c: 1.1.1.37), 148

Methenyltetrahydrofolate (:yclohydrolase (E.(: 3.5.4.9), 150)

Methionine Adenosyltransferase (E.(:. 2.5.1.6), 22

Methylenetetrahydrofolate Dehydrogenase (NADP+) (E.(. 1.5.1.5), 150

$\mathrm{N}-(5$-Phosphoribosyl)Anthranilate Isomerase (E.(: 5.3.1.24), 156

$\mathrm{N}$-Acetyl- $\gamma$-(ilutarnyl-Phosphate Reductase (E.C. 1.2.1.38), 5

NAD $(P)+$ Transhydrogenase (B-Specific) (E.(. 1.6.1.1), 176

NADH Dehydrogenase (E.(: 1.6.99.3), 24

$\mathrm{NAD}{ }^{+}$Kinase (E.C. 2.7.1.23), 178

$\mathrm{NAD}^{+}$Nucleosidase (E.C. 3:2.2.5), 178

NAD + Pyrophosphatase (E.(: 3.6.1.22), 177

$\mathrm{NAD}^{+}$Synthase ((ilutamine-Hydrolysing) (E.C. 6.3.5.1), 105)

Nicotinamidase (E.(: 3.5.1.19), 179 
Nicotinamide Phosphoribosyltransferase (E.(‥2.4.2.12), 103

Nicotinamide-Nucleotide Adenylyltransferase (E.(.. 2.7.7.1), 103

Nicotinat.e Phosphoribosyltransferase (E.C. 2.4.2.11), 179

Nicotinate-Nucteotide Adenylylt.ransferase (E.(…7.7.18), 104

Nicotinate-Nucleotide Pyrophosphorylase (('arboxylating) (E.c. :.4.2.19), 104

Nucleoside-Diphosphate Kinase (E.(․ .2.7.4.6), 3, 46, 128, 130, i37

O-Acetylserine (Thiol)-Lyase (E.(․ 4.2.99.8), 20

O-Succinylhomoserine (Thiol)-Lyase (E.(. 4.2.99.9), 97, 100

Ornithine (arbamoyltransferase (E.C. . . 1.3.3), 7

Orotate Phosphoribosyltransferase (E.C. 2.4.2.10), 135)

()rotidine-5)-Phosphate Decarboxylase (E.(..4.1.1.2.3), 136

Oxoglutarate Dehydrogenase (Lipoanide) (E.(. 1.9.4.2), 144

Pantetheine-Phosphate Adenylyltransferase (E.(. . . 7.7.3), 17

Pantothenate Kinase (E.(.. 9.7.1.33), 16

Phospho-2-Dehydro-3-Deoxyheptonate Aldolase (E.C. 4.1.2.15), 10

Phosphoenolpyruvate ('arboxykinase (ATP) (E.C. 4.1.1.49), 47

Phosphoglucomutase (E.(., 5.4.2.2.2), 5.5

Phosphoglycerate Dehydrogenase (E.C. 1.1.1.95), 139

Phosphoglycerate Kinase (E.(:. 2.7.2.3), 49, 65)

Phosphoglycerate Mutasse (E.(: 5.4.2.1), 48

Phosphopantothenate-(ysteine Ligase (E.C. 6.3.2.5), 16

Phosphopantothenoylcysteine Decarboxylase (E.(: 4.1.1.36), 17

Phosphoribosyl-A MP ('yclohydrolase (E.C. 3.5.4.19), 69

Phosphoribosyl-ATP Pyrophosphatase (E.C. 3.6.1.31), 69

Phosphoribosylamine-(ilycine Ligase (E.(: 6.3.4.13), 78, 127

Phosphoribosylaminoimidazole (Carboxylase (E.C. 4.1.1.21), 81, 124

Phosphoribosylaminoimidazole-Succinocarboxamide Synthase (E.(: 6.3.2.6), 82, 125

Phosphoribosylaminoimidazolecarboxamide Formyltransferase (E.C.. 2. 1.2.3), 84, 122

Phosphoribosylformylglycinamidine (yyclo-Ligase (E.c. 6.3.3.1), 81, 126

Phosphoribosylformylglycinamidine Synthase (E.C. 6.3.5.3), 80, 127 
Phosphorihosylglycinanide Formyltransferase (E.(‥2.1.20), 79, 121

Phosphoserine Aminotransferase (E.(․ 2.6.1.5.2), 139)

Phosphoserine Phosphatiase (E.(‥3.1.3.3), 140)

Prephenate Dehydratase (li.c - 1.2.2.51), 116

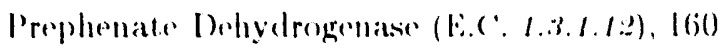

Pyrroline-5)-('arboxylate Rerductiase (K..(: 1.5.1.2), 119)

Pyruvate carboxylase, 17

Pyruvate Dehyctrogenase (Lipoamide) (E.C. 1.2.4.1), 141

Pyruvate Kinase (E.(: : 2.7 .40 ), 67

Rihollavin Kinase (E.(: 2.7 .1 .26$), 39$

Rihose 5)-Phosphate Epimerase (F.(: 5.3.1.65), 112

Rihose-Phosphate Pyrophosphokinase (E.c. : 2.7.6.t), 77, 12:3

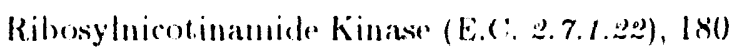

Ribulose-Phosphate 3-Epimerase (E.(: 5.1.3.1), 112

Serine Acetyltransferase (E.( .2 .3 .1 .30$), 20$

Shikimate Dehydrogenase (E.c. 1.1.1.2.5), 12

Shikimate Kinase (E.(…7.1.7I), 13

Starch (Bacterial (ilycogen) Synthase (E.(‥2.4.1.21), 56

Succinate Dehydrogenase (E.( : 1.3.99.1), 31, 145)

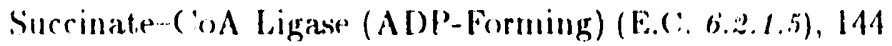

Sucringldiaminopimelate Aminotransferase (E.(2. 2.6.1.17). 94

Succinyldiaminopimelale Desuccinylase (E.c. 3.5.1.18), 94

Threonine Dehydratase (E.(‥4.2.1.16), 86

Threonine Synthase (E.c. 4.2.99.2), 152

Transaldolase (E.(:. 2..2.1.2.2), 114

Transferred Entry: 5.4.2.1 (E.(:.9.7.5.3), 66

Transketolase (E.(…2...1.1), 113

Triosephosphate Isomerisese (E.1., 5.3.1.1), 52, 63)

Tryptophan Synthase (E.(: 4.2.1.20), 157

Tyrosine Aminotransferase (E.(:. 2.6.1.5), 116, 160

Uriclylate kinaste, 137 
Valine-Pyruvate Aminotransferase (E.(‥2.6.1.66), 170

[Acyl-('arrier Protein] Acetyltransferase (E.(‥2.3.1.38), 106

[Acyl-('arrier P'rotein] Malonyltransferase (E.(:. 2..3.1.39), 107

\section{Genes}

ace $E, 141$

$a d k, 2,128$

$\arg A, 4$

$\arg B, 5$

$\arg (: 6$

$\arg l), 6$

$\arg E, 7$

$\arg F, 8$

$\arg (i, 9$

arg $H, 9$

$\operatorname{argl}, 8$

aro $A, 14$

aro $B, 12$

aro $(, 15$

arol), 12

aro $E, 13$

aro $F, 11$

aroli, 10

aroH, 11

aro $L, 13$

asd, 75

asn A, 175

asn B, 174

aspe:, 176

$\operatorname{atp} A, 28,36$

atp $B, 29,37$

atp $(;, 29,37$ 


$$
\begin{aligned}
& \text { atp D, 28, 36 } \\
& \text { alp } E, 29,37 \\
& \text { alp } F, 30,35 \\
& \text { alp }(i, 29,37 \\
& \text { alpH, } 28,36 \\
& \text { antA, } 171 \\
& \operatorname{car} A, 132 \\
& \operatorname{car} B, 13: 3 \\
& \text { cys } E, 20 \\
& \text { cysh, } 21 \\
& \text { rys } M, 21 \\
& \operatorname{dap} A, 92 \\
& \text { dap } B, 93 \\
& \text { dap }[), 94 \\
& \operatorname{dap} F, 95 \\
& \text { eno, } 48,66 \\
& f a b B, 108 \\
& \text { fabD), } 107 \\
& \text { fabE, } 107 \\
& \text { fad } A, 4: \\
& \text { fad } B, 41,42 \\
& \text { fadD, } 40 \\
& f h a, 5 l, 63 \\
& f b p, 53 \\
& \text { frd } A, 31,146 \\
& \text { frd } B, 32,146 \\
& \text { fum } A, 147 \\
& \text { fum } B, 148 \\
& \text { fum C, } 148 \\
& \text { gap } A, 50,64
\end{aligned}
$$




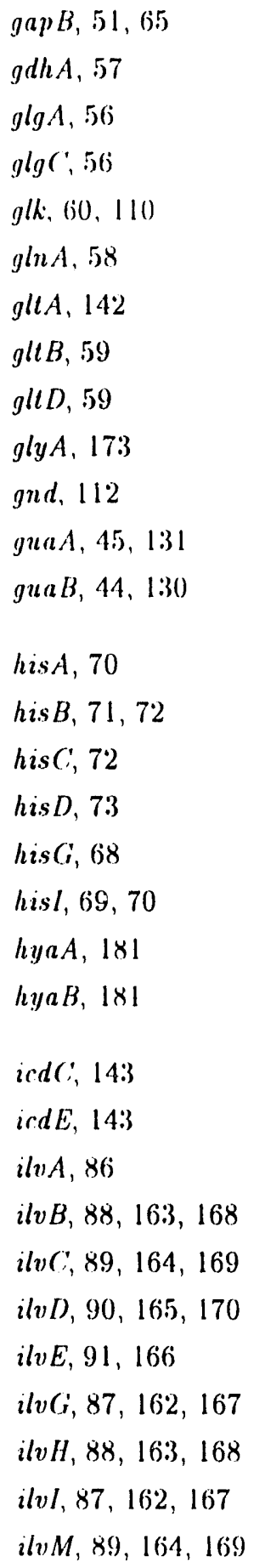


$i \operatorname{lvN}, 88,163,168$

lys A, 96

lys (', 75)

milh, 149

metA, 97, 100

met $B, 98,101$

metc, $23,98,101$

mete, 102

meth, 99

meth, 22

melL, 74,76

$n d h, 24$

$p c k A, 48$

$p f k A, 62$

$p f k B, 62$

$p g i, 54,61$

$p g k, 49,65$

$p g l, 111$

phe $A, 115,116,159$

pne $A, 180$

pnc $B, 179$

pntA, 177

$p n t B, 177$

proA, 119

proB, 118

proc: 120

prs, 77, 123

purA, 1, 129

purB, 2, 83, 125, 129

purf:, 83,126

purl), 79, 127 


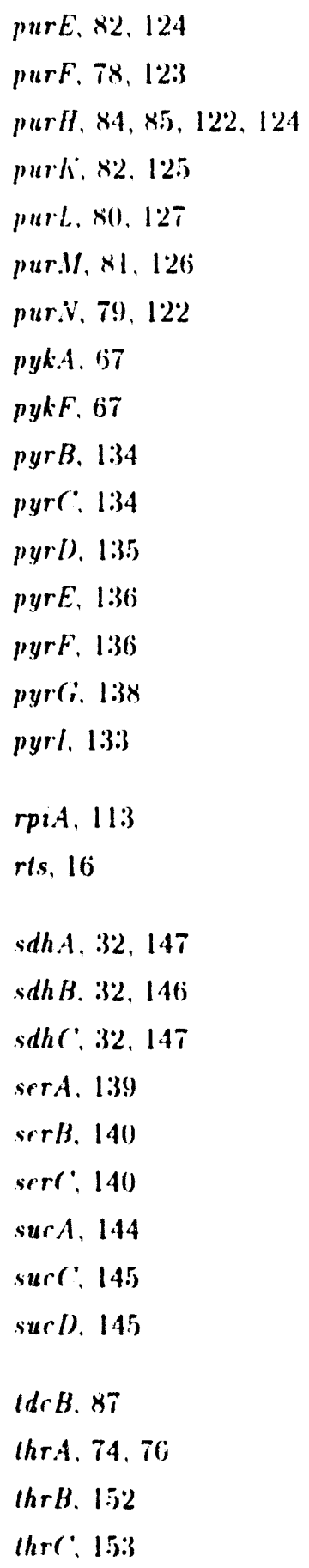


tht, 113, 114

tpiA, 52, 53, 633

1rp.A, 15s

trpB. 15s

Irpe: 1566, 157

$\operatorname{lrp}(), 155$

$\operatorname{trp} E, 154$

tyrA, 115, 159, 160

$\operatorname{tyr} B, 117,161$

$: w f .111$

\section{Peptides}

P00346, 14 א

P00348, 41

P00349, 111

P00350, 111, 112

P00353, 75

P00354, 50, 64

P00355, 50, 64

P00356, 50, 64

P00357, 50, 64

P00358, 50, 64

P00359, 50, 64

P00360, 50, 64

P00361、 sid. 64

P00362, 50.64

P00363, 31, 145, 146

P00364, 31, 32, 145, 146

P00369, 57

P00:370, 57

P00373, 119, 120

P(0):393, 24 
P00477, 173

P.9478, 133

Puns, 133, 134

ลง 010480,7

P00481, T

P00495, 135, 136

P00496. 78, 123

P00497, 78, 12:3

P00498, 68

P00499, 68

P00500, 155)

P00503, 176

P00504, 176

P00505, 176

P00506, 176

P00507, 176

P00508, 176

P00509, 176

P00510, 90, 91, 165, 166

P00511, 61

P00512, 61

P00547, 152

P00548, 67

P00549, 67

P00558, 49, 65

P00559, 49, 65

P00560, 49, 65

P00561, 74, 76

P00562, 74, 76

P00568, 2, 128

P00569, 2, 128

P00570, 2, 128

P00571, 2, 128 
$P 00584,55,56$

P00636, 5.3

P00637, 53:3

P00815, 69, 73

P00822, 27, 28, 35, 36

P00823, 27, 28, 35, 36

P00824, 27, 28, 35, 36

P00825, 27, 28, 35, 36

P00826, 27, 28, 35, 36

P00827, 27, 28, 35, 36

P00828, 27, 28, 35, 36

P00829, 27, 35

P00830, 27, 35

P008:31, 27, 28, 35), 36

P008:32, 27, 29, 35, 37

P00833, 27, 35

P00834, 27, 35

P00835, 27, 35

P00836, 27, 35

P008:37, 27, 29, 35, 37

P00839, 27, 35)

P00840, 27, 35

P00841, 27, 35

P00842, 27, 35)

P00843, 27, 35

P00844, 27, 29, 35, 37

P00845, 27, 35

P00846, 27, 35)

P00847, 27, 355

P00848, 27, 35)

P00849, 27, 35

P00850, 27, 35)

P00851, 27, 35 
P00852, 2T, 35)

P00853, 27, 35

P00854, 27, 35

P00855, 27, 29, 35, 37

P00856, 27, 35)

P00857, 27, 35

P00858, 27, 35)

P00859, 27, 30, 35., 38

P00861, 95, 96

P00883, 51, 62

P00884, 51, 62

P00886, 10

P00887, 10, 11

P00888, 10, 11

P00889, 142

P00890, 142

P00891, 142

P00892, 87, 162, 167

P00893, 87, 162, 167

P00894, 87, 88, 162, 1633,167, 168

P00895, 154

P00896, 154

P00897, 154

P00898, 154

P00899, 154

P00900, 154

P00901, 154

P00902, 154

P00904, 154, 155

P00905, 154, 155)

P00906, 154, 155

P00907, 1:32

P00908, 154, 156, 157 
P00909, 156, 157

P00910, 156, 157

P00911, 157

P00912, 156

PO00923, 147

P00924, 48, 66

P00925, 48, 66

P00927, 86

P00928, 158

P00929, 158

P00930, 158

P00931, 158

P00932, 158

P00933, 158

P00934, 152, 153

P00935, 97, 98, 100, 101

P00937, 154, 157

P00938, 52, 63

P00939, 52, 63

P00940, 52, 63

P00941, 52, 63

$\underline{\mathbf{P} 00942}, 52,63$

P00943, 52, 63

Poog6i3, 175

P00964, 58

P00965, 58

P00966, 8

P00967, 78, 79, 81, 121, 126, 127

P00968, 132, 133

P02721, 27, 35)

P02905, 106, 107

P03928, 27, 35

P03929, 27, 35 
P03930, 27, 35

P03931, 27, 35

P03932, 27, 35

P03933, 27, 35)

P03947, 15)

P():3945, !4

P03962, 136

P03963, 154

P03964, 157

P03965, 132

P04036, 9:3

P04046, 78, 12:3

$\underline{\mathbf{P} 04075}, 51,62$

P04076, 9

P04078, 5x

$\mathrm{P}(04079,45,131$

P04161, 79, 121

P04384, 22

P04391, 7, 8

P04394, 24

P04406, 50, 64

P04424, 9)

P045 36, $14 x$

P04763, 67

P04764, 45, 66

P04770, 5\%

P04771, 58

P04772, 58

P04773, 58

$\underline{\mathbf{P 0 4 7 8 9}}, 52,63$

P04790, 52, 53, 6.3

P04796, 50), 64

P04797, 50, 64 
P04828, 52, 63

P04947, 152

P04948, 152

P()49)(68, 86

P04970, 50, (54

P04990, 152

P05020, 134

P05021, 1355

P05022, 27, 35)

P05035, 136

P05036, 27, 28, 35, 36

P05037, 27, 35

P05038, 27, 28, 35, 36

P05039, 27, 35

P05040, 27, 35

P05042, 147, 148

P05062, 51, 62

P05063, 51, 62

P05064, 51, 62

P05065, 51, 62

P05081, 2, 128

$P(05082,2,128$

P05147, 12

P05148, 68

P05150, 7

P(0)5194, 12

P05195, 12

P05201, 176

P05202, 176

P05324, 70

P05325, 70

P05328, 154, 156, 157

P05370, 110 


\begin{tabular}{|c|}
\hline P05378, 154 \\
\hline P05379, 154 \\
\hline P05415, 5.5 \\
\hline P05416, 56 \\
\hline P05435, 27, 35 \\
\hline P05436, 27, 35 \\
\hline P05437, 27, 35) \\
\hline P05438, 27, 35) \\
\hline P05439, 27, 35 \\
\hline P05440, 27, 35 \\
\hline P05441, 27, 355 \\
\hline P05442, 27, 35) \\
\hline P05457, א \\
\hline P05466, 14 \\
\hline $\mathbf{P} 05492,27,35)$ \\
\hline P05493, 27, 35 \\
\hline P05494, 27, 35 \\
\hline P05495, 27, 35 \\
\hline P05496, 27, 35 \\
\hline P05498, 27, 35) \\
\hline P05499, 27, 35 \\
\hline P05500, 27, 355 \\
\hline P05504, 27, 35) \\
\hline P05626, 27, 35) \\
\hline P05630, 27, 35) \\
\hline $\mathbf{P} 05631,27,35$ \\
\hline P05632, 27, 35 \\
\hline P05640, 92 \\
\hline P05654, 1333 \\
\hline P05717, 27, 35 \\
\hline$P 05791,90,165,170$ \\
\hline$P 05792,86,87$ \\
\hline P(05793, 89, 164, 169 \\
\hline
\end{tabular}


P05796, 20

P05989, 89, 164, 169

P05990, 1:32, 13:3, 1:34

P06106, 20

P06168, \$9, 164, 169)

P06192, 17:3

P06201, 58

P06204, $1: 34$

P06283, 27, 28, 35, 36

P06284, 27, 28, 35, 36

P06285, 27, 35)

P06286, 27, 35

P06287, 27, 35

P06288, 27, 35

P06289, 27, 35

P06290, 27, 35)

P06291, 27, 35

P06450, 27, 35

P06451, 27, 35)

P06452, 27, 35)

P06453, 27, 35

P06528, 27, 35)

P06531, 154, 156, 157

P06540, 27, 35

P06541, 27, 35)

P06542, 27, 35

P06557, 154

P06558, 154

P06559, 155)

P06560, 156, 157

P06561, 158

P06562, 158

P06576, 27, 35 
P06633, 71

P06711, 58

P06721, 98, 101

P06733, 48, 66

P06744, 54, 60

P06745, 54, 60, 61

P06862, 140

P06958, 141

P06960, 7, 8

$\mathrm{P} 06977,50,64$

P06981, 44, 130

P06986, 72

P(069)7 7 71, 72

P06988, 73

P06989, 69, 70

P06994, 148, 149

P $96998,61,62$

P06999, 61, 62

P07001, 176, 177

P07002, 176, 177

P07004, 118, 119

P07005, 118

P07014, 31, 32, 145, 146

P07015, 144

P07022, 115, 116, 159)

P07023, 115, 159, 160

P07128, 152

P07137, 27, 35

P07138, 27, 35

P07170, 2, 128

P07172, 72

P07205, 49, 65)

P07227, 27, 35 
P07244, 78, 81, 126, 127

P07251, 27, 35)

P07258, 132

P07259, 132, 13:3

P07262, 57

P07285, 155)

P07322, 48, 66

P07323, 48, 66

P07341, 51, 62

P07342, 87, 162, 167

P07343, 147

P.07344, 158

P07345, 158

P07377, 49, 65

P07378, 49, 65

P07459, 144, 145

P07460, 144, 145

P07486, 50, 64

P07487, 50, 64

P07511, 173

P07513, 27, 35)

P07547, 11, 12, 1:3, 14

P07598, 180

P07600, 158

P07601, 158

P07603, 180

P07623, 97, 100

P07637, 14

P07638, 14

P07639, 11, 12

P07669, 52, 63

P07677, 27, 35

P07678, 27, 35 


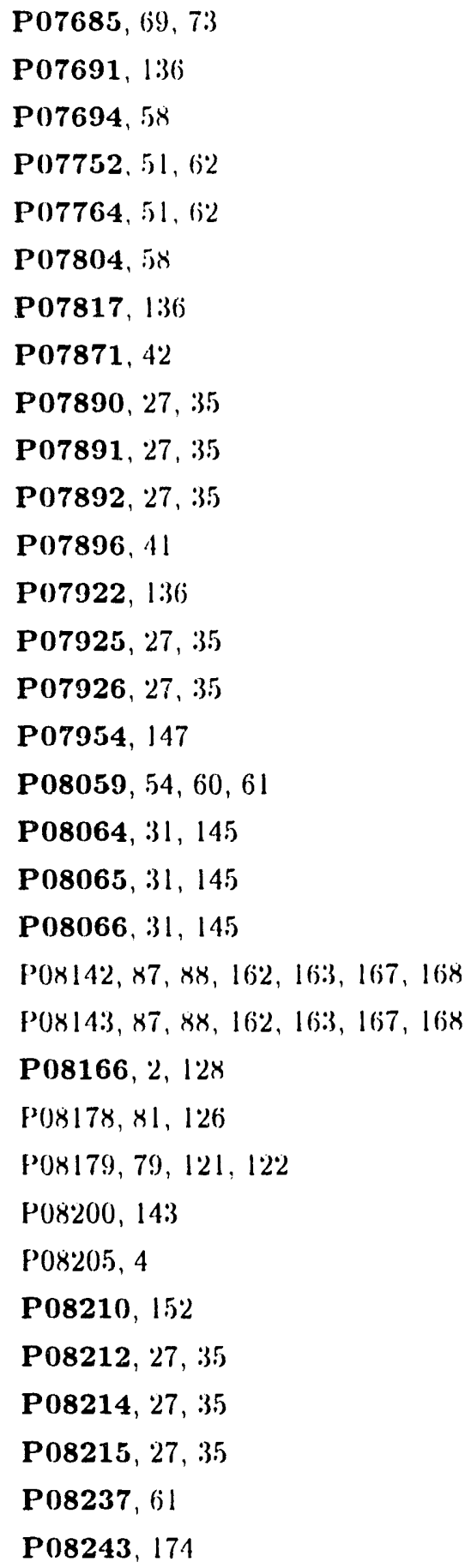


P08244, 136

P08249, 148

P08281, 58

P08282, 58

P08308, $T$

P08309, 135

P08:32:3, 56

P08:324, 48, 66

P08328, 139

P08:329, 13

P08:330, 77, 12:3

P08398, 1:37, 138

P08417, 147

P08420, 13:3

P08421, 133

P08428, 27, 35

P08439, 50, 64

P08440, 51, 62

P08444, 27, 35

P08445, 27, 35)

P08446, 27, 35

P08447, 27, 35)

P08448, 27, 35

P08449, 27, 35)

P08450, 27, 35)

P08477, 50, 64

P08495, 74

P08496, 74

P08499, 76

P08559, 141

P08566, 11, 12, 13, 14

P08660, 74, 75)

P08679, 142 
P08734, 4s, 66

P08735. 50, 64

P08870, 1:35

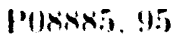

P08891 19, 45

P08892. 4!, 65)

P08893. 49, 65

P08906, 176

P089i17. 176

P08955. 1:32, 13:3. 1:34

P08966 4 4?, 65)

P08967. 4!. (65)

P0902x. $41, \times 2.121$

[09029, x1, x2. 12.1. 125)

Po9034. $x$

P09041, 49. 65)

P09043. 50. 64

P09044. 50. 64

P090:3. 170. 17]

P09060. 1.44

Po9061. 1.4

P00094. 50. 6.4

Po9104. Ax. 6iti

Po9110. 12

Pog114. אד. Hfi2. 16i

P09117. 51. ti2

P09123. 152

P09124. 50. 6i4

P09143. 144

P09188. 49. 6.5

P09195. 53)

P09199.

P099200, 33 
P09201, 53

P09202, 5.3

Po9218, 2T, 35

P09219, 27, 35

P09220, 25, 35)

P09221, 27, 35

P09222, 2 $\bar{\tau}, 35$

Po9315, 50, 64

P09316, 50, 64

P09317, 50, 64

P09329, 7T, 12:3

P09330, 7T, 12:3

Po9342, xí, 16i2, 16i

P09403, 49, 65

P09404, 49, 65)

P09411, 49, 65

P09457, 27, 35

P09463, 136

P09467. 53)

P09468, 27, 355

P09469, 2 27,35

P09556, 135, 1:36

P09575, 154, 157

P09606, 58

P09639, 27, 35

P09672, 50, 64

P09785, 154

P09786, 154

P09826. 5x

P098:31, 58, 59

P09X:32, 5x, 59

P09890, 95)

Po9948, 142 
P09972, 51, $6 \% 2$

P10096, 50, 64

P10097, 50, 64

P10173, 147

P10251, 2. 128

P10341. 116

P10366, 68

P10367, 69

P10368, 71, 72

P10369. 72

P10370. 73

Pl0:371, 70

P10372, 70

Plo444, 31, 32, 145, 14T

P10446, 31, 32, 145, 147

P10539, 75)

P10583, 58

P10584, 148

P10603, 27, 35

P10618, 50, 64

P10652, 1:36

P10656, 5x

P10658, $1: 39$

P10659, 22

P10719, 27, 35)

P10748, 14

P10772, 2, 128

P10801, 141

P10869, 74

P10880, 13

P10887, 14א

P10963, 47

P11019, 27, 35) 
P11024, 176

P11029, 106

P11043, 14

P11066, 7

P11080, 15x

P11081, 158

P11096, 20, 21

P11172, 135, 136

P11177, 141

P11243, 54, 60, 61

P11386, I4K

P11402, 27, 35)

P11410, 110

P11411, 110

P11412, 110

P11413, 110

P11445, 5

P11446, 5, 6

P11447, 9

P11497, 106

P115:37, 54, 60, 61

P11574, 27, 35

P11592, 27, 355

P11593, 27, 35)

P11600, 56

Pl1603, 50, 51, 64, 65.5

P11604, 51, 62, 6.3

P1 1608, 27, 35

P11665, 49, 65

P11708, $14 \%$

P11724, 7

P11725, 7

P11726, 7 


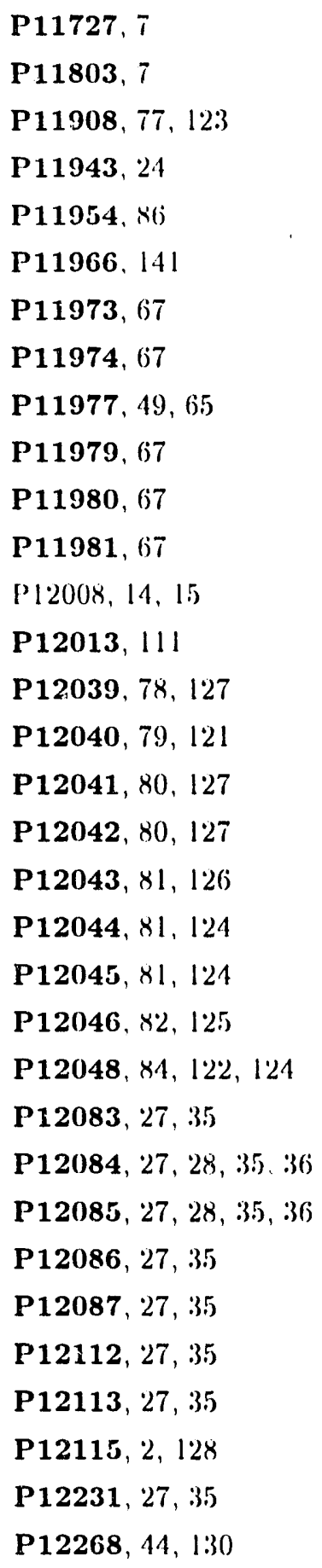


P12269, 44, 130

P12276, 107

P12283, 1. 129)

P12289, list

P12290, א א

P12291. L5N

P12298, 55)

P12299, 55

P12300, 55

P12320, 155

P12321, 155

P12341. 54. 60

P12343, I

P12344, 176

P12345, 176

P12382, 61

P12404, 27, 35

P12405, 27, 35)

P12406, 27, 35

P12407, 27, 35

P12408, 27, 35)

P12409, 2i , 35)

P12410, 27, 35)

P12421, 14

P12424, 5र

P12425, 5.

P12635, 180

P12636, I 80

P12646, 110

P12674, 20

P12696, 27, 35)

P12697, 27, 35

P12698, 2i, 2K, 35), 36 
P12699, 27,35

P12709, 54, 60,61

P12782, 49, 65)

P12783, 49, 65)

P12785, 107

P12858, 50, 64

P12859, 50, 64

P12860, 50, 64

P12862, 27, 35)

P12863, 52, 6.3

P12928, 67

P12943, 180

P12944, 180)

P12953, 27, 35

P12984, 27, 35)

P12985, 27, 35

P12986, 27, 35

P12987, 27, 35

P12988, 27, 35.

P12989, 27, 35

P12990, 27, 35)

P12991, 27, 35)

P13009, 99

P13048, 87, 89, 162, 1644, 167, 169

P13052, 27, 35)

P13061, I80

P13062, 180

P13063, 180)

P13064, I 10

P13065, 180

P13066, 180

P13221, 176

P13228, 15x 
P13242, 1:37

P13256, 8

P13257, 8

P13258. 132

P13298, 1355

P13356, 2T, 35)

P13357, 27, 35

P13375, 54, 60

P13376, 54, 60

P13377, 54, 60

P13437, 42

P13439, 135, 1:36

P13444, 22

P13499, 5 反

P13547, 27, 35

P13548, 27, 35)

P13564, 58

P13618, 27, 35)

P13619, 27, 35

P13620, 27, 35)

P13621, 27, 35

P13628, I 1 ()

P13629, Ir)

P13649, 136

P13663, 75

P13735, 47

P13929, 48, 66

P13997, 156

P14017, 136

P14062, 111

P14092, 27, 35)

P14093, 27, 35)

P14152, 14x 
P14165, 142

P14178, 67

P14193, 77, 12:3

P14223, 51, 62

P14228, 49, (65)

P14332, 111

P14383, 119

Pl4407, 147, 148

P14408, 147

P14413, 27, 35)

P14414, 27, 35

P14519, 173

P14540,51, 6i2

P14552, 158

P14568, 8

P14569, 27, 35)

P14570, 27, 35

P14571, 27, 35

P14572, 27, 35

P14604, 41

P14618, 67

P14636, 5.

P14637, 158

P14638, 158

P14654, 58

P14655, 58

P14656, 58

P14657, 57

P14671, 158

P14718, 156

P14766, 5:3

P14786, 67

P14828, 49, 65) 
P14843, 10

P14845, $1: 32$

P14846, 132

P14862, 27, 35

P14863, 27, 35)

P14874, 8T, 1622, 167

P14909, 176

P14926, 107, 108

P14952, 154

P14953, 154

P14964, 136

P14965, 136

P14995, 7

P15007, 4x, 66

P15012, 27, 35

P15013, 27, 35

P15014, 27, 35

P15015, 27, 35

P15044, 16

P15102, 58

P15103, 5x

P15104, 5x

P15105, 5x

P15106, 58

P15111, 57

P15115, 50, 64

P15124, 58

P15168, 90, 165

P15188, $1: 36$

P15254, 80, 127

P15280, 55

P1s283, 1 \%0

P15284, 180 
P15313, 27, 35

P15368, 107

P15395, 154

P15426, 52, 633

P15429, 4x, 66

P15474, 12

P15567, 81, 124

P15588, 110

P15623, 5

P156539, 84, 85, 122, 124

P15640,78, 79, 127

P15690, 24

P15770, 12, 13

P15849, 77, 12:3

P15994, 27, 35)

P15995, 27, 35)

P15996, 27, 35)

P15997, 27, 35)

P15998, 27, 35

P15999, 27, 35)

P16000, 27, 35)

P16001, 27, 35)

P16006, 51, 62

P161 ก), 14:3

P16120, 15:2

P16140, 27, 35)

P16142, $14 x$

P16245, 73

P16246, 72

P16247, 71

P16250, 7)

P16280, 14

P16304, 2, 128 


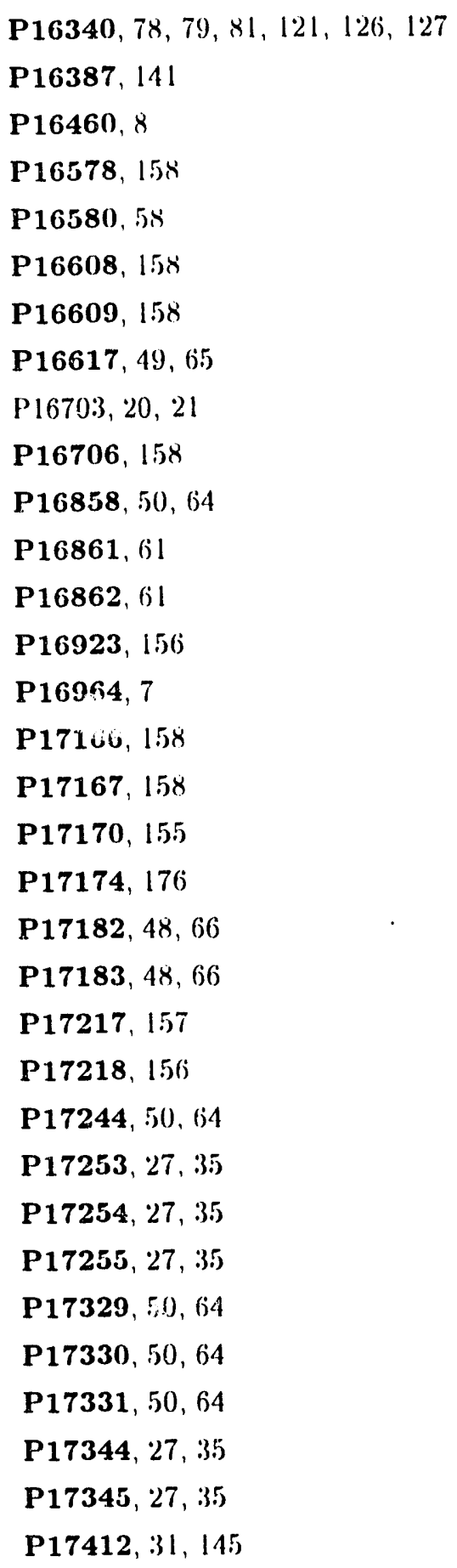


P1741.3, 31, 145

P17423, 152

P17505, $14 \mathrm{~K}$

P17562. 22

P17596. 31. 145

P17597, $8 T, 16 \%, 167$

P17604, 27, 35

P17605, 27, 35

P17614, 27, 35

P17632, 180

P17633, I 80

P17674, 27, 35)

P17675, 27, 35

P17688, 14

P17694, 24

P17714, 174

P17721, 50, 64

P17729, 50, 64

P17730, 50, 64

P17731, 72

P17736, 72

P17751, 52, 633

P17783, 148

P17784, 51, 6i2

P17812, $1: 37$

P17817, 119

P17819, 50, 64

P17856, II

P17857, I1

P17858, 61

P17878, 50, 64

P17902, 139)

P18132, 135) 
P18133, 179

P18185, 1:32

P18186, 7

P18187, 1к()

P18188, 180)

P18190, Is0

P18191, 180

P18240, 54, 60

P18260, 27, 35

P18261, 155)

P18267, 154

P18268, 157

P18284, 158

P18285, 158

P18298, 22

P18304, 157

P18335, 6

P18483, 154, 156, 157

P18544, 6

P18636, 180

P18637, 180)

P18786, 7:3

P18787, 71

P18789, 142

P18819, 57

P18859, 27, 35)

P18904, 135

P18912, 49, 65

P18949, 98, 101

P19023, 27, 35

P19064, 58

P19080, 115, 159

P19089, 50, 64 


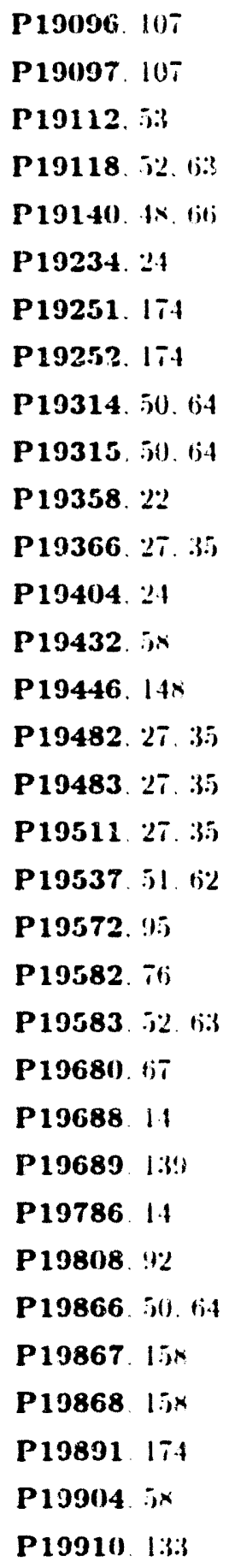




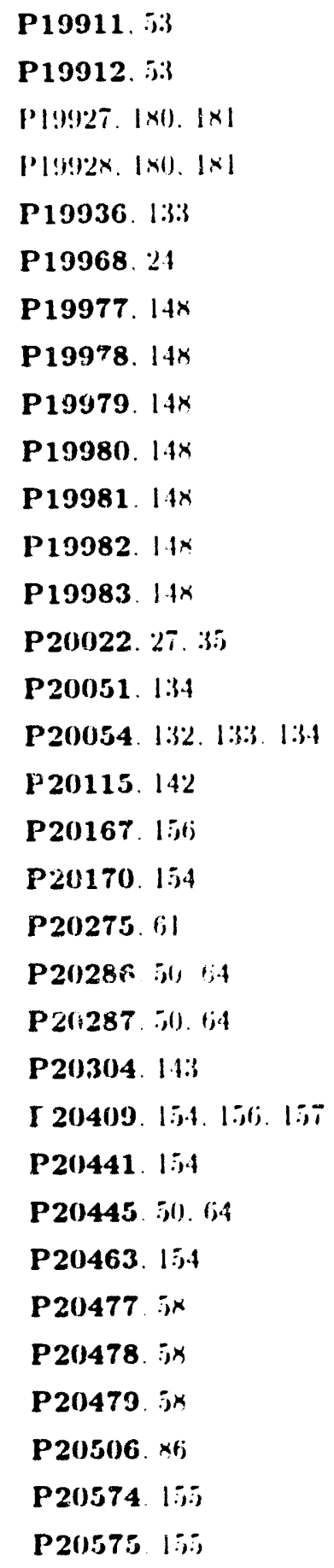


P20576, 154

P20577, 157

P20578, 157

P20579, 154

P20580, 154

P20597. 132

P20599, 2 7,35$)$

P20600, 27, 35

P20601, 27, 35)

P20602, 27, 35

P20603, 27, 35)

P20673. 4)

P20691. 14

P20692. 160)

P20707, 144

P20772, $7 x, x 1,126,127$

P20805, 5x

P20839, 44, $1: 30$

P20858, 27, 28, 35, 36

P20859. 27, 35)

P20901. 1.42

P20902. 142

P20003. 142

P20921, 31, 14:

P20922. 31. 145

P20967, 144

P20971, 49, 65)

P20972, 49, 65)

P21108, 77, 12:3

P.21151, 42

P21154. 5x

P.21155, X2, X3, 125. 126

P.21177. 41. 12 


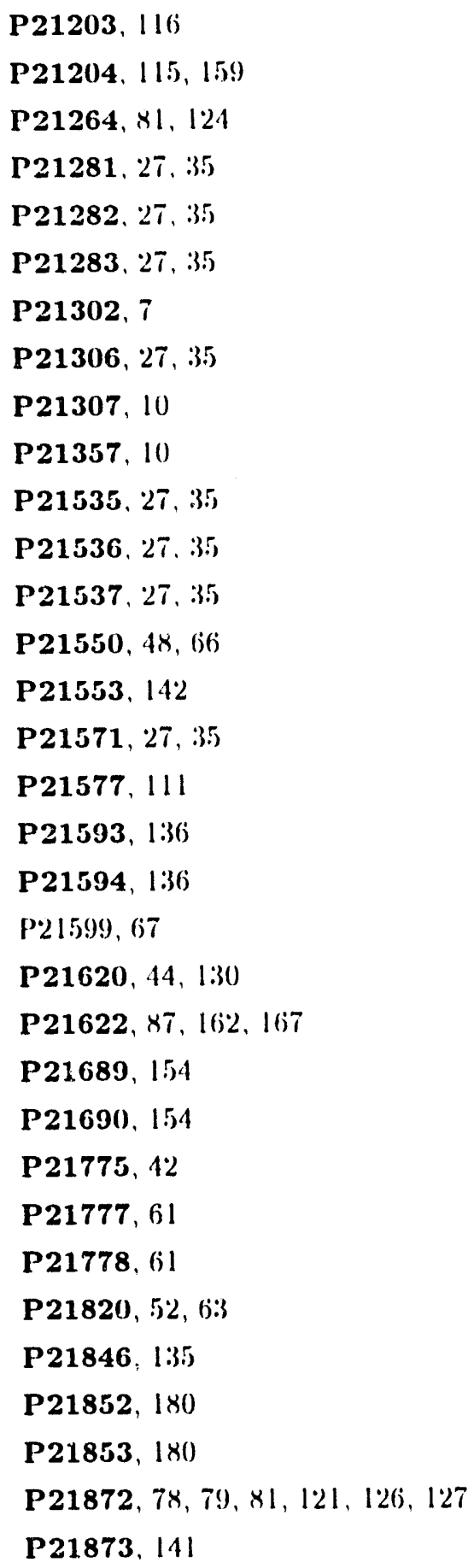




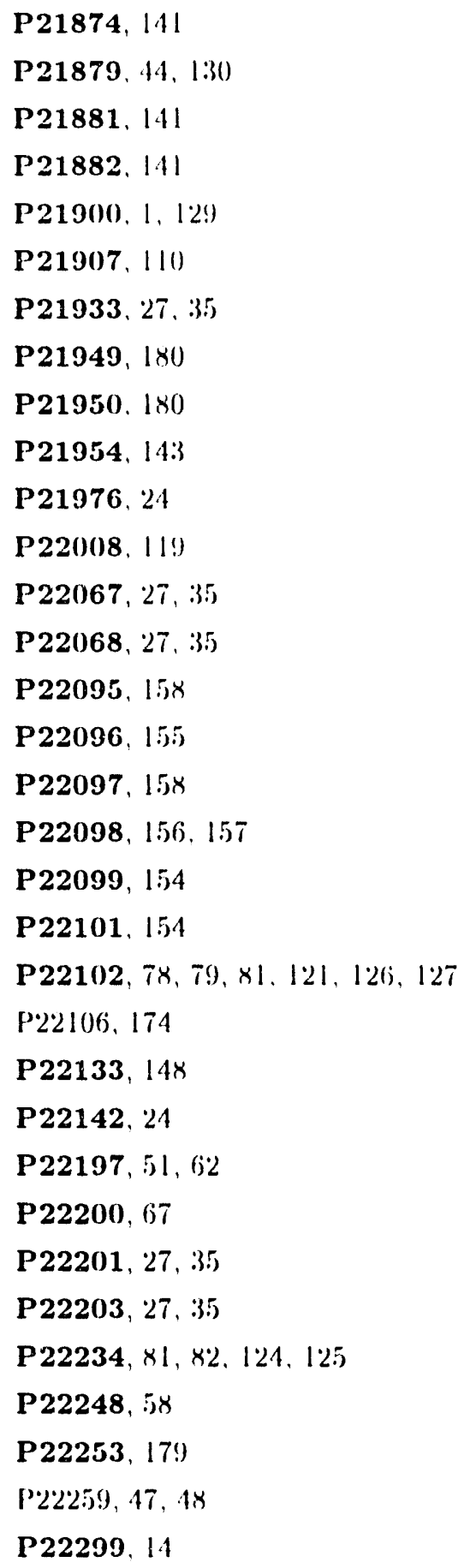




$$
\begin{aligned}
& \text { P22350, 119) } \\
& \text { P22360, } 67 \\
& \text { P22418, 5:3 } \\
& \text { P22476, 27, 35) } \\
& \text { P22477, 27, 35) } \\
& \text { P22478, 2T, 35 } \\
& \text { P22479, 27, } 35 \\
& \text { P22480, 27, 35) } \\
& \text { P22481, 27, 35) } \\
& \text { P22482, 27, 35) } \\
& \text { P22483, 27, 35) } \\
& \text { P22487, I } \\
& \text { P22512,50, 6i } \\
& \text { P22513, 50, 64 } \\
& \text { P22550, 27, 35) } \\
& \text { P22567, } 4 \\
& \text { P22572, 1:32 } \\
& \text { P22662, 2 } 7,35) \\
& \text { P22663, 27, 35) } \\
& \text { P22675, 9 } \\
& \text { P22721, 27, 35) } \\
& \text { P22722, 27, 35) } \\
& \text { P'2767, } x, 9 \\
& \text { P22768, } x \\
& \text { P22778, 27, 35) } \\
& \text { P22780, 5.3 } \\
& \text { P22878, א } \\
& \text { P.22992, 110, } 111 \\
& \text { P23007, } 142 \\
& \text { P23014, 5.3 } \\
& \text { P23034, } 176 \\
& \text { P23039, 27, 35) } \\
& \text { P23040, 27, 35 }
\end{aligned}
$$




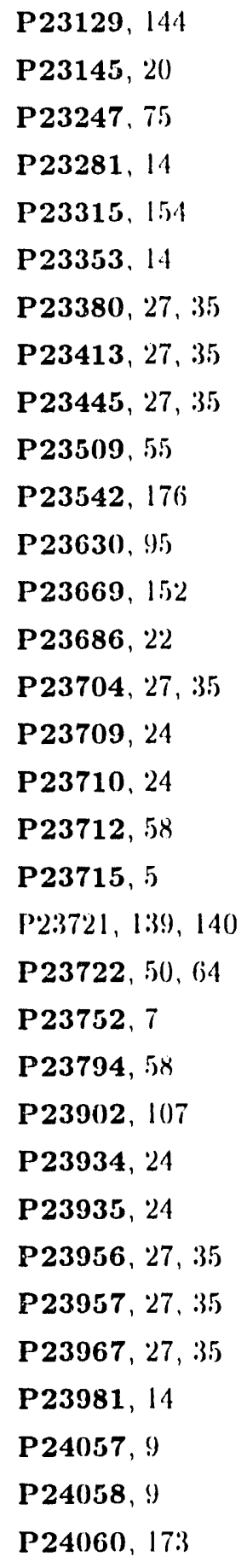




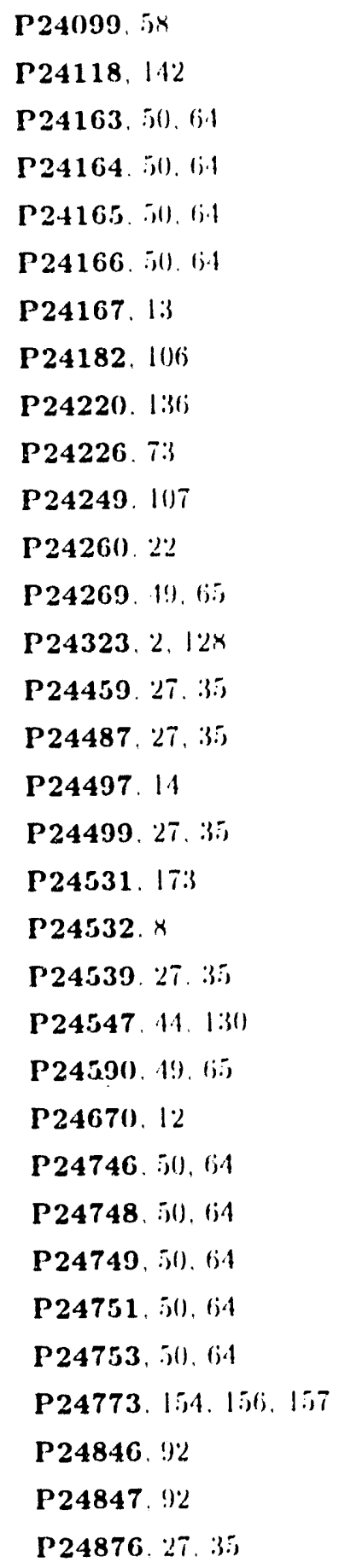


P24888, 2T, 35)

P24917, 24

P24918, 24

P24919, 24

P24920, 156, 157

P24945, 2T, 35)

P24946, 27, 35

P24947, 27, 35)

P24948, 27, 35)

P24949, 27, 35)

P25004, 27, 35)

P25005, 27, 35)

P25055, 49, 65)

P25075, 27, 35)

P25077, 148

P25126, 144

P25163, 2T, 35)

P25164, 27, 35)

P25170, 154, 156, 157

P25269, 158

P25284, 24

P25306, 86

P25379, 86

P25462, 58

P25468, 135

P25515, 27, 35)

P25665, 99)

P25696, 48, 66

P25704, 48, 66

P25705, 27, 35)

P25708, 24

P25710, 24

P25711, 24 


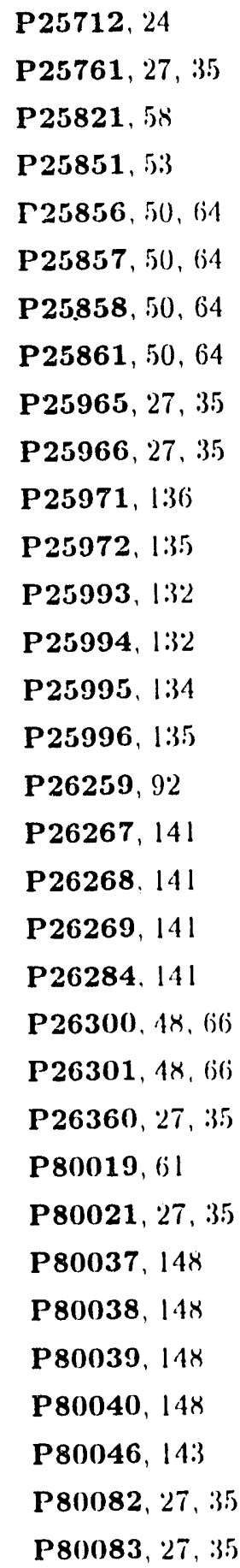


P80084, 27, 3

P80085, 27, 35)

P80086, 27, 35

P80087, 27, 35)

P80088, 27, 35

\section{Reactions}

1,3-bisP'glycerate_1, 65)

1,3-bisP'glycerate_2, 50

1-(o-carboxyphenylamino)-1'-deoxy ribulose-5)-p'TRP'-1, 157

1-keto-2-MethylValerate_ILE_1, 90

10-Formyl-TIF_THF_1, 151

2-aceto-2-Hydroxy-Butyrate_ILE_L, 89

2-aceto-Lactate-VAL_1, 164, 169)

2-keto-3-Methyl-Valerate_JLE_1, 90

2-keto-Isovalerate_VAL_1, 1655

2-keto-Isovalerate_VAL_2, 170

2-Ketobutyric_acid_ILE_1, 87

2-ket.oglutarate_l, 114

2 -Ketoghutarale $2,5 x$

$2-P^{2}$-glycrerate-1, 66

2-P-glycerate_2, 4x

3-(teoxy-D)-A rabino-Heptulosonate-7-P'-1, 11

3-Enolpyruvyl-Shikimat.r-5P-1, 14

3-Hydroxy Acyl-(ioA_-1, 41

3-KetoAcyl-('oA_l, 42

3-P-glycerate_-1, 66

3-P-glycerate_2, 49

3-P-HydroxyPyruvate_SER_1, 1:39

3-P-Serine_SER_1, 140

30)-Butyryl-A('P_Fat, I, I0X 
4'-p-n1-pantothenoylcysteine_( 'oA_1, 17

4 -p-panthetheine_('OA_L, 17

4'-p-pantothenate_('od_1, 16

5 -p-rihosyl-4-succinocarloxamid(n-5)-Al_Purine-1, 8.3

5)-P-ribosyl-5-AI_P'urine-1, s'

5)-dehydro-Quinate-1, 12

5-dehydro-Shikimate_1, 12

5)-P-ribosyl-4-('arboxy-5-Al_Purine_1, 82

5-P-Ribosyl-5-Formamido-4-Imid-( arboxamide_Purine_1, 84

5-P'-Ribosyl-Amine_P'urine_1, is

5-P-Ribosyl-(ilycineanide_P'urine-1, 79

(i-P-gluconate-1, 111

6-P-gluconolactone-1, 111

AcetoAcetyl-S'-A('P'Fat__1, 108

acetyl ( o. $-1,142$

Acetyl-('oA_Fat_l, 106

Acetyl-CoA_Fat_2, 106

Acetyl-(iLUL_1, 5

Acyl-( 'oA_1, 40

Adenylosucc_Adenine-1, 1

ADP-glucose_1, 56

ADP_1, 26, 34

ADP_Adenine_1, 3

AlC:AR_P'urine_1, 84

Alanine-Synthesis_1, 172

alpha,beta-Dihydroxy-Isovalerate_VAL_1, 165, 170

AMP_Adenine_1, 2

Anthranilate_TRP_1, 155

Argininosucc_l 1,9

ARci_creatine_1, 19

ATP-Synthesis_1, 128

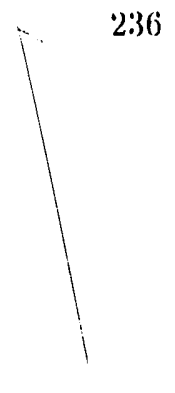


Sirhamoyl-L-Aspartate_Pyrimidine_1, 134

('arbamoyl-P'Pyrimidine_1, 13:3

('horismate_1, 115), 159)

('horismate_TRP_1, 154

(itrate-1. 142

('itrulline_l, $x$

('omplexll_1, 31

('omplexl.1, 24

('reatine_('reatine_1, 172

('reatine_('reatine_2, 172

('rotonyl-A('P_Fat_1, 109)

( yt-15,5,56-2e-1, 25, 333

(:yt-1.55566_1, 25, 33

( $y(t-1) 55)$ - $-2 e-1,25,33$

( $y\left(t-1,5,5 x_{-} 1,25,33\right.$

('yt-D-2e_1, 25, 34

( yt-O-2e_1, 26, 34

D-Erythro-Lrnidazole-(ilycerol-P_HIS_L, 71

Deamiclo-NAD_NAD_1, 105)

Dephospho-('oA_CoA-1, is

[Di-H1-Orotate_f'yrimidine_1, 13:5

Dihydrodipicolinate_1, 93

dihydroxyacet.one-P_1, 52, 6.3

Enoyl-('oA_1, 41

fatty acid_l, 40

F(iAM_Purine_1, 81

F(iAR_Purine_1, 80

FMN_FAD_1, 39

fructose-1, (b-bisP_1, 53.3

fructose-1,6-bisp'-2, 622

fructose-(j-P-1, 61 
fructose- $\left(6-P^{2}-2,54\right.$

fumarate_1, 147

(:3P_SER L 1 139)

(iD)P_(inanosille-1, 16

(iLT_L, 5

(iLT_2, IIS

(iLT_3, 4

glucose-1,6-bisP-1, 5)

glucose-1-P-2, 5;

glucose-6-P_1, 110

glucose- $\left(j-P_{-}-3,60\right)$

glucose-(6-P_-4, 5.)

glucose_1, 60, 110

(ilutamate-Synthesis_2, i)

(ilutamine_Pyrimidine_1, 132

glyceraldehyde-3-P_-1, 64

glyceraldehycle-3-P_3, 51

glyceraldehyde-3-P-P.4, 5:2

(ilycine-Synthesis_1, 17:3

(:LY_(iLY-1, 17:3

(iMP_(iuanosine-1, 45)

('TP-Synthesis_1, 130

Histidinol_HIS_L, 73

Homo-('YS'L, 99

Homo-('YSL2, 102

Homo-('YS_C:YS_1, 2:3

Homo-SER_1, 97, 100

Homo-SER_, 152

Imidazole-Acetol-P_HIS_L, 7 I

IMP-Syuthesis_1, 121

IMP_Adenime-1, I 
IMI'_(inanosine-1 4.4

Indole-3-cilycerol-P.TRP-1. 15i

isucit rate-1. 113

L. Asparagiun-Syuthesis-1. 179

L. A-paragine-Synthesis.2. 175

L-Asparagine-Synthesis_3. 175

L-Aspartate-Sernialdehyide_-1,92

L-Aspart ate-Semialdehyde_2. i6

L-Aspartate-Synthesis_1, 176

L-Aspartate-1, it

L-beta-Aspartyl-P-1, is

L-( ystathionime-1, 9x. 10)

L-( yistathionine_('YS_1, 2:3 .

|-alelta(1)-pyroline_j-carboxylate_1, 119

L-Lamma-(ilutamyl-Phosphate-1, Ilx

L-filut amate gamma-Semialdehyde_L, 119

L-Histidinol-P_HIS_L. T2

L-(O)rnithine $1, i$

L-()rnithine_( reatine_1. 19

L.L-Dhaminopimelate-1. 95

malate_l. 1.4k

Malonyl-AC'P_Fat_I, I0T

Malonyl-e 'od_Fat_L I0T

meso-Diaminopimelate-1, 95

Methenyl-Tetrahydrofolate_TIIF_1, 1.50

Methylene-Tetrahydrofolate_THF_L, 150

MET_rYS.L. 22

n-(5)-phosphoribosyl)-anthranilate_TRP_L, 156

X-Acetyl-(ilutamyl-P'L, ;

X-Acetylilutamate_bemaldehyde_l, 6

X-Alphit-Acetyl-()rnithine_L $T$ 
X-suce-keto-1-Amimopimelate_1.94

n-succinylll-2,(6-diaminopimelate_1, 94

NADPHE-1. 176

NADP.NAD 1.17

NAI).NAI) IT

NAD_NAD_L ITK

VAD_NAD_3. ITX

Niacinamide_YAD_L, 10:3

Viacinamide_NAD_2. 179

Niacin_NAD_1. 179

Virctinamide_Yucleoticle_NAD_L 103

Virotinamide_Vucleotide_.NAD_2, 180

Nicotinamide_Ribose_.XAD_L I I ()

Vicotinate_Nucleotide_NAD_1, 104

()-Acetyl-L-Serine_( YS-1. 20

()-Phospho-L-HomoSerine_1, 152

()-Surrinyl-L-Homoserine_1, 97, 100

(Orotate_Pyrimidine_1. 135)

orotidine-5is-p.Pyrimidine_1. 1:36

isxalacetate-Ecoli_l. it

P-enolpyrunate_1. 67

P-enolpyruvate_2, 4x

P-hydroxy-PhenylPyruvate-TYR_l, 160

Pantothenate_('od_L If

PEP-1, 10

Phenyl-Pyruvate_1, 116

Phosphoribosyl-AMP_IIIS_1, 699

Phosphoribosyl-ATP_HIS_I, 69

Phosphoribosyl_Forminino-5)-A1-4-( arboxarnide_Ribotide_IHIS_I, i0

Phosphoribulosyl_Formimino-i)-Al-4-('arboxamide_Ribotide_IIIS_1, 7 I

P'rephenate-1, 116

Prephenate_TYR_1. 161) 
PRPP_HIS_L, 68

PRPP_Purine - 1, is

pyruvate_2, 141

pyruvate_3, 47

Pyruvate_VAL_1, 162, 167

Quinolinate_NAD_1, 104

Ribotlavin_FAD_1, 39)

Rihose-5P'Purine-1, it

ribuluse-5-P-1, 112

ribulose-5-P-2, 112

sedoheptulose-7-P_1, 114

SER_('YSSL1, 20)

Shikimate-5P-1, 14

Shikimate_1, 13

succinate_-1, 145)

succinyl (oA_l, 144

Tetrahydrodipicolinate-1, 9:3

THR_ILE_L, 86

Bhiguinone_1, 180

1/DP_Pyrimidine_1, $1: 37$

UMP_Pyrimidine_L, 137

U'TP_Pyrimidine-1, 1:37

Xanthosine-5)-P'(inanosine-1, 45)

xylulose-5-P-1, 11:3

xyluiose-5-P.2, 11:3 

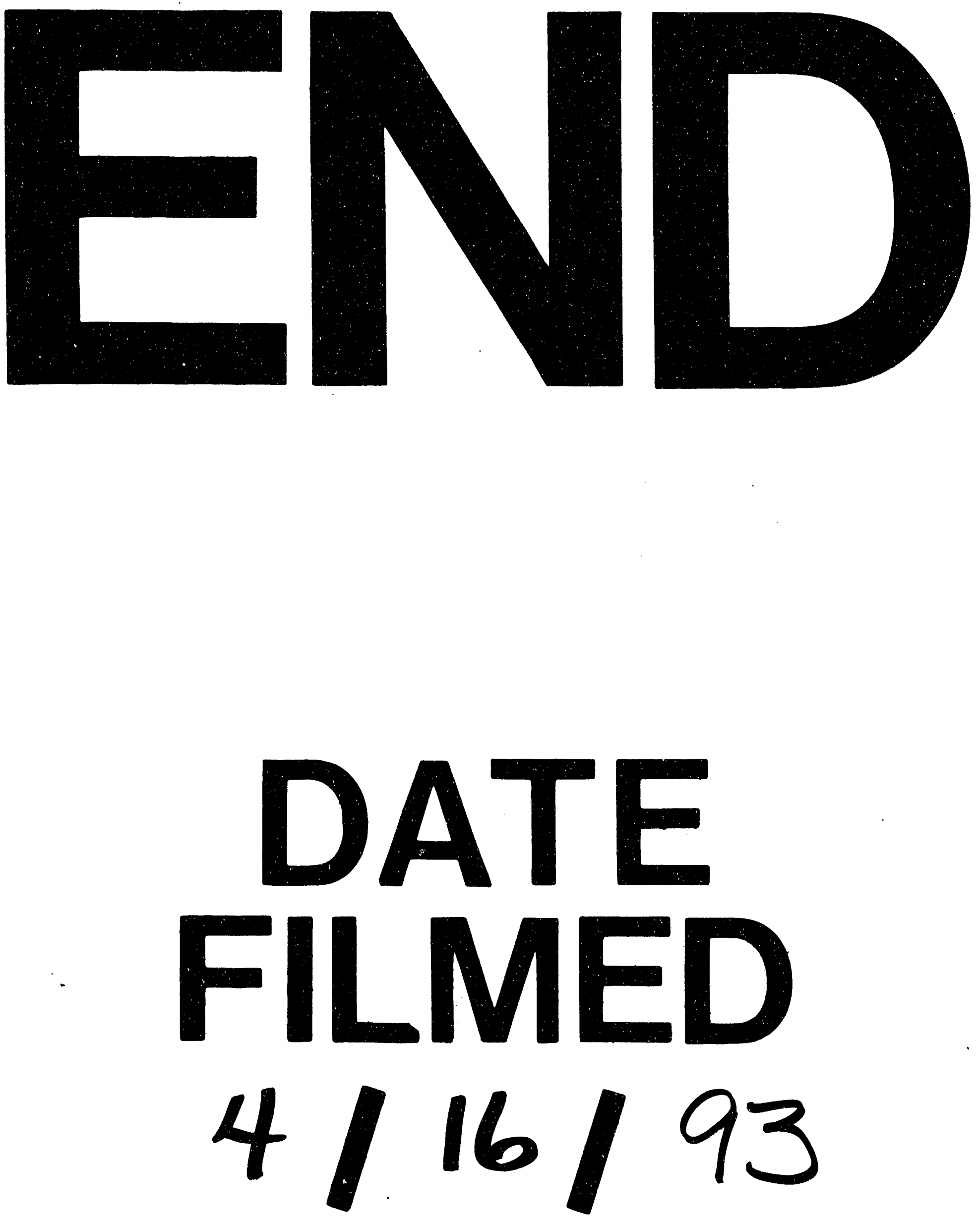

1 
